\title{
Realização de Campos Livres de Álgebras de Kac-Moody Afim
}

\author{
Marcela Guerrini Alves
}

\section{DissERTAÇÃO APRESENTADA}

$\mathrm{AO}$

Instituto DE Matemática e Estatística

DA

UNIVERSIDADE DE SÃO PAULO

PARA

OBTENÇÃO DO TÍTULO

DE

Mestre em Matemática

Área de Concentração: Álgebra

Orientador: Prof. Dr. Vyacheslav Futorny

Durante o desenvolvimento deste trabalho a autora recebeu auxílio financeiro do CNPq 


\section{Realização de Campos Livres de Álgebras de Kac-Moody Afim}

Esta versão da dissertação contém as correções e alterações sugeridas pela Comissão Julgadora durante a defesa da versão original do trabalho, realizada em 08/08/2016. Uma cópia da versão original está disponível no

Instituto de Matemática e Estatística da Universidade de São Paulo.

Comissão Julgadora:

- Prof. Dr. Vyacheslav Futorny (orientador) - IME-USP

- Prof. Dr. Raul Antonio Ferraz - IME-USP

- Prof. Dr. Renato Alessandro Martins - UNIFESP 


\section{Agradecimentos}

À minha mãe Elisa, por tudo o que aprendi através do exemplo.

À primeira professora de Matemática que me ensinou o encanto das possibilidades, Elisabete Teresinha Guerato. Minha gratidão também a todos os outros professores que fizeram e ainda fazem parte da minha história, fundamentais para o meu desenvolvimento.

Ao Rômulo, meu marido, por ter acreditado e respeitado os caminhos que decidi trilhar, mesmo nos momentos de maior dificuldade, em que isso tenha afetado significativamente nossa vida.

Aos meus amigos, dentro e fora da Universidade, importantíssimos nesse intrincado quebracabeça que é a existência humana.

Tenho imensa gratidão à vida, por todas as oportunidades que me foram dadas, e estudar Matemática foi uma delas, sem dúvida uma das mais transformadoras.

Agradeço ao CNPq pelo auxílio financeiro. 


\section{Resumo}

AlVES, M. G. Realização de Campos Livres de Álgebras de Kac-Moody Afim. 2016. Dissertação de Mestrado - Instituto de Matemática e Estatística, Universidade de São Paulo, São Paulo, 2016.

Este trabalho tem como objetivo principal estudar módulos irredutíveis sobre as álgebras de Kac-Moody afim, conforme [7].

Em particular, a técnica de localização foi aplicada aos módulos de Verma imaginários sobre a álgebra de Lie afim $A_{1}^{(1)}$, com o objetivo de obter novos módulos irredutíveis sobre essa álgebra. Conforme [8] e [6], é o mesmo que aplicar a técnica de localização à primeira realização de campos livres de $A_{1}^{(1)}$.

Para cumprir o objetivo, introduzimos as álgebras de Kac-Moody, tendo como foco principal as álgebras de Kac-Moody do tipo afim, conforme [14]. Em seguida, definimos os módulos de Verma, destacando os módulos de Verma imaginários sobre a álgebra de Lie afim $A_{1}^{(1)}$, conforme [8].

Palavras-chave: álgebras de Kac-Moody afim, módulos de Verma, localização, realização de campos livres. 


\section{Abstract}

ALVES, M. G. Free Fields Realization of Affine Kac-Moody Algebras. 2016. Dissertação de Mestrado - Instituto de Matemática e Estatística, Universidade de São Paulo, São Paulo, 2016.

The main purpose of this work is to study the irreducible modules of affine Kac-Moody algebras, according to [7].

In particular, the localization technique was applied to the imaginary Verma modules of affine Lie algebra $A_{1}^{(1)}$, with the purpose to obtain new irreducible modules of this algebra. According to [8] and [6], it is the same as to apply the localization technique to the first realization of free fields of $A_{1}^{(1)}$.

To achieve the purpose, we introduced the Kac-Moody algebras, having the main focus the affine Kac-Moody algebras, according to [14]. Following, we defined the Verma modules, highlighting imaginary Verma modules of affine Lie algebra $A_{1}^{(1)}$, according to [8].

Keywords: affine Kac-Moody algebras, Verma modules, localization, free fields realization. 


\section{Sumário}

$\begin{array}{ll}\text { Lista de Símbolos } & \text { ix }\end{array}$

Introdução

1 Álgebras de Kac-Moody 1

1.1 Definições e Construções Iniciais $\ldots \ldots \ldots$. . . . . . . . . . . . . . . . 1

1.1.1 Realização de Matrizes . . . . . . . . . . . . . . . . . . . . . . . . . . . 1

1.1.2 Construção da Álgebra de Lie Auxiliar . . . . . . . . . . . . . . . . . . . . 2

1.1.3 Construção da Álgebra de Kac-Moody . . . . . . . . . . . . . . . . . . . . . 4

1.2 A Forma Bilinear Invariante . . . . . . . . . . . . . . . . . . . . . 5

1.3 Representações Integráveis e o Grupo de Weyl . . . . . . . . . . . . . . . . . . . . . . 8

1.4 Uma Classificação das Matrizes de Cartan Generalizadas . . . . . . . . . . . . . . . . . 13

1.5 Raízes Reais e Raízes Imaginárias . . . . . . . . . . . . . . . . . . . . . . . . . 19

1.6 Álgebras de Kac-Moody Afim . . . . . . . . . . . . . . . . . . . . . 22

2 Módulos de Verma $\quad 31$

2.1 Produto Tensorial . . . . . . . . . . . . . . . . . . . . . . . 31

2.2 Decomposição Triangular . . . . . . . . . . . . . . . . . . . . 35

2.3 Módulos de Verma . . . . . . . . . . . . . . . . . . . . . . . . . . 40

$2.3 .1 \quad$ A Categoria $\mathcal{O} \ldots \ldots \ldots \ldots \ldots \ldots \ldots$

2.4 Módulos de Verma Imaginários _ . . . . . . . . . . . . . . . . . . . . . . 44

2.4.1 Módulos de Verma Imaginários sobre a Álgebra de Lie Afim $A_{1}^{(1)} \ldots \ldots$. . . . 47

2.5 Módulos de Verma J-Imaginários . . . . . . . . . . . . . . . . . . . . . . . . 52

2.5.1 Realização de Campos Livres de $\hat{\mathfrak{s} r}(2, \mathbb{C}) \ldots \ldots \ldots$. . . . . . . . . 54

3 Realização de Campos Livres $\quad 59$

3.1 Caso de Dimensão Finita . . . . . . . . . . . . . . . . . . . . . . . . . . 59 
3.2 Caso Afim . . . . . . . . . . . . . . . . . . . . . . . . 61

3.2 .1 Segunda Realização de Campos Livres . . . . . . . . . . . . . . . . . . 65

4 Localização de Álgebras não Comutativas $\quad 79$

4.1 Anéis Comutativos . . . . . . . . . . . . . . . . . . . . . 79

4.1.1 Módulos sobre Anéis Comutativos . . . . . . . . . . . . . . . . . 82

4.2 Anéis não Comutativos . . . . . . . . . . . . . . . . . . . 83

4.2.1 Módulos sobre Anéis não Comutativos . . . . . . . . . . . . . . . . . . . 91

4.3 Um Caso Particular . . . . . . . . . . . . . . . . . . . . . . . . . 102

5 Localização da Primeira Realização de Campos Livres 105

5.1 Notação e Convenções . . . . . . . . . . . . . . . . . . . . . . . . . . . 105

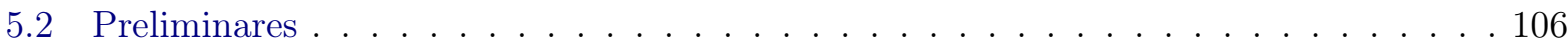

5.2 .1 Módulos de Verma Imaginários . . . . . . . . . . . . . . . . . . . . . . . . 106

5.2 .2 Primeira Realização de Campos Livres . . . . . . . . . . . . . . . . . . . 106

5.3 Irredutibilidade de Módulos Localizados . . . . . . . . . . . . . . . . . . . . 109

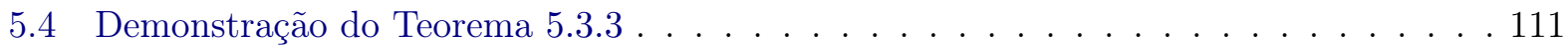

5.4 .1 Vetores Primitivos no Caso $J-i K \in \mathbb{Z} \ldots \ldots \ldots \ldots \ldots \ldots$

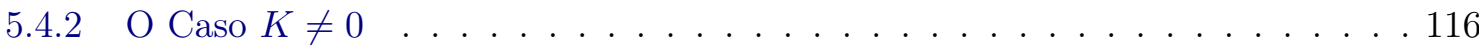

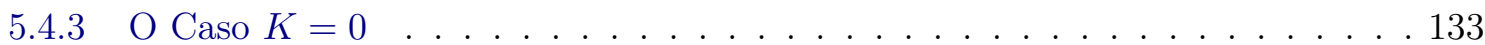

A Notação, Definições e Resultados Básicos 137

A.1 Anéis e Módulos . . . . . . . . . . . . . . . . . . . . . . 137

A.2 Álgebras de Lie . . . . . . . . . . . . . . . . . . . . . . . 140

A.2.1 Representações de Álgebras de Lie . . . . . . . . . . . . . . . . . . . . . . . . 145

A.3 Álgebras Envolventes Universais . . . . . . . . . . . . . . . . . . . . . . . . 152

A.4 Extensões Centrais . . . . . . . . . . . . . . . . . . . . . 154

$\begin{array}{ll}\text { Referências Bibliográficas } & 157\end{array}$ 


\title{
Lista de Símbolos
}

\author{
$A^{t} \quad$ a matriz transposta da matriz $A$ \\ $\mathbb{N} \quad$ o conjunto dos números inteiros estritamente positivos \\ $\mathbb{Z} \quad$ o conjunto dos números inteiros \\ $\mathbb{Z}_{+} \quad$ o conjunto dos números inteiros não negativos \\ $\mathbb{Q} \quad$ o corpo dos números racionais \\ $\mathbb{R} \quad$ o corpo dos números reais \\ $\mathbb{C} \quad$ o corpo dos números complexos \\ $\mathbb{K} \quad$ o corpo $\mathbb{Q}, \mathbb{R}$ ou $\mathbb{C}$ \\ $V^{*} \quad$ o espaço dual de um espaço vetorial $V$ \\ $\langle.,$.$\rangle \quad o pareamento entre um espaço vetorial e seu dual$
}




\section{Introdução}

O objetivo principal desta dissertação é estudar uma nova família de módulos sobre a álgebra de Kac-Moody afim $\hat{\mathfrak{s l}}(2, \mathbb{C})$, obtidos a partir da primeira realização de campos livres, bem como os critérios de irredutibilidade desses novos módulos, seguindo um dos resultados obtidos por Vyacheslav Futorny, Dimitar Grantcharov e Renato A. Martins em [7], no ano de 2014.

Descreveremos a seguir a forma como o trabalho será organizado, e o conteúdo em cada capítulo.

Em primeiro lugar, destacamos a importância de apresentar definições e resultados básicos sobre as álgebras de Lie e suas representações. Isso será feito no apêndice A, que divide-se da seguinte forma: na primeira seção, enunciaremos alguns conceitos necessários para a construção da teoria de localização em anéis (e álgebras) não comutativos, tendo em vista sua utilização no Capítulo 4. Em seguida, teremos o conteúdo referente às álgebras de Lie propriamente ditas: álgebras de Lie e suas representações, álgebras envolventes universais e extensões centrais. Ao longo do apêndice, faremos referência à bibliografia onde cada resultado pode ser encontrado sem maior dificuldade.

O Capítulo 1 tem por objetivo introduzir as álgebras de Kac-Moody, seguindo exclusivamente [14]. Ao longo das seções, passaremos pelos resultados importantes sobre essas álgebras, e utilizaremos as matrizes de Cartan generalizadas para que possamos classificá-las em tipos: finito, afim e indeterminado. Após a classificação, estudaremos especificamente as do tipo afim, grupo ao qual pertence o objeto de estudo deste trabalho, a álgebra de Kac-Moody afim $\hat{\mathfrak{s l}}(2, \mathbb{C})$, que é uma álgebra de Lie de dimensão infinita.

No Capítulo 2, o principal objeto de estudo serão os módulos de Verma imaginários sobre as álgebras de Kac-Moody afim, sua caracterização e critérios de irredutiblilidade. O capítulo divide-se em seções nas quais definiremos decomposição triangular de uma álgebra de Lie, módulos de Verma padrão sobre uma álgebra de Lie com decomposição triangular, módulos de Verma imaginários sobre álgebras de Lie afim, enunciando os resultados apresentados por Futorny em [8], e em seguida os módulos de Verma imaginários sobre $\hat{\mathfrak{s l}}(2, \mathbb{C})$, seguindo [9]. Ao final da seção, apresentaremos brevemente os módulos de Verma J-imaginários, estudados por Renato A.Martins em [16]. 
Na sequência, o Capítulo 3 destina-se a apresentar ao leitor o conceito de realização de campos livres de álgebras de Kac-Moody afim. Em particular, para a álgebra $\hat{\mathfrak{s} l}(2, \mathbb{C})$, explicitaremos a primeira realização através de exemplos. Como complemento à teoria, optamos por fornecer ao leitor uma breve exposição da segunda realização, também para a álgebra objeto de nosso estudo.

No Capítulo 4 construiremos o anel de frações (ou álgebra de frações) para uma álgebra associativa, com unidade, não necessariamente comutativa. Tal construção nos é interessante para aplicação à álgebra envolvente universal de $\hat{\mathfrak{s} l}(2, \mathbb{C})$, tendo em vista que ao demonstrarmos os critérios de irredutibilidade dos novos módulos que encontraremos necessitaremos "mergulhar"a álgebra envolvente em outra álgebra, onde certos elementos particulares possuam inversos.

Por fim, o Capítulo 5 destina-se ao objetivo principal deste trabalho. Partindo da primeira realização de campos livres, e fixando um número inteiro, serão exibidos os novos módulos e será provado o teorema que explicita os critérios de irredutibilidade dos mesmos, e a técnica de localização será aplicada exatamente nessa demonstração. O capítulo será dividido em seções e subseções de modo a desenvolver a demonstração dos lemas, proposições e corolários que nos fornecem a demonstração desejada. 


\section{Capítulo 1}

\section{Álgebras de Kac-Moody}

Nosso principal objetivo neste capítulo é estudar a construção das álgebras de Kac-Moody afim "não torcidas", o que será feito através de duas abordagens. Na primeira delas, estudaremos a construção a partir das matrizes de Cartan generalizadas; na segunda, como uma extensão de álgebras de Kac-Moody do tipo finito. A notação, bem como a maioria das construções deste capítulo, seguem ([14]). Destacamos que algumas informações são também encontradas em detalhes em $([4])$.

\subsection{Definições e Construções Iniciais}

\subsubsection{Realização de Matrizes}

Definição 1.1.1. Seja $A=\left(a_{i j}\right)_{i, j=1}^{n}$ uma matriz complexa $n \times n$. A é uma matriz de Cartan generalizada se, para todo $i, j=1, \ldots, n$, satisfaz as seguintes condições:

(a) $a_{i i}=2$;

(b) $a_{i j}$ são inteiros não positivos, se $i \neq j$;

(c) $a_{i j}=0$ implica $a_{j i}=0$.

Definição 1.1.2. Seja $A=\left(a_{i j}\right)_{i, j=1}^{n}$ uma matriz complexa $n \times n$ de posto l. Uma realização de $A$ é uma tripla $\left(\mathfrak{h}, \Pi, \Pi^{V}\right)$, em que $\mathfrak{h}$ é um espaço vetorial sobre $\mathbb{C}, \Pi=\left\{\alpha_{1}, \ldots, \alpha_{n}\right\} \subset \mathfrak{h}^{*} e$ $\Pi^{V}=\left\{\alpha_{1}^{V}, \ldots, \alpha_{n}^{V}\right\} \subset \mathfrak{h}$ são subconjuntos indexados em $\mathfrak{h}^{*}$ e $\mathfrak{h}$, respectivamente, satisfazendo as seguintes condições:

(a) ambos os conjuntos $\Pi e \Pi^{V}$ são linearmente independentes;

(b) $\left\langle\alpha_{i}^{V}, \alpha_{j}\right\rangle=a_{i j},(i, j=1, \ldots, n)$;

(c) $n-l=\operatorname{dim} \mathfrak{h}-n$.

Definição 1.1.3. Seja $A=\left(a_{i j}\right)_{i, j=1}^{n}$ uma matriz complexa $n \times n$ de posto l. Sejam $\left(\mathfrak{h}, \Pi, \Pi^{V}\right) e$ $\left(\mathfrak{h}_{1}, \Pi_{1}, \Pi_{1}^{V}\right)$ duas realizações de $A$, em que $\Pi=\left\{\alpha_{1}, \ldots, \alpha_{n}\right\}, \Pi^{V}=\left\{\alpha_{1}^{V}, \ldots, \alpha_{n}^{V}\right\}, \Pi_{1}=\left\{\beta_{1}, \ldots, \beta_{n}\right\}$ e $\Pi_{1}^{V}=\left\{\beta_{1}^{V}, \ldots, \beta_{n}^{V}\right\}$. Diremos que essas realizações são isomorfas se existe um isomorfismo de espaços vetoriais $\phi: \mathfrak{h} \longrightarrow \mathfrak{h}_{1}$ tal que $\phi\left(\alpha_{i}^{V}\right)=\beta_{i}^{V}$ e $\phi^{*}\left(\beta_{i}\right)=\alpha_{i}$, em que $\phi^{*}$ é a transformação linear (isomorfismo) transposta de $\phi$. 
Proposição 1.1.4. Seja $A=\left(a_{i j}\right)_{i, j=1}^{n}$ uma matriz complexa $n \times n$. Então, a menos de isomorfismo, existe uma única realização de $A$.

(vide $[14, \mathrm{p} .2]$ ).

Observação 1.1.5. Seja $A=\left(a_{i j}\right)_{i, j=1}^{n}$ uma matriz complexa $n \times n$. Se $\left(\mathfrak{h}, \Pi, \Pi^{V}\right)$ é uma realização de $A$, então $\left(\mathfrak{h}^{*}, \Pi^{V}, \Pi\right)$ é uma realização de $A^{t}$.

Definição 1.1.6. Dadas duas matrizes complexas $A_{1}$ e $A_{2}$, e suas realizações $\left(\mathfrak{h}_{1}, \Pi_{1}, \Pi_{1}^{V}\right)$ e $\left(\mathfrak{h}_{2}, \Pi_{2}, \Pi_{2}^{V}\right)$, podemos obter uma realização da soma direta $\left(\begin{array}{cc}A_{1} & 0 \\ 0 & A_{2}\end{array}\right)$ dessas matrizes:

$$
\left(\mathfrak{h}_{1} \oplus \mathfrak{h}_{2}, \Pi_{1} \times\{0\} \cup\{0\} \times \Pi_{2}, \Pi_{1}^{V} \times\{0\} \cup\{0\} \times \Pi_{2}^{V}\right),
$$

denominada soma direta das realizações.

Definição 1.1.7. Uma matriz complexa A (e sua correspondente realização) é denominada decomponivel se, após reordenar os índices (isto é, efetuar uma permutação de suas linhas e a mesma permutação das colunas), A decompõe-se em uma soma direta não trivial.

Destacamos a seguir alguma notação e nomenclatura que serão utilizadas com frequência ao longo do capítulo:

- Para os conjuntos da Definição 1.1.2, usamos a seguinte terminologia: $\Pi$ é denominado base de raízes, $\Pi^{V}$ base de co-raízes, e seus elementos são respectivamente as raízes simples e co-raízes simples.

- Definimos também:

$$
Q=\sum_{i=1}^{n} \mathbb{Z} \alpha_{i} \text { e } Q_{+}=\sum_{i=1}^{n} \mathbb{Z}_{+} \alpha_{i}
$$

$Q$ é denominado reticulado de raízes.

- Para $\alpha=\sum_{i=1}^{n} k_{i} \alpha_{i} \in Q$, o número $h t \alpha:=\sum_{i=1}^{n} k_{i}$ é a altura de $\alpha$.

- Introduzimos uma ordem parcial $\geq$ em $\mathfrak{h}^{*}$ definindo $\lambda \geq \mu$ se $\lambda-\mu \in Q_{+}$.

\subsubsection{Construção da Álgebra de Lie Auxiliar}

Nesta seção daremos os primeiros passos na construção de uma álgebra de Kac-Moody a partir de uma matriz de Cartan generalizada.

Seja $A=\left(a_{i j}\right)_{i, j=1}^{n}$ uma matriz $n \times n$ sobre $\mathbb{C}$, e seja $\left(\mathfrak{h}, \Pi, \Pi^{V}\right)$ uma realização de $A$. Em primeiro lugar, introduzimos uma álgebra de Lie auxiliar $\widetilde{\mathfrak{g}}(A)$ com geradores $e_{i}, f_{i}(i=1, \ldots, n)$ e $\mathfrak{h}$, e definida pelas seguintes relações:

$$
\left\{\begin{aligned}
{\left[e_{i}, f_{j}\right] } & =\delta_{i j} \alpha_{i}^{V} & (i, j=1, \ldots, n), \\
{\left[h, h^{\prime}\right] } & =0 & \left(h, h^{\prime} \in \mathfrak{h}\right), \\
{\left[h, e_{i}\right] } & =\left\langle\alpha_{i}, h\right\rangle e_{i} & \\
{\left[h, f_{i}\right] } & =-\left\langle\alpha_{i}, h\right\rangle f_{i} & (i=1, \ldots, n ; h \in \mathfrak{h}) .
\end{aligned}\right.
$$


Pela unicidade da realização de $A$ (Proposição 1.1.4), $\mathfrak{\mathfrak { g }}(A)$ depende apenas de $A$.

Denotando por $\widetilde{\mathfrak{n}}_{+}$(respectivamente $\left.\widetilde{\mathfrak{n}}_{-}\right)$a subálgebra de $\widetilde{\mathfrak{g}}(A)$ gerada por $e_{1}, \ldots, e_{n}$ (respectivamente $\left.f_{1}, \ldots, f_{n}\right)$, temos o seguinte resultado:

Teorema 1.1.8. (a) $\widetilde{\mathfrak{g}}(A)=\widetilde{\mathfrak{n}}_{-} \oplus \mathfrak{h} \oplus \widetilde{\mathfrak{n}}_{+}$(soma direta de espaços vetoriais).

(b) $\widetilde{\mathfrak{n}}_{+}$(respectivamente $\tilde{\mathfrak{n}}_{-}$) é livremente gerada por $e_{1}, \ldots, e_{n}$ (respectivamente $f_{1}, \ldots, f_{n}$ ).

(c) A aplicaçãa $e_{i} \longmapsto-f_{i}, f_{i} \longmapsto-e_{i}(i=1, \ldots, n), h \longmapsto-h(h \in \mathfrak{h})$, pode ser unicamente estendida a uma involução $\widetilde{\omega}$ da álgebra de Lie $\widetilde{\mathfrak{g}}(A)$.

(d) Em relação a h, existe uma decomposição em espaços de raízes:

$$
\widetilde{\mathfrak{g}}(A)=\left(\bigoplus_{\substack{\alpha \in Q_{+} \\ \alpha \neq 0}} \tilde{\mathfrak{g}}_{-\alpha}\right) \oplus \mathfrak{h} \oplus\left(\bigoplus_{\substack{\alpha \in Q_{+} \\ \alpha \neq 0}} \widetilde{\mathfrak{g}}_{\alpha}\right),
$$

em que $\tilde{\mathfrak{g}}_{\alpha}=\{x \in \tilde{\mathfrak{g}}(A) \mid[h, x]=\alpha(h) x$ para todo $h \in \mathfrak{h}\}$. Além disso, dim $\tilde{\mathfrak{g}}_{\alpha}<\infty$, e $\widetilde{\mathfrak{g}}_{\alpha} \subset \widetilde{\mathfrak{n}}_{ \pm}$, para $\pm \alpha \in Q_{+}, \alpha \neq 0$.

(e) Dentre os ideais de $\widetilde{\mathfrak{g}}(A)$ intersectando $\mathfrak{h}$ trivialmente, existe um único ideal maximal $\tau$. Além disso,

$$
\tau=\left(\tau \cap \widetilde{\mathfrak{n}}_{-}\right) \oplus\left(\tau \cap \widetilde{\mathbf{n}}_{+}\right) \text {(soma direta de ideais). }
$$

(vide [14, p.4-5]).

Observação 1.1.9. Por definição, $\widetilde{\mathfrak{n}}_{+}$é gerada por monômios de Lie em $e_{1}, \ldots, e_{n}$, e $\widetilde{\mathfrak{n}}_{-}$por monômios de Lie em $f_{1}, \ldots, f_{n}$.

Da demonstração do item (d) do teorema anterior,

$$
\widetilde{\mathfrak{n}}_{+}=\bigoplus_{\substack{\alpha \in Q_{+} \\ \alpha \neq 0}} \widetilde{\mathfrak{g}}_{\alpha}, \widetilde{\mathfrak{n}}_{-}=\bigoplus_{\substack{\alpha \in Q_{+} \\ \alpha \neq 0}} \widetilde{\mathfrak{g}}_{-\alpha} \text { e } \mathfrak{h}=\widetilde{\mathfrak{g}}_{0}
$$

o que implica $\widetilde{\mathfrak{g}}_{\alpha} \subset \widetilde{\mathfrak{n}}_{+}$e $\widetilde{\mathfrak{g}}_{-\alpha} \subset \widetilde{\mathfrak{n}}_{-}$, para todo $\alpha \in Q_{+}, \alpha \neq 0$.

Portanto, $\widetilde{\mathfrak{g}}_{\alpha}$ e $\widetilde{\mathfrak{g}}_{-\alpha}$ são gerados por monômios de Lie em $e_{1}, \ldots, e_{n}$ e $f_{1}, \ldots, f_{n}$, respectivamente, e podemos descrever esses monômios.

Seja $\alpha \in Q_{+}, \alpha \neq 0, \alpha=\sum_{i=1}^{n} k_{i} \alpha_{i}$. Usando as relações (1.1) e a identidade de Jacobi, prova-se que um monômio em $e_{1}, \ldots, e_{n}$ é um elemento de $\widetilde{\mathfrak{g}}_{\alpha}$ se e somente se $e_{i}$ "aparece"exatamente $k_{i}$ vezes para cada $i$; e um monômio em $f_{1}, \ldots, f_{n}$ é um elemento de $\widetilde{\mathfrak{g}}_{-\alpha}$ se e somente se $f_{i}$ "aparece"exatamente $k_{i}$ vezes, para cada $i$.

Portanto, para todo $\alpha \in Q_{+}, \alpha \neq 0$,

$$
\begin{aligned}
\tilde{\mathfrak{g}}_{\alpha} & =\sum_{\alpha_{i_{1}}+\ldots+\alpha_{i_{s}}=\alpha} \mathbb{C}\left[\ldots\left[\left[e_{i_{1}}, e_{i_{2}}\right], e_{i_{3}}\right] \ldots e_{i_{s}}\right] \\
\tilde{\mathfrak{g}}_{-\alpha} & =\sum_{\alpha_{i_{1}}+\ldots+\alpha_{i_{s}}=\alpha} \mathbb{C}\left[\ldots\left[\left[f_{i_{1}}, f_{i_{2}}\right], f_{i_{3}}\right] \ldots f_{i_{s}}\right] .
\end{aligned}
$$


Em particular, $\widetilde{\mathfrak{g}}_{\alpha_{i}}=\mathbb{C} e_{i}, \widetilde{\mathfrak{g}}_{-\alpha_{i}}=\mathbb{C} f_{i}$ e $\widetilde{\mathfrak{g}}_{s \alpha}=\{0\}$, se $|s|>1$.

Utilizando a identidade de Jacobi, temos ainda que $\left[\widetilde{\mathfrak{g}}_{\alpha}, \widetilde{\mathfrak{g}}_{\beta}\right] \subset \widetilde{\mathfrak{g}}_{\alpha+\beta}$, para todo $\alpha, \beta \in Q$.

\subsubsection{Construção da Álgebra de Kac-Moody}

Sejam $A$ uma matriz complexa $n \times n,\left(\mathfrak{h}, \Pi, \Pi^{V}\right)$ uma realização de $A$, e $\widetilde{\mathfrak{g}}(A)$ a álgebra de Lie com geradores $e_{i}, f_{i}(i=1, \ldots, n)$ e $\mathfrak{h}$, definida pelas relações (1.1). Pelo Teorema 1.1.8 (a), a aplicação natural $\mathfrak{h} \longrightarrow \widetilde{\mathfrak{g}}(A)$ é injetiva. Seja $\tau$ o ideal maximal nas condições de (e). Definimos então a álgebra de Lie

$$
\mathfrak{g}(A)=\widetilde{\mathfrak{g}}(A) / \tau
$$

Cabem aqui algumas considerações importantes, sintetizando o que já vimos:

- A matriz $A$ é denominada matriz de Cartan da álgebra de Lie $\mathfrak{g}(A)$, e $n$ é o posto de $\mathfrak{g}(A)$.

- A álgebra de Lie $\mathfrak{g}(A)$ cuja matriz de Cartan é uma matriz de Cartan generalizada é denominada álgebra de Kac-Moody.

- Será mantida a mesma notação para as imagens de $e_{i}, f_{i}(i=1, \ldots, n), \mathfrak{h}$ em $\mathfrak{g}(A)$. Portanto, salvo menção em contrário, ao utilizar essa notação estaremos nos referindo aos elementos em $\mathfrak{g}(A)$.

- A subálgebra $\mathfrak{h}$ de $\mathfrak{g}(A)$ é denominada subálgebra de Cartan, e os elementos $e_{i}, f_{i}$, com $i=1, \ldots, n$, são os geradores de Chevalley. Como $\tau$ intersecta $\mathfrak{h}$ trivialmente, a subálgebra de Cartan $\mathfrak{h}$ em $\mathfrak{g}(A)$ tem dimensão $2 n-l$, em que $l$ é o posto de $A$.

A partir da estrutura de $\widetilde{\mathfrak{g}}(A)$ vista no Teorema 1.1.8, podemos obter alguns detalhes a respeito da estrutura de $\mathfrak{g}(A)$, que listamos abaixo.

Segue de (1.2) que existe a seguinte decomposição em espaços de raízes com respeito a h:

$$
\mathfrak{g}(A)=\bigoplus_{\alpha \in Q} \mathfrak{g}_{\alpha}
$$

em que $\mathfrak{g}_{\alpha}=\{x \in \mathfrak{g}(A) \mid[h, x]=\alpha(h) x$, para todo $h \in \mathfrak{h}\}$ é o espaço de raízes ligado a $\alpha$, com $\mathfrak{g}_{\alpha} \cong \widetilde{\mathfrak{g}}_{\alpha} / \tau_{\alpha}, \tau_{\alpha}=\tau \cap \widetilde{\mathfrak{g}}_{\alpha}$. Em particular, $\mathfrak{g}_{0} \cong \mathfrak{h}$, pois $\tau$ intersecta $\mathfrak{h}$ trivialmente .(estamos fazendo uso da Proposição 1.5 de ([14, p.8-9]), que nos fornece $\left.\tau=\bigoplus_{\alpha \in Q} \tau \cap \widetilde{\mathfrak{g}}_{\alpha}\right)$.

O número mult $\alpha:=\operatorname{dim}_{\mathfrak{g}_{\alpha}}$ é a multiplicidade de $\alpha$. Como $\mathfrak{g}_{\alpha} \cong \widetilde{\mathfrak{g}}_{\alpha} / \tau_{\alpha}$, mult $\alpha<\infty$, para todo $\alpha \in Q$.

Um elemento $\alpha \in Q$ é denominado raiz se $\alpha \neq 0$ e mult $\alpha \neq 0$. Uma raiz $\alpha>0$ (respectivamente $\alpha<0)$ é denominada positiva (respectivamente negativa). Segue de (1.2) que cada raiz é ou positiva ou negativa. Denotaremos por $\Delta, \Delta_{+}$e $\Delta_{-}$os conjuntos de todas as raízes, raízes positivas e raízes negativas respectivamente. Então,

$$
\Delta=\Delta_{+} \cup \Delta_{-} \text {(união disjunta) }
$$

Notemos que se $\alpha>0$, então $\mathfrak{g}_{\alpha}$ é gerado por elementos da forma $\left[\ldots\left[\left[e_{i_{1}}, e_{i_{2}}\right], e_{i_{3}}\right] \ldots e_{i_{s}}\right]$; e se $\alpha<0, \mathfrak{g}_{\alpha}$ é gerado por elementos da forma $\left[\ldots\left[\left[f_{i_{1}}, f_{i_{2}}\right], f_{i_{3}}\right] \ldots f_{i_{s}}\right]$, tais que $\alpha_{i_{1}}+\ldots+\alpha_{i_{s}}=\alpha$ no 
primeiro caso, e $-\alpha$ no segundo. Em particular,

$$
\mathfrak{g}_{\alpha_{i}}=\mathbb{C} e_{i}, \mathfrak{g}_{-\alpha_{i}}=\mathbb{C} f_{i}, \mathfrak{g}_{s \alpha_{i}}=\{0\}, \text { se }|s|>1,(i=1, \ldots, n)
$$

Observamos ainda que $e_{i} \neq 0$ em $\mathfrak{g}(A)$, pois se $e_{i} \in \tau$, então $\alpha_{i}^{V}=\left[e_{i}, f_{i}\right] \in \tau \cap \mathfrak{h}$, o que contradiz $\mathfrak{h} \cap \tau=\{0\} ;$ de modo análogo, $f_{i} \neq 0$ em $\mathfrak{g}(A)$.

Seja $\mathfrak{n}_{+}$(respectivamente $\mathfrak{n}_{-}$) a subálgebra de $\mathfrak{g}(A)$ gerada por $e_{1}, \ldots, e_{n}$ (respectivamente $\left.f_{1}, \ldots, f_{n}\right)$. Então,

$$
\mathfrak{n}_{+}=\bigoplus_{\alpha>0} \mathfrak{g}_{\alpha} \cong \widetilde{\mathfrak{n}}_{+} / \tau \cap \widetilde{\mathfrak{n}}_{+}, \mathfrak{n}_{-}=\bigoplus_{\alpha<0} \mathfrak{g}_{\alpha} \cong \widetilde{\mathfrak{n}}_{-} / \tau \cap \widetilde{\mathfrak{n}}_{-}
$$

e temos a decomposição triangular

$$
\mathfrak{g}(A)=\mathfrak{n}_{-} \oplus \mathfrak{h} \oplus \mathfrak{n}_{+} \text {(soma direta de espaços vetoriais) }
$$

A involução $\widetilde{\omega}$ induz um automorfismo involutivo $\omega$ na álgebra de Lie $\mathfrak{g}(A)$, denominado involução de Chevalley, determinado por

$$
\begin{aligned}
& \omega\left(e_{i}\right)=-f_{i}, \omega\left(f_{i}\right)=-e_{i},(i=1, \ldots, n) \\
& \omega(h)=-h, \text { se } h \in \mathfrak{h} .
\end{aligned}
$$

Como $\tau$ é um ideal maximal de $\widetilde{\mathfrak{g}}(A)$, e $\widetilde{\omega}$ é um automorfismo involutivo, temos que $\widetilde{\omega}(\tau)$ é um ideal maximal de $\widetilde{\mathfrak{g}}(A)$. Como $\widetilde{\omega}(\tau) \subset \widetilde{\mathfrak{n}}_{-} \oplus \widetilde{\mathfrak{n}}_{+}$, então intersecta $\mathfrak{h}$ trivialmente, de onde concluímos que $\widetilde{\omega}(\tau)=\tau$. Assim, $\widetilde{\omega}$ induz um automorfismo involutivo

$$
\begin{aligned}
\omega: \mathfrak{g}(A) & \longrightarrow \mathfrak{g}(A) \\
\bar{x} & \longmapsto \overline{\widetilde{\omega}(x)}
\end{aligned}
$$

com as propriedades desejadas. Como uma consequência imediata temos que $\omega\left(\mathfrak{g}_{\alpha}\right)=\mathfrak{g}_{-\alpha}$, o que nos dá $m u l t \alpha=$ mult $(-\alpha)$. Em particular, $\Delta_{-}=-\Delta_{+}$.

Proposição 1.1.10. O centro da álgebra de Lie $\mathfrak{g}(A)$ é igual a

$$
\mathfrak{c}:=\left\{h \in \mathfrak{h} \mid\left\langle\alpha_{i}, h\right\rangle=0, \forall i=1, \ldots, n\right\}
$$

Além disso, $\operatorname{dim} \mathfrak{c}=n-l$.

(vide $[14, \mathrm{p} .11])$.

\subsection{A Forma Bilinear Invariante}

Nesta seção, nosso objetivo é a obtenção de uma forma simétrica bilinear não degenerada (e invariante) em $\mathfrak{g}(A)$. Para tanto, iniciamos com a definição de uma forma simétrica bilinear na subálgebra $\mathfrak{h}$ de $\mathfrak{g}(A)$.

Definição 1.2.1. Seja $A=\left(a_{i j}\right)$ uma matriz $n \times n$. Diremos que $A$ é simetrizável se existe uma 
matriz diagonal inversível $D=\operatorname{diag}\left(\epsilon_{1}, \ldots, \epsilon_{n}\right)$ e uma matriz simétrica $B=\left(b_{i j}\right)$ tais que

$$
A=D B
$$

A matriz $B$ é então denominada simetrização de $A$ e $\mathfrak{g}(A)$ é denominada álgebra de Lie simetrizável.

Sejam agora $A$ uma matriz simetrizável com uma decomposição (1.8) fixada e (h, $\left.\Pi, \Pi^{V}\right)$ uma realização de $A$. Fixando um subespaço $\mathfrak{h}^{\prime \prime}$ complementar a $\mathfrak{h}^{\prime}=\sum \mathbb{C} \alpha_{i}^{V}$ em $\mathfrak{h}$, definimos uma forma simétrica bilinear a valores em $\mathbb{C}(. \mid$.) em $\mathfrak{h}$ pelas seguintes equações:

$$
\begin{aligned}
& \left(\alpha_{i}^{V} \mid h\right)=\left\langle\alpha_{i}, h\right\rangle \epsilon_{i}, \forall h \in \mathfrak{h}, i=1, \ldots, n \\
& \left(h^{\prime} \mid h^{\prime \prime}\right)=0, \forall h^{\prime}, h^{\prime \prime} \in \mathfrak{h}^{\prime \prime} .
\end{aligned}
$$

Por (1.8) e aplicando (1.9), temos

$$
\left(\alpha_{i}^{V} \mid \alpha_{j}^{V}\right)=\left\langle\alpha_{i}, \alpha_{j}^{V}\right\rangle \epsilon_{i}=a_{j i} \epsilon_{i}=b_{i j} \epsilon_{i} \epsilon_{j}=\left\langle\alpha_{j}, \alpha_{i}^{V}\right\rangle \epsilon_{j}=\left(\alpha_{j}^{V} \mid \alpha_{i}^{V}\right)(i, j=1, \ldots, n) .
$$

Como $\alpha_{i}^{V}, \ldots, \alpha_{n}^{V}$ são linearmente independentes, então (. | .) está bem definida.

Lema 1.2.2. (a) O kernel da restrição da forma bilinear (. . .) a $\mathfrak{h}^{\prime}$ coincide com $\mathfrak{c}$.

(b) A forma bilinear (.| .) é não degenerada em h.

Demonstração. (a) Por definição, o kernel da restrição da forma bilinear (. | .) a h' é o conjunto

$$
\left\{y \in \mathfrak{h} \mid\left(\alpha_{i}^{V} \mid y\right)=0, \forall i=1, \ldots, n\right\}
$$

que é o próprio $\mathfrak{c}$, conforme Proposição 1.1.10

(b) Suponhamos que exista um elemento $\sum_{i=1}^{n} c_{i} \alpha_{i}^{V}$ tal que para todo $h \in \mathfrak{h}$

$$
0=\left(\sum_{i=1}^{n} c_{i} \alpha_{i}^{V} \mid h\right)=\left\langle\sum_{i=1}^{n} c_{i} \epsilon_{i} \alpha_{i}, h\right\rangle
$$

Então, $\sum_{i=1}^{n} c_{i} \epsilon_{i} \alpha_{i}=0$, pois o pareamento entre um espaço vetorial e seu dual é uma forma bilinear não degenerada. Portanto, $c_{i}=0(i=1, \ldots, n)$, já que $\alpha_{1}, \ldots, \alpha_{n}$ são linearmente independentes e $\epsilon_{i} \neq 0, \forall i$.

Tendo em vista que a forma bilinear (. | .) é não degenerada, temos um isomorfismo $\nu: \mathfrak{h} \longrightarrow \mathfrak{h}^{*}$ definido por

$$
\left\langle\nu(h), h_{1}\right\rangle=\left(h \mid h_{1}\right), h, h_{1} \in \mathfrak{h},
$$

e a forma bilinear $\left(. \mid\right.$.) induzida em $\mathfrak{h}^{*}$ (para definir a forma bilinear em $\mathfrak{h}^{*}$, podemos pensar em um caminho natural, que é $\left.(\alpha \mid \beta)=\left(\nu^{-1}(\alpha) \mid \nu^{-1}(\beta)\right), \forall \alpha, \beta \in \mathfrak{h}^{*}\right)$. 
Por (1.9), para todo $h \in \mathfrak{h}$

$$
\nu\left(\alpha_{i}^{V}\right)(h)=\left(\alpha_{i}^{V} \mid h\right)=\left\langle\alpha_{i}, h\right\rangle \epsilon_{i}=\epsilon_{i} \alpha_{i}(h), \text { o que implica } \nu\left(\alpha_{i}^{V}\right)=\epsilon_{i} \alpha_{i}, i=1, \ldots, n
$$

Por (1.11) deduzimos

$$
\left(\alpha_{i} \mid \alpha_{j}\right)=\left\langle\alpha_{i}, \alpha_{j}^{V}\right\rangle \epsilon_{j}^{-1}=a_{j i} \epsilon_{j}^{-1}=b_{i j}=a_{i j} \epsilon_{i}^{-1}=\left\langle\alpha_{j}, \alpha_{i}^{V}\right\rangle \epsilon_{i}^{-1}=\left(\alpha_{j} \mid \alpha_{i}\right) i, j=1, \ldots, n .
$$

Teorema 1.2.3. Seja $\mathfrak{g}(A)$ uma álgebra de Lie simetrizável. Fixemos uma decomposição (1.8) de A. Então, existe uma forma bilinear simétrica não degenerada a valores em $\mathbb{C}(. \mid$.) em $\mathfrak{g}(A)$ tal que:

(a) (.|.) é invariante, isto é, $([x, y] \mid z)=(x \mid[y, z])$, para todo $x, y, z \in \mathfrak{g}(A)$.

(b) (. . .) Ih é definida por (1.9) e (1.10) e é não degenerada.

(c) $\left(\mathfrak{g}_{\alpha} \mid \mathfrak{g}_{\beta}\right)=0$, se $\alpha+\beta \neq 0$.

(d) (. . . $\uparrow_{\mathfrak{g}_{\alpha}+\mathfrak{g}_{-\alpha}}$ é não degenerada para $\alpha \neq 0$, e consequentemente $\mathfrak{g}_{\alpha}$ e $\mathfrak{g}_{-\alpha}$ são pareados de forma não degenerada por (.|.).

(e) $[x, y]=(x \mid y) \nu^{-1}(\alpha)$, para $x \in \mathfrak{g}_{\alpha}, y \in \mathfrak{g}_{-\alpha}, \alpha \in \Delta$.

(vide [14, p.17-19])

Dada uma matriz $A$ simetrizável, a forma bilinear simétrica não degenerada invariante (.| .) em $\mathfrak{g}(A)$ fornecida pelo teorema anterior depende da decomposição fixada para $A$ e do subespaço de $\mathfrak{h}$ complementar a $\mathfrak{h}^{\prime}$. Contudo, uma vez fixados a decomposição e o subespaço, se definirmos uma forma (. . .) em h pelas relações (1.9),(1.10) e (1.11), podemos provar que existe uma única forma bilinear simétrica não degenerada invariante em $\mathfrak{g}(A)$ que estende a forma definida em $\mathfrak{h}$ por essas relações. De forma mais geral, temos o seguinte teorema:

Teorema 1.2.4. Seja A uma matriz simetrizável e $\mathfrak{g}(A)$ a álgebra de Lie associada a ela. Sejam $(. \mid .)_{1}$ e $(. \mid .)_{2}$ duas formas bilineares simétricas não degeneradas invariantes sobre $\mathfrak{g}(A)$. Se as restrições de $(. \mid .)_{1}$ e $(. \mid .)_{2}$ a $\mathfrak{h}$ coincidem, então $(. \mid .)_{1}=(. \mid .)_{2}$ em $\mathfrak{g}(A)$.

Demonstração. É suficiente provar que para todo $\alpha, \beta \in \Delta \cup\{0\}$, temos

$$
(x \mid y)_{1}=(x \mid y)_{2} \text {, para todo } x \in \mathfrak{g}_{\alpha} \text { e } y \in \mathfrak{g}_{\beta} .
$$

Se $\alpha+\beta \neq 0$, pelo item (c) do Teorema 1.2.3, $(x \mid y)_{1}=0=(x \mid y)_{2}$, para todo $x \in \mathfrak{g}_{\alpha}$ e $y \in \mathfrak{g}_{\beta}$.

Se $\alpha+\beta=0$, então $\beta=-\alpha$; se $\alpha=\beta=0$, pela hipótese do teorema, $(x \mid y)_{1}=(x \mid y)_{2}$, para $x, y \in \mathfrak{g}_{0}=\mathfrak{h}$.

Se $\alpha \neq 0$, pelo item (e) do Teorema 1.2.3, $[x, y]=(x \mid y)_{1} \nu^{-1}(\alpha)=(x \mid y)_{2} \nu^{-1}(\alpha)$, para $x \in \mathfrak{g}_{\alpha}$ e $y \in \mathfrak{g}_{-\alpha}$. 
Observação 1.2.5. Suponhamos agora que $A=\left(a_{i j}\right)$ seja uma matriz de Cartan generalizada simetrizável. Podemos fixar uma decomposição

$$
A=\operatorname{diag}\left(\epsilon_{1}, \ldots, \epsilon_{n}\right)\left(b_{i j}\right)_{i, j=1}^{n}
$$

em que $\epsilon_{i}$ são números racionais positivos, para todo $i=1, \ldots, n$, e $\left(b_{i j}\right)$ é uma matriz simétrica com entradas no conjunto dos números racionais.

Notemos que uma decomposição como em (1.14) sempre existe, pois é equivalente a um sistema de equações lineares homogêneas e desigualdades sobre $\mathbb{Q} \operatorname{com} \epsilon_{i}^{-1}$ e $b_{i j}$ não conhecidos:

$$
\epsilon_{i}^{-1} \neq 0 ; \operatorname{diag}\left(\varepsilon_{1}^{-1}, \ldots, \epsilon_{n}^{-1}\right) A=\left(b_{i j}\right) ; b_{i j}=b_{j i}
$$

Como $A$ é simetrizável, o sistema tem solução em $\mathbb{C}$; consequentemente, tem solução em $\mathbb{Q}$.

Podemos assumir que $A$ seja também indecomponível. Então, para todo $1<j \leq n$ existe uma sequência $1=i_{1}<i_{2}<\ldots<i_{k-1}<i_{k}=j$ tal que $a_{i_{s}, i_{s+1}}<0$. Temos:

$$
a_{i_{s}, i_{s+1}} \epsilon_{i_{s+1}}=a_{i_{s+1}, i_{s}} \epsilon_{i_{s}}(s=1, \ldots, k-1),
$$

implicando que $\epsilon_{j} \epsilon_{1}>0$, para todo $j=1, \ldots, n$, o que nos permite escolher todos os $\epsilon_{j}$ positivos, obtendo o resultado desejado.

Além disso, se $A$ é indecomponível, a matriz $\operatorname{diag}\left(\epsilon_{1}, \ldots, \epsilon_{n}\right)$ é unicamente determinada por (1.14), a menos de um fator constante.

Fixando uma forma simétrica bilinear não degenerada (. . .), associada à decomposição (1.14), por (1.13), temos:

$$
\begin{aligned}
\left(\alpha_{i} \mid \alpha_{i}\right) & =a_{i i} \epsilon_{i}^{-1}=2 \epsilon_{i}^{-1}>0, i=1, \ldots, n \\
\left(\alpha_{i} \mid \alpha_{j}\right) & =a_{i j} \epsilon_{i}^{-1} \leq 0 \\
\alpha_{i}^{V} & =\nu^{-1}\left(\alpha_{i} \epsilon_{i}\right)=\epsilon_{i} \nu^{-1}\left(\alpha_{i}\right)=\frac{2}{\left(\alpha_{i} \mid \alpha_{i}\right)} \nu^{-1}\left(\alpha_{i}\right) .
\end{aligned}
$$

Com as relações acima, obtemos a expressão usual para a matriz de Cartan generalizada:

$$
A=\left(\frac{2\left(\alpha_{i} \mid \alpha_{j}\right)}{\left(\alpha_{i} \mid \alpha_{i}\right)}\right)_{i, j=1}^{n}
$$

A forma bilinear $(. \mid$.) em $\mathfrak{g}(A)$ fornecida pelo Teorema 1.2 .3 e satisfazendo (1.15) é denominada forma invariante padrão.

\subsection{Representações Integráveis e o Grupo de Weyl}

A partir da Definição 1.1.1, é imediato que a transposta de uma matriz de Cartan generalizada é ainda uma matriz do mesmo tipo; e como já vimos anteriormente, se $\left(\mathfrak{h}, \Pi, \Pi^{V}\right)$ é uma realização de uma matriz $A$, então $\left(\mathfrak{h}^{*}, \Pi^{V}, \Pi\right)$ é uma realização de $A^{t}$. Assim, do mesmo modo que construímos a álgebra de Kac-Moody $\mathfrak{g}(A)$ associada a $A$, podemos construir uma álgebra de Kac-Moody $\mathfrak{g}\left(A^{t}\right)$ associada a $A^{t}$, e elas serão denominadas dual uma da outra. 
O reticulado de raízes dual de $\mathfrak{g}(A)$

$$
Q^{V}:=\sum_{i=1}^{n} \mathbb{Z} \alpha_{i}^{V}
$$

é exatamente o reticulado de raízes de $\mathfrak{g}\left(A^{t}\right)$.

O sistema de raízes de $\mathfrak{g}\left(A^{t}\right)$, denotado por $\Delta^{V} \subset Q^{V}$, é o sistema de raízes dual.

Seja $\mathfrak{g}(A)$ uma álgebra de Kac-Moody, e sejam $e_{i}, f_{i}(i=1, \ldots, n)$ seus geradores de Chevalley. Seja $\mathfrak{g}_{(i)}=\mathbb{C} e_{i}+\mathbb{C} \alpha_{i}^{V}+\mathbb{C} f_{i}$; então, $\mathfrak{g}_{(i)}$ é isomorfo a $\mathfrak{s l}(2, \mathbb{C})$, com base padrão $\left\{e_{i}, \alpha_{i}^{V}, f_{i}\right\}$, e o isomorfismo é dado por

$$
f_{i} \longmapsto\left(\begin{array}{cc}
0 & 0 \\
1 & 0
\end{array}\right), \alpha_{i}^{V} \longmapsto\left(\begin{array}{cc}
1 & 0 \\
0 & -1
\end{array}\right) \text { e } e_{i} \longmapsto\left(\begin{array}{cc}
0 & 1 \\
0 & 0
\end{array}\right) .
$$

Algumas vezes, torna-se bastante útil olhar para essa cópia isomórfica de $\mathfrak{s l}(2, \mathbb{C})$ que existe em $\mathfrak{g}(A)$, pois podemos aplicar resultados que são válidos em $\mathfrak{s l}(2, \mathbb{C})$; um exemplo disso é a demonstração das bem conhecidas relações entre os geradores de Chevalley de $\mathfrak{g}(A)$ :

$$
\left(a d e_{i}\right)^{1-a_{i j}} e_{j}=0 ;\left(a d f_{i}\right)^{1-a_{i j}} f_{j}=0, \text { se } i \neq j
$$

Para efetuar a demonstração, utilizaremos o seguinte resultado:

Lema 1.3.1. Seja $V$ um $\mathfrak{s l}(2, \mathbb{C})$-módulo e seja $v \in V$ tal que

$$
h(v)=\lambda v, \text { para algum } \lambda \in \mathbb{C}
$$

Seja $v_{j}=(j !)^{-1} f^{j}(v)$. Então,

$$
h\left(v_{j}\right)=(\lambda-2 j) v_{j}
$$

Se, além disso, $e(v)=0$, então

$$
e\left(v_{j}\right)=(\lambda-j+1) v_{j-1}
$$

(vide [14, p.31]).

Demonstração. (Relações) Faremos aqui a demonstração da segunda igualdade; a primeira pode ser obtida fazendo uso da involução de Chevalley.

Para aplicar o lema, iremos considerar $\mathfrak{g}(A)$ como um $\mathfrak{g}_{(i)}$-módulo pela restrição da representação adjunta; fazendo a identificação $e_{i}=e, f_{i}=f, \alpha_{i}^{V}=h$, tomando $v=f_{j}, \theta_{i j}=\left(a d f_{i}\right)^{1-a_{i j}} f_{j}$, e utilizando as relações (1.1), temos:

$$
\alpha_{i}^{V}(v)=-a_{i j} v ; e_{i}(v)=0, \text { se } i \neq j
$$

fazendo agora $\lambda=-a_{i j}$ e $j=1-a_{i j}$, podemos aplicar o Lema, e junto com as propriedades (C1) 
e (C2) da matriz $A$ :

$$
\left[e_{i}, \theta_{i j}\right]=\left(1-a_{i j}\right) !\left[e_{i}, v_{1-a_{i j}}\right]=\left(1-a_{i j}\right)\left(-a_{i j}-\left(1-a_{i j}\right)+1\right)\left(a d f_{i}\right)^{-a_{i j}} f_{j}=0, \text { se } i \neq j
$$

Temos também que se $k \neq i, k \neq j$, fazendo uso da identidade de Jacobi e pelas relações (1.1), $e_{k}$ comuta com $\theta_{i j}$; o mesmo ocorre se $k=j$ e $a_{i j} \neq 0$. Vamos agora analisar o caso $k=j$ e $a_{i j}=0$ :

$$
\left[e_{j}, \theta_{i j}\right]=\left[e_{j},\left[f_{i}, f_{j}\right]\right]=\left[\left[f_{j}, e_{j}\right], f_{i}\right]+\left[\left[e_{j}, f_{i}\right], f_{j}\right]=-\left[\alpha_{j}^{V}, f_{i}\right]=\left\langle\alpha_{i}, \alpha_{j}^{V}\right\rangle f_{i}=a_{j i} f_{i}=0,
$$

pela propriedade (C3) da matriz $A$ e utilizando a identidade de Jacobi.

Pelo Lema (1.5) em ([14, p.10]), se $a \in \mathfrak{n}_{-}$é tal que $\left[a, e_{i}\right]=0$, para todo $i=1, \ldots, n$, então $a=0$. Assim, $\theta_{i j}=0$.

Definição 1.3.2. Sejam $\mathfrak{g}$ uma álgebra de Lie e $V$ um módulo sobre $\mathfrak{g}$. Diz-se que um elemento $x \in \mathfrak{g}$ é localmente nilpotente em $V$ se, para cada $v \in V$, existe um inteiro positivo $N$ tal que $x^{N}(v)=0$.

Lema 1.3.3. Seja $\mathfrak{g}(A)$ uma álgebra de Kac-Moody. Então, $\left(a d e_{i}\right)$ e $\left(a d f_{i}\right)$ são localmente nilpotentes em $\mathfrak{g}(A)$, para todo $i \in\{1, \ldots, n\}$.

Demonstração. Para mostrar o que desejamos, basta verificar que $\left(a d e_{i}\right)$ e $\left(a d f_{i}\right)$ são localmente nilpotentes no sistema de geradores $\left\{\mathfrak{h}, e_{i}, f_{i}, i=1, \ldots, n\right\}$, pois pelo Lema 3.4(a) em ([14, p.32-33]), para qualquer elemento $x \in \mathfrak{g}$, com $\mathfrak{g}$ uma álgebra de Lie qualquer, (ad $x$ ) é localmente nilpotente em $\mathfrak{g}$ se o for em algum sistema de geradores. Assim, utilizando as relações (1.1),temos:

$$
\begin{aligned}
\left(a d e_{i}\right)^{2} h & =\left[e_{i},\left[e_{i}, h\right]\right]=\left[e_{i},-\left\langle\alpha_{i}, h\right\rangle e_{i}\right]=-\left\langle\alpha_{i}, h\right\rangle\left[e_{i}, e_{i}\right]=0, \\
\left(a d f_{i}\right)^{2} h & =\left[f_{i},\left[f_{i}, h\right]\right]=\left[f_{i},\left\langle\alpha_{i}, h\right\rangle f_{i}\right]=\left\langle\alpha_{i}, h\right\rangle\left[f_{i}, f_{i}\right]=0 \forall h \in \mathfrak{h}, \\
\left(a d e_{i}\right)^{3} f_{i} & =\left[e_{i},\left[e_{i},\left[e_{i}, f_{i}\right]\right]\right]=\left[e_{i},\left[e_{i}, \alpha_{i}^{V}\right]\right]=\left[e_{i},-\left\langle\alpha_{i}, \alpha_{i}^{V}\right\rangle e_{i}\right]=0=\left(a d e_{i}\right)^{1+2} f_{i}=\left(\text { ad } e_{i}\right)^{1+\left|a_{i i}\right|} f_{i}, \\
\left(\text { ad } e_{i}\right) f_{j} & =0, \text { se } i \neq j, \text { e }\left(a d e_{i}\right) e_{i}=0
\end{aligned}
$$

e pelas relações $(1.18),\left(a d e_{i}\right)$ é localmente nilpotente em $e_{j}$ e $\left(a d f_{i}\right)$ é localmente nilpotente em $f_{j}$, para $i \neq j,(i=1, \ldots, n)$. Podemos então concluir que

$$
\left(a d e_{i}\right)^{\left|a_{i j}\right|+1} x=0=\left(a d f_{i}\right)^{\left|a_{i j}\right|+1} x
$$

se $x=e_{j}$ ou $x=f_{j}$ e $\left(a d e_{i}\right)^{2} h=0=\left(a d f_{i}\right)^{2} h$, se $h \in \mathfrak{h}$, obtendo o resultado desejado.

Definição 1.3.4. Sejam $\mathfrak{g}(A)$ uma álgebra de Kac-Moody e $\mathfrak{h}$ sua subálgebra de Cartan. Diremos que um $\mathfrak{g}(A)$-módulo $V$ é $\mathfrak{h}$-diagonalizável se

$$
V=\bigoplus_{\lambda \in \mathfrak{h}^{*}} V_{\lambda}
$$

em que $V_{\lambda}=\{v \in V \mid h(v)=\langle\lambda, h\rangle v$, para todo $h \in \mathfrak{h}\} . V_{\lambda}$ é denominado epaço de peso, $\lambda \in \mathfrak{h}^{*}$ é denominado peso se $V_{\lambda} \neq\{0\}$, a dimensão de $V_{\lambda}$ é denominada multiplicidade de $\lambda$ e é 
denotada por mult ${ }_{V} \lambda$.

Definição 1.3.5. Um módulo $\mathfrak{h}$-diagonalizável sobre uma álgebra de Kac-Moody $\mathfrak{g}(A)$ é denominado integrável se todos os $e_{i}, f_{i}(i=1, \ldots, n)$ são localmente nilpotentes em $V$.

Observação 1.3.6. A partir do Lema 1.3.3, podemos concluir que o módulo subjacente da representação adjunta de uma álgebra de Kac-Moody é um módulo integrável.

Proposição 1.3.7. Seja $V$ um $\mathfrak{g}(A)$-módulo integrável.

(a) Como um $\mathfrak{g}_{(i)}$-módulo, $V$ se decompõe em uma soma direta de módulos $\mathfrak{h}$-invariantes irredutíveis de dimensão finita.

(b) Seja $\lambda \in \mathfrak{h}^{*}$ um peso de $V$ e seja $\alpha_{i}$ uma raiz simples de $\mathfrak{g}(A)$. Denote por $M$ o conjunto de todos os $t \in \mathbb{Z}$ tais que $\lambda+t \alpha_{i}$ é um peso de $V$, e seja $m_{t}=$ mult $_{V}\left(\lambda+t \alpha_{i}\right)$. Então,

(i) $M$ é o intervalo fechado de inteiros $[-p, q]$, em que $p$ e q são ambos ou inteiros não negativos ou $\infty$ e $p-q=\left\langle\lambda, \alpha_{i}^{V}\right\rangle$, quando $p$ e q são finitos; se mult $_{V} \lambda<\infty$, então $p$ e q são finitos;

(ii) $e_{i}: V_{\lambda+t \alpha_{i}} \longrightarrow V_{\lambda+(t+1) \alpha_{i}}$ é injetiva para $t \in\left[-p,-\frac{1}{2}\left\langle\lambda, \alpha_{i}^{V}\right\rangle\right)$; em particular, a função $t \longmapsto m_{t}$ é crescente nesse intervalo;

(iii) a função $t \longmapsto m_{t}$ é simétrica com respeito a $t=-\frac{1}{2}\left\langle\lambda, \alpha_{i}^{V}\right\rangle$;

(iv) se tanto $\lambda$ como $\lambda+\alpha_{i}$ são pesos, então $e_{i}\left(V_{\lambda}\right) \neq 0$.

(vide [14, p.34]).

O Corolário abaixo tem importantes aplicações práticas.

Corolário 1.3.8. (a) $S e \lambda$ é um peso de um $\mathfrak{g}(A)$-módulo integrável $V$ e $\lambda+\alpha_{i}$ (respectivamente $\left.\lambda-\alpha_{i}\right)$ não é um peso, então $\left\langle\lambda, \alpha_{i}^{V}\right\rangle \geq 0$ (respectivamente $\left\langle\lambda, \alpha_{i}^{V}\right\rangle \leq 0$ ).

(b) Se $\lambda$ é um peso de $V$, então $\lambda-\left\langle\lambda, \alpha_{i}^{V}\right\rangle \alpha_{i}$ é também um peso de mesma multiplicidade.

Definição 1.3.9. Seja $\mathfrak{g}(A)$ uma álgebra de Kac-Moody. Para cada $i=1, \ldots, n$, definimos a reflexãa fundamental $r_{i}$ do espaço $\mathfrak{h}^{*}$ por

$$
r_{i}(\lambda)=\lambda-\left\langle\lambda, \alpha_{i}^{V}\right\rangle \alpha_{i}, \lambda \in \mathfrak{h}^{*}
$$

Em particular, $r_{i}\left(\alpha_{j}\right)=\alpha_{j}-\left\langle\alpha_{j}, \alpha_{i}^{V}\right\rangle \alpha_{i}=\alpha_{j}-a_{i j} \alpha_{i} \in Q\left(r_{i}\left(\alpha_{i}\right)=-\alpha_{i}\right)$, o que nos dá $r_{i} Q \subset Q$. Como o conjunto $\left\{\alpha_{1}, \ldots, \alpha_{n}\right\}$ é um subconjunto linearmente independente de $\mathfrak{h}^{*}$, podemos completálo para obter uma base. Para cada i fixado, escrevendo a matriz de $r_{i}$ nessa base, teremos que detr $_{i}=-1$, e ela é sua própria inversa. Assim, $r_{i}^{2}=I d$.

$O$ conjunto dos pontos fixos de $r_{i}$ é $T_{i}=\left\{\lambda \in \mathfrak{h}^{*} \mid\left\langle\lambda, \alpha_{i}^{V}\right\rangle=0\right\}$, que é um hiperplano de $\mathfrak{h}^{*}$. Assim, $r_{i}$ é de fato uma reflexão, para todo $i=1, \ldots, n$.

O subgrupo $W$ dos automorfismos de $\mathfrak{h}^{*}$ gerado por todas as reflexões fundamentais é denominado grupo de Weyl de $\mathfrak{g}(A)$. 
A reflexão fundamental $r_{i}$ induz uma reflexão fundamental dual $r_{i}^{*}$ em $\mathfrak{h}$ tal que

$$
\left\langle r_{i}^{*}(h), \lambda\right\rangle=\left\langle h, r_{i}(\lambda)\right\rangle \forall h \in \mathfrak{h}, \lambda \in \mathfrak{h}^{*}
$$

substituindo (1.19) na equação acima, temos:

$$
\begin{aligned}
\left\langle r_{i}^{*}(h), \lambda\right\rangle & =\left\langle h, \lambda-\left\langle\lambda, \alpha_{i}^{V}\right\rangle \alpha_{i}\right\rangle \\
& =\langle h, \lambda\rangle-\left\langle h,\left\langle\lambda, \alpha_{i}^{V}\right\rangle \alpha_{i}\right\rangle \\
& =\langle h, \lambda\rangle-\left\langle\lambda, \alpha_{i}^{V}\right\rangle\left\langle h, \alpha_{i}\right\rangle \\
& =\left\langle\lambda, h-\left\langle h, \alpha_{i}\right\rangle \alpha_{i}^{V}\right\rangle
\end{aligned}
$$

Consequentemente, $r_{i}^{*}(h)=h-\left\langle h, \alpha_{i}\right\rangle \alpha_{i}^{V}$, pois o pareamento $\langle$,$\rangle é uma forma bilinear não degene-$ rada.

Proposição 1.3.10. (a) Seja $V$ um módulo integrável sobre uma álgebra de Kac-Moody $\mathfrak{g}(A)$.

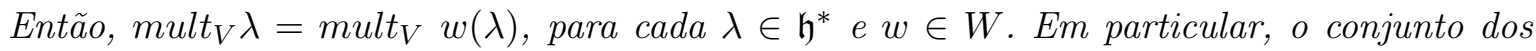
pesos de $V$ é $W$-invariante.

(b) O sistema de raízes $\Delta$ de $\mathfrak{g}(A)$ é $W$-invariante, e mult $\alpha=$ mult $w(\alpha)$ para cada $\alpha \in \Delta e$ $w \in W$.

Demonstração. (a) Seja $\lambda \in \mathfrak{h}^{*}$. Se $\lambda$ for um peso de $V$, então $r_{i}(\lambda)$ também o será, e ambos terão a mesma multiplicidade, pelo item (b) do Corolário 1.3.8, para todo $i=1, \ldots, n$, e teremos que o resultado é válido para todo $w \in W$. Por outro lado, se $\lambda$ não for um peso de $V$, então $r_{i}(\lambda)$ também não será, para nenhum $i=1, \ldots, n$, ou teríamos uma contradição, já que $r_{i}^{2}=1$. Portanto, mult $_{V} \lambda=0=$ mult $_{V}\left(\lambda-\left\langle\lambda, \alpha_{i}^{V}\right\rangle \alpha_{i}\right)$, e temos o resultado desejado para todo $\lambda \in \mathfrak{h}^{*}$.

(b) Para mostrar que $\Delta$ é $W$-invariante, e mult $\alpha=$ mult $w(\alpha)$, para todo $\alpha \in \Delta$ e todo $w \in W$, basta mostrar que isso ocorre com relação a $r_{i}$ para todo $i=1, \ldots n$. Pela Observação 1.3.6, podemos aplicar o Corolário 1.3.8(b) para $\mathfrak{g}(A)$. O sistema de raízes $\Delta$ é, em particular um conjunto de pesos não nulos de $\mathfrak{g}(A)$. Então, pelo Corolário 1.3.8(b), temos que para todo $\alpha \in \Delta, r_{i}(\alpha)$ é ainda um peso de $\mathfrak{g}(A)$, com a mesma multiplicidade de $\alpha$. Como $\alpha \neq 0$, $r_{i}(\alpha) \neq 0$, já que para cada $i=1, \ldots, n, r_{i}$ é uma bijeção, o que nos fornece o resultado desejado.

Observação 1.3.11. Consideremos $\alpha \in \Delta_{+} \backslash\left\{\alpha_{i}\right\}, i=1, \ldots, n$. Como cada raiz é positiva ou negativa, então (1.5) implica que se $\alpha+t \alpha_{i}$ for uma raiz, com $t \in \mathbb{Z}$, será necessariamente uma raiz positiva, isto é, $\left(\alpha+\mathbb{Z} \alpha_{i}\right) \cap \Delta \subset \Delta_{+}$.

Portanto, se $\alpha \in \Delta_{+}$e $r_{i}(\alpha)<0$, então $\alpha=\alpha_{i}$. Logo, $\Delta_{+} \backslash\left\{\alpha_{i}\right\}$ é $r_{i}$-invariante.

Proposição 1.3.12. Seja A uma matriz de Cartan generalizada simetrizável e seja (.| .) a forma bilinear invariante padrão em $\mathfrak{g}(A)$. A restrição de (.| .) a h⿱㇒ $\mathfrak{h}^{*}$ é $W$-invariante. 
Demonstração. Sejam $\lambda, \rho \in \mathfrak{h}^{*}$. Então, para todo $i=1, \ldots, n$, utilizando a equação (1.17) e a definição de $\nu$, temos:

$$
\begin{aligned}
\left(r_{i}(\lambda) \mid r_{i}(\rho)\right) & =\left(\lambda-\left\langle\lambda, \alpha_{i}^{V}\right\rangle \alpha_{i} \mid \rho-\left\langle\rho, \alpha_{i}^{V}\right\rangle \alpha_{i}\right) \\
& =(\lambda \mid \rho)-\left\langle\rho, \alpha_{i}^{V}\right\rangle\left(\lambda \mid \alpha_{i}\right)-\left\langle\lambda, \alpha_{i}^{V}\right\rangle\left(\rho \mid \alpha_{i}\right)+\left\langle\lambda, \alpha_{i}^{V}\right\rangle\left\langle\rho, \alpha_{i}^{V}\right\rangle\left(\alpha_{i} \mid \alpha_{i}\right) \\
& =(\lambda \mid \rho)-\left\langle\rho, \alpha_{i}^{V}\right\rangle\left(\nu^{-1}(\lambda) \mid \nu^{-1}\left(\alpha_{i}\right)\right)-\left\langle\lambda, \alpha_{i}^{V}\right\rangle\left(\nu^{-1}(\rho) \mid \nu^{-1}\left(\alpha_{i}\right)\right)+\left\langle\lambda, \alpha_{i}^{V}\right\rangle\left\langle\rho, \alpha_{i}^{V}\right\rangle\left(\alpha_{i} \mid \alpha_{i}\right) \\
& =(\lambda \mid \rho)-\epsilon_{i}^{-1}\left\langle\rho, \alpha_{i}^{V}\right\rangle\left\langle\nu \nu^{-1}(\lambda), \alpha_{i}^{V}\right\rangle-\epsilon_{i}^{-1}\left\langle\lambda, \alpha_{i}^{V}\right\rangle\left\langle\nu \nu^{-1}(\rho), \alpha_{i}^{V}\right\rangle+\left\langle\lambda, \alpha_{i}^{V}\right\rangle\left\langle\rho, \alpha_{i}^{V}\right\rangle\left(\alpha_{i} \mid \alpha_{i}\right) \\
& =(\lambda \mid \rho)-2 \epsilon_{i}^{-1}\left\langle\rho, \alpha_{i}^{V}\right\rangle\left\langle\lambda, \alpha_{i}^{V}\right\rangle+\left\langle\lambda, \frac{2}{\left(\alpha_{i} \mid \alpha_{i}\right)} \nu^{-1}\left(\alpha_{i}\right)\right\rangle\left\langle\rho, \alpha_{i}^{V}\right\rangle\left(\alpha_{i} \mid \alpha_{i}\right) \\
& =(\lambda \mid \rho)-2 \epsilon_{i}^{-1}\left\langle\rho, \alpha_{i}^{V}\right\rangle\left\langle\lambda, \alpha_{i}^{V}\right\rangle+2 \epsilon_{i}^{-1}\left\langle\lambda, \alpha_{i}^{V}\right\rangle\left\langle\rho, \alpha_{i}^{V}\right\rangle \\
& =(\lambda \mid \rho) .
\end{aligned}
$$

\subsection{Uma Classificação das Matrizes de Cartan Generalizadas}

Nesta seção, nosso objetivo é enunciar os principais resultados que nos levarão à classificação das matrizes de Cartan generalizadas dos tipos finito e afim. Essa classificação será efetuada utilizando-se o diagrama de Dynkin.

Trabalharemos com matrizes $A=\left(a_{i j}\right)$ reais $n \times n$, que satisfaçam as seguintes propriedades:

(m1) A é indecomponível;

$(\mathrm{m} 2) a_{i j} \leq 0$, para $i \neq j$

(m3) $a_{i j}=0$ implica $a_{j i}=0$.

Podemos assumir, sem perda de generalidade, que uma matriz de Cartan generalizada satisfaça (m1).

O próximo teorema é o resultado mais importante desta seção.

Teorema 1.4.1. Seja A uma matriz real $n \times n$ satisfazendo $(m 1),(m 2)$ e $(m 3)$. Então, uma e apenas uma das três seguintes possibilidades vale para ambas $A$ e $A^{t}$ :

(Fin) det $A \neq 0$; existe $u>0$ tal que $A u>0 ; A v \geq 0$ implica $v>0$ ou $v=0$;

(Aff) $n$-posto $A=1$; existe $u>0$ tal que $A u=0 ; A v \geq 0$ implica $A v=0$;

(Ind) existe $u>0$ tal que $A u<0 ; A v \geq 0, v \geq 0$ implica $v=0$,

em que u e $v$ são vetores coluna com entradas em $\mathbb{R}$.

(vide [14, p.48-49])

Diremos que $A$ é do tipo finito, afim ou indefinido, conforme satisfaça as condições (Fin), (Aff) ou (Ind), respectivamente.

Ao associarmos a cada uma dessas matrizes o correspondente diagrama de Dynkin, o mesmo acompanhará essa classificação.

De acordo com a Proposição 4.7 (vide [14, p.51]), temos o seguinte resumo dos resultados para uma matriz de Cartan generalizada indecomponível $A$ : 
(a) $A$ é do tipo finito se e somente se todos os seus menores principais são positivos.

(b) $A$ é do tipo afim se e somente se todos os seus menores principais próprios são positivos e $\operatorname{det} A=0$.

(c) $A$ é do tipo afim se e somente se existe $\delta>0$ tal que $A \delta=0$; tal $\delta$ é único a menos de multiplicação por uma constante.

Os diagramas de Dynkin, que estudaremos a seguir, permitem-nos listar todas as matrizes de Cartan generalizadas dos tipos finito e afim.

Teorema 1.4.2. (a) Os diagramas de Dynkin de todas as matrizes de Cartan generalizadas do tipo finito estão listados na tabela Fin.

(b) Os diagramas de Dynkin de todas as matrizes de Cartan generalizadas do tipo afim estão listados nas tabelas Aff 1, Aff 2 e Aff 3 (todos eles possuem l +1 vértices).

(c) A numeração nos diagramas das tabelas do item (b) são as coordenadas do único vetor $\delta=\left(a_{o}, a_{1}, \ldots, a_{l}\right)^{t}$ tal que $A \delta=0$ e os $a_{i}$ são inteiros positivos relativamente primos, com $i=0, \ldots, l$.

(vide [14, p. 52-53])

Observação 1.4.3. A enumeração dos vértices nos diagramas de Dynkin é feita da seguinte forma:

(1) Na tabela Fin, os vértices dos diagramas estão enumerados pelos símbolos $\alpha_{1}, \ldots, \alpha_{l}$;

(2) Cada diagrama $X_{l}^{(1)}$ da tabela Aff 1 é obtido do diagrama $X_{l}$ pela adição de um vértice, enumerado por $\alpha_{0}$, mantendo o restante da enumeração já existente nos vértices originais.

(3) Os números entre parênteses na tabela Fin são $\operatorname{det} A$.

(4) As etiquetas numéricas nas tabelas Aff são os coeficientes de uma dependência linear entre as colunas de A.

Exemplo 1.4.4. Consideremos uma matriz $A$ do tipo $F_{4}$ da tabela (Fin). Temos então que

$$
A=\left(\begin{array}{cccc}
2 & -1 & 0 & 0 \\
-1 & 2 & -1 & 0 \\
0 & -2 & 2 & -1 \\
0 & 0 & -1 & 2
\end{array}\right)
$$

Assim, a matriz $B$ do tipo $F_{4}^{(1)}$ da tabela (Aff1), obtida através dela é da forma

$$
B=\left(\begin{array}{ccccc}
2 & -1 & 0 & 0 & 0 \\
-1 & 2 & -1 & 0 & 0 \\
0 & -1 & 2 & -1 & 0 \\
0 & 0 & -2 & 2 & -1 \\
0 & 0 & 0 & -1 & 2
\end{array}\right)
$$


Se denotarmos as colunas de $B$ por $c 0, c 1, \ldots, c 4$, notemos que $c 0+2 c 1+3 c 2+4 c 3+2 c 4=0$, mostrando que as etiquetas numéricas são realmente coeficientes de uma dependência linear entre as colunas de $B$. Notemos também que o posto de $A$ é 4 , sendo seu determinante diferente de zero; já o posto de $B$ é 4 e seu determinante é zero.

Observação 1.4.5. As álgebras de Kac-Moody $\mathfrak{g}(A)$ associadas às matrizes de Cartan generalizadas indecomponíveis do tipo finito são álgebras de Lie simples de dimensão finita, conforme pode ser verificado em [14] . 


\begin{tabular}{|c|c|c|}
\hline$A_{l}$ & $\stackrel{\circ-}{\alpha_{1}}{ }_{\alpha_{2}}^{\circ-}$ & $\cdots-\underset{\alpha_{l-1}}{\circ} \alpha_{l}$ \\
\hline$B_{l}$ & $\stackrel{\circ}{\alpha_{1}}-\stackrel{\circ}{\alpha_{2}}$ & $\cdots-\underset{\alpha_{l-1}}{\circ} \stackrel{\circ}{\alpha_{l}}$ \\
\hline$C_{l}$ & $\stackrel{\circ-}{\alpha_{1}}{ }_{\alpha_{2}}^{\circ-}$ & $\begin{array}{c}\cdots-\underset{\alpha_{l-1}}{\circ} \alpha_{l} \\
\circ \alpha_{l}\end{array}$ \\
\hline$D_{l}$ & $\stackrel{\circ}{\circ}-\stackrel{\circ}{\alpha_{1}} \alpha_{2}$ & $\begin{array}{l}\cdots-\alpha_{l-2} \alpha_{l-1} \\
\circ \alpha_{6}\end{array}$ \\
\hline$E_{6}$ & $\stackrel{\circ-}{\circ} \alpha_{1}-$ & $\begin{array}{l}\stackrel{\circ}{\alpha_{3}} \alpha_{4}-\stackrel{\circ}{\circ} \alpha_{5} \\
\circ \alpha_{7}\end{array}$ \\
\hline$E_{7}$ & $\stackrel{\circ}{\alpha_{1}}-\underset{\alpha_{2}}{\circ}-$ & 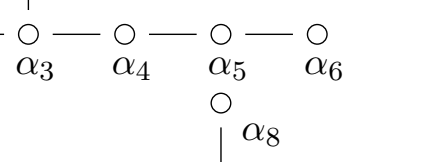 \\
\hline$E_{8}$ & $\stackrel{\circ-}{\alpha_{1}}{ }_{\alpha_{2}}^{\circ-}$ & $\stackrel{\circ}{\circ}-\underset{\alpha_{3}}{\circ}-\stackrel{\circ}{\circ}-\underset{\alpha_{5}}{\circ}-\underset{\alpha_{6}}{\circ}-\stackrel{\circ}{\circ}$ \\
\hline$F_{4}$ & $\stackrel{\circ}{\alpha_{1}}-\stackrel{\circ}{\alpha_{2}} \Rightarrow$ & $\stackrel{\circ}{\circ}-\stackrel{\circ}{\alpha_{3}}{ }_{\alpha_{4}}$ \\
\hline$G_{2}$ & $\stackrel{\circ}{\circ} \underset{\alpha_{1}}{\Rightarrow} \stackrel{\circ}{\alpha_{2}}$ & \\
\hline
\end{tabular}

Tabela 1.1: Fin 


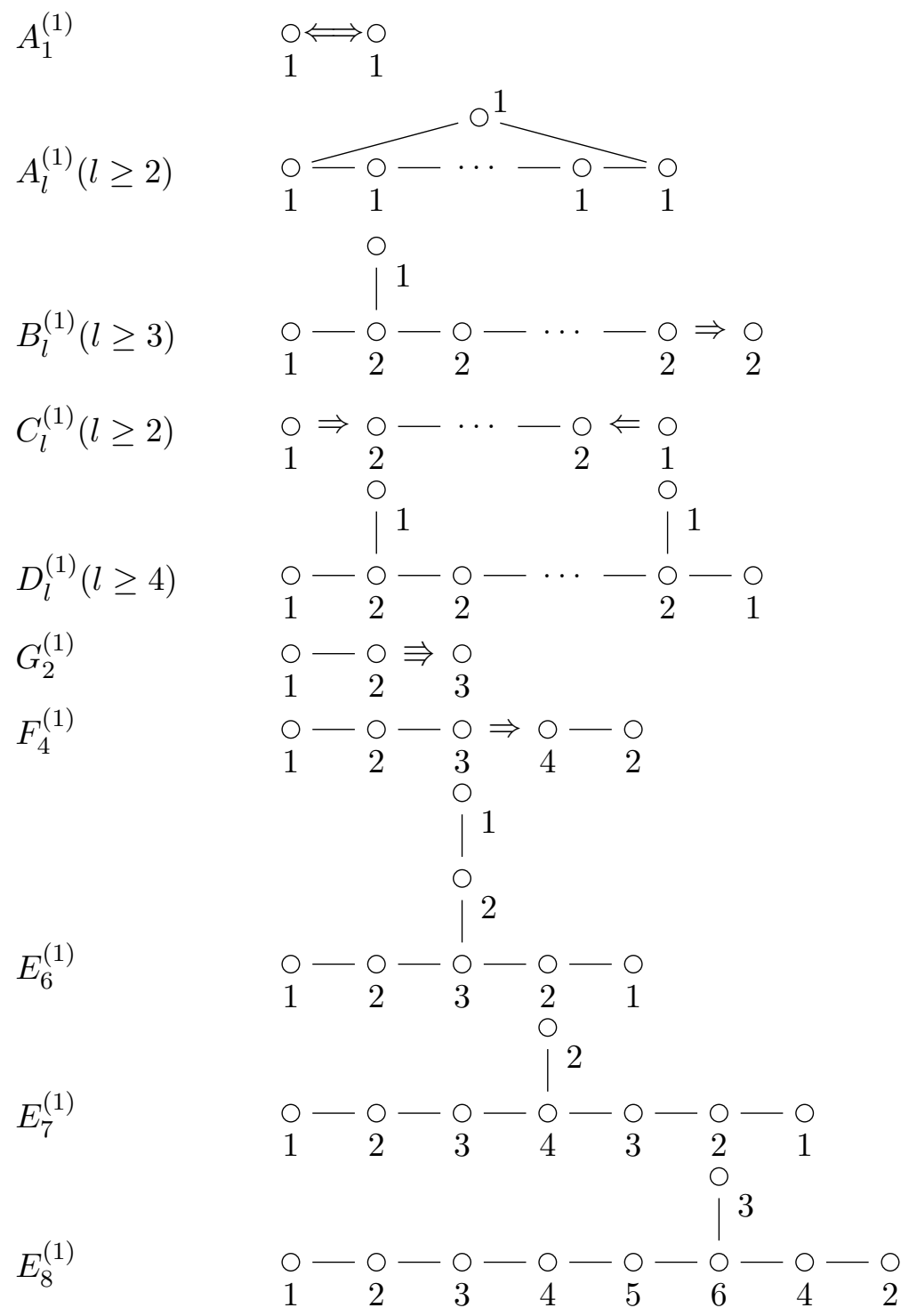

Tabela 1.2: Aff1 


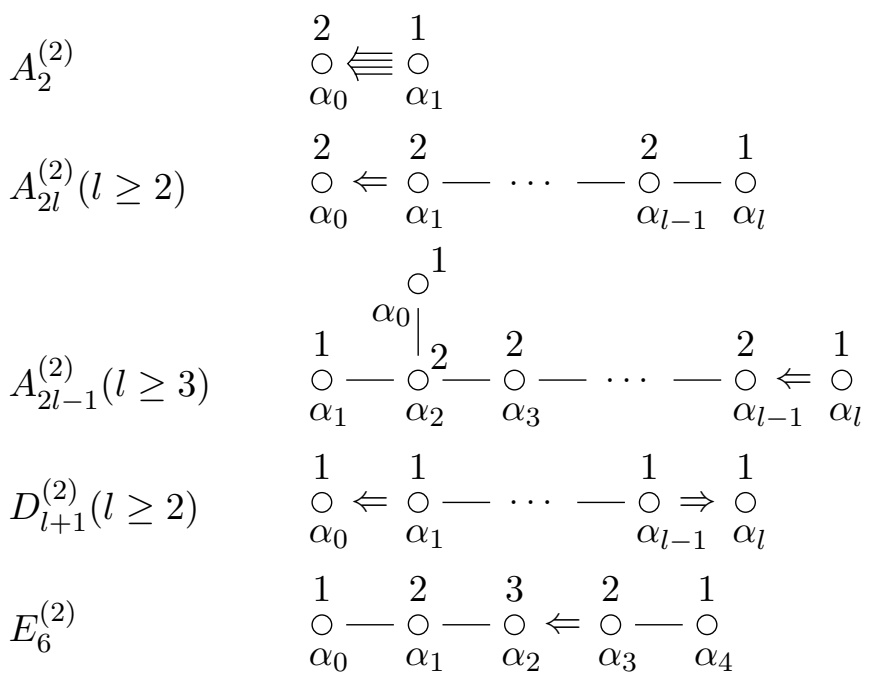

Tabela 1.3: Aff 2

$D_{4}^{(3)} \quad \stackrel{1}{\circ}-\stackrel{2}{\circ} \underset{\alpha_{0}}{\circ} \Leftarrow \stackrel{1}{\circ} \underset{\alpha_{2}}{\circ}$

Tabela 1.4: Aff 3 


\subsection{Raízes Reais e Raízes Imaginárias}

Nesta seção estudaremos o sistema de raízes $\Delta$ de uma álgebra de Kac-Moody $\mathfrak{g}(A)$, exibindo todas as raízes no caso em que essa álgebra for do tipo afim.

Definição 1.5.1. Uma raiz $\alpha \in \Delta$ é denominada real se existe $w \in W$ tal que $w(\alpha)$ é uma raiz simples. Os conjuntos de todas as raizes reais e das raízes reais positivas serão denotados respectivamente por $\Delta^{r e}$ e $\Delta_{+}^{r e}$.

Seja agora $\alpha \in \Delta^{r e}$. Então, $\alpha=w\left(\alpha_{i}\right)$ para algum $\alpha_{i} \in \Pi(i=1, \ldots, n)$ e $w \in W$. Podemos definir a raiz (real) dual $\alpha^{V}$ por $\alpha^{V}=w\left(\alpha_{i}^{V}\right)$.

Definimos também uma reflexão $r_{\alpha}$ com respeito a $\alpha \in \Delta^{r e}$ por

$$
r_{\alpha}(\lambda)=\lambda-\left\langle\lambda, \alpha^{V}\right\rangle \alpha, \lambda \in \mathfrak{h}^{*}
$$

Proposição 1.5.2. Seja $\alpha$ uma raiz real de uma ágebra de Kac-Moody $\mathfrak{g}(A)$. Então,

(a) mult $\alpha=1$;

(b) $k \alpha$ é uma raiz se e somente se $k= \pm 1$;

(c) Se $\beta \in \Delta$, então existem inteiros não negativos $p$ e q relacionados pela equação

$$
p-q=\left\langle\beta, \alpha^{V}\right\rangle
$$

tais que $\beta+k \alpha \in \Delta \cup\{0\}$ se e somente se $-p \leq k \leq q, k \in \mathbb{Z}$;

(d) Suponha que A é simetrizável e seja (. | .) a forma bilinear invariante padrão em $\mathfrak{g}(A)$. Então,

(i) $(\alpha \mid \alpha)>0$;

(ii) $\alpha^{V}=2 \nu^{-1}(\alpha) /(\alpha \mid \alpha)$;

(iii) se $\alpha=\sum_{i} k_{i} \alpha_{i}$, então $k_{i}\left(\alpha_{i} \mid \alpha_{i}\right) \in(\alpha \mid \alpha) \mathbb{Z}$.

(e) Devido a $\pm \alpha \notin \Pi$, existe $i$ tal que

$$
\left|h t r_{i}(\alpha)\right|<|h t \alpha|
$$

(vide [14, p.60]).

Seja $A$ uma matriz de Cartan generalizada simetrizável, e seja (.| .) a forma bilinear invariante padrão. Então, dada uma raiz real $\alpha$, temos $(\alpha \mid \alpha)=\left(w\left(\alpha_{i}\right) \mid w\left(\alpha_{i}\right)\right)=\left(\alpha_{i} \mid \alpha_{i}\right)$, para alguma raiz simples $\alpha_{i}$.

Definição 1.5.3. Diremos que a é uma raiz real curta (respectivamente longa) se

$$
(\alpha \mid \alpha)=\min _{i}\left(\alpha_{i} \mid \alpha_{i}\right)
$$

(respectivamente $\left.(\alpha \mid \alpha)=\max _{i}\left(\alpha_{i} \mid \alpha_{i}\right)\right)$. 
Definição 1.5.4. Uma raiz a que não é real é denominada raiz imaginária. Denotaremos por $\Delta^{i m}$ e $\Delta_{+}^{i m}$ os conjuntos de todas as raízes imaginárias e das raízes imaginárias positivas, respectivamente.

Assim, $\Delta=\Delta^{r e} \cup \Delta^{i m}$, que é uma união disjunta; e também $\Delta^{i m}=\Delta_{+}^{i m} \cup\left(-\Delta_{+}^{i m}\right)$.

Proposição 1.5.5. (a) O conjunto $\Delta_{+}^{i m}$ é $W$-invariante.

(b) Para todo $\alpha \in \Delta_{+}^{i m}$ existe uma única raiz $\beta \in-C^{V}$ (isto é, $\left\langle\beta, \alpha_{i}^{V}\right\rangle \leq 0$ para todo i), que é $W$-equivalente a $\alpha$.

(c) Se A é simetrizável e (. | .) é a forma bilinear invariante padrão, então uma raiz a é imaginária se e somente se $(\alpha \mid \alpha) \leq 0$.

(vide [14, p.61-62])

Definição 1.5.6. Para $\alpha=\sum_{i} k_{i} \alpha_{i} \in Q$, definimos o suporte de $\alpha$ (supp $\alpha$ ) como sendo o subdiagrama do diagrama de Dynkin que consiste dos vértices $i$ tais que $k_{i} \neq 0$, e de todas as arestas que ligam esses vértices.

Pelo Lema 1.6 em [14, p.11], o suporte de toda raiz $\alpha$ de uma álgebra de Lie $\mathfrak{g}(A)$ é conectado.

Teorema 1.5.7. Seja A uma matriz de Cartan generalizada indecomponivel.

(a) Se A é do tipo finito, então o conjunto $\Delta^{i m}$ é vazio.

(b) Se A é do tipo afim, então

$$
\Delta_{+}^{i m}=\{n \delta(n=1,2, \ldots)\}
$$

em que $\delta=\sum_{i=0}^{l} a_{i} \alpha_{i}$, e os $\alpha_{i}$ são as etiquetas do diagrama de Dynkin na tabela Aff.

(c) Se A é do tipo indeterminado, então existe uma raiz imaginária positiva $\alpha=\sum_{i} k_{i} \alpha_{i}$ tal que $k_{i}>0$ e $\left\langle\alpha, \alpha_{i}^{V}\right\rangle<0$, para todo $i=1, \ldots, n$.

Demonstração. Pelos resultados da seção anterior, o conjunto

$$
\left\{\alpha \in Q_{+} \mid\left\langle\alpha, \alpha_{i}^{V}\right\rangle \leq 0, i=1, \ldots, n\right\}
$$

é $\{0\}$ se $A$ é do tipo finito, é igual a $\mathbb{Z} \delta$ se $A$ é do tipo afim, e existe $\alpha=\sum_{i} k_{i} \alpha_{i}$ tal que $k_{i}>0$ e $\left\langle\alpha, \alpha_{i}^{V}\right\rangle<0$, para todo $i$, se $A$ é de tipo indeterminado. Definimos

$$
K=\left\{\alpha \in Q_{+} \backslash\{0\} \mid\left\langle\alpha, \alpha_{i}^{V}\right\rangle \leq 0 \text { para todo } i \text { e supp } \alpha \text { é conectado }\right\}
$$

e utilizamos o Teorema 5.4 em [14, p.63], que nos diz que

$$
\Delta_{+}^{i m}=\bigcup_{w \in W} w(K)
$$


Observamos que $w(\delta)=\delta$, para todo $w \in W$ e temos o resultado desejado.

Pelo Teorema 1.5.7, temos os conjuntos de raízes imaginárias e raízes imaginárias positivas de uma álgebra de Lie afim:

$$
\Delta^{i m}=\{ \pm \delta, \pm 2 \delta, \ldots\}, \Delta_{+}^{i m}=\{\delta, 2 \delta, \ldots\}
$$

Podemos então descrever os conjuntos das raízes reais $\Delta^{r e}$ e das raízes reais positivas $\Delta_{+}^{r e}$ das álgebras de Lie afim da tabela Aff 1 em termos de $\dot{\Delta}$ e $\delta$, em que $\dot{\Delta}$ é o sistema de raízes associado à álgebra de Kac-Moody $\dot{\mathfrak{g}}=\mathfrak{g}(\dot{A})$, com $\dot{A}$ obtida de $A$ pela remoção da 0 -ésima linha e coluna.

Proposição 1.5.8. (a) $\Delta^{r e}=\{\alpha+n \delta \mid \alpha \in \dot{\Delta}, n \in \mathbb{Z}\}$.

(b) $\Delta^{r e}+\delta=\Delta^{r e}$.

(c) $\Delta_{+}^{r e}=\left\{\alpha \in \Delta^{r e} \operatorname{com} n>0\right\} \cup \dot{\Delta}_{+}$.

Demonstração. Notemos que (b) e (c) seguem de (a). Para mostrar (a), utilizaremos a Proposição 5.10 em [14, p.67]. Denotemos por $a$ e $b$ os quadrados dos comprimentos de uma raiz curta e uma raiz longa, respectivamente, e sejam $\Delta_{s}^{r e}, \Delta_{l}^{r e}$ os conjuntos das raízes reais curtas e raízes reais longas.

Pelos diagramas de Dynkin da tabela Aff1, a raiz imaginária positiva minimal é sempre da forma $\delta=\alpha_{0}+a_{1} \alpha_{1}+\ldots+a_{l} \alpha_{l}$. Se $\alpha=\sum_{i=0}^{l} k_{i} \alpha_{i} \in \Delta_{s}^{r e}$, então $a=|\alpha|^{2}=\left|\alpha-k_{0} \delta\right|^{2}$, pois $\left(\alpha_{i} \mid \delta\right)=0, \forall i$ e $(\delta \mid \delta)=0$ (conforme [14, p.82]). Pela Proposição 5.10 (a), o conjunto das raízes reais curtas é

$$
\left\{\left.\alpha \in Q|| \alpha\right|^{2}=a=\min _{i}\left|\alpha_{i}\right|^{2}\right\}
$$

Logo, $\alpha-k_{0} \delta \in \dot{\Delta}_{s}$. Fazendo $\beta=\alpha-k_{0} \delta$, temos $\alpha=\beta+k_{0} \delta$, com $\beta \in \dot{\Delta}_{s}$ e $k_{0} \in \mathbb{Z}$, de onde $\Delta_{s}^{r e} \subset\left\{\alpha+n \delta \mid \alpha \in \dot{\Delta}_{s}, n \in \mathbb{Z}\right\}$.

Para a inclusão contrária, consideremos $\alpha \in \dot{\Delta}_{s}$. Então, $|\alpha|^{2}=a$, o que implica $|\alpha+r \delta|^{2}=a$, para todo $r \in \mathbb{Z}$, isto é, para todo $\alpha \in \dot{\Delta}_{s}, \alpha+r \delta \in \Delta_{s}^{r e}$, novamente pela Proposição 5.10 (a).

Seja agora $\alpha \in \dot{\Delta}_{l}$. Então, $b=|\alpha|^{2}=|\alpha+s \delta|^{2}, \forall s \in \mathbb{Z}$. Como $\alpha_{0}$ é uma raiz longa (pelos diagramas de Dynkin), usando a relação $\left(\alpha_{0} \mid \alpha_{0}\right)=a_{0}^{V} a_{0}^{-1} a_{00}=2$, temos $b=2$. Pela Proposição 5.10 (b), o conjunto de todas as raízes reais longas é exatamente

$$
\left\{\alpha=\left.\sum_{j} k_{j} \alpha_{j} \in Q|| \alpha\right|^{2}>0 \text { e } k_{j}\left|\alpha_{j}\right|^{2} /|\alpha|^{2} \in \mathbb{Z} \forall j\right\}
$$

Escrevendo $\alpha=\sum_{i=1}^{l} k_{i} \alpha_{i}$, a Proposição 5.10 (b) fornece $k_{i}\left|\alpha_{i}\right|^{2} /|\alpha|^{2} \in \mathbb{Z}$, para todo $i=1, \ldots, l$; pela mesma proposição, $\alpha+s \delta \in \Delta_{l}^{r e}$ se e somente se $s a_{i}\left|\alpha_{i}\right|^{2} /|\alpha|^{2} \in \mathbb{Z}$, para $i=1, \ldots, l$. Como $\left|\alpha_{i}\right|^{2}=2 a_{i}^{V} / a_{i}$, a condição é $\frac{2 a_{i}^{V}}{(\alpha \mid \alpha)} s \in \mathbb{Z}$, para $i=1, \ldots, l$. Como $|\alpha|^{2}=2$, a condição que precisamos satisfazer é $s a_{i}^{V} \in \mathbb{Z}$, o que naturalmente já está satisfeito, e nos dá $\alpha+s \delta \in \Delta_{l}^{r e}$.

Para a inclusão contrária, consideremos $\beta=\sum_{i=0}^{l} k_{i} \alpha_{i} \in \Delta_{l}^{r e}$. Então, $\beta-k_{0} \delta=\sum_{i=1}^{l}\left(k_{i}-k_{0} a_{i}\right) \alpha_{i}$, $\left|\beta-k_{0} \delta\right|^{2}=|\beta|^{2}=2$, e pela Proposição $5.10(\mathrm{~b}), k_{i}\left|\alpha_{i}\right|^{2} /|\beta|^{2} \in \mathbb{Z}$, para $i=0,1, \ldots, l$. Como $|\beta|^{2}=2$ 
e também $\left|\alpha_{i}\right|^{2}=2 a_{i}^{V} / a_{i}$, então $k_{0} a_{i}\left|\alpha_{i}\right|^{2} /|\beta|^{2} \in \mathbb{Z}$. Assim, pela Proposição 5.10(b), $\beta-k_{0} \delta \in \dot{\Delta}_{l}$, e $\beta=\alpha+k_{0} \delta$, para algum $\alpha \in \dot{\Delta}_{l}$ e $k_{0} \in \mathbb{Z}$.

Observação 1.5.9. Há aqui a necessidade de justificar a notação utilizada na demonstração anterior.

Se $A$ é uma matriz de Cartan generalizada do tipo afim de ordem $l+1$ (e posto $l$ ), seu diagrama de Dynkin pertence à tabela Aff1 e $a_{0}, \ldots, a_{l}$ são as etiquetas numéricas no diagrama, podemos notar que $a_{0}=1$.

Denotamos por $a_{i}^{V}(i=0, \ldots, l)$ as etiquetas do diagrama de $A^{t}$, que é obtido do diagrama original revertendo as direções das setas e mantendo a mesma enumeração dos vértices. Então, $a_{0}^{V}=1$.

Conforme resultados estudados em [14], a matriz $A$ é simetrizável e $A=\operatorname{diag}\left(a_{0} a_{0}^{V-1}, \ldots, a_{l} a_{l}^{V-1}\right) B$, em que $B=B^{t}$.

\section{6 Álgebras de Kac-Moody Afim}

Na Seção 1.1 tivemos uma ideia geral da construção das álgebras de Kac-Moody a partir de uma matriz de Cartan generalizada; já na Seção 1.4, estudamos como classificam-se essas matrizes, e consequentemente as álgebras associadas a elas. Nesta seção, estudaremos uma construção mais concreta, que será efetuada em alguns importantes passos, porém iremos manter nosso foco na construção das álgebras de Lie do tipo afim, mais especificamente aquelas associadas às matrizes da tabela Aff 1.

Seja $\mathcal{L}=\mathbb{C}\left[t, t^{-1}\right]$ a álgebra dos polinômios de Laurent na variável $t$. Um polinômio em $\mathcal{L}$ é da forma $P=\sum_{k \in \mathbb{Z}} c_{k} t^{k}$ em que todos os $c_{k}$, exceto uma quantidade finita, são nulos.

Definição 1.6.1. O resíduo de um polinômio de Laurent é definido por Res $P=c_{-1}$, e é um funcional linear em $\mathcal{L}$ caracterizado pelas propriedades:

$$
\operatorname{Res} t^{-1}=1 ; \operatorname{Res} \frac{d P}{d t}=0 .
$$

Definimos uma função bilinear $\varphi: \mathcal{L} \times \mathcal{L} \longrightarrow \mathbb{C}$ por

$$
\varphi(P, Q)=\operatorname{Res} \frac{d P}{d t} Q
$$

e são válidas as seguintes propriedades:

$$
\begin{array}{r}
\varphi(P, Q)=-\varphi(Q, P) \\
\varphi(P Q, R)+\varphi(Q R, P)+\varphi(R P, Q)=0,
\end{array}
$$

para todo $P, Q, R \in \mathcal{L}$.

De fato, como

$$
\frac{d(P Q)}{d t}=\frac{d P}{d t} Q+P \frac{d Q}{d t} \text { e Res } \frac{d P}{d t}=0, \text { para todo } P, Q \in \mathcal{L}
$$


então

$$
\begin{aligned}
0 & =\operatorname{Res} \frac{d(P Q)}{d t}=\operatorname{Res}\left(\frac{d P}{d t} Q+P \frac{d Q}{d t}\right) \\
& =\operatorname{Res} \frac{d P}{d t} Q+\operatorname{Res} P \frac{d Q}{d t}
\end{aligned}
$$

o que nos dá - Res $P \frac{d Q}{d t}=\operatorname{Res} \frac{d P}{d t} Q$, que é a igualdade desejada. Para a segunda igualdade, utilizamos a primeira:

$$
\begin{aligned}
\varphi(P Q, R) & =\operatorname{Res} \frac{d(P Q)}{d t} R=\operatorname{Res}\left(\frac{d P}{d t} Q R+\frac{d Q}{d t} P R\right) \\
& =\operatorname{Res} \frac{d P}{d t} Q R+\operatorname{Res} \frac{d Q}{d t} P R=\varphi(P, Q R)+\varphi(Q, P R)=-\varphi(Q R, P)-\varphi(P R, Q),
\end{aligned}
$$

o que nos dá $\varphi(P Q, R)+\varphi(Q R, P)+\varphi(R P, Q)=0$.

Definição 1.6.2. A álgebra de Kac-Moody afim associada a uma matriz de Cartan generalizada do tipo $X_{l}^{(1)}$ (ver Tabela Aff 1) é denominada álgebra de Kac-Moody afim não torcida. A matriz de Cartan generalizada $A$ do tipo $X_{l}^{(1)}$ (em que $X=A, B, \ldots, G$ ) é conhecida como matriz de Cartan estendida da álgebra de Lie simples de dimensão finita $\dot{\mathfrak{g}}:=\mathfrak{g}(\dot{A})$, cuja matriz de Cartan $\dot{A}$ é uma matriz do tipo finito $X_{l}$ (obtida de A removendo-se a 0-ésima coluna e a 0-ésima linha).

A partir de agora, passaremos à construção das álgebras de Kac-Moody afim não torcidas, e veremos que uma álgebra desse tipo pode ser totalmente realizada em termos de uma álgebra de Lie simples de dimensão finita $\dot{\mathfrak{g}}$ "subjacente"a ela.

Em primeiro lugar, consideremos a álgebra de loop

$$
\mathcal{L}(\dot{\mathfrak{g}}):=\mathcal{L} \otimes_{\mathbb{C}} \dot{\mathfrak{g}}
$$

$\mathcal{L}(\dot{\mathfrak{g}})$ é uma álgebra de Lie complexa de dimensão infinita com o colchete [, ]o definido por

$$
\left[t^{m} \otimes x, t^{n} \otimes y\right]_{0}=t^{m+n} \otimes[x, y](m, n \in \mathbb{Z} ; x, y \in \dot{\mathfrak{g}})
$$

e linearidade.

Em seguida, fixamos uma forma bilinear simétrica invariante não degenerada (. | .) em $\dot{\mathfrak{g}}$, a valores em $\mathbb{C}$, cuja existência é garantida pelo Teorema 1.2.3. Estendemos (. | .) a uma forma bilinear simétrica não degenerada $(. \mid \text {. })_{t}$ em $\mathcal{L}(\dot{\mathfrak{g}})$ a valores em $\mathcal{L}$ por

$$
\left(t^{m} \otimes x \mid t^{n} \otimes y\right)_{t}=t^{m+n}(x \mid y)(m, n \in \mathbb{Z} ; x, y \in \dot{\mathfrak{g}})
$$

e linearidade. 
Vejamos que $(. \mid .)_{t}$ é também invariante:

$$
\begin{aligned}
\left(\left[t^{m} \otimes x, t^{n} \otimes y\right]_{0} \mid t^{l} \otimes z\right)_{t} & =\left(t^{m+n} \otimes[x, y] \mid t^{l} \otimes z\right)_{t} \\
& =t^{(m+n)+l}([x, y] \mid z) \mathrm{e} \\
\left(t^{m} \otimes x \mid\left[t^{n} \otimes y, t^{l} \otimes z\right]_{0}\right)_{t} & =\left(t^{m} \otimes x \mid t^{n+l} \otimes[y, z]\right)_{t} \\
& =t^{m+(n+l)}(x \mid[y, z]) .
\end{aligned}
$$

Como (.| .) é invariante, temos o resultado desejado.

Estendemos também cada derivação $D$ da álgebra $\mathcal{L}$ a uma derivação da álgebra de Lie $\mathcal{L}(\dot{\mathfrak{g}})$ por

$$
D\left(t^{m} \otimes x\right)=D\left(t^{m}\right) \otimes x,(m \in \mathbb{Z} ; x \in \dot{\mathfrak{g}})
$$

e linearidade.

Definição 1.6.3. Seja $\mathfrak{g}$ uma álgebra de Lie. Um 2-cociclo sobre $\mathfrak{g}$ a valores em $\mathbb{C}$ é uma função bilinear $\psi$, a valores em $\mathbb{C}$, satisfazendo as seguintes condições:

(a) $\psi(a, b)=-\psi(b, a)$

(b) $\psi([a, b], c)+\psi([b, c], a)+\psi([c, a] b)=0,(a, b, c \in \mathfrak{g})$.

Agora então podemos definir um 2-cociclo na álgebra de Lie $\mathcal{L}(\dot{\mathfrak{g}})$ a valores em $\mathbb{C}$ por

$$
\psi(a, b)=\operatorname{Res}\left(\frac{d a}{d t} \mid b\right)_{t}(a, b \in \mathcal{L}(\dot{\mathfrak{g}})) .
$$

Veremos que, de fato, $\psi$ é um 2-cociclo.

(a) Sejam $a=t^{m} \otimes x, b=t^{n} \otimes y, \operatorname{com} m, n \in \mathbb{Z} ; x, y \in \dot{\mathfrak{g}}$. Então,

$$
\begin{aligned}
\psi\left(t^{m} \otimes x, t^{n} \otimes y\right) & =\operatorname{Res}\left(\frac{d\left(t^{m} \otimes x\right)}{d t} \mid t^{n} \otimes y\right)_{t}=\operatorname{Res}\left(\frac{d t^{m}}{d t} \otimes x \mid t^{n} \otimes y\right)_{t} \\
& =\operatorname{Res}\left(\frac{d t^{m}}{d t} t^{n}(x \mid y)\right) \\
& =(x \mid y) \operatorname{Res}\left(\frac{d t^{m}}{d t} t^{n}\right) \\
& =(x \mid y) \varphi\left(t^{m}, t^{n}\right) .
\end{aligned}
$$

Por (1.20), temos $\psi\left(t^{m} \otimes x, t^{n} \otimes y\right)=(x \mid y) \varphi\left(t^{m}, t^{n}\right)=-(x \mid y) \varphi\left(t^{n}, t^{m}\right)$. Por outro lado,

$$
\begin{aligned}
-(x \mid y) \varphi\left(t^{n}, t^{m}\right) & =-(y \mid x) \operatorname{Res}\left(\frac{d t^{n}}{d t} t^{m}\right)=-\operatorname{Res}\left(\frac{d t^{n}}{d t} t^{m}(y \mid x)\right) \\
& \left.=-\operatorname{Res}\left(\frac{d t^{n}}{d t} \otimes y \mid t^{m} \otimes x\right)\right)_{t} \\
& =-\psi\left(t^{n} \otimes y, t^{m} \otimes x\right),
\end{aligned}
$$

o que nos mostra a validade de $(a)$. 
(b) Sejam $a=t^{m} \otimes x, b=t^{n} \otimes y, c=t^{l} \otimes z, \operatorname{com} m, n, l \in \mathbb{Z} ; x, y, z \in \dot{\mathfrak{g}}$. Então,

$$
\begin{aligned}
\psi\left(\left[t^{m} \otimes x, t^{n} \otimes y\right]_{0}, t^{l} \otimes z\right) & =\psi\left(t^{m+n} \otimes[x, y], t^{l} \otimes z\right) \\
& =\operatorname{Res}\left(\frac{d\left(t^{m+n} \otimes[x, y]\right)}{d t} \mid t^{l} \otimes z\right)_{t} \\
& =\operatorname{Res}\left(\frac{d t^{m+n}}{d t} \otimes[x, y] \mid t^{l} \otimes z\right)_{t} \\
& =\operatorname{Res}\left(\frac{d t^{m+n}}{d t} t^{l}([x, y] \mid z)\right) \\
& =([x, y] \mid z) \operatorname{Res}\left(\frac{d t^{m+n}}{d t} t^{l}\right)=([x, y] \mid z) \varphi\left(t^{m+n}, t^{l}\right) .
\end{aligned}
$$

Com cálculos análogos,

$$
\begin{aligned}
\psi\left(\left[t^{n} \otimes y, t^{l} \otimes z\right]_{0}, t^{m} \otimes x\right) & =([y, z] \mid x) \varphi\left(t^{n+l}, t^{m}\right) \\
& =(x \mid[y, z]) \varphi\left(t^{n+l}, t^{m}\right)=([x, y] \mid z) \varphi\left(t^{n+l}, t^{m}\right) \mathrm{e} \\
\psi\left(\left[t^{l} \otimes z, t^{m} \otimes x\right]_{0}, t^{n} \otimes y\right) & =([z, x] \mid y) \varphi\left(t^{l+m}, t^{n}\right) \\
& =(z \mid[x, y]) \varphi\left(t^{l+m}, t^{n}\right)=([x, y] \mid z) \varphi\left(t^{l+m}, t^{n}\right) .
\end{aligned}
$$

Por $(1.21)$

$$
\begin{aligned}
& \psi\left(\left[t^{m} \otimes x, t^{n} \otimes y\right]_{0}, t^{l} \otimes z\right)+\psi\left(\left[t^{n} \otimes y, t^{l} \otimes z\right]_{0}, t^{m} \otimes x\right)+\psi\left(\left[t^{l} \otimes z, t^{m} \otimes x\right]_{0}, t^{n} \otimes y\right) \\
& =([x, y] \mid z)\left(\varphi\left(t^{m+n}, t^{l}\right)+\varphi\left(t^{n+l}, t^{m}\right)+\varphi\left(t^{l+m}, t^{n}\right)\right) \\
& =([x, y] \mid z) 0=0 .
\end{aligned}
$$

Denotemos agora por $\widetilde{\mathcal{L}}(\dot{\mathfrak{g}})$ a extensão central da álgebra de Lie $\mathcal{L}(\dot{\mathfrak{g}})$ por um centro unidimensional, associado ao cociclo $\psi$. Explicitamente, $\widetilde{\mathcal{L}}(\dot{\mathfrak{g}})=\mathcal{L}(\dot{\mathfrak{g}}) \oplus \mathbb{C} c$ (soma direta de espaços vetoriais) e o colchete é dado por

$$
[a+\lambda c, b+\mu c]=[a, b]_{0}+\psi(a, b) c(a, b \in \mathcal{L}(\dot{\mathfrak{g}}) ; \lambda, \mu \in \mathbb{C})
$$

Lema 1.6.4. $\widetilde{\mathcal{L}}(\dot{\mathfrak{g}})$ é uma álgebra de Lie com respeito ao colchete definido acima.

Demonstração. Da bilinearidade de [, ] e $\psi$, temos a bilinearidade desse novo colchete definido em $\widetilde{\mathcal{L}}(\dot{\mathfrak{g}})$. Assim, para mostrar que $\widetilde{\mathcal{L}}(\dot{\mathfrak{g}})$ é de fato uma álgebra de Lie, precisamos apenas mostrar as propriedades do colchete de Lie.

(a) O colchete é naturalmente anti simétrico, pois $[a, b]_{0}=-[b, a]_{0}$, para todo $a, b \in \mathcal{L}(\dot{\mathfrak{g}})$, e como $\psi$ é um 2- cociclo, satisfaz (1.20).

(b) Para ter $[a+\lambda c, a+\lambda c]=0$, basta notarmos que, como $\mathbb{C}$ é um corpo de característica diferente de $2, \psi(a, a)=-\psi(a, a)=0$, já que $\psi$ satisfaz (1.20), e também $[a, a]_{0}=0$, para todo $a \in \mathcal{L}(\dot{\mathfrak{g}})$. 
(c) (Identidade de Jacobi) Sejam $a+\lambda c, b+\mu c, d+\gamma c \in \widetilde{\mathcal{L}}(\dot{\mathfrak{g}})$. Então,

$$
\begin{aligned}
{[[a+\lambda c, b+\mu c], d+\gamma c] } & =\left[[a, b]_{0}+\psi(a, b) c, d+\gamma c\right] \\
& =\left[[a, b]_{0}, d\right]_{0}+\psi\left([a, b]_{0}, d\right) c \\
& =-\left[[b, d]_{0}, a\right]_{0}-\left[[d, a]_{0}, b\right]_{0}-\psi\left([b, d]_{0}, a\right) c-\psi\left([d, a]_{0}, b\right) c \\
& =-[[b+\mu c, d+\gamma c], a+\lambda c]-[[d+\gamma c, a+\lambda c], b+\mu c]
\end{aligned}
$$

de onde segue a igualdade desejada.

Definimos agora uma derivação $d$ de $\mathcal{L}(\dot{\mathfrak{g}})$ por

$$
d\left(t^{m} \otimes x\right)=m t^{m} \otimes x(m \in \mathbb{Z} ; x \in \dot{\mathfrak{g}}),
$$

e linearidade, isto é, $d=t \frac{d}{d t}$.

Lema 1.6.5. dé uma derivação de $\mathcal{L}(\dot{\mathfrak{g}})$.

Demonstração. Sejam $t^{m} \otimes x, t^{n} \otimes y, m, n \in \mathbb{Z} ; x, y \in \dot{\mathfrak{g}}$. Então,

$$
\begin{gathered}
d\left(\left[t^{m} \otimes x, t^{n} \otimes y\right]_{0}\right)=d\left(t^{m+n} \otimes[x, y]\right)=(m+n) t^{m+n}[x, y] \\
{\left[d\left(t^{m} \otimes x\right), t^{n} \otimes y\right]_{0}+\left[t^{m} \otimes x, d\left(t^{n} \otimes y\right)\right]_{0}} \\
=\left[m t^{m} \otimes x, t^{n} \otimes y\right]_{0}+\left[t^{m} \otimes x, n t^{n} \otimes y\right]_{0} \\
=m t^{m+n} \otimes[x, y]+n t^{m+n} \otimes[x, y],
\end{gathered}
$$

de onde $d$ é de fato uma derivação.

Finalmente, denotemos por $\hat{\mathcal{L}}(\dot{\mathfrak{g}})$ a álgebra de Lie que é obtida pela adjunção a $\widetilde{\mathcal{L}}(\dot{\mathfrak{g}})$ de uma derivação $d$, que age em $\mathcal{L}(\dot{\mathfrak{g}}) \operatorname{como} t \frac{d}{d t}$ e $d(c)=0$. Assim, $\hat{\mathcal{L}}(\dot{\mathfrak{g}})$ é um espaço vetorial sobre $\mathbb{C}$

$$
\hat{\mathcal{L}}(\dot{\mathfrak{g}})=\mathcal{L}(\dot{g}) \otimes \mathbb{C} c \otimes \mathbb{C} d
$$

com o colchete definido por

$$
\begin{aligned}
& {\left[t^{m} \otimes x+\lambda c+\mu d, t^{n} \otimes y+\lambda_{1} c+\mu_{1} d\right]} \\
& =\left(t^{m+n} \otimes[x, y]+\mu n t^{n} \otimes y-\mu_{1} m t^{m} \otimes x\right)+m \delta_{m,-n}(x \mid y) c, \quad\left(x, y \in \dot{\mathfrak{g}}, \lambda, \lambda_{1}, \mu, \mu_{1} \in \mathbb{C}\right) .
\end{aligned}
$$

Antes de mostrarmos que $\hat{\mathcal{L}}(\dot{\mathfrak{g}})$ é de fato uma álgebra de Kac-Moody afim associada à matriz $A$ do tipo $X_{l}^{(1)}$, iremos mostrar que $d$ é uma derivação da álgebra de Lie $\widetilde{\mathcal{L}}(\dot{\mathfrak{g}})$. Faremos isso de forma mais geral. Denotemos por $d_{s}$ o endomorfismo do espaço vetorial $\widetilde{\mathcal{L}}(\dot{\mathfrak{g}})$ definido por

$$
d_{s} \uparrow_{\mathcal{L}(\dot{g})}=-t^{s+1} \frac{d}{d t} ; d_{s}(c)=0 .
$$


Em particular, $d_{0}=-d$.

Proposição 1.6.6. $d_{s}$ é uma derivação em $\widetilde{\mathcal{L}}(\dot{\mathfrak{g}})$.

Demonstração. Seja $D:=d_{s}$; claramente, $D$ é uma derivação em $\mathcal{L}$, e podemos estendê-la a uma derivação em $\mathcal{L}(\dot{\mathfrak{g}})$. Então, como $D$ é uma derivação em $\mathcal{L}(\dot{\mathfrak{g}})$, para todo $a, b \in \mathcal{L}(\dot{g})$ e $\lambda, \mu \in \mathbb{C}$, temos:

$$
D([a+\lambda c, b+\mu c])=D\left([a, b]_{0}\right)+D(\psi(a, b) c)=D\left([a, b]_{0}\right)=[D(a), b]_{0}+[a, D(b)]_{0},
$$

e queremos mostrar que

$$
D([a+\lambda c, b+\mu c])=[D(a+\lambda c), b+\mu c]+[a+\lambda c, D(b+\mu c)]=[a, D(b)]+[D(a), b] .
$$

Por outro lado, temos as igualdades:

$$
\begin{aligned}
& {[D(a), b]=[D(a), b]_{0}+\psi(D(a), b) c} \\
& {[a, D(b)]=[a, D(b)]_{0}+\psi(a, D(b)) c .}
\end{aligned}
$$

Para obter o resultado desejado, precisamos então verificar que

$$
\psi(D(a), b)+\psi(a, D(b))=0 .
$$

Sejam agora $a=P \otimes x, b=Q \otimes y(P, Q \in \mathcal{L} ; x, y \in \dot{\mathfrak{g}})$; então, o lado esquerdo da equação (1.24) é:

$$
\begin{aligned}
& \psi(D(P \otimes x), Q \otimes y)+\psi(P \otimes x, D(Q \otimes y)) \\
& =\psi(D(P) \otimes x, Q \otimes y)+\psi(P \otimes x, D(Q) \otimes y) \\
& =\operatorname{Res}\left(\frac{d D(P)}{d t} \otimes x \mid Q \otimes y\right)_{t}+\operatorname{Res}\left(\frac{d P}{d t} \otimes x \mid D(Q) \otimes y\right)_{t} \\
& =\operatorname{Res}\left(\frac{d D(P)}{d t} Q(x \mid y)\right)+\operatorname{Res}\left(\frac{d P}{d t} D(Q)(x \mid y)\right) \\
& =(x \mid y)(\varphi(D(P), Q)+\varphi(P, D(Q)) \\
& =(x \mid y)(-\varphi(Q, D(P))+\varphi(P, D(Q))) \\
& =(x \mid y) \operatorname{Res}\left(-\frac{d Q}{d t} t^{s+1} \frac{d P}{d t}+\frac{d P}{d t} t^{s+1} \frac{d Q}{d t}\right)=0
\end{aligned}
$$

fornecendo o resultado desejado.

Mostraremos agora que $\hat{\mathcal{L}}(\dot{g})=\mathcal{L}(\dot{\mathfrak{g}}) \otimes \mathbb{C} c \otimes \mathbb{C} d$ é uma álgebra de Kac-Moody afim associada à matriz $A$ do tipo $X_{l}^{(1)}$.

Sejam $\dot{\mathfrak{h}}$ a subálgebra de Cartan de $\dot{\mathfrak{g}}$, e $\dot{\Delta} \subset \dot{\mathfrak{h}}^{*}$ o sistema de raízes de $\dot{\mathfrak{g}}$. Consideremos também $\left\{\alpha_{1}, \ldots, \alpha_{l}\right\}$ a base de raízes, e $\left\{H_{1}, \ldots, H_{l}\right\}$ a base de co-raízes; $E_{i}, F_{i}(i=1, \ldots, l)$ os geradores de Chevalley. Seja $\theta=\sum_{i=1}^{l} a_{1} \alpha_{i}$ a raiz de altura máxima do sistema de raízes $\dot{\Delta}$, e $\dot{\mathfrak{g}}=\underset{\alpha \in \dot{\Delta} \cup\{0\}}{\bigoplus} \dot{\mathfrak{g}}_{\alpha}$ a decomposição em espaços de raízes de $\dot{\mathfrak{g}}$. Conforme Proposição 1.5.2, $(\alpha \mid \alpha) \neq 0$ e $\operatorname{dim} \dot{\mathfrak{g}}_{\alpha}=1$, 
para todo $\alpha \in \dot{\Delta}$, já que não existem raízes imaginárias no sistema de raízes de uma álgebra de Kac-Moody do tipo finito. Seja também $\dot{\omega}$ a involução de Chevalley de $\dot{\mathfrak{g}}$.

Escolhemos $F_{0} \in \dot{g}_{\theta}$ tal que $\left(F_{0} \mid \dot{\omega}\left(F_{0}\right)\right)=-2 /(\theta \mid \theta)$ e definimos $E_{0}=\dot{\omega}\left(F_{0}\right)$. Assim, definindo $\theta^{V}=\frac{2}{(\theta \mid \theta)} \nu^{-1}(\theta)$, pelo Teorema 1.2.3(e), temos:

$$
\left[E_{0}, F_{0}\right]=\left(E_{0} \mid F_{0}\right) \nu^{-1}(\theta)=-\frac{2}{(\theta \mid \theta)} \nu^{-1}(\theta)=-\theta^{V}
$$

pois $\dot{\omega}\left(E_{0}\right) \in \dot{\mathfrak{g}}_{-\theta}$.

Os elementos $E_{i}(i=0,1, \ldots, l)$ geram a álgebra de Lie $\dot{\mathfrak{g}}$. De fato, como $\dot{\mathfrak{g}}$ é simples, então sob a ação da representação adjunta dos elementos de $\mathfrak{U}(\dot{\mathfrak{g}})$ em $E_{0}, \dot{\mathfrak{g}}=\mathfrak{U}(\dot{\mathfrak{g}}) \cdot E_{0}$.

Voltando a $\hat{\mathcal{L}}(\dot{\mathfrak{g}})$, temos que $\mathbb{C} c$ é seu centro de dimensão 1 . Observemos ainda que $1 \otimes \dot{\mathfrak{g}}$ é uma subálgebra de $\hat{\mathcal{L}}(\dot{\mathfrak{g}})$, com a qual $\dot{\mathfrak{g}}$ pode ser identificada fazendo $x \longmapsto 1 \otimes x$, para todo $x \in \dot{\mathfrak{g}}$.

Além disso,

$$
\mathfrak{h}:=\dot{\mathfrak{h}}+\mathbb{C} c+\mathbb{C} d
$$

é uma subálgebra comutativa de $\hat{\mathcal{L}}(\dot{\mathfrak{g}})$, de dimensão $l+2$. Estendemos $\lambda \in \dot{\mathfrak{h}}^{*}$ a um funcional linear sobre $\mathfrak{h}$ definindo $\langle\lambda, c\rangle=\langle\lambda, d\rangle=0$, de modo a identificar $\dot{\mathfrak{h}}^{*}$ como um subespaço de $\mathfrak{h}^{*}$. Denotamos por $\delta$ o funcional linear sobre $\mathfrak{h}$ definido por $\delta_{\uparrow_{\mathfrak{h}+\mathbb{C} c}}=0,\langle\delta, d\rangle=1$. Sejam agora

$$
\begin{array}{r}
e_{0}=t \otimes E_{0}, f_{0}=t^{-1} \otimes F_{0}, \\
e_{i}=1 \otimes E_{i}, f_{i}=1 \otimes F_{i},
\end{array}
$$

para todo $i=1, \ldots, l$. Por $(1.25)$, temos:

$$
\left[e_{0}, f_{0}\right]=\left[t \otimes E_{0}, t^{-1} \otimes F_{0}\right]=1 \otimes\left[E_{0}, F_{0}\right]+\left(E_{0} \mid F_{0}\right) c=\frac{2}{(\theta \mid \theta)} c-\theta^{V}
$$

Descreveremos em seguida o sistema de raízes e a decomposição em espaços de raízes de $\hat{\mathcal{L}}(\dot{\mathfrak{g}})$ com respeito a $\mathfrak{h}$ :

$$
\begin{gathered}
\Delta=\{j \delta+\gamma, \text { em que } j \in \mathbb{Z}, \gamma \in \dot{\Delta}\} \cup\{j \delta, \text { em que } j \in \mathbb{Z} \backslash\{0\}\}, \\
\hat{\mathcal{L}}(\dot{\mathfrak{g}})=\mathfrak{h} \oplus\left(\bigoplus_{\alpha \in \Delta} \mathcal{L}(\dot{\mathfrak{g}})_{\alpha}\right), \text { em que } \\
\mathcal{L}(\dot{\mathfrak{g}})_{j \delta+\gamma}=t^{j} \otimes \dot{\mathfrak{g}}_{\gamma}, \mathcal{L}(\dot{\mathfrak{g}})_{j \delta}=t^{j} \otimes \dot{\mathfrak{h}} .
\end{gathered}
$$

Definimos também:

$$
\begin{aligned}
\Pi & =\left\{\alpha_{0}:=\delta-\theta, \alpha_{1}, \ldots, \alpha_{l}\right\}, \\
\Pi^{V} & =\left\{\alpha_{0}^{V}:=\frac{2}{(\theta \mid \theta)} c-1 \otimes \theta^{V}, \alpha_{1}^{V}:=1 \otimes H_{1}, \ldots, \alpha_{l}^{V}:=1 \otimes H_{l}\right\} .
\end{aligned}
$$


Na seção 6.4 de [14] foi introduzido o elemento

$$
\theta=\delta-a_{0} \alpha_{0}=\sum_{i=1}^{l} a_{i} \alpha_{i} \in \dot{Q}
$$

que é exatamente o mesmo $\theta$ que estamos definindo agora. Assim, pela Proposição 6.4(a) em [14, p.85],

$$
A=\left(\left\langle\alpha_{j}, \alpha_{i}^{V}\right\rangle\right)_{i, j=0}^{l}
$$

Portanto, $\left(\mathfrak{h}, \Pi, \Pi^{V}\right)$ é uma realização da matriz afim $A$ com a qual iniciamos a construção. Na verdade, $\Pi$ e $\Pi^{V}$ são linearmente independentes e $2 n-$ posto $A=2(l+1)-l=l+2=\operatorname{dim} \mathfrak{h}$.

Teorema 1.6.7. Seja $\dot{\mathfrak{g}}$ uma álgebra de Lie simples complexa de dimensão finita, e seja A sua matriz de Cartan estendida. Então, $\hat{\mathcal{L}}(\dot{\mathfrak{g}})$ é a álgebra de Kac-Moody afim associada à matriz afim $A, \mathfrak{h}$ é sua subálgebra de Cartan, $\Pi e \Pi^{V}$ são as bases de raízes e co-raízes, e $e_{0}, \ldots, e_{l}, f_{0}, \ldots, f_{l}$ são os geradores de Chevalley.

(vide [14, p.101].

Corolário 1.6.8. Seja $\mathfrak{g}(A)$ uma álgebra de Lie afim não torcida de posto $l+1$. Então, a multiplicidade de cada raiz imaginária de $\mathfrak{g}(A)$ é $l$.

Observação 1.6.9. Dada uma álgebra de Lie simples de dimensão finita $\mathfrak{g}$, a álgebra de Lie $\hat{\mathcal{L}}(\mathfrak{g})$ é usualmente referenciada na literatura por álgebra afim associada a $\mathfrak{g}$ ou afinização de $\mathfrak{g}$.

Podemos ainda descrever o restante das noções clássicas para álgebras de Kac-Moody afim.

Em primeiro lugar, na seção 6.2 de [14], foi introduzida uma forma invariante normalizada, que pode ser descrita como segue. Tomando a forma invariante normalizada (. . .) em $\dot{\mathfrak{g}}$ e estendendo para toda a álgebra $\hat{\mathcal{L}}(\dot{\mathfrak{g}})$ por

$$
\begin{aligned}
(P \otimes x \mid Q \otimes y) & =\left(\text { Res } t^{-1} P Q\right)(x \mid y), \quad(x, y \in \dot{\mathfrak{g}}, P, Q \in \mathcal{L}) \\
(\mathbb{C} c+\mathbb{C} d \mid \mathcal{L}(\dot{\mathfrak{g}})) & =0 ;(c \mid c)=(d \mid d)=0 ;(c \mid d)=1 .
\end{aligned}
$$

Seja agora $\dot{\mathfrak{g}}=\dot{\mathfrak{n}}_{-} \oplus \dot{\mathfrak{h}} \oplus \dot{\mathfrak{n}}_{+}$a decomposição triangular de $\dot{\mathfrak{g}}$. Então, a decomposição triangular de $\hat{\mathcal{L}}(\dot{\mathfrak{g}})$ pode ser expressa como segue:

$$
\begin{aligned}
\hat{\mathcal{L}}(\dot{\mathfrak{g}}) & =\mathfrak{n}_{-} \oplus \mathfrak{h} \oplus \mathfrak{n}_{+}, \text {em que } \\
\mathfrak{n}_{-} & =\left(t^{-1} \mathbb{C}\left[t^{-1}\right] \otimes\left(\dot{\mathfrak{n}}_{+}+\dot{\mathfrak{h}}\right)\right)+\mathbb{C}\left[t^{-1}\right] \otimes \dot{\mathfrak{n}}_{-} \\
\mathfrak{n}_{+} & =\left(t \mathbb{C}[t] \otimes\left(\dot{\mathfrak{n}}_{-}+\dot{\mathfrak{h}}\right)\right)+\mathbb{C}[t] \otimes \dot{\mathfrak{n}}_{+} .
\end{aligned}
$$

Finalmente, expressamos a involução de Chevalley $\omega$ de $\hat{\mathcal{L}}(\dot{\mathfrak{g}})$ em termos da involução de Chevalley $\dot{\omega}$ de $\dot{\mathfrak{g}}$ : 


$$
\omega(P(t) \otimes x+\lambda c+\mu d)=P\left(t^{-1}\right) \otimes \dot{\omega}(x)-\lambda c-\mu d,
$$

em que $P(t) \in \mathcal{L}, x \in \dot{\mathfrak{g}} ; \lambda, \mu \in \mathbb{C}$. Na verdade, temos $\omega\left(e_{i}\right)=-f_{i}, \omega\left(f_{i}\right)=-e_{i}(i=1, \ldots, l)$ e $\omega_{\mathfrak{l}}=-I d$. Além disso,

$$
\omega\left(e_{0}\right)=\omega\left(t \otimes E_{0}\right)=t^{-1} \otimes \dot{\omega}\left(E_{0}\right)=-t^{-1} \otimes F_{0}=-f_{0},
$$

e similarmente $\omega\left(f_{0}\right)=-e_{0}$.

Um exemplo de álgebra de Kac-Moody afim é a álgebra objeto de estudo deste trabalho, denotada por $\hat{\mathfrak{s l}}(2, \mathbb{C})$ :

Exemplo 1.6.10. A álgebra de Kac-Moody afim que denotamos por $\hat{\mathfrak{s l}}(2, \mathbb{C})$ é a afinização da álgebra de Lie das matrizes $2 \times 2$ de traço zero $\dot{\mathfrak{g}}=\mathfrak{s l}(2, \mathbb{C})$ :

$$
\hat{\mathfrak{s l}}(2, \mathbb{C})=\left(\mathfrak{s l}(2, \mathbb{C}) \otimes \mathbb{C}\left[t, t^{-1}\right]\right) \oplus \mathbb{C} c \oplus \mathbb{C} d,
$$

com colchete dado por :

$$
\left[a \otimes t^{m}, b \otimes t^{n}\right]=[a, b] \otimes t^{m+n}+\delta_{m,-n}(a \mid b) c,\left[d, a \otimes t^{m}\right]=m a \otimes t^{m},[c, \mathfrak{g}]=0,
$$

para todo $a, b \in \dot{\mathfrak{g}}, m, n \in \mathbb{Z}$, e a forma bilinear (.| .) é a forma de Killing de $\dot{\mathfrak{g}}$, definida por $(a \mid b)=\operatorname{tr}(a b)$, para todo $a, b \in \dot{\mathfrak{g}}$.

Temos ainda que $\dot{\mathfrak{g}}$ é a álgebra de Lie simples associada à matriz $A_{1}=(2)$, e $\hat{\mathfrak{s} l}(2, \mathbb{C})$ está associada à matriz $A_{1}^{(1)}=\left(\begin{array}{cc}2 & -2 \\ -2 & 2\end{array}\right)$. 


\section{Capítulo 2}

\section{Módulos de Verma}

Este capítulo tem por objetivo o estudo dos módulos de Verma sobre álgebras de Lie com decomposição triangular. Em particular, para as álgebras de Kac-Moody afim, estudaremos a construção dos módulos de Verma imaginários (IVM) e J-imaginários.

\subsection{Produto Tensorial}

Iniciamos o capítulo com algumas considerações a respeito do produto tensorial de módulos sobre álgebras não comutativas, tendo em vista a sua utilização na construção dos módulos de Verma, dos módulos de Verma imaginários, e dos J-imaginários.

Ao longo desta seção $\mathcal{A}$ denotará uma álgebra associativa, não necessariamente comutativa sobre $\mathbb{C}$. Destacamos que é suficiente considerar álgebras associativas, pois estamos interessados no produto tensorial de módulos sobre álgebras envolventes universais.

Definição 2.1.1. Sejam $\mathcal{A}$ uma álgebra, $M_{\mathcal{A}}$ um $\mathcal{A}$-módulo à direita e ${ }_{\mathcal{A}} N$ um $\mathcal{A}$-módulo à esquerda, e seja $G$ um grupo abeliano (aditivo). Uma função $f: M \times N \longrightarrow G$ é chamada de $\mathcal{A}$-biaditiva se, para todo $m, m^{\prime} \in M, n, n^{\prime} \in N$ e $a \in \mathcal{A}$,

$$
\begin{aligned}
f\left(m+m^{\prime}, n\right) & =f(m, n)+f\left(m^{\prime}, n\right), \\
f\left(m, n+n^{\prime}\right) & =f(m, n)+f\left(m, n^{\prime}\right) e \\
f(m a, n) & =f(m, a n) .
\end{aligned}
$$

Definição 2.1.2. Dada uma álgebra $\mathcal{A}$ e módulos $M_{\mathcal{A}}$ e ${ }_{\mathcal{A}} N$, o produto tensorial de $M$ e $N$ é um $\operatorname{par}\left(M \otimes_{\mathcal{A}} N, \otimes\right)$, em que $M \otimes_{\mathcal{A}} N$ é um grupo abeliano, e $\otimes$ é uma função $\mathcal{A}$-biaditiva

$$
\otimes: M \times N \longrightarrow M \otimes_{\mathcal{A}} N
$$

tal que, para cada grupo abeliano $G$ e cada função $\mathcal{A}$-biaditiva $f: M \times N \longrightarrow G$, existe um único homomorfismo de $\mathbb{Z}$-módulos $g: M \otimes_{\mathcal{A}} N \longrightarrow G$ que torna o diagrama abaixo comutativo 


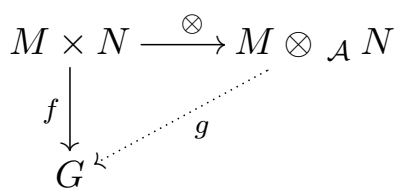

Observação 2.1.3. Notemos que, se um produto tensorial de $M$ e $N$ existe, então ele é único a menos de isomorfismo.

De fato, se $\left(\left(M \otimes_{\mathcal{A}} N\right)_{1}, \otimes_{1}\right)$ e $\left(\left(M \otimes_{\mathcal{A}} N\right)_{2}, \otimes_{2}\right)$ são produtos tensoriais de $M$ e $N$, então existem únicos homomorfismos de $\mathbb{Z}$-módulos

$$
g_{1}:\left(M \otimes_{\mathcal{A}} N\right)_{1} \longrightarrow\left(M \otimes_{\mathcal{A}} N\right)_{2}
$$

tal que $g_{1} \circ \otimes_{1}=\otimes_{2}$ e

$$
g_{2}:\left(M \otimes_{\mathcal{A}} N\right)_{2} \longrightarrow\left(M \otimes_{\mathcal{A}} N\right)_{1}
$$

tal que $g_{2} \circ \otimes_{2}=\otimes_{1}$, pela definição de produto tensorial.

Portanto, para todo $m \in M$ e $n \in N$, é fácil ver que $g_{1} \circ g_{2}:\left(M \otimes_{\mathcal{A}} N\right)_{2} \longrightarrow\left(M \otimes_{\mathcal{A}} N\right)_{2}$ é tal que $g_{1}\left(g_{2}\left(m \otimes_{2} n\right)\right)=m \otimes_{2} n$, e $g_{2} \circ g_{1}:\left(M \otimes_{\mathcal{A}} N\right)_{1} \longrightarrow\left(M \otimes_{\mathcal{A}} N\right)_{1}$ é tal que $g_{2}\left(g_{1}\left(m \otimes_{1} n\right)\right)=m \otimes_{1} n$. Porém, ainda pela definição e propriedades do produto tensorial, $I d_{2}:\left(M \otimes_{\mathcal{A}} N\right)_{2} \longrightarrow\left(M \otimes_{\mathcal{A}} N\right)_{2}$ é o único $\mathbb{Z}$-homomorfismo tal que $I d_{2}\left(m \otimes_{2} n\right)=m \otimes_{2} n$, o que nos dá $I d_{2}=g_{1} \circ g_{2}$. Analogamente, $I d_{1}=g_{2} \circ g_{1}$. Concluímos que $g_{1}$ e $g_{2}$ são $\mathbb{Z}$-homomorfismos inversos um do outro, mostrando que $\left(M \otimes_{\mathcal{A}} N\right)_{1} \cong\left(M \otimes_{\mathcal{A}} N\right)_{2}$

Proposição 2.1.4. Se $\mathcal{A}$ é uma álgebra e $M_{\mathcal{A}}$ e ${ }_{\mathcal{A}} N$ são módulos, então existe o produto tensorial de $M$ e $N$.

(vide [19, p. 576-577]).

No caso em que $\mathcal{A}$ é uma álgebra comutativa, $M \otimes_{\mathcal{A}} N$ tem uma estrutura de módulo sobre $\mathcal{A}$, o que não ocorre no caso não comutativo. Veremos agora que, sob algumas condições, pode-se obter uma estrutura de módulo para o grupo abeliano $M \otimes_{\mathcal{A}} N$, ainda que não seja sobre $\mathcal{A}$.

Definição 2.1.5. Sejam $\mathcal{A}$ e $\mathcal{B}$ álgebras, e seja $M$ um grupo abeliano. Então, diremos que $M$ é um $(\mathcal{A}, \mathcal{B})$-bimódulo, denotado por ${ }_{\mathcal{A}} M_{\mathcal{B}}$, se $M$ é um $\mathcal{A}$-módulo à esquerda e um $\mathcal{B}$-módulo à direita, $e$ as duas multiplicações por escalar estão relacionadas por uma lei associativa:

$$
a(m b)=(a m) b,
$$

para todo $a \in \mathcal{A}, b \in \mathcal{B}$ e $m \in M$.

Se $M$ é um $(\mathcal{A}, \mathcal{B})$-bimódulo, é permitido escrever $a m b$ sem os parênteses.

Lema 2.1.6. Sejam $\mathcal{A}$ e $\mathcal{B}$ álgebras, ${ }_{\mathcal{A}} M_{\mathcal{B}}$ um $(\mathcal{A}, \mathcal{B})$-bimódulo e consideremos ${ }_{\mathcal{B}} N$. Então, o produto tensorial $M \otimes_{\mathcal{B}} N$ é um $\mathcal{A}$-módulo à esquerda, em que $a(m \otimes n)=(a m) \otimes n$, para todo $a \in \mathcal{A}$, $m \in M$ e $n \in N$. Similarmente, dados $M_{\mathcal{B}}$ e ${ }_{\mathcal{B}} N_{\mathcal{A}}$, o produto tensorial $M \otimes_{\mathcal{B}} N$ é um $\mathcal{A}$-módulo à direita, em que $(m \otimes n) a=m \otimes(n a)$, para todo $a \in \mathcal{A}, m \in M$ e $n \in N$. 
Demonstração. Faremos a demonstração da segunda afirmação.

Pela definição de produto tensorial, $M \otimes_{\mathcal{B}} N$ é um grupo abeliano. Precisamos então verificar que $M \otimes_{\mathcal{B}} N$ é um espaço vetorial sobre $\mathbb{C}$, e que os axiomas de módulo estão satisfeitos.

Para cada $a \in \mathcal{A}$ fixado, vamos considerar a função

$$
\begin{aligned}
f_{a}: M \times N & \longrightarrow M \otimes_{\mathcal{B}} N \\
(m, n) & \longmapsto m \otimes(n a),
\end{aligned}
$$

para todo $m \in M$ e $n \in N$, e mostrar que $f_{a}$ é uma função $\mathcal{B}$-biaditiva. De fato,

$$
\begin{aligned}
f_{a}\left(m_{1}+m_{2}, n\right) & =\left(m_{1}+m_{2}\right) \otimes(n a)=m_{1} \otimes(n a)+m_{2} \otimes(n a)=f_{a}\left(m_{1}, n\right)+f_{a}\left(m_{2}, n\right) ; \\
f_{a}\left(m, n_{1}+n_{2}\right) & =m \otimes\left(\left(n_{1}+n_{2}\right) a\right)=m \otimes\left(n_{1} a+n_{2} a\right)=m \otimes\left(n_{1} a\right)+m \otimes\left(n_{2} a\right) \\
& =f_{a}\left(m, n_{1}\right)+f_{a}\left(m, n_{2}\right) ; \\
f_{a}(m b, n) & =(m b) \otimes(n a)=m \otimes(b(n a))=m \otimes((b n) a)=f_{a}(m, b n),
\end{aligned}
$$

para todo $m, m_{1}, m_{2} \in M, n, n_{1}, n_{2} \in N$ e $b \in \mathcal{B}$. Pela definição e propridades do produto tensorial de $M$ e $N$, existe um único homomorfismo de $\mathbb{Z}$-módulos $\phi_{a}: M \otimes_{\mathcal{B}} N \longrightarrow M \otimes_{\mathcal{B}} N$ tal que $\phi_{a} \circ \otimes=f_{a}$, isto é, $\phi_{a}(m \otimes n)=f_{a}(m, n)=m \otimes(n a)$, para todo $m \in M, n \in N$.

Definimos então a multiplicação à direita por um elemento de $\mathcal{A}$ da seguinte forma:

$$
x a:=\phi_{a}(x)
$$

para todo $x \in M \otimes_{\mathcal{B}} N$ e todo $a \in A$. Em particular, para todo $m \in M$ e $n \in N$, teremos $(m \otimes n) a=\phi_{a}(m \otimes n)=m \otimes(n a)$. Como todo elemento $x \in M \otimes_{\mathcal{B}} N$ é da forma $x=\sum_{i=1}^{k}\left(m_{i} \otimes n_{i}\right)$, com $m_{i} \in M$ e $n_{i} \in N$, para todo $i \in\{1, \ldots, k\}$, temos:

$$
x a=\phi_{a}(x)=\phi_{a}\left(\sum_{i=1}^{k}\left(m_{i} \otimes n_{i}\right)\right)=\sum_{i=1}^{k}\left(\phi_{a}\left(m_{i} \otimes n_{i}\right)\right)=\sum_{i=1}^{k}\left(m_{i} \otimes\left(n_{i} a\right)\right) .
$$

$M \otimes_{\mathcal{B}} N$ é de fato um $\mathcal{A}$-módulo à direita:

(a) Se $\mathcal{A}$ é uma álgebra com unidade 1 , então

$$
\left(\sum_{i=1}^{k}\left(m_{i} \otimes n_{i}\right)\right) 1={ }^{2.1} \sum_{i=1}^{k}\left(m_{i} \otimes\left(n_{i} 1\right)\right)=\sum_{i=1}^{k}\left(m_{i} \otimes n_{i}\right) .
$$


(b) Como $\phi_{a}$ é um homomorfismo de grupos abelianos, para todo $a \in \mathcal{A}$, então

$$
\left(\sum_{i=1}^{k}\left(m_{i} \otimes n_{i}\right)+\sum_{j=1}^{l}\left(m_{j} \otimes n_{j}\right)\right) a=\left(\sum_{i=1}^{k}\left(m_{i} \otimes n_{i}\right)\right) a+\left(\sum_{j=1}^{l}\left(m_{j} \otimes n_{j}\right)\right) a .
$$

(c) Para $a, b \in \mathcal{A}$,

$$
\begin{aligned}
\left(\sum_{i=1}^{k}\left(m_{i} \otimes n_{i}\right)\right)(a+b) & ={ }^{2.1} \sum_{i=1}^{k}\left(m_{i} \otimes\left(n_{i}(a+b)\right)\right)=\sum_{i=1}^{k}\left(m_{i} \otimes\left(n_{i} a+n_{i} b\right)\right) \\
& =\sum_{i=1}^{k}\left(\left(m_{i} \otimes\left(n_{i} a\right)\right)+\left(m_{i} \otimes\left(n_{i} b\right)\right)\right)=\sum_{i=1}^{k}\left(m_{i} \otimes\left(n_{i} a\right)\right)+\sum_{i=1}^{k}\left(m_{i} \otimes\left(n_{i} b\right)\right) \\
& =2.1\left(\sum_{i=1}^{k}\left(m_{i} \otimes n_{i}\right)\right) a+\left(\sum_{i=1}^{k}\left(m_{i} \otimes n_{i}\right)\right) b .
\end{aligned}
$$

(d) Para $a, b \in \mathcal{A}$,

$$
\begin{aligned}
\left(\sum_{i=1}^{k}\left(m_{i} \otimes n_{i}\right)\right)(a b) & ={ }^{2.1} \sum_{i=1}^{k}\left(m_{i} \otimes\left(n_{i}(a b)\right)\right)=\sum_{i=1}^{k}\left(m_{i} \otimes\left(\left(n_{i} a\right) b\right)\right) \\
& =\left(\sum_{i=1}^{k}\left(m_{i} \otimes\left(n_{i} a\right)\right)\right) b=\left(\left(\sum_{i=1}^{k}\left(m_{i} \otimes n_{i}\right)\right) a\right) b .
\end{aligned}
$$

(e) Para cada $\alpha \in \mathbb{C}$, definimos a função

$$
\begin{aligned}
g_{\alpha}: M \times N & \longrightarrow M \otimes_{\mathcal{B}} N \\
(m, n) & \longmapsto m \otimes(\alpha n),
\end{aligned}
$$

para todo $m \in M, n \in N$, e com cálculos análogos aos efetuados na demonstração de que $f_{a}$ é uma função $\mathcal{B}$-biaditiva, é possível mostrar que $g_{\alpha}$ é também uma função $\mathcal{B}$-biaditiva, observando que, pelas propriedades de módulo sobre uma álgebra, $\alpha(b n)=(\alpha b) n=b(\alpha n)$ para todo $n \in N, b \in \mathcal{B}$ e $\alpha \in \mathbb{C}$ e então

$$
g_{\alpha}(m b, n)=(m b) \otimes(\alpha n)=m \otimes(b(\alpha n))=m \otimes(\alpha(b n))=g_{\alpha}(m, b n) .
$$

De modo análogo ao da verificação dos axiomas de módulo que já foram mostrados até aqui, verifica-se que $M \otimes_{\mathcal{B}} N$ é um espaço vetorial sobre $\mathbb{C}$, com a multiplicação por escalar definida 
por

$$
\alpha y:=\eta_{\alpha}(y)
$$

para todo $y \in M \otimes_{\mathcal{B}} N$, em que $\eta_{\alpha}: M \otimes_{\mathcal{B}} N \longrightarrow M \otimes_{\mathcal{B}} N$ é o único $\mathbb{Z}$-homomorfismo tal que $\eta_{\alpha} \circ \otimes=g_{\alpha}$. Finalmente, verificaremos o último axioma de módulo, como segue:

(f) Para $a \in \mathcal{A}, \alpha \in \mathbb{C}$,

$$
\begin{aligned}
\left(\sum_{i=1}^{k}\left(m_{i} \otimes n_{i}\right)\right)(\alpha a) & ={ }^{2.1} \sum_{i=1}^{k}\left(m_{i} \otimes\left(n_{i}(\alpha a)\right)\right)=\sum_{i=1}^{k}\left(m_{i} \otimes\left(\alpha n_{i}\right) a\right) \\
& =\left(\sum_{i=1}^{k}\left(m_{i} \otimes\left(\alpha n_{i}\right)\right)\right) a=\left(\alpha\left(\sum_{i=1}^{k}\left(m_{i} \otimes n_{i}\right)\right)\right) a \\
& =\alpha\left(\left(\sum_{i=1}^{k}\left(m_{i} \otimes n_{i}\right)\right) a\right) .
\end{aligned}
$$

Observação 2.1.7. Notemos que, no caso particular em que $\mathcal{B}$ é uma subálgebra de $\mathcal{A}, \mathcal{A} \otimes_{\mathcal{B}} W$ é um $\mathcal{A}$-módulo à esquerda, para qualquer $\mathcal{B}$-módulo $W$ à esquerda.

\subsection{Decomposição Triangular}

Tendo como base a ideia de que as álgebras de $\operatorname{Lie} \mathfrak{g l}(n, \mathbb{C}), n \in \mathbb{N}$, podem ser naturalmente decompostas em uma soma direta de três subálgebras: a álgebra das matrizes triangulares estritamente superiores, a álgebra das matrizes triangulares estritamente inferiores e a álgebra das matrizes diagonais, surge o conceito de decomposição triangular de uma álgebra de Lie qualquer. Há uma certa quantidade de resultados e construções que podem ser feitos utilizando o fato de que uma álgebra de Lie possui essa decomposição, incluindo a obtenção dos módulos de Verma.

Nesta seção, estudaremos a decomposição triangular de álgebras de Lie sobre $\mathbb{C}$.

Definição 2.2.1. Sejam $\mathfrak{h}$ uma álgebra de Lie abeliana, $\pi: \mathfrak{h} \longrightarrow \mathfrak{g l}(V)$ uma representação de $\mathfrak{h}$ em um $\mathbb{C}$-espaço vetorial $V$, e $\phi \in \mathfrak{h}^{*}$. Um vetor não nulo $v \in V$ é chamado de vetor de peso com peso $\phi$ (relativo a $\mathfrak{h}$ e $\pi$ ) se para todo $h \in \mathfrak{h}$

$$
\pi(h) v=\phi(h) v .
$$

Para cada $\phi \in \mathfrak{h}^{*}$, definimos o $\phi$-espaço de peso $V_{\phi}$ por

$$
V_{\phi}=\{v \in V \mid \pi(h) v=\phi(h) v, \text { para todo } h \in \mathfrak{h}\}
$$

que é um h-submódulo de $V$.

Definição 2.2.2. Seja $\mathfrak{h}$ uma álgebra de Lie abeliana, e seja $\pi: \mathfrak{h} \longrightarrow \mathfrak{g l}(V)$ uma representação de $\mathfrak{h}$ em um $\mathbb{C}$-espaço vetorial $V$. Dizemos que o $\mathfrak{h}$-módulo $V$ admite uma decomposição em espaços 
de peso se $V=\bigoplus_{\phi \in \mathfrak{h}^{*}} V_{\phi}$. Os funcionais lineares $\phi \in \mathfrak{h}^{*}$ para os quais $V_{\phi} \neq\{0\}$ são chamados de pesos de $V$ relativos a h e $\pi$. Para cada $\phi \in \mathfrak{h}^{*}$, dim $V_{\phi}$ é chamada de multiplicidade de $\phi$ em $V$ e denotada por mult ${ }_{V}(\phi)$. Denotamos por $P(V)$ o conjunto de todos os pesos de $V$.

Proposição 2.2.3. Sejam $\mathfrak{g}$ uma álgebra de Lie, e $\pi: \mathfrak{h} \longrightarrow$ Derg uma representação de uma álgebra de Lie abeliana $\mathfrak{h}$ como uma álgebra de Lie de derivações em $\mathfrak{g}$. Se $\mathfrak{g}$ admite uma decomposição em espaços de peso

$$
\mathfrak{g}=\bigoplus_{\alpha \in \mathfrak{h}^{*}} \mathfrak{g}_{\alpha}
$$

com respeito a h sob a representação $\pi$, então

$$
\left[\mathfrak{g}_{\alpha}, \mathfrak{g}_{\beta}\right] \subset \mathfrak{g}_{\alpha+\beta},
$$

para todo $\alpha, \beta \in \mathfrak{h}^{*}$.

Demonstração. Como $\pi(h) \in \operatorname{Der} \mathfrak{g}$, para todo $h \in \mathfrak{h}$, então $\pi(h)[x, y]=[\pi(h) x, y]+[x, \pi(h) y]$, para todo $x, y \in \mathfrak{g}$. Em particular, para $x \in \mathfrak{g}_{\alpha}$ e $y \in \mathfrak{g}_{\beta}$,

$$
\pi(h)[x, y]=[\alpha(h) x, y]+[x, \beta(h) y]=(\alpha(h)+\beta(h))[x, y]=(\alpha+\beta)(h)[x, y] \in \mathfrak{g}_{\alpha+\beta},
$$

como desejado.

Com as definições e notação estabelecidas até aqui, passaremos à definição e ao estudo da decomposição triangular propriamente dita.

Definição 2.2.4. Seja $\mathfrak{g}$ uma álgebra de Lie. Uma decomposição triangular de $\mathfrak{g}$ consiste de uma subálgebra abeliana $\mathfrak{h} \neq\{0\}$ e duas subálgebras $\mathfrak{g}_{+} e \mathfrak{g}_{-}$tais que

(a) $\mathfrak{g}=\mathfrak{g}_{-} \oplus \mathfrak{h} \oplus \mathfrak{g}_{+}$

(b) $\mathfrak{g}_{+} \neq\{0\},\left[\mathfrak{h}, \mathfrak{g}_{+}\right] \subset \mathfrak{g}_{+}$, e $\mathfrak{g}_{+}$admite uma decomposição em espaços de peso relativa a $\mathfrak{h}$ (sob a representação adjunta) com pesos $\alpha \neq 0$ pertencendo a um semigrupo aditivo livre $Q_{+} \subset \mathfrak{h}^{*}$;

(c) existe uma anti-involução $\sigma$ (isto é, um antiautomorfismo de período 2) em $\mathfrak{g}$ tal que

$$
\begin{aligned}
\sigma\left(\mathfrak{g}_{+}\right) & =\mathfrak{g}_{-}, \\
\sigma \uparrow_{\mathfrak{h}} & =I d_{\mathfrak{h}}
\end{aligned}
$$

(d) existe uma base $\left\{\alpha_{j}\right\}_{j \in J}$ de $Q_{+}$consistindo de elementos linearmente independentes de $\mathfrak{h}^{*}$. Em particular, $Q_{+}$consiste de todas as somas finitas não nulas da forma

$$
\sum_{j \in J} m_{j} \alpha_{j}, m_{j} \in \mathbb{N}
$$


Uma decomposição triangular é dita regular se os espaços de peso de $\mathfrak{g}_{+}$em (b) são de dimensão finita.

Observação 2.2.5. Podemos obter uma definição mais geral de decomposição triangular retirando a condição (d) e não exigindo em (b) que os pesos $\alpha \neq 0$ pertençam a um semigrupo aditivo livre $Q_{+} \subset \mathfrak{h}^{*}$

Como consequência direta da definição, temos:

(1) a condição $0 \notin Q_{+}$é equivalente a $Q_{+} \cap\left(-Q_{+}\right)=\emptyset$ e

(2) $\sigma\left(\mathfrak{g}_{-}\right)=\mathfrak{g}_{+}$, pois $\sigma$ é um antiautomorfismo de período 2 .

Observação 2.2.6. Seja g uma álgebra de Lie admitindo uma decomposição triangular. Consideremos $x \in\left(\mathfrak{g}_{+}\right)_{\alpha}, \alpha \in Q_{+}$. Então $[h, \sigma x]=[\sigma h, \sigma x]=\sigma[x, h]=\sigma(-[h, x])=-\alpha(h) \sigma x$, para todo $h \in \mathfrak{h}$, isto é, $\sigma x$ é um elemento de $\left(\mathfrak{g}_{-}\right)_{-\alpha}$. Portanto, $\mathfrak{g}_{-}$admite uma decomposição em espaços de peso relativa a $\mathfrak{h}$, com pesos em $-Q_{+}$. Ainda,

(a)

$$
\begin{aligned}
\left(\mathfrak{g}_{-}\right)_{-\alpha} & =\sigma\left(\mathfrak{g}_{+}\right)_{\alpha}, \\
\operatorname{dim}\left(\mathfrak{g}_{-}\right)_{-\alpha} & =\operatorname{dim}\left(\mathfrak{g}_{+}\right)_{\alpha}, \text { para todo } \alpha \in Q_{+} ;
\end{aligned}
$$

e $\mathfrak{g}$ admite um decomposição em espaços de peso relativa a $\mathfrak{h}$ com

(b)

$$
\mathfrak{g}_{\alpha}=\left\{\begin{array}{ccc}
\left(\mathfrak{g}_{+}\right)_{\alpha}, & \text { se } & \alpha \in Q_{+}, \\
\left(\mathfrak{g}_{-}\right)_{\alpha}, & \text { se } & -\alpha \in Q_{+}, \\
\mathfrak{h}, & \text { se } & \alpha=0, \\
0 & & \text { caso contrário. }
\end{array}\right.
$$

Observação 2.2.7. Claramente, $\mathfrak{h} \subset \mathfrak{g}_{0}$, mas não é imediato que $\mathfrak{h}=\mathfrak{g}_{0}$. Porém, se mostramos que $\mathfrak{g}=\bigoplus_{\alpha \in Q} \mathfrak{g}_{\alpha}$, em que $Q=\bigoplus_{j \in J} \mathbb{Z} \alpha_{j}$, teremos o resultado desejado, já que $\mathfrak{h} \subset \mathfrak{g}_{0}$.

Proposição 2.2.8. Seja $\mathfrak{g}$ uma álgebra de Lie admitindo uma decomposição triangular. Então, com a notação utilizada na Definição 2.2.4, existe uma decomposição em espaços de peso $\mathfrak{g}=\bigoplus_{\alpha \in Q} \mathfrak{g}_{\alpha}$.

Demonstração. Como $\mathfrak{h} \subset \mathfrak{g}_{0}$ e tanto $\mathfrak{g}_{-}$como $\mathfrak{g}_{+}$possuem decomposição em espaços de peso, já temos que $\mathfrak{g}=\sum_{\alpha \in Q} \mathfrak{g}_{\alpha}$, e precisamos apenas mostrar que a soma é direta.

Para tanto, iremos mostrar que, para cada soma finita da forma $x_{1}+\ldots+x_{k}=0$, com $x_{i} \in \mathfrak{g}_{\beta_{i}}$, com $\beta_{1}, \ldots, \beta_{k}$ distintos, implica $x_{i}=0$, para todo $i$. Vamos então supor que a afirmação não seja verdadeira. Escolhemos um valor mínimo de $k$ para o qual é falsa. Assim, para este $k$, temos que $x_{1}+\ldots+x_{k}=0$, mas não é verdade que $x_{i}=0$ para todo $i$. Então, para todo $h \in \mathfrak{h}$,

$$
0=\left[h, x_{1}+\ldots+x_{k}\right]=\left[h, x_{1}\right]+\ldots+\left[h, x_{k}\right]=\beta_{1}(h) x_{1}+\ldots+\beta_{k}(h) x_{k} .
$$


Temos também que

$$
\beta_{k}(h) x_{1}+\ldots+\beta_{k}(h) x_{k}=0,
$$

de onde

$$
\left(\beta_{1}(h)-\beta_{k}(h)\right) x_{1}+\ldots+\left(\beta_{k-1}(h)-\beta_{k}(h)\right) x_{k-1}=0 .
$$

Pela minimalidade de $k$, temos

$$
\left(\beta_{i}(h)-\beta_{k}(h)\right) x_{i}=0 \text {, para } i=1, \ldots, k-1 .
$$

Como $\beta_{i} \neq \beta_{k}$, existe $h^{\prime} \in \mathfrak{h}$ tal que $\beta_{i}\left(h^{\prime}\right) \neq \beta_{k}\left(h^{\prime}\right)$. Logo, $x_{i}=0$, para $i=1, \ldots, k-1$, o que implica $x_{k}=0$, contrariando o que assumimos no início. Podemos então concluir que a soma é de fato direta.

Definição 2.2.9. Seja $\mathfrak{g}$ uma álgebra de Lie com decomposição triangular. Então, $Q=\bigoplus_{j \in J} \mathbb{Z} \alpha_{j}$ será chamado de reticulado de raizes.

Diremos que os $\alpha \in Q$ tais que $\alpha \neq 0$ e para os quais $\mathfrak{g}_{\alpha} \neq\{0\}$ são raizes de $\mathfrak{g}$ (com relação $a \mathfrak{h})$; e os correspondentes espaços $\mathfrak{g}_{\alpha}$ são os espaços de raízes. Para cada raiz $\alpha$, a dimensão de $\mathfrak{g}_{\alpha}$ será chamada de multiplicidade de $\alpha$. Como usual, chamaremos de sistema de raízes ao conjunto $\Delta$ de todas as raizes de $\mathfrak{g}$; definindo $\Delta \cap Q_{+}=\Delta_{+} e \Delta_{-}=-\Delta_{+}$, temos respectivamente os conjuntos das raízes positivas e negativas de $\mathfrak{g}$.

$\mathrm{Na}$ bibliografia, em geral, podemos encontrar autores que consideram $\alpha=0$ como raiz. Aqui, para manter a uniformidade da notação que está sendo utilizada para as álgebras de Kac-Moody, não consideraremos 0 como uma raiz de $\mathfrak{g}$.

Exemplo 2.2.10. Seja $\mathfrak{g}$ uma álgebra de Lie com decomposição triangular $\mathfrak{g}=\mathfrak{g}_{-} \oplus \mathfrak{h} \oplus \mathfrak{g}_{+}$. Então, nessas condições, $\mathfrak{h}$ é uma subálgebra de Cartan de $\mathfrak{g}$. Pela Definição 2.2.4, temos que h é uma álgebra abeliana e, portanto, nilpotente. Assim, iremos mostrar apenas que h é seu próprio normalizador. De fato, consideremos um elemento $x \in \mathfrak{g}$ tal que $[x, h] \in \mathfrak{h}$, para todo $h \in \mathfrak{h}$. Como, por hipótese, $\mathfrak{g}$ admite uma decomposição triangular, então $x$ se escreve de forma única como $x=x_{1}+h^{\prime}+x_{2}$, com $x_{1} \in \mathfrak{g}_{-}, h^{\prime} \in \mathfrak{h}$ e $x_{2} \in \mathfrak{g}_{+}$. Temos então

$$
\left[x_{1}+h^{\prime}+x_{2}, h\right]=\left[x_{1}, h\right]+\left[x_{2}, h\right] \in \mathfrak{h} \cap\left(\mathfrak{g}_{-}+\mathfrak{g}_{+}\right),
$$

o que nos permite concluir que $[x, h]=0$, para todo $h \in \mathfrak{h}$ e, portanto, $x \in \mathfrak{g}_{0}$. Como $\mathfrak{g}_{0}=\mathfrak{h}$, temos que $\mathfrak{h}$ é seu próprio normalizador.

Exemplo 2.2.11. Consideremos $\mathfrak{g}=\mathfrak{s l}(n, \mathbb{C})$, com $n>1$. Sejam $\mathfrak{g}_{+}$a subálgebra de $\mathfrak{g}$ das matrizes triangulares estritamente superiores, $\mathfrak{g}_{-}$a subálgebra das matrizes triangulares estritamente inferiores e $\mathfrak{h}$ a subálgebra das matrizes diagonais, que claramente é uma subálgebra abeliana. Conhecemos a decomposição

$$
\mathfrak{g}=\mathfrak{g}_{-} \oplus \mathfrak{h} \oplus \mathfrak{g}_{+},
$$


que mostraremos ser de fato uma decomposição triangular.

$$
\begin{aligned}
\sigma: \mathfrak{g} & \longrightarrow \mathfrak{g} \\
A & \longmapsto A^{t}
\end{aligned}
$$

é a anti-involução da definição, pois fixa os pontos de $\mathfrak{h}$, e permuta $\mathfrak{g}_{+}$e $\mathfrak{g}_{-}$.

Para determinar a decomposição em espaços de peso, introduzimos as matrizes unitárias $E_{i j}$, $1 \leq i, j \leq n$ : a matriz com 1 na entrada $(i, j)$ e 0 nas outras. Sejam $\varepsilon_{1}, \ldots, \varepsilon_{n} \in \mathfrak{g l}(n, \mathbb{C})^{*}$ definidos por

$$
\varepsilon_{i}\left(E_{j j}\right)=\delta_{i j}
$$

e sejam $\alpha_{1}, \ldots, \alpha_{n-1}$ definidos por

$$
\alpha_{i}:=\left(\varepsilon_{i}-\varepsilon_{i+1}\right) \uparrow_{\mathfrak{h}}
$$

Como

$$
\left\{\left(\sum a_{i} \varepsilon_{i}\right) \vdash_{\mathfrak{h}} \mid a_{i} \in \mathbb{C}, \sum a_{i}=0\right\}=\mathfrak{h}^{*}
$$

já que $\varepsilon_{1}+\ldots+\varepsilon_{n}$ é o funcional nulo quando restrito a $\mathfrak{h}$, então $\alpha_{1}, \ldots, \alpha_{n-1}$ é uma base de $\mathfrak{h}^{*}$. Para $h \in \mathfrak{h}$,

$$
\left[h, E_{i j}\right]=h E_{i j}-E_{i j} h=\left(\varepsilon_{i}-\varepsilon_{j}\right)(h) E_{i j}
$$

e, em particular, para $i<j,\left[h, E_{i j}\right]=\left(\alpha_{i}+\ldots+\alpha_{j-1}\right)(h) E_{i j}$. Assim, $E_{i j} \in \mathfrak{g}_{+}$é um vetor de peso com peso $\alpha_{i}+\ldots+\alpha_{j-1}$.

Definimos então $Q_{+}:=\left\{\sum m_{i} \alpha_{i} \mid m_{i} \in \mathbb{N}\right\}$ e

$$
\mathfrak{g}_{+}=\bigoplus_{\alpha \in Q_{+}}\left(\mathfrak{g}_{+}\right)_{\alpha}=\bigoplus_{i<j} \mathbb{C} E_{i j}
$$

é a decomposição em espaços de peso. Temos ainda que $E_{j i}=E_{i j}^{t}$ tem peso $-\left(\alpha_{i}+\ldots+\alpha_{j-1}\right)$, para todo $i<j$ e

$$
\mathfrak{g}_{-}=\bigoplus_{i>j} \mathbb{C} E_{i j}
$$

é a decomposição em espaços de peso de $\mathfrak{g}_{-}$. Assim, temos uma decomposição triangular regular para $\mathfrak{g}=\mathfrak{s l}(n, \mathbb{C})$.

Observação 2.2.12. Notemos que, para o caso especial em que $\mathfrak{g}=\mathfrak{s l}(2, \mathbb{C})$, temos

$$
\mathfrak{g}=\mathbb{C} f \oplus \mathbb{C} h \oplus \mathbb{C} e,
$$

em que $\{e, f, h\}$ é a base padrão de $\mathfrak{g}$, ou seja, $\mathfrak{h}=\mathbb{C} h$. Assim, $Q_{+}=\mathbb{N} \alpha$, em que $\alpha: \mathfrak{h} \longrightarrow \mathbb{C}$ e $\alpha(h)=2$. 
A partir de agora, apresentaremos algumas definições e resultados fundamentais para o estudo dos módulos de Verma.

Definição 2.2.13. Seja $\mathfrak{g}$ uma álgebra de Lie admitindo uma decomposição triangular, e escreva $\mathfrak{g}=\mathfrak{g}_{-} \oplus \mathfrak{h} \oplus \mathfrak{g}_{+}$. Seja $\pi$ uma representação de $\mathfrak{g}$ em um $\mathbb{C}$-espaço vetorial $V$. Um vetor não nulo $v \in V$ é chamado de vetor de peso máximo para $\mathfrak{g}$ (relativo à decomposição triangular) se:

(a) v é um vetor de peso relativo à ação de $\mathfrak{h}$.

(b) $\pi(x) v=0$, para todo $x \in \mathfrak{g}_{+}$.

Algumas vezes, principalmente na literatura física, um vetor de peso máximo é também chamado de vetor de vácuo ou vetor nulo.

Definição 2.2.14. Um g-módulo $V$ é dito ser um módulo de peso máximo se $V$ contém um vetor de peso máximo v que o gera, isto é,

$$
\pi(\mathfrak{U}(\mathfrak{g})) v:=\{\pi(u) v \mid u \in \mathfrak{U}(\mathfrak{g})\}=V
$$

observando que aqui estamos usando a notação $\pi$ também para a representação de $\mathfrak{U}(\mathfrak{g})$ em $V$ fornecida pela representação de $\mathfrak{g}$ em $V$. O peso $\lambda \in \mathfrak{h}^{*}$ do vetor de peso máximo $v$ é chamado de peso máximo de $V$. A representação de g em $V$ é chamada de representação de peso máximo.

Notemos que, dada a decomposição triangular $\mathfrak{g}=\mathfrak{g}_{-} \oplus \mathfrak{h} \oplus \mathfrak{g}_{+}$, pelo Teorema A.3.5, temos uma correspondente decomposição triangular da álgebra envolvente universal de $\mathfrak{g}$

$$
\mathfrak{U}(\mathfrak{g})=\mathfrak{U}\left(\mathfrak{g}_{-}\right) \mathfrak{U}(\mathfrak{h}) \mathfrak{U}\left(\mathfrak{g}_{+}\right),
$$

o que nos permite substituir a condição $\pi(\mathfrak{U}(\mathfrak{g})) v=V$ por $\pi\left(\mathfrak{U}\left(\mathfrak{g}_{-}\right)\right) v$.

Observação 2.2.15. Conforme Proposição 2.2 .1 em [18, p.104], se g é uma álgebra de Lie admitindo uma decomposição triangular $\mathfrak{g}=\mathfrak{g}_{-} \oplus \mathfrak{h} \oplus \mathfrak{g}_{+}$, com reticulado de raízes denotado por $Q$, então qualquer módulo $V$ de peso máximo $\lambda$, e vetor de peso máximo $v$, admite uma decomposição em espaços de peso relativa a $\mathfrak{h}$, com todos os pesos no conjunto $\{\lambda\} \cup\left\{\lambda-\beta \mid \beta \in Q_{+}\right\}$. Além disso, $V_{\lambda}=\mathbb{C} v$. Se a decomposição triangular de $\mathfrak{g}$ for regular, ainda teremos todos os espaços de peso com dimensão finita.

\subsection{Módulos de Verma}

Nesta seção serão definidos os módulos de Verma sobre álgebras de Lie com decomposição triangular. Mostraremos ainda, de forma construtiva, a existência desses módulos, isto é, dado $\lambda \in \mathfrak{h}^{*}$, existe um módulo de Verma de peso máximo $\lambda$ associado a ele.

Observação 2.3.1. Notação: Sejam $\mathfrak{g}$ uma álgebra de Lie, e $\pi$ uma representação de $\mathfrak{g}$ em um espaço vetorial $V$. Sabemos que os conceitos de módulo e representação são equivalentes. Assim, quando estivermos interessados em estudar $V$ como módulo sobre $\mathfrak{g}$, usaremos a notação $x v$, ao invés de $\pi(x) v$, para indicar a ação de um elemento da álgebra em um elemento de $V$. 
Definição 2.3.2 (Módulos de Verma). Sejam $\mathfrak{g}$ uma álgebra de Lie admitindo uma decomposição triangular $\mathfrak{g}=\mathfrak{g}_{-} \oplus \mathfrak{h} \oplus \mathfrak{g}_{+}$, e $\lambda \in \mathfrak{h}^{*}$. Um $\mathfrak{g}$-módulo $M(\lambda)$ de peso máximo $\lambda$, com vetor de peso máximo $v_{\lambda}$ é chamado de módulo de Verma se, dado qualquer outro $\mathfrak{g}$-módulo $M$ de peso máximo, com peso máximo $\lambda$ e vetor de peso máximo $v_{\lambda}^{\prime}$, existe um homomorfismo de $\mathfrak{g}$-módulos $\eta: M(\lambda) \longrightarrow M$ tal que $v_{\lambda} \longmapsto v_{\lambda}^{\prime}$.

Um módulo de Verma é universal no sentido de que cada g-módulo de peso máximo com o mesmo peso máximo é uma imagem homomórfica dele.

Proposição 2.3.3. Seja $\mathfrak{g}$ uma álgebra de Lie com decomposição triangular $\mathfrak{g}=\mathfrak{g}_{-} \oplus \mathfrak{h} \oplus \mathfrak{g}_{+}$. Então, para todo $\lambda \in \mathfrak{h}^{*}$, g possui um módulo de Verma $M(\lambda)$ de peso máximo $\lambda$.

Demonstração. Seja $\mathfrak{b}$ a subálgebra $\mathfrak{h} \oplus \mathfrak{g}_{+}$de $\mathfrak{g}$. Seja $\mathbb{C} v_{\lambda}$ um $\mathbb{C}$-espaço vetorial unidimensional, ao qual podemos dar uma estrutura de $\mathfrak{b}$-módulo à esquerda, definindo uma multiplicação por um elemento de $\mathfrak{b}$ :

$$
\begin{aligned}
h v_{\lambda} & =\lambda(h) v_{\lambda}, \text { para todo } h \in \mathfrak{h}, \\
\mathfrak{g}_{+} v_{\lambda} & =0 .
\end{aligned}
$$

Dos resultados para a álgebra envolvente universal, temos os seguintes fatos:

(a) $\mathbb{C} v_{\lambda}$ é um $\mathfrak{U}(\mathfrak{b})$-módulo à esquerda;

(b) $\mathfrak{U}(\mathfrak{g})$ tem estrutura de $\mathfrak{U}(\mathfrak{b})$-módulo à direita.

Pode-se então formar o módulo induzido obtido de $\mathbb{C} v_{\lambda}$

$$
M(\lambda):=\mathfrak{U}(\mathfrak{g}) \otimes_{\mathfrak{U}(\mathfrak{b})} \mathbb{C} v_{\lambda},
$$

que é um $\mathfrak{U}(\mathfrak{g})$-módulo à esquerda (pelo Lema 2.1.6) que, novamente pelos resultados para álgebras envolventes, é um g-módulo à esquerda. Considere $1 \otimes v_{\lambda} \in M(\lambda)$. Para $x \in \mathfrak{g}_{+}$,

$$
x\left(1 \otimes v_{\lambda}\right)=1 \otimes x v_{\lambda}=0,
$$

e para todo $h \in \mathfrak{h}$,

$$
h\left(1 \otimes v_{\lambda}\right)=1 \otimes h v_{\lambda}=1 \otimes \lambda(h) v_{\lambda}
$$

Temos também que $\mathfrak{U}(\mathfrak{g})\left(1 \otimes v_{\lambda}\right)=\mathfrak{U}(\mathfrak{g}) \otimes_{\mathfrak{U}(\mathfrak{b})} \mathbb{C} v_{\lambda}=M(\lambda)$. Assim, $M(\lambda)$ é um módulo de peso máximo, com vetor de peso máximo $1 \otimes v_{\lambda}$, de peso máximo $\lambda$.

Seja agora $M^{\prime}$ um outro módulo de peso máximo, com vetor de peso máximo $v_{\lambda}^{\prime}$ e peso máximo $\lambda$. A função

$$
\begin{aligned}
f: \mathfrak{U}(\mathfrak{g}) \times \mathbb{C} v_{\lambda} & \longrightarrow M^{\prime} \\
\left(u, \alpha v_{\lambda}\right) & \longmapsto \alpha u v_{\lambda}^{\prime},
\end{aligned}
$$


é $\mathfrak{U}(\mathfrak{b})$-biaditiva. De fato,

$$
\begin{aligned}
f\left(u_{1}+u_{2}, \alpha v_{\lambda}\right) & =\left(\alpha\left(u_{1}+u_{2}\right)\right) v_{\lambda}^{\prime}=\left(\alpha u_{1}+\alpha u_{2}\right) v_{\lambda}^{\prime}=\left(\alpha u_{1}\right) v_{\lambda}^{\prime}+\left(\alpha u_{2}\right) v_{\lambda}^{\prime} \\
& =f\left(u_{1}, \alpha v_{\lambda}\right)+f\left(u_{2}, \alpha v_{\lambda}\right) \\
f\left(u, \alpha v_{\lambda}+\beta v_{\lambda}\right) & =((\alpha+\beta) u) v_{\lambda}^{\prime}=(\alpha u) v_{\lambda}^{\prime}+(\beta u) v_{\lambda}^{\prime}=f\left(u, \alpha v_{\lambda}\right)+f\left(u, \beta v_{\lambda}\right) \\
f\left(u b, \alpha v_{\lambda}\right) & =(\alpha(u b)) v_{\lambda}^{\prime}=((\alpha u) b) v_{\lambda}^{\prime}=(\alpha u)\left(b v_{\lambda}^{\prime}\right)=f\left(u, \alpha\left(b v_{\lambda}\right)\right)=f\left(u, b\left(\alpha v_{\lambda}\right)\right),
\end{aligned}
$$

para todo $u, u_{1}, u_{2} \in \mathfrak{U}(\mathfrak{g}), \alpha, \beta \in \mathbb{C}, b \in \mathfrak{U}(\mathfrak{b})$.

Assim, existe um único $\mathbb{Z}$-homomorfismo $g: \mathfrak{U}(\mathfrak{g}) \otimes_{\mathfrak{U}(\mathfrak{b})} \mathbb{C} v_{\lambda} \longrightarrow M^{\prime}$ tal que $g \circ \otimes=f$, isto é, $g\left(1 \otimes v_{\lambda}\right)=f\left(1, v_{\lambda}\right)=v_{\lambda}^{\prime}$, que é um homomorfismo sobrejetor de $\mathfrak{U}(\mathfrak{g})$-módulos.

Usando a universalidade, temos também a unicidade, a menos de isomorfismo, de um módulo de Verma de peso máximo $\lambda$ sobre $\mathfrak{g}$.

Estudaremos agora alguns resultados clássicos a respeito da estrutura dos módulos de Verma.

Proposição 2.3.4. Seja $\mathfrak{g}$ uma álgebra de Lie com decomposição triangular $\mathfrak{g}=\mathfrak{g}_{-} \oplus \mathfrak{h} \oplus \mathfrak{g}_{+}$, $e$ seja $\lambda \in \mathfrak{h}^{*}$.

(i) Se $M(\lambda)$ é o módulo de Verma de peso máximo $\lambda$, então

(ia) cada submódulo próprio $N$ de $M(\lambda)$ possui uma decomposição em espaços de peso relativa $a \mathfrak{h}$, e os pesos estão em $\lambda-Q_{+}$, isto é, os pesos são da forma $\lambda-\alpha$, com $\alpha \in Q_{+}$;

(ib) $M(\lambda)$ possui um único submódulo próprio maximal $N(\lambda)$;

(ic) $L(\lambda):=M(\lambda) / N(\lambda)$ é irredutivel e, a menos de isomorfismo, $L(\lambda)$ é o único módulo de peso máximo irredutível de $\mathfrak{g}$, com peso máximo $\lambda$;

(ii) Qualquer módulo de peso máximo $M$ com peso máximo $\lambda$ contém um único submódulo próprio maximal $N$. Além disso, $M / N \cong L(\lambda)$.

Demonstração. (i) (ia) Seja $N$ um g-submódulo de $M(\lambda)$. Em particular, $N$ é um hy-submódulo de $M(\lambda)$. Já sabemos que $M(\lambda)$ admite uma decomposição em espaços de peso relativa a h. Assim, conforme Proposição 2.1 .1 em ([18, p.92]), $N$ admite uma decomposição em espaços de peso $N_{\phi}=M_{\phi} \cap N$, para todo $\phi \in \mathfrak{h}^{*}$. Quando $\lambda$ é um peso de $N$, temos que $N_{\lambda}=M(\lambda)_{\lambda}=\mathbb{C} v_{\lambda}$, em que $v_{\lambda}$ é o vetor de peso máximo de $M(\lambda)$, e então $N=M$, visto que $v_{\lambda}$ gera $M(\lambda)$ como $\mathfrak{g}$-módulo. Portanto, se $N$ é um submódulo próprio, todos os pesos estão em $\lambda-Q_{+}$.

(ib) Seja $N(\lambda)$ a soma de todos os $\mathfrak{g}$-submódulos próprios de $M(\lambda)$. Conforme vimos na demonstração do item anterior, $\lambda$ não é um peso para nenhum deles; consequentemente, também não o é para $N(\lambda)$, já que nenhum submódulo próprio poderá contribuir para $M(\lambda)_{\lambda}$. Assim, $N(\lambda)$ é um submódulo próprio de $M(\lambda)$ com as características desejadas. 
(ic) $L(\lambda)=M(\lambda) / N(\lambda)$ é um módulo de peso máximo com peso máximo $\lambda$, e é irredutível, pois $N(\lambda)$ é maximal. Precisamos mostrar que $L(\lambda)$ é, a menos de isomorfismo, o único nessas condições.

Seja então $L^{\prime}$ um outro módulo de peso máximo, irredutível, com peso máximo $\lambda$. Como $M(\lambda)$ é um módulo de Verma, existe um homomorfismo sobrejetivo

$$
\eta: M(\lambda) \longrightarrow L^{\prime}
$$

tal que $\eta\left(M(\lambda)_{\phi}\right)=L_{\phi}^{\prime}$, para todos os pesos $\phi$ de $M(\lambda)$. Pelo Teorema Fundamental do Isomorfismo para Módulos, $M(\lambda) / K e r \eta \cong L^{\prime}$. Como $L^{\prime}$ é irredutível, $M(\lambda) /$ Ker $\eta$

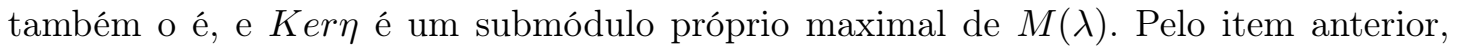
$\eta(N(\lambda))=\{0\}$ e $M(\lambda) / N(\lambda) \cong L^{\prime}$.

(ii) Seja $M$ um módulo de peso máximo $\lambda$. Como $M(\lambda)$ é um módulo de Verma, pela propriedade universal, existe um homomorfismo sobrejetor $M(\lambda) \longrightarrow M$, que leva espaços de peso em espaços de peso. Assim, a imagem de $N(\lambda)$ por esse homomorfismo é um submódulo próprio $N$ de $M$. Então, $N$ é maximal, e $M / N \cong M(\lambda) / N(\lambda)=L(\lambda)$.

Proposição 2.3.5. Sejam $\mathfrak{g}$ uma álgebra de Lie admitindo uma decomposição triangular

$$
\mathfrak{g}=\mathfrak{g}_{-} \oplus \mathfrak{h} \oplus \mathfrak{g}_{+},
$$

e $\mu, \lambda \in \mathfrak{h}^{*}$. Então, qualquer homomorfismo não nulo $\varphi: M(\mu) \longrightarrow M(\lambda)$ é injetivo.

Demonstração. Sejam $v_{\mu}, v_{\lambda}$ os vetores de peso máximo que geram $M(\mu)$ e $M(\lambda)$, respectivamente. Pela definição de módulos de Verma, sabemos que $M(\lambda)=\mathfrak{U}\left(\mathfrak{g}_{-}\right) v_{\lambda}$; assim, $\varphi\left(v_{\mu}\right)=u v_{\lambda}$, para um único $u \in \mathfrak{U}\left(\mathfrak{g}_{-}\right)$. Por outro lado, $\varphi\left(u^{\prime} v_{\mu}\right)=u^{\prime} \varphi\left(v_{\mu}\right)=u^{\prime} u v_{\lambda}$, para todo $u^{\prime} \in \mathfrak{U}\left(\mathfrak{g}_{-}\right)$. Assim, $\varphi$ é unicamente determinada por $u$ e podemos ver que $\varphi$ corresponde a uma aplicação $\mathfrak{U}\left(\mathfrak{g}_{-}\right) \longrightarrow \mathfrak{U}\left(\mathfrak{g}_{-}\right)$. Assim, como a álgebra envolvente universal de uma álgebra de Lie não possui divisores de zero, temos que $\operatorname{Ker} \varphi=\{0\}$ e, portanto, $\varphi$ é injetora.

\subsubsection{A Categoria $\mathcal{O}$}

Ao estudarmos os módulos de Verma, não há como não passarmos pelas representações das álgebras de Kac-Moody, especificamente pelos seus módulos de peso máximo. Para tanto, conforme [14], introduzimos uma categoria de módulos sobre essas álgebras, que dá nome a esta subseção.

Seja $\mathfrak{g}=\mathfrak{g}(A)$ a álgebra de Lie associada à uma matriz complexa $n \times n$, como definido no Capítulo 1, que possui uma decomposição triangular:

$$
\mathfrak{g}=\mathfrak{n}_{-} \oplus \mathfrak{h} \oplus \mathfrak{n}_{+}
$$

em que h é uma subálgebra de Cartan de $\mathfrak{g}$. De acordo com o Teorema A.3.5, temos ainda a seguinte decomposição de $\mathfrak{U}(\mathfrak{g})$ : 


$$
\mathfrak{U}(\mathfrak{g})=\mathfrak{U}\left(\mathfrak{n}_{-}\right) \otimes \mathfrak{U}(\mathfrak{h}) \otimes \mathfrak{U}\left(\mathfrak{n}_{+}\right) .
$$

A Categoria $\mathcal{O}$ é então definida: seus objetos são os g-módulos $V$ que admitem uma decomposição em espaços de peso $V=\bigoplus_{\lambda \in \mathfrak{h}^{*}} V_{\lambda}$, com cada um desses espaços de dimensão finita, e tais que existe uma quantidade finita de elementos $\lambda_{1}, \ldots, \lambda_{s} \in \mathfrak{h}^{*}$ tais que

$$
P(V) \subset \bigcup_{i=1}^{s} D\left(\lambda_{i}\right),
$$

em que $P(V)=\left\{\lambda \in \mathfrak{h}^{*} \mid V_{\lambda} \neq 0\right\}$, e $D(\lambda)=\left\{\mu \in \mathfrak{h}^{*} \mid \mu \leq \lambda\right\}$. Os morfismos são os homomorfismos de $\mathfrak{g}$-módulos.

Os principais exemplos dos objetos desta categoria são os módulos de peso máximo; em particular, os módulos de Verma.

Os módulos de peso máximo para uma álgebra de Kac-Moody, assim como os módulos de Verma, são definidos exatamente da mesma forma que foram definidos para uma álgebra de Lie qualquer.

\subsection{Módulos de Verma Imaginários}

Na seção anterior foram definidos os módulos de Verma sobre álgebras de Lie com decomposição triangular. De certa forma, estava implícita a ideia de que tal decomposição estava diretamente relacionada com o conjunto das raízes positivas. No caso particular das álgebras de Kac-Moody afim esse conjunto é $\Delta_{+} \subset \Delta$.

Em [9] foram estudados os módulos de Verma imaginários sobre álgebras de Kac-Moody afim associados a um outro tipo de subconjunto (fechado) de $\Delta$, que não necessariamente $\Delta_{+}$.

Conforme [14, p.145-146], módulos de Verma sobre álgebras de Kac-Moody possuem decomposição em espaços de peso, em que cada um desses subespaços tem dimensão finita. Veremos que, na decomposição dos módulos de Verma imaginários, aparecem tanto espaços de peso com dimensão finita, como infinita.

Nesta seção, os módulos de Verma imaginários serão definidos, de forma construtiva, e serão enunciados resultados que descrevem os espaços de peso, bem como um critério de irredutibilidade para esses módulos. Em seguida, baseados em [8], estudaremos o caso específico da álgebra de Lie afim $A_{1}^{(1)}$, enunciando também um critério de irredutibilidade.

Iniciando com algumas considerações gerais, sejam $A=\left(a_{i j}\right), 1 \leq i, j \leq n$, uma matriz de Cartan afim, e $\mathfrak{g}=\mathfrak{g}(A)$ a correspondente álgebra de Lie afim com subálgebra de Cartan $\mathfrak{h}$, sistema de raízes $\Delta$ e centro unidimensional $\mathbb{C} c$.

Definição 2.4.1. Seja $P \subset \Delta$. Diremos que $P$ é um subconjunto fechado de $\Delta$ se para todo $\alpha, \beta \in P, \operatorname{com} \alpha+\beta \in \Delta, \alpha+\beta \in P$.

Notemos que, em particular, o conjunto das raízes positivas $\Delta_{+} \subset \Delta$ é um subconjunto fechado, além de possuir a propriedade $\Delta_{+} \cup \Delta_{-}=\Delta, \Delta_{+} \cap \Delta_{-}=\emptyset$. 
Sejam $\left\{\alpha_{0}, \alpha_{1}, \ldots, \alpha_{n}\right\}$ o conjunto das raízes simples de $\Delta$ e $\delta=\sum_{i=0}^{n} k_{i} \alpha_{i}, k_{i} \in \mathbb{N}$, para todo $i \in\{0,1, \ldots, n\}$, a raiz imaginária positiva minimal em $\Delta$. O conjunto das raízes simples pode ser indexado de modo que $k_{0}=1$ e

(a) ou $-\alpha_{0}+\delta \in \Delta$,

(b) ou $\frac{1}{2}\left(-\alpha_{0}+\delta\right) \in \Delta$.

Estudando os diagramas de Dynkin das tabelas (Aff), verifica-se que é sempre possível fazer essa indexação, pois em todos os casos existe pelo menos um $i$ tal que $k_{i}=1$, e para alguma escolha conveniente de $\alpha_{0}$, de modo que $k_{0}=1,-\alpha_{0}+\delta$ ou $\frac{1}{2}\left(-\alpha_{0}+\delta\right)$ será obtido de uma das raízes simples, utilizando a aplicação sucessiva das reflexões fundamentais, quando necessário, o que ilustramos no exemplo abaixo:

Exemplo 2.4.2. (a) Vamos considerar a álgebra de Lie afim associada ao diagrama $D_{4}^{(3)}$ da tabela (Aff 3)

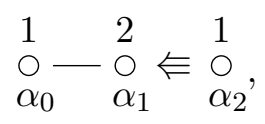

cuja raiz imaginária positiva minimal é $\delta=\alpha_{0}+2 \alpha_{1}+\alpha_{2}$; neste caso, será mantida a indexação original das raízes simples. Ao aplicarmos a reflexão fundamental $r_{2}$ em $\alpha_{1}$ obtemos $\alpha_{1}+\alpha_{2}$; aplicando a reflexão fundamental $r_{1}$ em $\alpha_{1}+\alpha_{2}$ obtemos $2 \alpha_{1}+\alpha_{2}$. Assim, $r_{1}\left(r_{2}\left(\alpha_{1}\right)\right)=-\alpha_{0}+\delta$ é uma raiz real de $\Delta$, já que $r_{1} \circ r_{2}$ é um elemento do grupo de Weyl da álgebra.

(b) Considerando agora a álgebra de Lie afim associada ao diagrama $A_{2}^{(2)}$ da tabela (Aff 2 )

$$
\stackrel{2}{\circ} \stackrel{1}{\circ} \stackrel{\circ}{\circ},
$$

a raiz imaginária positiva minimal é $\delta=2 \alpha_{0}+\alpha_{1}$; neste caso, indexaremos o conjunto das raízes simples trocando $\alpha_{0}$ por $\alpha_{1}$ e iremos obter $-\alpha_{0}+\delta=2 \alpha_{1}$; como $\alpha_{1}$ é uma raiz simples, então $\frac{1}{2}\left(-\alpha_{0}+\delta\right) \in \Delta$.

Seja $\psi=\sum_{i=1}^{n} \alpha_{i}^{*}-\left(\sum_{i=1}^{n} k_{i}\right) \alpha_{0}^{*}$, em que $\alpha_{i}^{*} \in \mathfrak{h}, \alpha_{i}^{*}\left(\alpha_{j}\right)=\delta_{i, j}$. Consideremos o conjunto

$$
N=\{\alpha \in \Delta \mid \psi(\alpha)>0\} \cup\{k \delta \mid k \in \mathbb{N}\} \subset \Delta .
$$

Temos então que $N \cup(-N)=\Delta$ e $N \cap(-N)=\emptyset$, em que

$$
-N=\{\alpha \in \Delta \mid \psi(\alpha)<0\} \cup\{k \delta \mid k \in \mathbb{Z}, k<0\}
$$

Tendo em vista que o conjunto das raízes simples foi indexado de modo que $k_{0}=1$, notemos então que $\psi(\delta)=0$. 
Para cada $F \subset \Delta$, definimos $\mathfrak{g}_{F}=\sum_{\alpha \in F} \mathfrak{g}_{\alpha}$, em que $\mathfrak{g}_{\alpha}=\{x \in \mathfrak{g} \mid[h, x]=\alpha(h) x, \forall h \in \mathfrak{h}\}$. Consideremos então $B_{N}=\mathfrak{g}_{N} \oplus \mathfrak{h}$, que é chamada de subálgebra de Borel imaginária.

Já temos então condições de definir os novos módulos de Verma que são objeto desta seção.

Com as considerações iniciais, temos a seguinte decomposição de $\mathfrak{g}$ :

$$
\mathfrak{g}=\mathfrak{g}_{-N} \oplus B_{N}
$$

Definição 2.4.3. Sejam $\lambda \in \mathfrak{h}^{*}$ e $V$ um $\mathfrak{g}$-módulo. Um vetor não nulo $v \in V$ é chamado de vetor de peso máximo imaginário de peso $\lambda$ se $\mathfrak{g}_{N} v=0$ e $h v=\lambda(h) v$ para todo $h \in \mathfrak{h}$.

Consideremos agora a álgebra envolvente universal $\mathfrak{U}(\mathfrak{g})$ de $\mathfrak{g}, \lambda \in \mathfrak{h}^{*}$, e $\mathbb{C} 1$ (espaço vetorial unidimensional) como um $B_{N}$-módulo sob a ação

$$
(h+x) 1=\lambda(h) 1, \text { para todo } h \in \mathfrak{h}, \text { e } x \in \mathfrak{g}_{N} .
$$

Definimos então o módulo de Verma imaginário

$$
\bar{M}(\lambda)=\mathfrak{U}(\mathfrak{g}) \otimes_{\mathfrak{U}\left(B_{N}\right)} \mathbb{C} 1
$$

associado a $N$ e $\lambda$.

Proposição 2.4.4. ([9])

(a) $\bar{M}(\lambda)$ é um $\mathfrak{U}\left(\mathfrak{g}_{-N}\right)$-módulo livre gerado pelo vetor de peso máximo imaginário $1 \otimes 1$, de peso $\lambda$.

(b) $\operatorname{dim} \bar{M}(\lambda)_{\lambda}=1 ; 0<\operatorname{dim} \bar{M}(\lambda)_{\lambda-k \delta}<\infty$, para todo inteiro $k>0$; se $\bar{M}(\lambda)_{\mu} \neq 0, \mu \neq \lambda-k \delta$ para todos os inteiros $k \geq 0$, então $\operatorname{dim} \bar{M}(\lambda)_{\mu}=\infty$.

(c) Seja $V$ algum $\mathfrak{g}$-módulo gerado por um vetor de peso máximo imaginário $v$ com peso $\lambda$. Então, existe um único homomorfismo sobrejetivo $f: \bar{M}(\lambda) \longrightarrow V$ tal que $f(1 \otimes 1)=v$.

(d) O módulo $\bar{M}(\lambda)$ possui um único submódulo maximal.

(e) Sejam $\lambda, \mu \in \mathfrak{h}^{*}$. Qualquer elemento não nulo de $\operatorname{Hom}_{\mathfrak{g}}(\bar{M}(\lambda), \bar{M}(\mu))$ é injetivo.

O único quociente irredutível de $\bar{M}(\lambda)$ é denotado por $\bar{L}(\lambda)$.

Um resultado primordial em [9] é o critério de irredutibilidade dos módulos de Verma imaginários, fornecido pelo Teorema 1 , a saber: $\bar{M}(\lambda)$ é irredutível se e somente se $\lambda(c) \neq 0$. Além disso, se $\lambda(c)=0$, mas $\lambda\left(h_{i}\right) \neq 0$, para $1 \leq i \leq n$, então o submódulo maximal de $\bar{M}(\lambda)$ é gerado por $\sum_{k=1}^{\infty} \bar{M}(\lambda)_{\lambda-k \delta}$ 


\subsubsection{Módulos de Verma Imaginários sobre a Álgebra de Lie Afim $A_{1}^{(1)}$}

Nesta seção estudaremos os módulos de Verma imaginários para a álgebra de Lie afim $A_{1}^{(1)}$, utilizando a realização que encontra-se em [14]. Seguindo exclusivamente [8], definiremos tais módulos e enunciaremos os teoremas que versam sobre sua irredutibilidade.

Seja $\dot{\mathfrak{g}}=\mathfrak{s l}(2, \mathbb{C})$, com base padrão $\{e, f, h\}$, satisfazendo as relações:

$$
[e, f]=h,[h, e]=2 e \text { e }[h, f]=-2 f .
$$

Consideremos a álgebra dos polinômios de Laurent $\mathbb{C}\left[t, t^{-1}\right]$ e a álgebra de loop

$$
\mathcal{L}(\dot{\mathfrak{g}})=\dot{\mathfrak{g}} \otimes_{\mathbb{C}} \mathbb{C}\left[t, t^{-1}\right]
$$

com colchete de Lie

$$
\left[x \otimes t^{m}, y \otimes t^{n}\right]=[x, y] \otimes t^{m+n},
$$

para todo $x, y \in \dot{\mathfrak{g}}$ e $m, n \in \mathbb{Z}$. Notemos que $\dot{\mathfrak{g}}$ pode ser vista como uma subálgebra de $\mathcal{L}(\dot{\mathfrak{g}})$ através da identificação $x \longmapsto x \otimes 1$, em que $x \in\{e, f, h\}$.

Seja agora a extensão central de $\mathcal{L}(\dot{\mathfrak{g}})$, pelo centro unidimensional $\mathbb{C} c$, isto é, $\tilde{\mathcal{L}}(\dot{\mathfrak{g}})=\mathcal{L}(\dot{\mathfrak{g}}) \oplus \mathbb{C} c$, em que

$$
\left[x \otimes t^{n}, c\right]=0 \text { e }\left[x \otimes t^{n}, y \otimes t^{m}\right]=[x, y] \otimes t^{n+m}+n \delta_{m+n, 0}(x \mid y) c
$$

para todo $x, y \in \dot{\mathfrak{g}}$ e $m, n \in \mathbb{Z}$, em que (. | .) é uma forma simétrica bilinear não degenerada em $\dot{\mathfrak{g}}$. Finalmente, temos a álgebra de Lie afim desejada, que é a extensão de $\tilde{\mathcal{L}}(\dot{\mathfrak{g}})$ por uma derivação $d$ :

$$
\hat{\mathcal{L}}(\dot{\mathfrak{g}})=\tilde{\mathcal{L}}(\dot{\mathfrak{g}}) \oplus \mathbb{C} d
$$

com $[c, d]=0$ e $\left[d, x \otimes t^{n}\right]=n x \otimes t^{n}$, para todo $x \in \dot{\mathfrak{g}}, n \in \mathbb{Z}$. Essa álgebra é denotada por $\hat{\mathfrak{s l}}(2, \mathbb{C})$.

Sejam $\Delta$ o sistema de raízes de $\hat{\mathfrak{s l}}(2, \mathbb{C}), \pi=\{\alpha, \beta\}$ uma base de $\Delta, \delta=\alpha+\beta$ a raiz positiva imaginária minimal e $\Delta^{i m}=\{k \delta \mid k \in \mathbb{Z} \backslash\{0\}\}$ o conjunto das raízes imaginárias em $\Delta$.

Definimos também

$$
P=P_{\pi, \beta}=\{-\beta+k \delta \mid k \in \mathbb{Z}\} \cup\{k \delta \mid k \in \mathbb{N}\},
$$

e temos $\Delta=P \cup-P$ e $P \cap-P=\emptyset$. Para verificar que isso de fato ocorre, basta observar que $\Delta=\Delta^{r e} \cup \Delta^{i m}$, em que $\Delta^{r e}=\{\gamma+n \delta \mid \gamma \in \dot{\mathfrak{g}}, n \in \mathbb{Z}\}$. No caso da álgebra $\hat{\mathfrak{s} l}(2, \mathbb{C})$, seguindo a notação utilizada no capítulo 1 , denotaremos $\alpha=\alpha_{1} \in \dot{\Delta}$ e $\alpha_{0}=\beta$. Logo, $\dot{\Delta}=\{\alpha,-\alpha\}$. 
Temos então a seguinte decomposição de $\hat{\mathfrak{s} l}(2, \mathbb{C})$ em espaços de raízes:

$$
\hat{\mathfrak{s} l}(2, \mathbb{C})=\mathfrak{h} \oplus \sum_{\varphi \in \Delta} \mathfrak{g}_{\varphi}
$$

em que $\mathfrak{h}=\mathbb{C} h \oplus \mathbb{C} c \oplus \mathbb{C} d$ é uma subálgebra de Cartan de $\hat{\mathfrak{s l}}(2, \mathbb{C})$, e

$$
\mathfrak{g}_{\varphi}=\{x \in \hat{\mathfrak{s}} \mathfrak{l}(2, \mathbb{C}) \mid[\hat{h}, x]=\varphi(\hat{h}) x, \text { para todo } \hat{h} \in \mathfrak{h}\}
$$

Cada um dos espaços de raízes $\mathfrak{g}_{\varphi}, \varphi \in \Delta$ tem dimensão 1 , com as seguintes bases $X_{\varphi}$ :

$$
\begin{aligned}
X_{\beta+k \delta} & =f \otimes t^{k+1}, X_{-\beta+k \delta}=e \otimes t^{k-1}, k \in \mathbb{Z} \\
X_{n \delta} & =h \otimes t^{n}, n \in \mathbb{Z} \backslash\{0\} .
\end{aligned}
$$

Para mostrar que os espaços de peso possuem essas bases, utilizaremos a definição do funcional linear $\delta$, conforme Capítulo 1: $\delta(d)=1$ e $\delta\lceil\mathbb{C} h+\mathbb{C} c=0$; utilizaremos também que $\alpha(d)=\alpha(c)=0$. Queremos avaliar como um elemento de $\mathfrak{h}$ age em cada uma dessas bases, e para isso basta avaliar como $h$ e $d$ agem, já que $c$ é um elemento central em $\hat{\mathfrak{s l}}(2, \mathbb{C})$. De fato,

$$
\begin{aligned}
{\left[h, X_{\beta+k \delta}\right] } & =\left[h, f \otimes t^{k+1}\right]=[h, f] \otimes t^{k+1}=-2 f \otimes t^{k+1}=-\alpha(h) f \otimes t^{k+1}=(\beta+k \delta)(h) f \otimes t^{k+1} \\
{\left[d, X_{\beta+k \delta}\right] } & =\left[d, f \otimes t^{k+1}\right]=(k+1) f \otimes t^{k+1}=(k+1) \delta(d) f \otimes t^{k+1}=(\beta+k \delta)(d) f \otimes t^{k+1} \\
{\left[h, X_{-\beta+k \delta}\right] } & =\left[h, e \otimes t^{k-1}\right]=[h, e] \otimes t^{k-1}=2 e \otimes t^{k-1}=\alpha(h) e \otimes t^{k-1}=(-\beta+k \delta)(h) e \otimes t^{k-1} \\
{\left[d, X_{-\beta+k \delta}\right] } & =\left[d, e \otimes t^{k-1}\right]=(k-1) e \otimes t^{k-1}=(k-1) \delta(d) e \otimes t^{k-1}=(-\beta+k \delta)(d) e \otimes t^{k-1} \\
{\left[h, X_{n \delta}\right] } & =\left[h, h \otimes t^{n}\right]=\underbrace{[h, h] \otimes t^{n}=0=n \delta(h) h \otimes t^{n}}_{0} \\
{\left[d, X_{n \delta}\right] } & =\left[d, h \otimes t^{n}\right]=n h \otimes t^{n}=n \delta(d) h \otimes t^{n},
\end{aligned}
$$

e temos o resultado desejado.

Para quaisquer $k, m \in \mathbb{Z}$, os colchetes entre esses elementos das bases são dados por: 


$$
\begin{aligned}
{\left[X_{k \delta}, X_{m \delta}\right] } & =\left[h \otimes t^{k}, h \otimes t^{m}\right]=\underbrace{[h, h]}_{0} \otimes t^{k+m}+k \delta_{k+m, 0} \underbrace{(h \mid h)}_{2} c \\
& =\left\{\begin{array}{cc}
0, & m+k \neq 0 \\
2 k c, & m+k=0
\end{array},\right. \\
{\left[X_{k \delta}, X_{\beta+m \delta}\right] } & =\left[h \otimes t^{k}, f \otimes t^{m+1}\right]=[h, f] \otimes t^{k+m+1}+k \delta_{k+m+1,0} \underbrace{(h \mid f)}_{0} c \\
& =-2 f \otimes t^{k+m+1}=-2 X_{\beta+(k+m) \delta}, \\
{\left[X_{k \delta}, X_{-\beta+m \delta}\right] } & =\left[h \otimes t^{k}, e \otimes t^{m-1}\right]=[h, e] \otimes t^{k+m-1}+k \delta_{k+m-1,0} \underbrace{(h \mid e)}_{0} c \\
& =2 e \otimes t^{k+m-1}=2 X_{-\beta+(k+m) \delta}, \\
{\left[X_{\beta+k \delta}, X_{-\beta+m \delta}\right] } & =\left[f \otimes t^{k+1}, e \otimes t^{m-1}\right]=[f, e] \otimes t^{k+m}+(k+1) \delta_{m+k, 0} \underbrace{(f \mid e)}_{1} c \\
& =\left\{\begin{array}{cc}
-X_{(m+k) \delta}, & m+k \neq 0, \\
-h+(k+1) c, & m+k=0 .
\end{array}\right.
\end{aligned}
$$

Definiremos agora os módulos que são o objeto principal desta subseção. Começaremos considerando as subálgebras (são subálgebras devido às relações $(2.2))$ de $\hat{\mathfrak{s} l}(2, \mathbb{C})$ :

$$
\mathfrak{g}_{+}=\sum_{\varphi \in P} \mathfrak{g}_{\varphi}, \mathfrak{g}_{-}=\sum_{\varphi \in-P} \mathfrak{g}_{\varphi}
$$

Então, temos uma decomposição de $\hat{\mathfrak{s l}}(2, \mathbb{C})$ em uma soma direta de espaços vetoriais:

$$
\hat{\mathfrak{s l}}(2, \mathbb{C})=\mathfrak{g}_{+} \oplus \mathfrak{h} \oplus \mathfrak{g}_{-}
$$

Definição 2.4.5. Seja $V$ um $\hat{\mathfrak{s} l}(2, \mathbb{C})$-módulo. Diremos que $V$ é um módulo de peso se

$$
V=\bigoplus_{\lambda \in \mathfrak{h}^{*}} V_{\lambda}
$$

em que $V_{\lambda}=\{v \in V \mid h v=\lambda(h) v, \forall h \in \mathfrak{h}\}$.

Seja $V$ um módulo de peso sobre $\hat{\mathfrak{s} l}(2, \mathbb{C})$. Denotaremos por $P(V)$ o conjunto de todos os $\mu \in \mathfrak{h}^{*}$ tais que $V_{\mu} \neq\{0\}$.

Definição 2.4.6. Seja $\lambda \in P(V)$. Um elemento não nulo $v \in V_{\lambda}$ é chamado de vetor de vácuo imaginário de peso $\lambda$ se $\mathfrak{g}_{+} v=0$.

Com as definições estabelecidas, podemos construir o módulo de Verma imaginário sobre $\hat{\mathfrak{s} l}(2, \mathbb{C})$, de forma análoga ao que fizemos para os módulos de Verma padrão, como segue:

Sejam $\mathfrak{U}(\hat{\mathfrak{s} l}(2, \mathbb{C}))$ a álgebra envolvente universal de $\hat{\mathfrak{s}}(2, \mathbb{C})$, e $\lambda \in \mathfrak{h}^{*}$. Definimos sobre o espaço vetorial unidimensional $\mathbb{C} 1$ uma estrutura de módulo sobre a subálgebra $\mathfrak{h} \oplus \mathfrak{g}_{+} \operatorname{de} \hat{\mathfrak{s}}(2, \mathbb{C})$, com a 
ação dada por

$$
(h+x) 1=\lambda(h) 1, \text { para todo } x \in \mathfrak{g}_{+}, \text {e } h \in \mathfrak{h} .
$$

Consideremos então o módulo

$$
\tilde{M}(\lambda)=\mathfrak{U}(\hat{\mathfrak{s} l}(2, \mathbb{C})) \otimes_{\mathfrak{U}\left(\mathfrak{h} \otimes \mathfrak{g}_{+}\right)} \mathbb{C} 1
$$

associado com $\pi, \beta, \lambda . \tilde{M}(\lambda)$ é um módulo de peso e $\tilde{M}(\lambda)_{\lambda}=1 \otimes \mathbb{C} \cong \mathbb{C}$.

Proposição 2.4.7. ([8])

(a) $\tilde{M}(\lambda)$ é um $\mathfrak{U}\left(\mathfrak{g}_{-}\right)$-módulo livre gerado pelo vetor de vácuo imaginário $1 \otimes 1$;

(b) $\tilde{M}(\lambda)$ tem um único $\hat{\mathfrak{s}}(2, \mathbb{C})$-submódulo próprio maximal;

(c) $P(\tilde{M}(\lambda))=\{\lambda+k \beta+m \delta \mid k, m \in \mathbb{Z}, k>0\} \cup\{\lambda-n \delta \mid n \geq 0\}$;

(d) $\operatorname{dim} \tilde{M}(\lambda)_{\lambda+k \beta+m \delta}=\infty, k>0$

$$
\operatorname{dim} \tilde{M}(\lambda)_{\lambda-n \beta}=\left\{\begin{array}{cc}
1, & n=0, \quad \tilde{M}(\lambda)_{\lambda}=\mathbb{C}(1 \otimes 1)=1 \otimes \mathbb{C} 1 \cong \mathbb{C} \\
P(n), & n>0,
\end{array}\right.
$$

em que $P(n)$ é a quantidade de partições de $n$.

O módulo $\tilde{M}(\lambda)$ será chamado de módulo de Verma imaginário(IVM). Denotaremos por $\tilde{N}(\lambda)$ o único submódulo próprio maximal, e por $\tilde{L}(\lambda)$ o quociente irredutível $\tilde{M}(\lambda) / \tilde{N}(\lambda)$. Notemos que o quociente irredutível é também um módulo de peso com vetor de vácuo imaginário de peso $\lambda$. Mais especificamente, esse vetor será a imagem do vetor de vácuo imaginário que gera $\tilde{M}(\lambda)$ pelo homomorfismo canônico.

Seguindo ainda as mesmas ideias utilizadas no caso do módulo de Verma padrão, temos o seguinte:

Se existir algum $\hat{\mathfrak{s l}}(2, \mathbb{C})$-módulo de peso $V$ gerado por um vetor de vácuo imaginário $v$ de peso $\lambda$, então existe um único epimorfismo $f: \tilde{M}(\lambda) \longrightarrow V$ tal que $f(1 \otimes 1)=v$

Proposição 2.4.8. Seja $V$ um $\hat{\mathfrak{s} l}(2, \mathbb{C})$-módulo de peso irredutível com vetor de vácuo imaginário de peso $\lambda$. Então,

$$
V \cong \tilde{L}(\lambda)
$$

O teorema a seguir, cuja demonstração encontra-se em ([8]), fornece um critério para a irredutibilidade de $\tilde{M}(\lambda)$.

Teorema 2.4.9. O módulo $\tilde{M}(\lambda)$ é irredutivel se e somente se $\lambda(c) \neq 0$.

A partir daqui, veremos o que ocorre se $\lambda(c)=0$, para $\lambda \in \mathfrak{h}^{*}$; um funcional linear com essa propriedade será denotado por $\lambda_{0}$. Mesmo que já saibamos que nesse caso $\tilde{M}(\lambda)$ não é irredutível, analisaremos a irredutibilidade de quocientes de $\tilde{M}(\lambda)$. 
Consideremos em $\hat{\mathfrak{s}}(2, \mathbb{C})$ o subespaço vetorial

$$
\mathfrak{g}^{+}=\sum_{\varphi \in P \cup \Delta^{i m}} \mathfrak{g}_{\varphi}
$$

e a subálgebra comutativa

$$
\mathfrak{g}^{-}=\sum_{\varphi \in-P \backslash \Delta^{i m}} \mathfrak{g}_{\varphi}
$$

Então, temos a seguinte decomposição de $\hat{\mathfrak{s} l}(2, \mathbb{C})$ como soma direta de espaços vetoriais:

$$
\hat{\mathfrak{s}}(2, \mathbb{C})=\mathfrak{g}^{+} \oplus \mathfrak{h} \oplus \mathfrak{g}^{-}
$$

Definição 2.4.10. Seja $V$ um $\hat{\mathfrak{s l}}(2, \mathbb{C})$-módulo de peso, $\lambda \in P(V)$. Um elemento não nulo $v \in V_{\lambda}$ é chamado de vetor de vácuo imaginário especial (s.i.v.v.) de peso $\lambda$ se $\mathfrak{g}^{+} v=0$.

Seja $\lambda_{0} \in \mathfrak{h}^{*}$ um funcional linear tal que $\lambda_{0}(c)=0$. Definimos então no espaço unidimensional $\mathbb{C} 1$ uma estrutura de $\mathfrak{h} \oplus \mathfrak{g}^{+}$-módulo através da ação

$$
(h+x) 1=\lambda_{0}(h) 1, \text { para todo } h \in \mathfrak{h} \text { e } x \in \mathfrak{g}^{+} .
$$

Em seguida, consideramos o $\hat{\mathfrak{s} l}(2, \mathbb{C})$-módulo

$$
\hat{M}\left(\lambda_{0}\right)=\mathfrak{U}(\hat{\mathfrak{s} l}(2, \mathbb{C})) \bigotimes_{\mathfrak{U}\left(\mathfrak{h} \otimes \mathfrak{g}^{+}\right)} \mathbb{C} 1
$$

Temos então as seguintes propriedades para esse novo módulo que acabamos de construir:

Proposição 2.4.11. (a) $\hat{M}\left(\lambda_{0}\right)$ é um $\hat{\mathfrak{s l}}(2, \mathbb{C})$-módulo de peso gerado pelo s.i.v.v. $1 \otimes 1$;

(b) $\hat{M}\left(\lambda_{0}\right)$ tem um único submódulo maximal $\hat{N}\left(\lambda_{0}\right)$;

(c) Existe um epimorfismo $f: \tilde{M}\left(\lambda_{0}\right) \longrightarrow \hat{M}\left(\lambda_{0}\right)$;

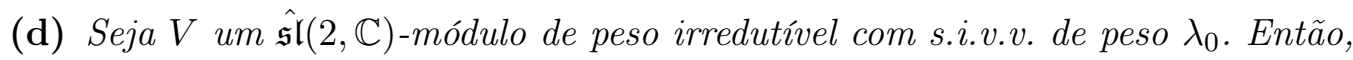

$$
V \cong \hat{M}\left(\lambda_{0}\right) / \hat{N}\left(\lambda_{0}\right) \cong \tilde{L}\left(\lambda_{0}\right)
$$

(e) $P\left(\hat{M}\left(\lambda_{0}\right)\right)=\left\{\lambda_{0}+n \beta+k \delta \mid k \in \mathbb{Z}, n>0\right\} \cup\left\{\lambda_{0}\right\}, \operatorname{dim} \hat{M}\left(\lambda_{0}\right)_{\lambda_{0}+n \beta+k \delta}=\infty, n>1$, $\operatorname{dim} \hat{M}\left(\lambda_{0}\right)_{\lambda_{0}+\beta+k \delta}=\operatorname{dim} \hat{M}\left(\lambda_{0}\right)_{\lambda_{0}}=1$.

E o resultado que buscamos, que é o critério de irredutibilidade para $\hat{M}(\lambda)$ é dado pelo seguinte teorema, conforme [8]: 
Teorema 2.4.12. (a) $\hat{M}\left(\lambda_{0}\right)$ é irredutivel se e somente se $\lambda_{0}(h) \neq 0$;

(b) Se $\lambda_{0}(h)=0$, então $\tilde{L}\left(\lambda_{0}\right)$ é o $\hat{\mathfrak{s} l}(2, \mathbb{C})$-módulo trivial unidimensional.

\subsection{Módulos de Verma J-Imaginários}

Assim como no caso dos módulos de Verma imaginários, o que diferencia a construção dos módulos de Verma J-imaginários da construção dos módulos de Verma, é o tipo de partição (fechada) das raízes que está sendo utilizada.

Nesta seção, com base principalmente em [16], estudaremos os módulos de Verma J-imaginários para a álgebra de Lie afim $\hat{\mathfrak{s l}}(n, \mathbb{C})$, enunciaremos os principais resultados, e exemplificaremos com o caso da álgebra de Lie afim $\hat{\mathfrak{s} l}(2, \mathbb{C})$.

Em primeiro lugar, apresentaremos algumas definições e considerações gerais que serão necessárias para as construções que serão feitas.

Seja $\mathfrak{s l}(n, \mathbb{C})$ a álgebra de Lie complexa das matrizes $n \times n$ com traço zero, com forma de Killing $(X \mid Y)=\operatorname{tr}(X Y)$, para todo $X, Y \in \mathfrak{s l}(n, \mathbb{C})$. Denotando por $E_{i j}$ as matrizes unitárias (que possuem 1 na entrada $(i, j)$ e 0 nas outras), $1 \leq i, j \leq n$, definimos:

$$
H_{i}=E_{i i}-E_{i+1, i+1}, E_{i}=E_{i, i+1}, F_{i}=E_{i+1, i}, i=1, \ldots, n-1 .
$$

Assim, $E_{i}, F_{i}, H_{i}, i=1, \ldots, n-1$, formam um conjunto gerador para $\mathfrak{s l}(n, \mathbb{C})$. Notemos que alguns elementos que fazem parte da base como espaço vetorial não aparecem aqui, pois quando olhamos para a estrutura de álgebra de Lie, esses elementos podem ser obtidos através do colchete entre outros elementos.

Temos então a álgebra de Lie afim $\hat{\mathfrak{s l}}(n, \mathbb{C})=\mathfrak{s l}(n, \mathbb{C}) \otimes \mathbb{C}\left[t, t^{-1}\right] \oplus \mathbb{C} c \oplus \mathbb{C} d$, em que $\mathbb{C} c$ é o centro unidimensional e $d$ uma derivação. Para todo $X \in \mathfrak{s l}(n, \mathbb{C})$ e $m \in \mathbb{Z}$, seja $X_{m}=t^{m} \otimes X$. Então $\hat{\mathfrak{s} l}(n, \mathbb{C})$ é gerada por $E_{i m}, F_{i m}, H_{i m}$, com $1 \leq i \leq n-1, c$ e $d$. Os elementos $H_{i}, i=1, \ldots, n-1$, junto com $c$ e $d$ geram uma subálgebra de Cartan de $\hat{\mathfrak{s l}}(n, \mathbb{C})$.

Fixando $\gamma \in \mathbb{C}^{*}$, e para todo $1 \leq i \leq n-1$, fixemos $\lambda_{i} \in \mathbb{C}$, defina:

$$
\begin{aligned}
& \mathbb{C}[\mathbf{x}]=\mathbb{C}\left[x_{i j, m} \mid i, j, m \in \mathbb{Z}, 1 \leq i, j \leq n-1\right] \\
& \mathbb{C}[\mathbf{y}]=\mathbb{C}\left[y_{i m} \mid i, m \in \mathbb{N}^{*}, 1 \leq i \leq n-1\right],
\end{aligned}
$$

como sendo as álgebras geradas sobre $\mathbb{C}$ por $x_{i j, m}$ e $y_{i m}$, respectivamente. Em $\mathbb{C}[\mathbf{x}]$, defina os operadores

$$
\begin{aligned}
& a_{i j, m}=-x_{i j, m}, \\
& a_{i j, m}^{*}=\frac{\partial}{\partial_{x_{i j,-m}}},
\end{aligned}
$$

em que $\left[a_{i j, m}, a_{k l, p}^{*}\right]=\delta_{i, k} \delta_{j, l} \delta_{m,-p}$. 
Fixe agora $\mathbb{J} \subset \mathbb{N}^{*}$ arbitrário. Em $\mathbb{C}[\mathbf{y}]$, defina operadores $b_{i m}$, com $m \in \mathbb{Z}$ e $1 \leq i \leq n-1$, da seguinte forma:

$$
b_{i m}=\left\{\begin{array}{ccc}
-\gamma^{-1} \lambda_{i} & \text { se } & m=0, \\
-\gamma m \frac{\partial}{\partial_{y_{i m}}} & \text { se } & m \in \mathbb{N}^{*} \backslash \mathbb{J} \\
-\gamma^{-1} y_{i m} & \text { se } & m \in \mathbb{J}, \\
\gamma m \frac{\partial}{\partial_{y_{i,-m}}} & \text { se } & -m \in \mathbb{J}, \\
-\gamma^{-1} y_{i,-m} & \text { se } & -m \in \mathbb{N}^{*} \backslash \mathbb{J},
\end{array}\right.
$$

e temos as relações $\left[b_{i m}, b_{j p}\right]=m \delta_{i, j} \delta_{m,-p}$.

Com isso, temos os elementos necessários para tratar do objeto principal desta seção.

Definição 2.5.1. Seja $\mathbb{J} \subset \mathbb{N}^{*}$ fixado, e seja $\psi_{\mathbb{J}}: \mathbb{Z}^{*} \longrightarrow\{0,1\}$ a função dada por

$$
\psi_{\mathbb{J}}=\left\{\begin{array}{ccc}
1 & \text { se } m \in \mathbb{J} & \text { ou }-m \in \mathbb{N}^{*} \backslash \mathbb{J}, \\
0 & \text { se } m \in \mathbb{N}^{*} \backslash \mathbb{J} & \text { ou }-m \in \mathbb{J} .
\end{array}\right.
$$

Seja agora $\mathfrak{g}=\hat{\mathfrak{s l}}(n, \mathbb{C})$ e consideremos

$$
S_{\psi_{\mathbb{J}}}=\left\{\alpha+m \delta \mid \alpha \in \dot{\Delta}_{+}, m \in \mathbb{Z}\right\} \cup\left\{m \delta \mid m \in \mathbb{N}, \varphi_{\mathbb{J}}(m)=1\right\} \cup\{-m \delta \mid m \in \mathbb{N}, \varphi(m)=0\}
$$

Os espaços

$$
\mathfrak{g}_{S_{\psi_{J}}}=\bigoplus_{\alpha \in S_{\psi_{J}}} \mathfrak{g}_{\alpha} \text { e } \mathfrak{g}_{-S_{\psi_{\mathbb{J}}}}=\bigoplus_{\alpha \in-S_{\psi_{\mathbb{J}}}} \mathfrak{g}_{\alpha}
$$

em que

$$
-S_{\psi_{\mathbb{J}}}=\left\{\alpha+m \delta \mid \alpha \in \dot{\Delta}_{-}, m \in \mathbb{Z}\right\} \cup\left\{m \delta \mid m \in \mathbb{N}, \varphi_{\mathbb{J}}(m)=0\right\} \cup\left\{-m \delta \mid m \in \mathbb{N}, \varphi_{\mathbb{J}}(m)=1\right\},
$$

são subálgebras de fornecem uma decomposição triangular

$$
\mathfrak{g}=\mathfrak{g}_{S_{\psi_{\mathbb{J}}}} \oplus \mathfrak{h} \oplus \mathfrak{g}_{-S_{\psi_{\mathbb{J}}}}
$$

Sejam agora $\lambda \in \mathfrak{h}^{*}$, e $\mathfrak{b}_{\psi_{\mathbb{J}}}=\mathfrak{h} \oplus \mathfrak{g}_{S_{\psi_{J}}}$ a subálgebra de Borel correspondente a $S_{\psi_{\mathbb{J}}}$. Consideremos também um espaço vetorial unidimensional $\mathbb{C} v_{\lambda}$ ao qual é dada uma estrutura de módulo sobre $\mathfrak{b}_{\psi_{J}}$ sob a ação

$$
\begin{aligned}
h v_{\lambda} & =\lambda(h) v_{\lambda}, \text { para todo } h \in \mathfrak{h} \\
\mathfrak{g}_{S_{\psi_{J}}} v_{\lambda} & =0 .
\end{aligned}
$$


Definição 2.5.2. Definimos então o módulo

$$
M_{\psi_{\mathbb{J}}}(\lambda)=M_{\mathfrak{b}_{\psi_{\mathbb{J}}}}(\lambda)=\mathfrak{U}(\mathfrak{g}) \otimes_{\mathfrak{U}\left(\mathfrak{b}_{\psi_{J}}\right)} \mathbb{C} v_{\lambda}
$$

que será chamado de módulo de Verma J-imaginário.

Enunciaremos agora algumas propriedades de $M_{\psi_{\mathbb{J}}}(\lambda)$ :

Proposição 2.5.3 (([2]),Proposição 3.4$)$. Seja $\lambda \in \mathfrak{h}^{*}$ tal que $\lambda(c)=a$. Se $a \neq 0$, então $M_{\psi_{\mathbb{J}}}(\lambda)$ tem as seguintes propriedades:

(a) $M_{\psi_{\mathbb{J}}}(\lambda)$ é um $\mathfrak{U}\left(\mathfrak{g}_{-S_{\psi_{J}}}\right)$-módulo livre de posto 1 .

(b) $M_{\psi_{J}}(\lambda)$ tem um único submódulo maximal e consequentemente um único quociente irredutível.

(c) $P\left(M_{\psi_{\mathbb{J}}}(\lambda)\right)=\bigcup_{\beta \in \dot{Q}_{+}}\{\lambda-\beta+n \delta \mid n \in \mathbb{Z}\}$, em que $\dot{Q}_{+}$é o monóide abeliano livre gerado por todas as raízes simples da base $\Pi$ de $\dot{\Delta}_{+}$.

(d) Se $\psi_{\mathbb{J}}(k) \neq \psi_{\mathbb{J}}(l)$ para alguns $k, l \in \mathbb{N}$, então $\operatorname{dim} M_{\psi_{\mathbb{J}}}(\lambda)_{\mu}=\infty$, para qualquer $\mu \in P\left(M_{\psi_{\mathbb{J}}}(\lambda)\right)$.

Em seguida, temos um critério de irredutibilidade:

Teorema 2.5.4 (([2]), Teorema 3.5). Seja $\lambda \in \mathfrak{h}^{*}$ tal que $\lambda(c)=a$. Então, $M_{\psi_{\mathbb{J}}}(\lambda)$ é irredutivel se e somente se $a \neq 0$.

\subsubsection{Realização de Campos Livres de $\mathfrak{s l}(2, \mathbb{C})$}

Nesta subseção, seguindo [16], estudaremos a construção de uma realização $\rho_{\mathbb{J}}$ dos módulos de Verma $\mathbb{J}$-imaginários para $\hat{\mathfrak{s l}}(2, \mathbb{C})$. Inicialmente, será construída uma candidata $\varphi_{\mathbb{J}}$, que preserva o colchete de Lie, mas não está bem definida. Em seguida, será efetuada a correção do problema, através da aplicação de duas convenientes anti-involuções.

Notemos que aqui, como $1 \leq i, j \leq 2$, podemos usar a notação $E_{m}, F_{m}, H_{m}$, ao invés de $E_{1 m}, F_{1 m}, H_{1 m}$, bem como $x_{m}, y_{m}$, ao invés de $x_{11, m}, y_{1 m}$, etc., com $m \in \mathbb{Z}$.

Definindo $\mathbb{C}[\mathbf{x}, \mathbf{y}]=\mathbb{C}[\mathbf{x}] \otimes_{\mathbb{C}} \mathbb{C}[\mathbf{y}]$, consideremos a função $\varphi_{\mathbb{J}}: \hat{\mathfrak{s l}}(2, \mathbb{C}) \longrightarrow \mathfrak{g l}(\mathbb{C}[\mathbf{x}, \mathbf{y}])$ dada por:

$$
\begin{aligned}
\varphi_{\mathbb{J}}\left(E_{m}\right) & =-\frac{\partial}{\partial_{x_{m}}}, \\
\varphi_{\mathbb{J}}\left(F_{m}\right) & =\sum_{l, j \in \mathbb{Z}} x_{l} x_{j-l-m} \frac{\partial}{\partial_{x_{j}}}+\sum_{j \in \mathbb{J}} x_{j-m} \frac{\partial}{\partial_{y_{j}}}+2 K \sum_{j \in \mathbb{J}} j y_{j} x_{-j-m}+K m x_{-m}-2 J x_{-m}, \\
\varphi_{\mathbb{J}}\left(H_{m}\right) & =-2 \sum_{j \in \mathbb{Z}} x_{j-m} \frac{\partial}{\partial_{x_{j}}}-\psi_{\mathbb{J}}(m) \frac{\partial}{\partial_{y_{m}}}+\psi_{\mathbb{J}}(-m) 2 m K y_{-m}+2 \delta_{m, 0} J .
\end{aligned}
$$

Em seguida, mostraremos que o colchete de Lie é preservado por essas relações. Para todo $m, n \in \mathbb{Z}$, temos: 


$$
\begin{aligned}
& {\left[\varphi_{\mathbb{J}}\left(H_{m}\right), \varphi_{\mathbb{J}}\left(E_{n}\right)\right]=\left[-2 \sum_{j \in \mathbb{Z}} x_{j-m} \frac{\partial}{\partial_{x_{j}}}-\psi_{\mathbb{J}}(m) \frac{\partial}{\partial_{y_{m}}}+\psi_{\mathbb{J}}(-m) 2 m K y_{-m}+2 \delta_{m, 0} J,-\frac{\partial}{\partial_{x_{n}}}\right]} \\
& =2\left[\sum_{j \in \mathbb{Z}} x_{j-m} \frac{\partial}{\partial_{x_{j}}}, \frac{\partial}{\partial_{x_{n}}}\right]=-2 \frac{\partial}{\partial_{x_{m+n}}}=2 \varphi_{\mathbb{J}}\left(E_{m+n}\right)=\varphi_{\mathbb{J}}\left(\left[H_{m}, E_{n}\right]\right) ; \\
& {\left[\varphi_{\mathbb{J}}\left(H_{m}\right), \varphi_{\mathbb{J}}\left(H_{n}\right)\right]=\left[-2 \sum_{j \in \mathbb{Z}} x_{j-m} \frac{\partial}{\partial_{x_{j}}}-\psi_{\mathbb{J}}(m) \frac{\partial}{\partial_{y_{m}}}+\psi_{\mathbb{J}}(-m) 2 m K y_{-m}+2 \delta_{m, 0} J,\right.} \\
& \left.-2 \sum_{i \in \mathbb{Z}} x_{i-n} \frac{\partial}{\partial_{x_{i}}}-\psi_{\mathbb{J}}(n) \frac{\partial}{\partial_{y_{n}}}+\psi_{\mathbb{J}}(-n) 2 n K y_{-n}+2 \delta_{n, 0} J\right] \\
& =4\left[\sum_{j \in \mathbb{Z}} x_{j-m} \frac{\partial}{\partial_{x_{j}}}, \sum_{i \in \mathbb{Z}} x_{i-n} \frac{\partial}{\partial_{x_{i}}}\right]-\left[\psi_{\mathbb{J}}(m) \frac{\partial}{\partial_{y_{m}}}, \psi_{\mathbb{J}}(-n) 2 n K y_{-n}\right] \\
& -\left[\psi_{\mathbb{J}}(-m) 2 m K y_{-m}, \psi_{\mathbb{J}}(n) \frac{\partial}{\partial_{y_{n}}}\right] \\
& { }^{(\star)} \underbrace{4 \sum_{j \in \mathbb{Z}} x_{j-m} \frac{\partial}{\partial_{x_{j+n}}}-4 \sum_{i \in \mathbb{Z}} x_{i-n} \frac{\partial}{\partial_{x_{i+m}}}}_{0}-2 \delta_{m,-n} \psi_{\mathbb{J}}(m) \psi_{\mathbb{J}}(-n) n K+2 \delta_{m,-n} \psi_{\mathbb{J}}(n) \psi_{\mathbb{J}}(-m) m K \\
& =2 \delta_{m,-n} m K\left(\psi_{\mathbb{J}}(m) \psi_{\mathbb{J}}(-n)+\psi_{\mathbb{J}}(n) \psi_{\mathbb{J}}(-m)\right) \\
& ={ }^{(\star \star)} 2 \delta_{m,-n} m K \underbrace{\left(\psi_{\mathbb{J}}(m) \psi_{\mathbb{J}}(m)+\psi_{\mathbb{J}}(n) \psi_{\mathbb{J}}(n)\right)}_{1}=\varphi_{\mathbb{J}}\left(\left[H_{m}, H_{n}\right]\right) \text {; }
\end{aligned}
$$

$(\star):$ Fazendo $k=j+n$ em $4 \sum_{j \in \mathbb{Z}} x_{j-m} \frac{\partial}{\partial_{x_{j+n}}}$ e $k=i+m$ em $-4 \sum_{i \in \mathbb{Z}} x_{i-n} \frac{\partial}{\partial_{x_{i+m}}}$

$(\star \star)$ :Se $m=n=0$, o colchete está naturalmente preservado, pois $\left[H_{0}, H_{0}\right]=0$. Suponhamos então que $m \neq 0$. Assim, se $\psi_{\mathbb{J}}(m)=0$, ou $m \in \mathbb{N}^{*} \backslash \mathbb{J}$ ou $-m \in \mathbb{J}$. Em ambos os casos, $\psi_{\mathbb{J}}(-m)=1$. O mesmo raciocínio pode ser usado se $\psi_{\mathbb{J}}(m)=1$. 


$$
\begin{aligned}
{\left[\varphi_{\mathbb{J}}\left(H_{m}\right), \varphi_{\mathbb{J}}\left(F_{n}\right)\right] } & =\left[-2 \sum_{j \in \mathbb{Z}} x_{j-m} \frac{\partial}{\partial_{x_{j}}}-\psi_{\mathbb{J}}(m) \frac{\partial}{\partial_{y_{m}}}+\psi_{\mathbb{J}}(-m) 2 m K y_{-m}+2 \delta_{m, 0} J,\right. \\
& \left.\sum_{l, i \in \mathbb{Z}} x_{l} x_{i-l-n} \frac{\partial}{\partial_{x_{i}}}+\sum_{i \in \mathbb{J}} x_{i-n} \frac{\partial}{\partial_{y_{i}}}+2 K \sum_{i \in \mathbb{J}} i y_{i} x_{-i-n}+K n x_{-n}-2 J x_{-n}\right] \\
& =-2\left[\sum_{j \in \mathbb{Z}} x_{j-m} \frac{\partial}{\partial_{x_{j}}}, \sum_{l, i \in \mathbb{Z}} x_{l} x_{i-l-n} \frac{\partial}{\partial_{x_{i}}}\right]-2\left[\sum_{j \in \mathbb{Z}} x_{j-m} \frac{\partial}{\partial_{x_{j}}}, \sum_{i \in \mathbb{J}} x_{i-n} \frac{\partial}{\partial_{y_{i}}}\right] \\
& \left.\left.-4 K\left[\sum_{j \in \mathbb{Z}} x_{j-m} \frac{\partial}{\partial_{x_{j}}}, \sum_{i \in \mathbb{J}} i y_{i} x_{-i-n}\right]-2 K\left[\sum_{j \in \mathbb{Z}} x_{j-m} \frac{\partial}{\partial_{x_{j}}}, n x_{-n}\right]+4 J\right] \sum_{j \in \mathbb{Z}} x_{j-m} \frac{\partial}{\partial_{x_{j}}}, x_{-n}\right] \\
& -2 K\left[\psi_{\mathbb{J}}(m) \frac{\partial}{\partial_{y_{m}}}, \sum_{i \in \mathbb{J}} i y_{i} x_{-i-n}\right]+\left[\psi_{\mathbb{J}}(-m) 2 m K y_{-m}, \sum_{i \in \mathbb{J}} x_{i-n} \frac{\partial}{\partial_{y_{i}}}\right] \\
& =-2 \sum_{j \in \mathbb{Z}} x_{j-m} \sum_{i \in \mathbb{Z}} x_{i-j-n} \frac{\partial}{\partial_{x_{i}}}-2 \sum_{j \in \mathbb{Z}} x_{j-m} \sum_{l \in \mathbb{Z}} x_{l} \frac{\partial}{\partial_{x_{j+l+n}}}+2 \sum_{l, i \in \mathbb{Z}} x_{l} x_{i-l-n} \frac{\partial}{\partial_{x_{i+m}}} \\
& -2 \sum_{j \in \mathbb{Z}} x_{j-m} \psi_{\mathbb{J}}(j+n) \frac{\partial}{\partial_{y_{j+n}}}-4 K \sum_{j \in \mathbb{Z}} x_{j-m} \psi_{\mathbb{J}}(-j-n)(-j-n) y_{-j-n}-2 K n x_{-n-m} \\
& +4 J x_{-n-m}-2 K \psi_{\mathbb{J}}(m) \psi_{\mathbb{J}}(m) m x_{-m-n}-2 K \psi_{\mathbb{J}}(-m) \psi_{\mathbb{J}}(-m) m x_{-m-n} \\
& ={ }^{(\star)} 2 \sum_{j, l \in \mathbb{Z}} x_{l} x_{j-m-l-n} \frac{\partial}{\partial_{x_{j}}}-4 \sum_{k, i \in \mathbb{Z}} x_{k} x_{i-k-m-n} \frac{\partial}{\partial_{x_{i}}}-2 \sum_{i \in \mathbb{Z}} x_{i-n-m} \psi_{\mathbb{J}}(i) \frac{\partial}{\partial_{y_{i}}} \\
& -4 K \sum_{k \in \mathbb{Z}} x_{k-n-m} \psi_{\mathbb{J}}(-k)(-k) y_{-k}-2 K n x_{-n-m}+4 J x_{-n-m}-2 K m x_{-m-n} \\
& ={ }^{(\star \star)}-2 \sum_{j, l \in \mathbb{Z}} x_{l} x_{j-m-l} \frac{\partial}{\partial_{x_{j}}}-2 \sum_{i \in \mathbb{J}} x_{i-n-m} \frac{\partial}{\partial_{y_{i}}}-4 K \sum_{l \in \mathbb{J}} x_{-l-m-n} l y_{l} \\
& -2 K(m+n) x_{-m-n}+4 J x_{-n-m}=-2 \varphi_{\mathbb{J}}\left(F_{m+n}\right) \\
& =\varphi_{\mathbb{J}}\left(\left[H_{m}, F_{n}\right]\right) ;
\end{aligned}
$$

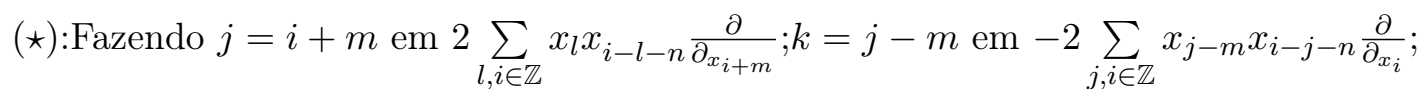

$k=j+l+n$ em $-2 \sum_{j, l \in \mathbb{Z}} x_{j-m} x_{l} \frac{\partial}{\partial_{x_{j+l+n}}} ; i=j+n$ em $-2 \sum_{j \in \mathbb{Z}} x_{j-m} \psi_{\mathbb{J}}(j+n) \frac{\partial}{\partial y_{j+n}} ;$

$k=j+n \mathrm{em}-4 K \sum_{j \in \mathbb{Z}} x_{j-m} \psi_{\mathbb{J}}(-j-n)(-j-n) y_{-j-n} ;$

$(\star \star)$ :Fazendo $l=-k$ em $-4 K \sum_{k \in \mathbb{Z}} x_{k-n-m} \psi_{\mathbb{J}}(-k)(-k) y_{-k}$ 


$$
\begin{aligned}
{\left[\varphi_{\mathbb{J}}\left(E_{m}\right), \varphi_{\mathbb{J}}\left(F_{n}\right)\right] } & =\left[-\frac{\partial}{\partial_{x_{m}}}, \sum_{l, j \in \mathbb{Z}} x_{l} x_{j-l-n} \frac{\partial}{\partial_{x_{j}}}+\sum_{j \in \mathbb{J}} x_{j-n} \frac{\partial}{\partial_{y_{j}}}+2 K \sum_{j \in \mathbb{J}} j y_{j} x_{-j-n}+K n x_{-n}-2 J x_{-n}\right] \\
& =-\left[\frac{\partial}{\partial_{x_{m}}}, \sum_{l, j \in \mathbb{Z}} x_{l} x_{j-l-n} \frac{\partial}{\partial_{x_{j}}}\right]-\left[\frac{\partial}{\partial_{x_{m}}}, \sum_{j \in \mathbb{J}} x_{j-n} \frac{\partial}{\partial_{y_{j}}}\right]-2 K\left[\frac{\partial}{\partial_{x_{m}}}, \sum_{j \in \mathbb{J}} j y_{j} x_{-j-n}\right] \\
& -K\left[\frac{\partial}{\partial_{x_{m}}}, n x_{-n}\right]+2 J\left[\frac{\partial}{\partial_{x_{m}}}, x_{-n}\right] \\
& =-\sum_{j \in \mathbb{Z}} x_{j-m-n} \frac{\partial}{\partial_{x_{j}}}-\sum_{l \in \mathbb{Z}} x_{l} \frac{\partial}{\partial_{x_{m+l+n}}}-\psi_{\mathbb{J}}(m+n) \frac{\partial}{\partial_{y_{m+n}}}+2 K(m+n) \psi_{\mathbb{J}}(-m-n) y_{-m-n} \\
& +\delta_{m,-n}(K m+2 J) \\
& ={ }^{(\star)}-2 \sum_{j \in \mathbb{Z}} x_{j-m-n} \frac{\partial}{\partial_{x_{j}}}-\psi_{\mathbb{J}}(m+n) \frac{\partial}{\partial_{y_{m+n}}}+2 \psi_{\mathbb{J}}(-m-n)(m+n) K y_{-m-n}+2 \delta_{m,-n} J+K m \delta_{m,-n} \\
& =\varphi_{\mathbb{J}}\left(H_{m+n}\right)+K m \delta_{m,-n}=\varphi_{\mathbb{J}}\left(\left[E_{m}, F_{n}\right]\right) .
\end{aligned}
$$

$(\star)$ :Fazendo $j=l+m+n$ em $-\sum_{l \in \mathbb{Z}} x_{l} \frac{\partial}{\partial_{x_{l+m+n}}}$.

Notemos porém que $\varphi_{\mathbb{J}}\left(F_{m}\right)$ não está bem definida, pois se $\mathbb{J} \subset \mathbb{N}^{*}$ for um conjunto infinito, ao aplicarmos esse operador no 1 , teremos uma soma infinita. A correção do problema é feita através da utilização de duas anti-involuções:

$\rho_{1}: \hat{\mathfrak{s l}}(2, \mathbb{C}) \longrightarrow \hat{\mathfrak{s l}}(2, \mathbb{C})$ dada por

$$
\rho_{1}\left(E_{m}\right)=-F_{-m}, \rho_{1}\left(F_{m}\right)=-E_{-m}, \rho_{1}\left(H_{m}\right)=H_{-m} \text { e } \rho_{1}(K)=K,
$$

e $\rho_{2}: \mathfrak{g l}(\mathbb{C}[\mathbf{x}, \mathbf{y}]) \longrightarrow \mathfrak{g l}(\mathbb{C}[\mathbf{x}, \mathbf{y}])$ dada por

$$
\rho_{2}\left(x_{-m}\right)=\frac{\partial}{\partial_{x_{m}}}, \rho_{2}\left(\frac{\partial}{\partial_{x_{m}}}\right)=x_{-m}, \rho_{2}\left(y_{k}\right)=-\frac{\partial}{\partial_{y_{k}}} \text { e } \rho_{2}\left(\frac{\partial}{\partial_{y_{k}}}\right)=-y_{k} .
$$

Definimos então $\rho_{\mathbb{J}}: \hat{\mathfrak{s l}}(2, \mathbb{C}) \longrightarrow \mathfrak{g l}(\mathbb{C}[\mathbf{x}, \mathbf{y}])$ como $\rho_{\mathbb{J}}:=\rho_{2} \circ \varphi_{\mathbb{J}} \circ \rho_{1}$, e teremos o homomorfismo desejado:

$$
\begin{aligned}
\rho_{\mathbb{I}}\left(F_{m}\right) & =x_{m}, \\
\rho_{\mathbb{J}}\left(E_{m}\right) & =-\sum_{l, j \in \mathbb{Z}} x_{j+l+m} \frac{\partial}{\partial_{x_{l}}} \frac{\partial}{\partial_{x_{j}}}+\sum_{j \in \mathbb{J}} y_{j} \frac{\partial}{\partial_{x_{-j-m}}}+2 K \sum_{j \in \mathbb{J}} j \frac{\partial}{\partial_{y_{j}}} \frac{\partial}{\partial_{x_{j-m}}}+(K m+2 J) \frac{\partial}{\partial_{x_{-m}}}, \\
\rho_{\mathbb{J}}\left(H_{m}\right) & =-2 \sum_{j \in \mathbb{Z}} x_{j+m} \frac{\partial}{\partial_{x_{j}}}+\psi_{\mathbb{J}}(-m) y_{-m}+\psi_{\mathbb{J}}(m) 2 m K \frac{\partial}{\partial_{y_{m}}}+2 \delta_{m, 0} J .
\end{aligned}
$$

Trata-se realmente de uma representação, pois $\varphi_{\mathbb{J}}$ já preservava o colchete de Lie, e estamos agora apenas compondo com duas anti-involuções.

Observação 2.5.5. Para o caso em que $n>2$, ainda em [16], é construída a realização de campos livres $\rho_{\mathbb{J}}$ para $\hat{\mathfrak{s l}}(n, \mathbb{C})$, utilizando a linguagem de distribuições formais. 
A realização é dada pela função $\rho_{\mathbb{J}}: \hat{\mathfrak{s} l}(n, \mathbb{C}) \longrightarrow \mathfrak{g l}(\mathbb{C}[\mathbf{x}, \mathbf{y}])$ da seguinte forma:

$$
\begin{aligned}
\rho_{\mathbb{J}}\left(F_{r m}\right)= & a_{r, r+1, m}+\sum_{j=1}^{r-1} \sum_{l \in \mathbb{Z}} a_{j, r+1, m-l} a_{j, r, l}^{*}, \\
\rho_{\mathbb{J}}\left(E_{r m}\right)= & \sum_{l_{1}, l_{2} \in \mathbb{Z}} a_{r, r+1, m-l_{1}-l_{2}} a_{r, r+1, l_{1}}^{*} a_{r, r+1, l_{2}}^{*}+\sum_{l \in \mathbb{Z}}\left(-\sum_{j=r+2}^{n} a_{r+1, j, m-l} a_{r+1, j, l}^{*}\right) \\
& +\sum_{l_{1}, l_{2} \in \mathbb{Z}} a_{r, j, m-l_{1}-l_{2}} a_{r, j, l_{1}}^{*} a_{r, j, l_{2}}^{*}-\sum_{l_{1}, l_{2} \in \mathbb{Z}} a_{r+1, j, m-l_{1}-l_{2}} a_{r+1, j, l_{1}}^{*} a_{r, r+1, l_{2}}^{*} \\
& -\gamma \sum_{l \in \mathbb{Z}}\left(a_{r, r+1, l}^{*} b_{r, m-l}+\frac{1}{2} \delta_{m<0}\left(a_{r, r+1, l}^{*} b_{r-1, m-l}+a_{r, r+1, l}^{*} b_{r+1, m-l}\right)\right) \\
& -\frac{\gamma}{2} a_{r, r+1, m+1}^{*}, \\
\rho_{\mathbb{J}}\left(H_{r m}\right)= & \sum_{l \in \mathbb{Z}}\left(2 a_{r, r+1, m-l} a_{r, r+1, l}^{*}+\sum_{i=1}^{r-1} a_{i, r+1, m-l} a_{i, r+1, l}^{*}\right. \\
& -\sum_{i=1}^{r-1} a_{i, r, m-l} a_{i, r, l}^{*}+\sum_{j=r+2}^{n} a_{r, j, m-l} a_{r, j, l}^{*}-\sum_{j=r+2}^{n} a_{r+1, j, m-l} a_{r+1, j, n}^{*} \\
& \left.-\gamma b_{r m}+\delta_{m<0} \frac{\gamma}{2}\left(b_{r-1, m}+b_{r+1, m}\right)\right) .
\end{aligned}
$$




\section{Capítulo 3}

\section{Realização de Campos Livres}

Muitas vezes, ao buscarmos representar uma álgebra de Lie em um espaço vetorial, queremos que o mesmo seja um espaço de polinômios. Na verdade, queremos encontrar "cópias"dessa álgebra em anéis de operadores diferenciais sobre algum espaço vetorial de polinômios, pois os elementos desses anéis são relativamente fáceis de serem manuseados.

Assim, este capítulo é dedicado à introdução do conceito de realização de campos livres, que é um tipo de representação em algum espaço de polinômios, com destaque para a primeira realização de campos livres, passando em seguida rapidamente pela segunda realização.

Não iremos nos ater à construção propriamente dita, que utiliza tanto conceitos e resultados de Variedades e Grupos de Lie, como de Geometria Algébrica, tendo em vista que não é o foco deste trabalho, e os detalhes podem ser consultados em [5] e [6]. Nossa abordagem do assunto será através da exibição de exemplos, que ilustram de forma eficiente o que precisamos, dentro do contexto das álgebras de Lie afim.

Em primeiro lugar, estudaremos um exemplo de representação em que a álgebra de Lie é simples de dimensão finita, o que servirá como modelo para o caso em que é uma álgebra de Kac-Moody do tipo afim, dando origem à primeira realização.

\subsection{Caso de Dimensão Finita}

Seja $\dot{\mathfrak{g}}$ uma álgebra de Lie simples de dimensão finita, com decomposição triangular $\dot{\mathfrak{n}}_{+} \oplus \dot{\mathfrak{h}} \oplus \dot{\mathfrak{n}}_{-}$, em que $\dot{\mathfrak{h}}$ é a subálgebra de Cartan de $\dot{\mathfrak{g}}$. A construção efetuada nas referências citadas fornece-nos uma representação de $\dot{\mathfrak{g}}$ no espaço dos polinômios em $m=\left|\Delta_{+}\right|$variáveis. Na verdade, temos um homomorfismo de álgebras de Lie de $\dot{\mathfrak{g}}$ na álgebra de Weyl em $m$ variáveis $\mathcal{A}_{m}$, em que $\mathcal{A}_{m}$ é a álgebra não comutativa gerada pelos operadores multiplicação $x_{1}, \ldots, x_{m}$ e pelas derivações $\frac{\partial}{\partial x_{i}}$, com $i \in\{1, \ldots, m\}$ nesse espaço de polinômios.

Além disso, o espaço de polinômios em $m$ variáveis acima citado é isomorfo a $M^{*}(0)$, como $\dot{\mathfrak{g}}$-módulo, em que $M^{*}(0)$ é o módulo contragradiente ao módulo de Verma $M(0)$ sobre $\dot{\mathfrak{g}}$, com peso máximo 0 .

Destacamos que o módulo contragradiente que estamos denotando por $M^{*}(0)$ é o módulo dual restrito de $M(0)$, com respeito à decomposição de peso induzida pela ação de $\mathfrak{h}$, já que $M(0)$ possui uma decomposição em espaços de peso, em que cada um desses espaços possui dimensão finita, e a ação de $\dot{\mathfrak{g}}$ em $M^{*}(0)$ é dada por 
$(x f)(v)=f(\dot{\omega}(x) v)$, para todo $v \in M(0), x \in \dot{\mathfrak{g}}$ e $f \in M^{*}(0)$,

em que $\dot{\omega}$ é uma anti-involução em $\dot{\mathfrak{g}}$ que preserva $\mathfrak{h}$ e permuta os geradores de Chevalley $e_{i}$ e $f_{i}$.

Dito de outra forma, se $M(0)=\bigoplus_{\mu \in \dot{\mathfrak{h}}^{*}} M_{\mu}$ é a decomposição em espaços de peso do módulo de Verma $M(0)$, então $M^{*}(0)=\bigoplus_{\mu \in \dot{\mathfrak{h}}^{*}} M_{\mu}^{*}$ é o módulo contragradiente.

Exemplo 3.1.1. Para o primeiro exemplo, naturalmente, tomaremos $\dot{\mathfrak{g}}=\mathfrak{s l}(2, \mathbb{C})$, com a base padrão

$$
e=\left(\begin{array}{ll}
0 & 1 \\
0 & 0
\end{array}\right), h=\left(\begin{array}{cc}
1 & 0 \\
0 & -1
\end{array}\right), f=\left(\begin{array}{ll}
0 & 0 \\
1 & 0
\end{array}\right),
$$

e colchete de Lie dado por $[e, f]=h,[h, e]=2 e$ e $[h, f]=-2 f$. Após efetuada a construção como em [6], será obtida uma representação $\rho: \dot{\mathfrak{g}} \longrightarrow \mathfrak{g l}(\mathbb{C}[x])$ no espaço $\mathbb{C}[x]$ dada por:

$$
e \longmapsto \frac{d}{d x}, h \longmapsto-2 x \frac{d}{d x}, f \longmapsto-x^{2} \frac{d}{d x} \text {. }
$$

Para mostrar que $\rho$ é de fato um homomorfismo de álgebras de Lie, basta analisar o que ocorre em um monômio $x^{m} \in \mathbb{C}[x], m \in \mathbb{Z}_{+}$. Temos então

$$
\begin{aligned}
& {[\rho(e), \rho(f)]\left(x^{m}\right)=(-m(m+1)+m(m-1)) x^{m}=-2 m x^{m}=\rho(h)\left(x^{m}\right)=\rho([e, f])\left(x^{m}\right) ;} \\
& {[\rho(h), \rho(e)]\left(x^{m}\right)=\left(-2 m(m-1)+2 m^{2}\right) x^{m-1}=2 m x^{m-1}=\rho(2 e)\left(x^{m}\right)=\rho([h, e])\left(x^{m}\right) ;} \\
& {[\rho(h), \rho(f)]\left(x^{m}\right)=\left(2 m(m+1)-2 m^{2}\right) x^{m+1}=2 m x^{m+1}=\rho(-2 f)\left(x^{m}\right)=\rho([h, f])\left(x^{m}\right) .}
\end{aligned}
$$

Ainda conforme [6], teremos $\mathbb{C}[x] \cong M^{*}(0)$, como $\dot{\mathfrak{g}}$-módulos.

Observação 3.1.2. Considerando ainda $\dot{\mathfrak{g}}=\mathfrak{s l}(2, \mathbb{C})$, mas já antecipando o que será necessário no caso de uma álgebra de Kac-Moody afim, podemos tomar outro caminho para obter uma representação $\theta: \dot{\mathfrak{g}} \longrightarrow \mathfrak{g l}(\mathbb{C}[x])$, aplicando a $\dot{\mathfrak{g}}$ uma composição de duas anti-involuções:

$$
e \leftrightarrow f, h \leftrightarrow h ; x \leftrightarrow \frac{d}{d x}
$$

Teremos a seguinte realização do módulo de Verma $M(0)$ em operadores diferenciais de segunda ordem:

$$
e \longmapsto-x\left(\frac{d}{d x}\right)^{2}, h \longmapsto-2 x \frac{d}{d x}, f \longmapsto x
$$

Como o colchete de Lie já estava preservado anteriormente, a aplicação das duas anti-involuções fornece ainda um homomorfismo. 


\subsection{Caso Afim}

Como feito anteriormente no caso de dimensão finita, faremos apenas algumas considerações sobre o caso geral, e trataremos de um exemplo específico. No caso das álgebras de Kac-Moody do tipo afim, esse caso específico será a álgebra $\hat{\mathfrak{s l}}(2, \mathbb{C})$.

No caso atual, ao serem efetuadas construções análogas às que foram efetuadas para o caso de dimensão finita, seguindo [6], iremos obter uma representação da álgebra de Kac-Moody afim em um espaço vetorial de polinômios em infinitas variáveis, em que teremos uma ação da mesma através de operadores diferenciais, o que fornece uma realização para o módulo contragradiente ao módulo de Verma $M(0)$.

Destacamos que, no caso atual, não é possível obter módulos de Verma padrão a partir dessa construção, o que é uma importante diferença entre este e o caso de dimensão finita.

Exemplo 3.2.1. Para ilustrar o caso de uma álgebra de Kac-Moody afim, tomaremos $\dot{\mathfrak{g}}=\mathfrak{s l}(2, \mathbb{C})$ como sendo a álgebra de Lie simples de dimensão finita a partir da qual obtemos $\widehat{\mathcal{L}}(\dot{\mathfrak{g}})$, que denotaremos por $\hat{\mathfrak{s l}}(2, \mathbb{C})$. Mantendo a notação usual, temos que os elementos

$$
e_{n}=e \otimes t^{n}, h_{n}=h \otimes t^{n}, f_{n}=f \otimes t^{n}, n \in \mathbb{Z},
$$

em que $e, f, h$ formam uma base de $\mathfrak{s l}(2, \mathbb{C})$, juntamente com o elemento central $c$ e a derivação $d$ formam uma base de $\hat{\mathfrak{s l}}(2, \mathbb{C})$.

Novamente seguindo [6], após efetuada a construção geométrica, iremos obter uma candidata $\rho: \hat{\mathfrak{s l}}(2, \mathbb{C}) \longrightarrow \mathfrak{g l}\left(\mathbb{C}\left[x_{m}, m \in \mathbb{Z}\right]\right)$ à representação de $\hat{\mathfrak{s} l}(2, \mathbb{C})$ no anel de polinômios $\mathbb{C}\left[x_{m}, m \in \mathbb{Z}\right]$, como segue:

$$
e_{n} \longmapsto \frac{\partial}{\partial x_{n}}, h_{n} \longmapsto-2 \sum_{m \in \mathbb{Z}} x_{m} \frac{\partial}{\partial x_{n+m}}, f_{n} \longmapsto-\sum_{m, k \in \mathbb{Z}} x_{m} x_{k} \frac{\partial}{\partial x_{n+m+k}}
$$

Para simplificar a notação, denotaremos $\frac{\partial}{\partial x_{j}}$ apenas por $\partial_{x_{j}}$, para todo $j \in \mathbb{Z}$.

É possível verificar que o colchete de Lie é preservado através desses operadores, sendo suficiente analisar em um monômio $x_{m_{1}}^{\alpha_{1}} \ldots x_{m_{l}}^{\alpha_{l}} \in \mathbb{C}\left[x_{m}, m \in \mathbb{Z}\right], \alpha_{i} \in \mathbb{Z}_{+}$, em que $i \in\{1, \ldots, l\}$.

De fato, para todo $n_{1}, n_{2} \in \mathbb{Z}$, temos: 


$$
\begin{aligned}
{\left[\rho\left(h_{n_{1}}\right), \rho\left(e_{n_{2}}\right)\right] } & =\left(-2 \sum_{m \in \mathbb{Z}} x_{m} \partial_{x_{n_{1}+m}}\right) \partial_{x_{n_{2}}}+\partial_{x_{n_{2}}}\left(2 \sum_{m \in \mathbb{Z}} x_{m} \partial_{x_{n_{1}+m}}\right) \\
& \left.=2 \partial_{x_{n_{1}+n_{2}}}\right) \\
& =\rho\left(2 e_{n_{1}+n_{2}}\right)=\rho\left(\left[h_{n_{1}}, e_{n_{2}}\right]\right) ; \\
{\left[\rho\left(h_{n_{1}}\right), \rho\left(f_{n_{2}}\right)\right] } & =\left(2 \sum_{j \in \mathbb{Z}} x_{j} \partial_{x_{n_{1}+j}}\right)\left(\sum_{m, k \in \mathbb{Z}} x_{m} x_{k} \partial_{x_{n_{2}+m+k}}\right) \\
& -\left(\sum_{m, k \in \mathbb{Z}} x_{m} x_{k} \partial_{x_{n_{2}+m+k}}\right)\left(2 \sum_{j \in \mathbb{Z}} x_{j} \partial_{x_{n_{1}+j}}\right) \\
& =2 \sum_{j \in \mathbb{Z}} x_{j}\left(\sum_{m \in \mathbb{Z}} x_{m} \partial_{x_{n_{2}}+m+n_{1}+j}+\sum_{k \in \mathbb{Z}} x_{k} \partial_{x_{n_{2}+n_{1}+j+k}}\right)-2 \sum_{m, k \in \mathbb{Z}} x_{m} x_{k} \partial_{x_{n_{1}+n_{2}+m+k}} \\
& =2 \sum_{j, m \in \mathbb{Z}} x_{j} x_{m} \partial_{x_{n_{2}+m+n_{1}+j}} \\
& =\rho\left(-2 f_{n_{1}+n_{2}}\right)=\rho\left(\left[h_{n_{1}}, f_{n_{2}}\right]\right) ; \\
{\left[\rho\left(e_{n_{1}}\right), \rho\left(f_{n_{2}}\right)\right] } & =-\partial_{x_{n_{1}}}\left(\sum_{m, k \in \mathbb{Z}} x_{m} x_{k} \partial_{x_{n_{2}+m+k}}\right)+\left(\sum_{m, k \in \mathbb{Z}} x_{m} x_{k} \partial_{x_{n_{2}+m+k}}\right) \partial_{x_{n_{1}}} \\
= & -\sum_{m \in \mathbb{Z}} x_{m} \partial_{x_{n_{2}+m+n_{1}}}-\sum_{k \in \mathbb{Z}} x_{k} \partial_{x_{n_{2}+n_{1}+k}} \\
= & -2 \sum_{m \in \mathbb{Z}} x_{m} \partial_{x_{n_{2}+n_{1}+m}} \\
= & \rho\left(h_{n_{1}+n_{2}}\right)=\rho\left(\left[e_{n_{1}}, f_{n_{2}}\right]\right) . \\
&
\end{aligned}
$$

Notemos que ainda não temos uma representação em $\mathbb{C}\left[x_{m}, m \in \mathbb{Z}\right]$, pois o operador correspondente a $f_{n}$ não está bem definido, já que aplicado sucessivamente a elementos do espaço $\mathbb{C}\left[x_{m}, m \in \mathbb{Z}\right]$ produzirá uma soma infinita de monômios. Um caminho para que esse fato seja corrigido é a aplicação das anti-involuções:

$$
e_{n} \leftrightarrow f_{n}, h \leftrightarrow h ; x_{n} \leftrightarrow \partial x_{n},
$$

que nos fornecerá:

$$
f_{n} \longmapsto x_{n}, h_{n} \longmapsto-2 \sum_{m \in \mathbb{Z}} x_{n+m} \partial_{x_{m}}, e_{n} \longmapsto-\sum_{m, k \in \mathbb{Z}} x_{n+m+k} \partial_{x_{m}} \partial_{x_{k}}
$$

Tendo em vista que o colchete de Lie já era preservado por $\rho$, mesmo antes da aplicação das anti-involuções, ele continua preservado pelas novas fórmulas.

As fórmulas (3.1) definem a primeira realização de campos livres de $\hat{\mathfrak{s} l}(2, \mathbb{C})$ no anel de polinômios $\mathbb{C}\left[x_{m}, m \in \mathbb{Z}\right]$. Este módulo é um quociente do módulo de Verma imaginário de peso máximo 0 por um submódulo gerado pelos elementos $h_{n} \otimes 1, n<0$. 
Destacamos que estamos considerando o elemento central $c$ e $h_{0}$ agindo no módulo $\mathbb{C}\left[x_{m}, m \in \mathbb{Z}\right]$ como multiplicação por 0 . A ação da derivação $d$ pode ser a multiplicação por um escalar qualquer $\mu \in \mathbb{C}$.

Bernard e Felder utilizaram a construção de Borel-Weyl (que não foi estudada neste trabalho), para obter uma realização do módulo de Verma imaginário com uma ação não trivial do elemento central.

A partir dessa construção, com a aplicação das anti-involuções:

$$
\begin{array}{r}
e_{n} \leftrightarrow-f_{-n}, h_{n} \leftrightarrow h_{-n}, c \leftrightarrow c \mathrm{e} \\
x_{-n} \leftrightarrow \partial_{x_{n}}, y_{k} \leftrightarrow-\partial_{y_{k}},
\end{array}
$$

é obtida a seguinte representação $\rho: \hat{\mathfrak{s l}}(2, \mathbb{C}) \longrightarrow \mathfrak{g l}\left(\mathbb{C}\left[x_{m}, m \in \mathbb{Z}\right] \otimes \mathbb{C}\left[y_{n}, n>0\right]\right)$ de $\hat{\mathfrak{s l}}(2, \mathbb{C})$

$$
\begin{aligned}
& f_{n} \longmapsto x_{n}, h_{n} \longmapsto-2 \sum_{m \in \mathbb{Z}} x_{m+n} \partial_{x_{m}}+\delta_{n<0} y_{-n}+\delta_{n>0} 2 n K \partial_{y_{n}}+\delta_{n, 0} J, \\
& e_{n} \longmapsto-\sum_{m, k \in \mathbb{Z}} x_{k+m+n} \partial_{x_{k}} \partial_{x_{m}}+\sum_{k>0} y_{k} \partial_{x_{-k-n}}+2 K \sum_{m>0} m \partial_{y_{m}} \partial_{x_{m-n}}+(K n+J) \partial_{x_{-n}},
\end{aligned}
$$

em que $c$ age como multiplicação por um escalar $K$ e $h_{0}$ age como multiplicação por um escalar $J$. Conforme os critérios enunciados no Capítulo 2, trata-se de um módulo irredutível se e somente se $K \neq 0$. Se $K=0$ e $J \neq 0$, o quociente deste módulo pelo submódulo gerado pelos elementos da forma $y_{m}, m>0$ é irredutível.

Observamos ainda que, tratando-se do módulo de Verma imaginário $\tilde{M}(\lambda), \lambda \in \mathfrak{h}^{*}, \lambda(c)=K$, $\lambda\left(h_{0}\right)=J$.

Verificaremos agora que trata-se realmente de um homomorfismo de álgebras de Lie: sejam $n_{1}, n_{2} \in \mathbb{Z}$, então 


$$
\begin{aligned}
& {\left[\rho\left(h_{n_{1}}\right), \rho\left(e_{n_{2}}\right)\right]=\left[-2 \sum_{m \in \mathbb{Z}} x_{m+n_{1}} \partial_{x_{m}}+\delta_{n_{1}<0} y_{-n_{1}}+\delta_{n_{1}>0} 2 n_{1} K \partial_{y_{n_{1}}}+\delta_{n_{1}, 0} J,\right.} \\
& \left.-\sum_{j, k \in \mathbb{Z}} x_{k+j+n_{2}} \partial_{x_{k}} \partial_{x_{j}}+\sum_{k>0} y_{k} \partial_{x_{-k-n_{2}}}+2 K \sum_{j>0} j \partial_{y_{j}} \partial_{x_{j-n_{2}}}+\left(K n_{2}+J\right) \partial_{x_{-n_{2}}}\right] \\
& =2\left[\sum_{m \in \mathbb{Z}} x_{m+n_{1}} \partial_{x_{m}}, \sum_{j, k \in \mathbb{Z}} x_{k+j+n_{2}} \partial_{x_{k}} \partial_{x_{j}}\right]-2\left[\sum_{m \in \mathbb{Z}} x_{m+n_{1}} \partial_{x_{m}}, \sum_{k>0} y_{k} \partial_{x_{-k-n_{2}}}\right] \\
& -2\left[\sum_{m \in \mathbb{Z}} x_{m+n_{1}} \partial_{x_{m}}, 2 K \sum_{j>0} j \partial_{y_{j}} \partial_{x_{j-n_{2}}}\right]-2\left[\sum_{m \in \mathbb{Z}} x_{m+n_{1}} \partial_{x_{m}},\left(K n_{2}+J\right) \partial_{x_{-n_{2}}}\right] \\
& +\left[\delta_{n_{1}<0} y_{-n_{1}}, 2 K \sum_{j>0} j \partial_{y_{j}} \partial_{x_{j-n_{2}}}\right]+\left[\delta_{n_{1}>0} 2 n_{1} K \partial_{y_{n_{1}}}, \sum_{k>0} y_{k} \partial_{x_{-k-n_{2}}}\right] \\
& =^{(*)} 2 \sum_{j, k \in \mathbb{Z}} x_{k+j+n_{1}+n_{2}} \sum_{j, k \in \mathbb{Z}} \partial_{x_{k}} \partial_{x_{j}}-2 \sum_{j, m \in \mathbb{Z}} x_{m+n_{1}+j+n_{2}} \partial_{x_{j}} \partial_{x_{m}}-2 \sum_{k, m \in \mathbb{Z}} x_{k+m+n_{1}+n_{2}} \partial_{x_{k}} \partial_{x_{m}} \\
& +2 \sum_{k>0} y_{k} \partial_{x_{-k-\left(n_{1}+n_{2}\right)}}+4 K \sum_{j>0} j \partial_{y_{j}} \partial_{x_{j-\left(n_{1}+n_{2}\right)}}+2\left(K n_{2}+J\right) \partial_{x_{-\left(n_{1}+n_{2}\right)}}+2 K n_{1} \partial_{x_{-\left(n_{1}+n_{2}\right)}} \\
& =\rho\left(\left[h_{n_{1}}, e_{n_{2}}\right]\right) \text {; } \\
& {\left[\rho\left(h_{n_{1}}\right), \rho\left(f_{n_{2}}\right)\right]=\left[-2 \sum_{m \in \mathbb{Z}} x_{m+n_{1}} \partial_{x_{m}}+\delta_{n_{1}<0} y_{-n_{1}}+\delta_{n_{1}>0} 2 n_{1} K \partial_{y_{n_{1}}}+\delta_{n_{1}, 0} J, x_{n_{2}}\right]} \\
& =\left[-2 \sum_{m \in \mathbb{Z}} x_{m+n_{1}} \partial_{x_{m}}, x_{n_{2}}\right]=-2 x_{n_{1}+n_{2}} \\
& =\rho\left(\left[h_{n_{1}}, f_{n_{2}}\right]\right) \text {; } \\
& {\left[\rho\left(h_{n_{1}}\right), \rho\left(h_{n_{2}}\right)\right]=\left[-2 \sum_{m \in \mathbb{Z}} x_{m+n_{1}} \partial_{x_{m}}+\delta_{n_{1}<0} y_{-n_{1}}+\delta_{n_{1}>0} 2 n_{1} K \partial_{y_{n_{1}}}+\delta_{n_{1}, 0} J\right.} \\
& \left.-2 \sum_{j \in \mathbb{Z}} x_{j+n_{2}} \partial_{x_{j}}+\delta_{n_{2}<0} y_{-n_{2}}+\delta_{n_{2}>0} 2 n_{2} K \partial_{y_{n_{2}}}+\delta_{n_{2}, 0} J\right] \\
& =4\left[\sum_{m \in \mathbb{Z}} x_{m+n_{1}} \partial_{x_{m}}, \sum_{j \in \mathbb{Z}} x_{j+n_{2}} \partial_{x_{j}}\right]+\left[\delta_{n_{1}<0} y_{-n_{1}}, \delta_{n_{2}>0} 2 n_{2} K \partial_{y_{n_{2}}}\right] \\
& +\left[\delta_{n_{1}>0} 2 n_{1} K \partial_{y_{n_{1}}}, \delta_{n_{2}<0} y_{-n_{2}}\right] \\
& { }^{(*)} 4 \sum_{j \in \mathbb{Z}} x_{j+n_{1}+n_{2}} \sum_{j \in \mathbb{Z}} \partial_{x_{j}}-4 \sum_{m \in \mathbb{Z}} x_{m+n_{1}+n_{2}} \sum_{m \in \mathbb{Z}} \partial_{x_{m}}+2 \delta_{n_{1},-n_{2}} K n_{1} \\
& =4 \sum_{j \in \mathbb{Z}} x_{j+n_{1}+n_{2}} \partial_{x_{j}}-4 \sum_{m \in \mathbb{Z}} x_{m+n_{1}+n_{2}} \partial_{x_{m}}+2 \delta_{n_{1},-n_{2}} K n_{1} \\
& =2 \delta_{n_{1},-n_{2}} K n_{1}=\rho\left(\left[h_{n_{1}}, h_{n_{2}}\right]\right) \text {; }
\end{aligned}
$$




$$
\begin{aligned}
{\left[\rho\left(e_{n_{1}}\right), \rho\left(f_{n_{2}}\right)\right] } & =\left[-\sum_{m, k \in \mathbb{Z}} x_{k+m+n_{1}} \partial_{x_{k}} \partial_{x_{m}}+\sum_{k>0} y_{k} \partial_{x_{-k-n_{1}}}+2 K \sum_{m>0} m \partial_{y_{m}} \partial_{x_{m-n_{1}}}+\left(K n_{1}+J\right) \partial_{x_{-n_{1}}}, x_{n_{2}}\right] \\
& =-\left[\sum_{m, k \in \mathbb{Z}} x_{k+m+n_{1}} \partial_{x_{k}} \partial_{x_{m}}, x_{n_{2}}\right]+\left[\sum_{k>0} y_{k} \partial_{x_{-k-n_{1}}}, x_{n_{2}}\right] \\
& +2 K\left[\sum_{m>0} m \partial_{y_{m}} \partial_{x_{m-n_{1}}}, x_{n_{2}}\right]+\left[\left(K n_{1}+J\right) \partial_{x_{-n_{1}}}, x_{n_{2}}\right] \\
& =-\sum_{k \in \mathbb{Z}} x_{k+n_{1}+n_{2}} \partial_{x_{k}}-\sum_{m \in \mathbb{Z}} x_{m+n_{1}+n_{2}} \partial_{x_{m}}+\delta_{\left(n_{1}+n_{2}\right)<0} y_{-\left(n_{1}+n_{2}\right)} \\
& +2 K \delta_{n_{1}+n_{2}>0}\left(n_{1}+n_{2}\right) \partial_{y_{n_{1}+n_{2}}}+\left(K n_{1}+J\right) \delta_{n_{1}+n_{2}, 0} \\
& =-2 \sum_{j \in \mathbb{Z}} x_{j+n_{1}+n_{2}} \partial_{x_{j}}+\delta_{n_{1}+n_{2}<0} y_{-\left(n_{1}+n_{2}\right)}+\delta_{n_{1}+n_{2}>0} 2\left(n_{1}+n_{2}\right) K \partial_{y_{n_{1}+n_{2}}} \\
& +\delta_{n_{1}+n_{2}, 0} J+n_{1} K \delta_{n_{1}+n_{2}, 0}=\rho\left(\left[e_{n_{1}}, f_{n_{2}}\right]\right) .
\end{aligned}
$$

$(*)$ :ou $\delta_{n_{1}>0}=0$ ou $\delta_{n_{1}<0}=0$.

\subsubsection{Segunda Realização de Campos Livres}

Existe um outro caminho através do qual as fórmulas obtidas no Exemplo 3.2.1 podem ser corrigidas, o que leva à segunda realização de campos livres. Embora não a estejamos utilizando neste trabalho, faremos algumas considerações a respeito.

Para tanto, denotemos $a_{n}=\partial_{x_{n}}, a_{n}^{*}=x_{-n}, n \in \mathbb{Z}$, e consideremos as séries formais de potências:

$$
a(z)=\sum_{n \in \mathbb{Z}} a_{n} z^{-n-1}, a^{*}(z)=\sum_{n \in \mathbb{Z}} a_{n}^{*} z^{-n}
$$

As séries (3.3) são chamadas de distribuições formais.

Consideremos ainda uma distribuição formal especial, chamada de função formal delta e denotada por $\delta(z, w)=\sum_{n \in \mathbb{Z}} z^{-n-1} w^{n}$. Ao longo desta seção também surgirá a distribuição formal $\delta(w, z)=\sum_{n \in \mathbb{Z}} w^{-n-1} z^{n}$. Usaremos ambas, sem distinção, de acordo com a necessidade, pois conforme [13], $\delta(z, w)=\delta(w, z)$.

Notemos que $\left[a_{n}, a_{m}^{*}\right]=\delta_{n+m, 0}$ e $\left[a_{n}, a_{m}\right]=\left[a_{n}^{*}, a_{m}^{*}\right]=0$, para todo $n, m \in \mathbb{Z}$. Utilizando esses conceitos, as fórmulas no Exemplo 3.2.1 podem ser reescritas como segue:

$$
e(z) \longmapsto a(z), h(z) \longmapsto-2 a^{*}(z) a(z), f(z) \longmapsto-a^{*}(z)^{2} a(z), c \longmapsto 0,
$$

em que $g(z)=\sum_{n \in \mathbb{Z}} g_{n} z^{-n-1}$, para $g \in\{e, f, h\}$.

Nosso objetivo é obter, a partir dessas fórmulas, utilizando a linguagem de distribuições formais, uma representação $\rho: \hat{\mathfrak{s l}}(2, \mathbb{C}) \longrightarrow \mathfrak{g l}\left(\mathbb{C}\left[x_{m}, m \in \mathbb{Z}\right]\right)$.

Para verificar se o colchete de Lie é preservado, faremos uso do seguinte teorema: 
Teorema 3.2.2. Em $\hat{\mathfrak{s l}}(2, \mathbb{C})$ são válidas as seguintes relações:

(a) $[h(z), e(w)]=2 \delta(z, w) e(w)$;

(b) $[h(z), f(w)]=-2 \delta(z, w) f(w)$;

(c) $[e(z), f(w)]=\delta(z, w) h(w)+\partial_{w} \delta(z, w) c$;

(d) $[h(z), h(w)]=2 c \partial_{w} \delta(z, w)$,

em que $\partial_{z}$ e $\partial_{w}$ são as derivadas de $\delta(z, w)$ nas variáveis $z$ e $w$ respectivamente.

Demonstração.

$$
\begin{aligned}
& {[h(z), e(w)]=\left[\sum_{n \in \mathbb{Z}} h_{n} z^{-n-1}, \sum_{m \in \mathbb{Z}} e_{m} w^{-m-1}\right]=\sum_{n, m \in \mathbb{Z}}\left[h_{n}, e_{m}\right] z^{-n-1} w^{-m-1}} \\
& =\sum_{n, m \in \mathbb{Z}}[h, e]_{n+m} z^{-n-1} w^{-m-1}+\sum_{n, m \in \mathbb{Z}} n \delta_{n,-m} \underbrace{(h \mid e)}_{0} c z^{-n-1} w^{-m-1} \\
& { }^{(*)} \sum_{n \in \mathbb{Z}} z^{-n-1} w^{n} \sum_{m \in \mathbb{Z}}[h, e]_{m} w^{-m-1} \\
& =2 \delta(z, w) e(w) \\
& {[h(z), f(w)]=\left[\sum_{n \in \mathbb{Z}} h_{n} z^{-n-1}, \sum_{m \in \mathbb{Z}} f_{m} w^{-m-1}\right]=\sum_{n, m \in \mathbb{Z}}\left[h_{n}, f_{m}\right] z^{-n-1} w^{-m-1}} \\
& =\sum_{n, m \in \mathbb{Z}}[h, f]_{n+m} z^{-n-1} w^{-m-1}+\sum_{n, m \in \mathbb{Z}} n \delta_{n,-m} \underbrace{(h \mid f)}_{0} c z^{-n-1} w^{-m-1} \\
& =^{(*)} \sum_{n \in \mathbb{Z}} z^{-n-1} w^{n} \sum_{m \in \mathbb{Z}}[h, f]_{m} w^{-m-1} \\
& =-2 \delta(z, w) f(w) \\
& {[e(z), f(w)]=\left[\sum_{n \in \mathbb{Z}} e_{n} z^{-n-1}, \sum_{m \in \mathbb{Z}} f_{m} w^{-m-1}\right]=\sum_{n, m \in \mathbb{Z}}\left[e_{n}, f_{m}\right] z^{-n-1} w^{-m-1}} \\
& =\sum_{n, m \in \mathbb{Z}}[e, f]_{n+m} z^{-n-1} w^{-m-1}+\sum_{n, m \in \mathbb{Z}} n \delta_{n,-m} \underbrace{(e \mid f)}_{1} c z^{-n-1} w^{-m-1} \\
& { }^{(*)} \sum_{n \in \mathbb{Z}} z^{-n-1} w^{n} \sum_{m \in \mathbb{Z}}[e, f]_{m} w^{-m-1}-\sum_{m \in \mathbb{Z}} m c z^{m-1} w^{-m-1} \\
& =\delta(z, w) h(w)-\partial_{z} \delta(w, z) c=\delta(z, w) h(w)-\partial_{z} \delta(z, w) c \\
& =\delta(z, w) h(w)+\partial_{w} \delta(z, w) c ; \\
& {[h(z), h(w)]=\left[\sum_{n \in \mathbb{Z}} h_{n} z^{-n-1}, \sum_{m \in \mathbb{Z}} h_{m} w^{-m-1}\right]=\sum_{n, m \in \mathbb{Z}}\left[h_{n}, h_{m}\right] z^{-n-1} w^{-m-1}} \\
& =\sum_{n, m \in \mathbb{Z}} \underbrace{[h, h]_{n+m}}_{0} z^{-n-1} w^{-m-1}+\sum_{n, m \in \mathbb{Z}} n \delta_{n,-m} \underbrace{(h \mid h)}_{2} c z^{-n-1} w^{-m-1} \\
& =-2 \sum_{m \in \mathbb{Z}} m c z^{m-1} w^{-m-1} \\
& =-2 c \partial_{z} \delta(w, z)=-2 c \partial_{z} \delta(z, w)=2 c \partial_{w} \delta(z, w) .
\end{aligned}
$$


(*):como $n, m \in \mathbb{Z}$, para cada $n$ fixado, trocamos $m$ por $m-n$.

Destacamos que fizemos uso da igualdade $\partial_{w} \delta(z, w)=-\partial_{z} \delta(z, w)$, conforme [13].

Verificaremos agora que o colchete realmente é preservado. Utilizaremos repetidas vezes a seguinte igualdade:

$$
\begin{aligned}
{\left[a(w), a^{*}(z)\right] } & =\left[\sum_{m \in \mathbb{Z}} a_{m} w^{-m-1}, \sum_{n \in \mathbb{Z}} a_{n}^{*} z^{-n}\right]=\left(\sum_{m, n \in \mathbb{Z}}\left[a_{m}, a_{n}^{*}\right] w^{-m-1} z^{-n}\right) \\
& =\left(\sum_{m \in \mathbb{Z}} w^{-m-1} z^{m}\right)=\delta(w, z)=\delta(z, w) .
\end{aligned}
$$

Utilizaremos também as igualdades $\delta(z, w) a(w)=\delta(z, w) a(z)$ e $\partial_{w} \delta(z, w)=-\partial_{z} \delta(z, w)$, conforme [13]. 


$$
\begin{aligned}
& {\left[-2 a^{*}(z) a(z), a(w)\right]=-2 a^{*}(z) \underbrace{[a(z), a(w)]}_{0}-2\left[a^{*}(z), a(w)\right] a(z)} \\
& =2\left[a(w), a^{*}(z)\right] a(z)=^{3.4} 2 \delta(w, z) a(z)=2 \delta(z, w) a(w) ; \\
& {\left[-2 a^{*}(z) a(z),-a^{*}(w) a^{*}(w) a(w)\right]=2\left[a^{*}(z) a(z), a^{*}(w) a^{*}(w) a(w)\right]} \\
& =2\left[a^{*}(z) a(z), a^{*}(w)\right] a^{*}(w) a(w) \\
& +2 a^{*}(w)\left[a^{*}(z) a(z), a^{*}(w)\right] a(w)+2 a^{*}(w) a^{*}(w)\left[a^{*}(z) a(z), a(w)\right] \\
& =2 a^{*}(z)\left[a(z), a^{*}(w)\right] a^{*}(w) a(w)+2 \underbrace{\left[a^{*}(z), a^{*}(w)\right]}_{0} a(z) a^{*}(w) a(w) \\
& +2 a^{*}(w) a^{*}(z)\left[a(z), a^{*}(w)\right] a(w)+2 a^{*}(w) \underbrace{\left[a^{*}(z), a^{*}(w)\right]}_{0} a(z) a(w) \\
& +2 a^{*}(w) a^{*}(w) a^{*}(z) \underbrace{[a(z), a(w)]}_{0}-2 a^{*}(w) a^{*}(w)\left[a(w), a^{*}(z)\right] a(z) \\
& ={ }^{3.4} 2 a^{*}(z) \delta(z, w) a^{*}(w) a(w) \\
& +2 a^{*}(w) a^{*}(z) \delta(z, w) a(w)-2 a^{*}(w) a^{*}(w) \delta(w, z) a(z) \\
& =2 \delta(z, w) a^{*}(w)^{2} a(w) ; \\
& {\left[a(z),-a^{*}(w) a^{*}(w) a(w)\right]=-\left[a(z), a^{*}(w) a^{*}(w) a(w)\right]} \\
& =-\left[a(z), a^{*}(w)\right] a^{*}(w) a(w)-a^{*}(w)\left[a(z), a^{*}(w)\right] a(w)-a^{*}(w) a^{*}(w) \underbrace{[a(z), a(w)]}_{0} \\
& ={ }^{3.4}-\delta(z, w) a^{*}(w) a(w)-a^{*}(w) \delta(z, w) a(w) \\
& =-2 \delta(z, w) a^{*}(w) a(w) ; \\
& {\left[-2 a^{*}(z) a(z),-2 a^{*}(w) a(w)\right]=4\left[a^{*}(z) a(z), a^{*}(w) a(w)\right]} \\
& =4\left[a^{*}(z) a(z), a^{*}(w)\right] a(w)+4 a^{*}(w)\left[a^{*}(z) a(z), a(w)\right] \\
& =4 a^{*}(z)\left[a(z), a^{*}(w)\right] a(w)+4 \underbrace{\left[a^{*}(z), a^{*}(w)\right]}_{0} a(z) a(w) \\
& +4 a^{*}(w) a^{*}(z) \underbrace{[a(z), a(w)]}_{0}+4 a^{*}(w)\left[a^{*}(z), a(w)\right] a(z) \\
& =4 a^{*}(z)\left[a(z), a^{*}(w)\right] a(w)-4 a^{*}(w)\left[a(w), a^{*}(z)\right] a(z) \\
& ={ }^{3.4}-4 a^{*}(w) \delta(w, z) a(z)+4 a^{*}(z) \delta(z, w) a(w) \\
& =-4 \delta(z, w) a^{*}(w) a(w)+4 \delta(z, w) a^{*}(w) a(w)=0 .
\end{aligned}
$$

Essa realização não está bem definida, pois os operadores aniquilação e criação não estão ainda na ordem correta. Para torná-la bem definida, aplicamos as duas anti-involuções (3.2), e temos as fórmulas:

$$
f(z) \longmapsto a(z), h(z) \longmapsto 2 a(z) a^{*}(z), e(z) \longmapsto-a(z) a^{*}(z)^{2},
$$

em que $a_{n}$ e $a_{n}^{*}$ têm agora o seguinte significado $a_{n}=x_{n}$ e $a_{n}^{*}=-\partial_{x_{-n}}$. Esse é um quociente do módulo de Verma imaginário.

Uma abordagem diferente, que introduz o conceito de ordem normal, foi sugerida em [21]. Denote 


$$
a(z)_{-}=\sum_{n<0} a_{n} z^{-n-1}, a(z)_{+}=\sum_{n \geq 0} a_{n} z^{-n-1} \text { e } b(z)=\sum_{n \in \mathbb{Z}} b_{n} z^{-n-1}
$$

e defina a ordem normal como segue:

$$
: a(z) b(z):=a(z) \_b(z)+b(z) a(z)_{+},
$$

em que

$$
a_{n}=\left\{\begin{array}{cc}
x_{n}, & n<0 \\
\partial_{x_{n}}, & n \geq 0
\end{array}, a_{n}^{*}=\left\{\begin{array}{cc}
x_{-n}, & n \leq 0 \\
-\partial_{x_{-n}}, & n>0
\end{array}, b_{m}=\left\{\begin{array}{cc}
2 K m \partial_{y_{m}} & m \geq 0 \\
y_{-m}, & m<0
\end{array} .\right.\right.\right.
$$

Notemos que $\left[a_{n}, a_{m}^{*}\right]=\delta_{n+m, 0}$ e $\left[b_{n}, b_{m}\right]=2 K n \delta_{n+m, 0}$, para todo $m, n \in \mathbb{Z}$.

Com essa notação, temos o seguinte teorema:

Teorema 3.2.3. [21] As fórmulas

$$
\begin{array}{r}
c \longmapsto K, e(z) \longmapsto a(z), h(z) \longmapsto-2: a^{*}(z) a(z):+b(z), \\
f(z) \longmapsto-: a^{*}(z)^{2} a(z):+K \partial_{z} a^{*}(z)+a^{*}(z) b(z)
\end{array}
$$

definem a segunda realização de campos livres $\rho: \hat{\mathfrak{s}}(2, \mathbb{C}) \longrightarrow \mathfrak{g l}\left(\mathbb{C}\left[x_{n}, n \in \mathbb{Z}\right] \otimes \mathbb{C}\left[y_{m}, m>0\right]\right)$ da álgebra de Lie afim $\hat{\mathfrak{s} l}(2, \mathbb{C})$.

Mostraremos que as relações do Teorema 3.2.2 estão satisfeitas. Novamente, utilizaremos a relação (3.4) e as igualdades $\partial_{w} \delta(z, w)=-\partial_{z} \delta(z, w)$ e $\delta(z, w) a(z)=\delta(z, w) a(w)$. A igualdade $: a(z) b(z) c(z):=: a(z)(: b(z) c(z):):([5])$ também será necessária. 
Demonstração.

$$
\begin{aligned}
{[\rho(h)(z), \rho(e)(w)] } & =\left[-2: a^{*}(z) a(z):+b(z), a(w)\right]=\left[-2\left(a^{*}(z)-a(z)+a(z) a^{*}(z)_{+}\right)+b(z), a(w)\right] \\
& =-2\left[a^{*}(z)_{-} a(z), a(w)\right]-2\left[a(z) a^{*}(z)_{+}, a(w)\right]+\underbrace{[b(z), a(w)]}_{0} \\
& =-2 a^{*}(z)_{-} \underbrace{[a(z), a(w)]}_{0}+2\left[a(w), a^{*}(z)_{-}\right] a(z)-2 a(z)\left[a^{*}(z)_{+}, a(w)\right] \\
& +2 \underbrace{[a(w), a(z)]}_{0} a^{*}(z)_{+} \\
& =2\left[\sum_{n \in \mathbb{Z}} a_{n} w^{-n-1}, \sum_{m<0} a_{m}^{*} z^{-m}\right] a(z)+2 a(z)\left[\sum_{n \in \mathbb{Z}} a_{n} w^{-n-1}, \sum_{m \geq 0} a_{m}^{*} z^{-m}\right] \\
& =2\left(\sum_{n>0} w^{-n-1} z^{n}\right) a(z)+2 a(z)\left(\sum_{n \leq 0} w^{-n-1} z^{n}\right) \\
& =2 a(z)\left(\sum_{n>0} w^{-n-1} z^{n}+\sum_{n \leq 0} w^{-n-1} z^{n}\right) \\
& =2 \delta(w, z) a(z)=2 \delta(z, w) a(w) ;
\end{aligned}
$$




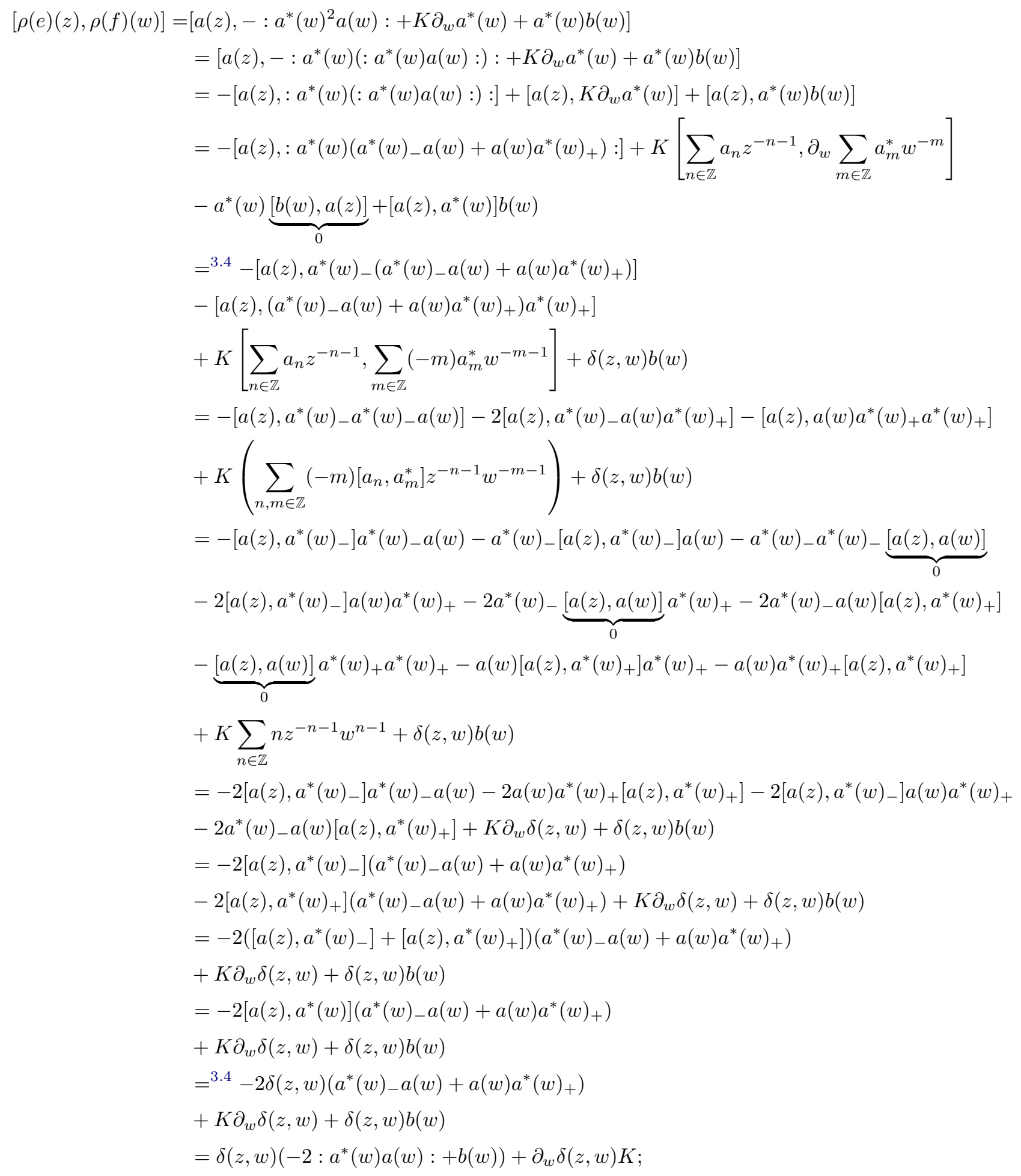




$$
\begin{aligned}
& {[\rho(h)(z), \rho(h)(w)]=\left[-2: a^{*}(z) a(z):+b(z),-2: a^{*}(w) a(w):+b(w)\right]} \\
& =4\left[: a^{*}(z) a(z):,: a^{*}(w) a(w):\right]-2\left[: a^{*}(z) a(z):, b(w)\right]-2\left[b(z),: a^{*}(w) a(w):\right]+[b(z), b(w)] \\
& =4\left[a^{*}(z)_{-} a(z)+a(z) a^{*}(z)_{+}, a^{*}(w)_{-} a(w)+a(w) a^{*}(w)_{+}\right]-2 \underbrace{\left[: a^{*}(z) a(z):, b(w)\right]}_{0} \\
& -2 \underbrace{\left[b(z),: a^{*}(w) a(w):\right]}_{0}+\left[\sum_{n \in \mathbb{Z}} b_{n} z^{-n-1}, \sum_{m \in \mathbb{Z}} b_{m}^{-m-1}\right] \\
& =4\left[a^{*}(z)_{-} a(z), a^{*}(w)_{-} a(w)\right]+4\left[a^{*}(z)_{-} a(z), a(w) a^{*}(w)_{+}\right] \\
& +4\left[a(z) a^{*}(z)_{+}, a^{*}(w)_{-} a(w)\right]+4\left[a(z) a^{*}(z)_{+}, a(w) a^{*}(w)_{+}\right]+\sum_{n, m \in \mathbb{Z}}\left[b_{n}, b_{m}\right] z^{-n-1} w^{-m-1} \\
& =4\left[a^{*}(z)_{-} a(z), a^{*}(w)_{-}\right] a(w)+4 a^{*}(w)_{-}\left[a^{*}(z)_{-} a(z), a(w)\right] \\
& +4\left[a^{*}(z)_{-} a(z), a(w)\right] a^{*}(w)_{+}+4 a(w)\left[a^{*}(z)_{-} a(z), a^{*}(w)_{+}\right] \\
& +4\left[a(z) a^{*}(z)_{+}, a^{*}(w)_{-}\right] a(w)+4 a^{*}(w)_{-}\left[a(z) a^{*}(z)_{+}, a(w)\right] \\
& +4\left[a(z) a^{*}(z)_{+}, a(w)\right] a^{*}(w)_{+}+4 a(w)\left[a(z) a^{*}(z)_{+}, a^{*}(w)_{+}\right]+\sum_{n, m \in \mathbb{Z}} 2 n K \delta_{n+m, 0} z^{-n-1} w^{-m-1} \\
& =4 a^{*}(z)_{-}\left[a(z), a^{*}(w)_{-}\right] a(w)+4 \underbrace{\left[a^{*}(z)_{-}, a^{*}(w)_{-}\right]}_{0} a(z) a(w) \\
& +4 a^{*}(w)_{-} a^{*}(z)_{-} \underbrace{[a(z), a(w)]}_{0}+4 a^{*}(w)_{-}\left[a^{*}(z)_{-}, a(w)\right] a(z) \\
& +4 a^{*}(z)_{-} \underbrace{[a(z), a(w)]}_{0} a^{*}(w)_{+}+4\left[a^{*}(z)_{-}, a(w)\right] a(z) a^{*}(w)_{+} \\
& +4 a(w) a^{*}(z)_{-}\left[a(z), a^{*}(w)_{+}\right]+4 a(w) \underbrace{\left[a^{*}(z)_{-}, a^{*}(w)_{+}\right]}_{0} a(z) \\
& +4 a(z) \underbrace{\left[a^{*}(z)_{+}, a^{*}(w)_{-}\right.}_{0}] a(w)+4\left[a(z), a^{*}(w)_{-}\right] a^{*}(z)_{+} a(w) \\
& +4 a^{*}(w)_{-} a(z)\left[a^{*}(z)_{+}, a(w)\right]+4 a^{*}(w)_{-} \underbrace{[a(z), a(w)}_{0} a^{*}(z)_{+} \\
& +4 a(z)\left[a^{*}(z)_{+}, a(w)\right] a^{*}(w)_{+}+4 \underbrace{[a(z), a(w)]}_{0} a^{*}(z)_{+} a^{*}(w)_{+} \\
& +4 a(w) a(z) \underbrace{\left[a^{*}(z)_{+}, a^{*}(w)_{+}\right.}_{0}]+4 a(w)\left[a(z), a^{*}(w)_{+}\right] a^{*}(z)_{+}-2 K \sum_{m \in \mathbb{Z}} m z^{m-1} w^{-m-1} \\
& =4 a^{*}(z)_{-}\left[a(z), a^{*}(w)_{-}\right] a(w)-4 a^{*}(w)_{-}\left[a(w), a^{*}(z)_{-}\right] a(z)-4\left[a(w), a^{*}(z)_{-}\right] a(z) a^{*}(w)_{+}
\end{aligned}
$$

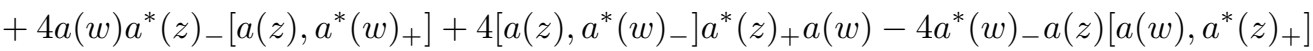

$$
\begin{aligned}
& -4 a(z)\left[a(w), a^{*}(z)_{+}\right] a^{*}(w)_{+}+4 a(w)\left[a(z), a^{*}(w)_{+}\right] a^{*}(z)_{+}-2 K \partial_{z} \delta(w, z) \\
& =-4 a^{*}(w)_{-} a(z)\left(\left[a(w), a^{*}(z)_{-}\right]+\left[a(w), a^{*}(z)_{+}\right]\right) \\
& -4 a(z) a^{*}(w)_{+}\left(\left[a(w), a^{*}(z)_{-}\right]+\left[a(w), a^{*}(z)_{+}\right]\right) \\
& +4 a^{*}(z)_{-} a(w)\left(\left[a(z), a^{*}(w)_{-}\right]+\left[a(z), a^{*}(w)_{+}\right]\right)+4\left[a(w), a^{*}(z)_{-}\right]\left[a(z), a^{*}(w)_{+}\right] \\
& +4 a(w) a^{*}(z)_{+}\left(\left[a(z), a^{*}(w)_{+}\right]+\left[a(z), a^{*}(w)_{-}\right]\right)-4\left[a(z), a^{*}(w)_{-}\right]\left[a(w), a^{*}(z)_{+}\right] \\
& -2 K \partial_{z} \delta(w, z) \\
& ={ }^{3.4,(\star)} 4\left[a(w), a^{*}(z)_{-}\right]\left[a(z), a^{*}(w)_{+}\right]-4\left[a(z), a^{*}(w)_{-}\right]\left[a(w), a^{*}(z)_{+}\right]-2 K \partial_{z} \delta(w, z) \\
& =4\left[a(w), a^{*}(z)_{-}+a^{*}(z)_{+}\right]\left[a(z), a^{*}(w)_{+}\right]-4\left[a(w), a^{*}(z)_{+}\right]\left[a(z), a^{*}(w)_{+}\right] \\
& -4\left[a(z), a^{*}(w)_{-}+a^{*}(w)_{+}\right]\left[a(w), a^{*}(z)_{+}\right]+4\left[a(z), a^{*}(w)_{+}\right]\left[a(w), a^{*}(z)_{+}\right]-2 K \partial_{z} \delta(w, z) \\
& ={ }^{3.4,(\star \star)}-2 K \partial_{z} \delta(w, z)=2 K \partial_{w} \delta(z, w) \text {; }
\end{aligned}
$$


$(\star):-4 a^{*}(w)_{-} a(z) \delta(w, z)-4 a(z) a^{*}(w)_{+} \delta(w, z)+4 a^{*}(z)_{-} a(w) \delta(z, w)+4 a(w) a^{*}(z)_{+} \delta(z, w)=0$

$(\star \star): 4 \delta(w, z)\left[a(z), a^{*}(w)_{+}\right]-4 \delta(z, w)\left[a(w), a^{*}(z)_{+}\right]=0$

$4\left[a(w), a^{*}(z)_{+}\right]\left(\left[a(z), a^{*}(w)_{+}\right]-\left[a(z), a^{*}(w)_{+}\right]\right)=0$

$$
\begin{aligned}
{[\rho(h)(z), \rho(f)(w)] } & =\left[-2: a^{*}(z) a(z):+b(z),-: a^{*}(w) a^{*}(w) a(w):+K \partial_{w} a^{*}(w)+a^{*}(w) b(w)\right] \\
& =2\left[: a^{*}(z) a(z):,: a^{*}(w) a^{*}(w) a(w):\right]-2\left[: a^{*}(z) a(z):, K \partial_{w} a^{*}(w)\right]-2\left[: a^{*}(z) a(z):, a^{*}(w) b(w)\right] \\
& \underbrace{-\left[b(z),: a^{*}(w) a^{*}(w) a(w):\right]}_{0}+\underbrace{\left[b(z), K \partial_{w} a^{*}(w)\right]}_{0}+\left[b(z), a^{*}(w) b(w)\right] .
\end{aligned}
$$

Para mostrar que esse colchete, em particular, é preservado, são necessários cálculos mais longos do que os anteriores. Sendo assim, serão feitos separadamente, como segue: 


$$
\begin{aligned}
{\left[b(z), a^{*}(w) b(w)\right] } & =\underbrace{\left[b(z), a^{*}(w)\right]}_{0} b(w)+a^{*}(w)[b(z), b(w)] \\
& =a^{*}(w)\left[\sum_{n \in \mathbb{Z}} b_{n} z^{-n-1}, \sum_{m \in \mathbb{Z}} b_{m} w^{-m-1}\right]=a^{*}(w)\left(\sum_{n, m \in \mathbb{Z}}\left[b_{n}, b_{m}\right] z^{-n-1} w^{-m-1}\right) \\
& =a^{*}(w)\left(\sum_{n \in \mathbb{Z}} 2 n K z^{-n-1} w^{n-1}\right)=a^{*}(w) 2 K \partial_{w} \delta(z, w) .
\end{aligned}
$$

$-2\left[: a^{*}(z) a(z):, a^{*}(w) b(w)\right]=-2\left[a^{*}(z)_{-} a(z)+a(z) a^{*}(z)_{+}, a^{*}(w) b(w)\right]$

$$
\begin{aligned}
& =-2\left[a^{*}(z)_{-} a(z), a^{*}(w) b(w)\right]-2\left[a(z) a^{*}(z)_{+}, a^{*}(w) b(w)\right] \\
& =-2\left[a^{*}(z)_{-} a(z), a^{*}(w)\right] b(w)-2 a^{*}(w)\left[a^{*}(z)_{-} a(z), b(w)\right] \\
& -2\left[a(z) a^{*}(z)_{+}, a^{*}(w)\right] b(w)-2 a^{*}(w)\left[a(z) a^{*}(z)_{+}, b(w)\right] \\
& =-2 a^{*}(z)_{-}\left[a(z), a^{*}(w)\right] b(w)-2 \underbrace{\left[a^{*}(z)_{-}, a^{*}(w)\right]}_{0} a(z) b(w) \\
& -2 a^{*}(w) a^{*}(z)_{-} \underbrace{[a(z), b(w)]}_{0}-2 a^{*}(w) \underbrace{\left[a^{*}(z)_{-}, b(w)\right]}_{0} a(z) \\
& -2 a(z) \underbrace{\left[a^{*}(z)_{+}, a^{*}(w)\right]}_{0} b(w)-2\left[a(z), a^{*}(w)\right] a^{*}(z)_{+} b(w) \\
& =-2 a^{*}(w) a(z) \underbrace{\left[a^{*}(z)_{+}, b(w)\right]}_{0}-2 a^{*}(w) \underbrace{[a(z), b(w)]}_{0} a^{*}(z)_{+} \\
& =-2\left[a(z), a^{*}(w)\right]\left(a^{*}(z) \_b(w)+a^{*}(z)_{+} b(w)\right)={ }^{3.4}-2 \delta(z, w) a^{*}(z) b(w) \\
& =-2 \delta(z, w) a^{*}(w) b(w) .
\end{aligned}
$$

$-2\left[: a^{*}(z) a(z):, K \partial_{w} a^{*}(w)\right]=-2\left[a^{*}(z)_{-} a(z)+a(z) a^{*}(z)_{+}, K \partial_{w} a^{*}(w)\right]$

$$
=-2\left[a^{*}(z)_{-} a(z), K \partial_{w} a^{*}(w)\right]-2\left[a(z) a^{*}(z)_{+}, K \partial_{w} a^{*}(w)\right]
$$$$
=-2 K a^{*}(z)_{-}\left[a(z), \partial_{w} a^{*}(w)\right]-2 K \underbrace{\left[a^{*}(z)_{-}, \partial_{w} a^{*}(w)\right]}_{0} a(z)
$$$$
-2 K a(z) \underbrace{\left[a^{*}(z)_{+}, \partial_{w} a^{*}(w)\right]}_{0}-2 K\left[a(z), \partial_{w} a^{*}(w)\right] a^{*}(z)_{+}
$$$$
=-2 K\left[a(z), \partial_{w} a^{*}(w)\right]\left(a^{*}(z)_{-}+a^{*}(z)_{+}\right)=-2 K\left[a(z), \partial_{w} a^{*}(w)\right] a^{*}(z)
$$$$
=-2 K\left[\sum_{n \in \mathbb{Z}} a_{n} z^{-n-1}, \sum_{m \in \mathbb{Z}}(-m) a_{m}^{*} w^{-m-1}\right] a^{*}(z)
$$$$
=-2 K\left(\sum_{n, m \in \mathbb{Z}}(-m)\left[a_{n}, a_{m}^{*}\right] z^{-n-1} w^{-m-1}\right) a^{*}(z)=-2 K\left(\sum_{n \in \mathbb{Z}} n z^{-n-1} w^{n-1}\right) a^{*}(z)
$$$$
=-2 K \partial_{w} \delta(z, w) a^{*}(z) \text {. }
$$

\section{Notemos que}




$$
\begin{aligned}
-2 K \partial_{w} \delta(z, w) a^{*}(z)+2 K \partial_{w} \delta(z, w) a^{*}(w) & =2 K \partial_{z} \delta(z, w) a^{*}(z)+2 K \partial_{w} \delta(z, w) a^{*}(w) \\
& =2 K \sum_{n \in \mathbb{Z}}(-n-1) z^{-n-1-1} w^{n} \sum_{m \in \mathbb{Z}} a_{m}^{*} z^{-m} \\
& +2 K \sum_{n \in \mathbb{Z}} n z^{-n-1} w^{n-1} \sum_{m \in \mathbb{Z}} a_{m}^{*} w^{-m} \\
& ={ }^{(\star)} 2 K \sum_{n, m \in \mathbb{Z}}(-n+m+1-1) a_{m}^{*} z^{-n-1} w^{n} w^{-m-1} \\
& +2 K \sum_{n \in \mathbb{Z}} n z^{-n-1} w^{n} \sum_{m \in \mathbb{Z}} a_{m}^{*} w^{-m-1} \\
& =2 K \sum_{n \in \mathbb{Z}} z^{-n-1} w^{n} \sum_{m \in \mathbb{Z}} m a_{m}^{*} w^{-m-1} \\
& =-2 K \delta(z, w) \partial_{w} a^{*}(w) .
\end{aligned}
$$

$(\star)$ :como $m, n \in \mathbb{Z}$, para cada $m$, podemos trocar $n$ por $n-m-1$. 
$2\left[: a^{*}(z) a(z):,: a^{*}(w) a^{*}(w) a(w):\right]=2\left[a^{*}(z)_{-} a(z),: a^{*}(w) a^{*}(w) a(w):\right]+2\left[a(z) a^{*}(z)_{+},: a^{*}(w) a^{*}(w) a(w):\right]$ $=2\left[a^{*}(z)_{-} a(z), a^{*}(w)_{-} a^{*}(w)_{-} a(w)\right]+2\left[a^{*}(z)_{-} a(z), a^{*}(w)_{-} a(w) a^{*}(w)_{+}\right]$ $+2\left[a^{*}(z)_{-} a(z), a^{*}(w)_{-} a(w) a^{*}(w)_{+}\right]+2\left[a^{*}(z)_{-} a(z), a(w) a^{*}(w)_{+} a^{*}(w)_{+}\right]$ $+2\left[a(z) a^{*}(z)_{+}, a^{*}(w)_{-} a^{*}(w)_{-} a(w)\right]+2\left[a(z) a^{*}(z)_{+}, a^{*}(w)_{-} a(w) a^{*}(w)_{+}\right]$ $+2\left[a(z) a^{*}(z)_{+}, a^{*}(w)_{-} a(w) a^{*}(w)_{+}\right]+2\left[a(z) a^{*}(z)_{+}, a(w) a^{*}(w)_{+} a^{*}(w)_{+}\right]$ $=2\left[a^{*}(z)_{-} a(z), a^{*}(w)_{-}\right] a^{*}(w)_{-} a(w)+2 a^{*}(w)_{-}\left[a^{*}(z)_{-} a(z), a^{*}(w)_{-}\right] a(w)$ $+2 a^{*}(w)_{-} a^{*}(w)_{-}\left[a^{*}(z)_{-} a(z), a(w)\right]+2\left[a^{*}(z)_{-} a(z), a^{*}(w)_{-}\right] a(w) a^{*}(w)_{+}$ $+2 a^{*}(w)_{-}\left[a^{*}(z)_{-} a(z), a(w)\right] a^{*}(w)_{+}+2 a^{*}(w)_{-} a(w)\left[a^{*}(z)_{-} a(z), a^{*}(w)_{+}\right]$ $=2\left[a^{*}(z)_{-} a(z), a^{*}(w)_{-}\right] a(w) a^{*}(w)_{+}+2 a^{*}(w)_{-}\left[a^{*}(z)_{-} a(z), a(w)\right] a^{*}(w)_{+}$ $+2 a^{*}(w)_{-} a(w)\left[a^{*}(z)_{-} a(z), a^{*}(w)_{+}\right]+2\left[a^{*}(z)_{-} a(z), a(w)\right] a^{*}(w)_{+} a^{*}(w)_{+}$ $+2 a(w)\left[a^{*}(z)_{-} a(z), a^{*}(w)_{+}\right] a^{*}(w)_{+}+2 a(w) a^{*}(w)_{+}\left[a^{*}(z)_{-} a(z), a^{*}(w)_{+}\right]$ $+2\left[a(z) a^{*}(z)_{+}, a^{*}(w)_{-}\right] a^{*}(w)_{-} a(w)+2 a^{*}(w)_{-}\left[a(z) a^{*}(z)_{+}, a^{*}(w)_{-}\right] a(w)$ $+2 a^{*}(w)_{-} a^{*}(w)_{-}\left[a(z) a^{*}(z)_{+}, a(w)\right]+2\left[a(z) a^{*}(z)_{+}, a^{*}(w)_{-}\right] a(w) a^{*}(w)_{+}$ $+2 a^{*}(w)_{-}\left[a(z) a^{*}(z)_{+}, a(w)\right] a^{*}(w)_{+}+2 a^{*}(w)_{-} a^{*}(w)_{-}\left[a(z) a^{*}(z)_{+}, a^{*}(w)_{+}\right]$ $+2\left[a(z) a^{*}(z)_{+}, a^{*}(w)_{-}\right] a(w) a^{*}(w)_{+}+2 a^{*}(w)_{-}\left[a(z) a^{*}(z)_{+}, a(w)\right] a^{*}(w)_{+}$ $+2 a^{*}(w)_{-} a(w)\left[a(z) a^{*}(z)_{+}, a^{*}(w)_{+}\right]+2\left[a(z) a^{*}(z)_{+}, a(w)\right] a^{*}(w)_{+} a^{*}(w)_{+}$ $+2 a(w)\left[a(z) a^{*}(z)_{+}, a^{*}(w)_{+}\right] a^{*}(w)_{+}+2 a(w) a^{*}(w)_{+}\left[a(z) a^{*}(z)_{+}, a^{*}(w)_{+}\right]$ $=2 a^{*}(z)_{-}\left[a(z), a^{*}(w)_{-}\right] a^{*}(w)_{-} a(w)+2 a^{*}(w)_{-} a^{*}(z)_{-}\left[a(z), a^{*}(w)_{-}\right] a(w)$ $+2 a^{*}(w)_{-} a^{*}(w)_{-}\left[a^{*}(z)_{-}, a(w)\right] a(z)+2 a^{*}(z)_{-}\left[a(z), a^{*}(w)_{-}\right] a(w) a^{*}(w)_{+}$ $+2 a^{*}(w)_{-}\left[a^{*}(z)_{-}, a(w)\right] a(z) a^{*}(w)_{+}+2 a^{*}(w)_{-} a(w) a^{*}(z)_{-}\left[a(z), a^{*}(w)_{+}\right]$ $+2 a^{*}(z)_{-}\left[a(z), a^{*}(w)_{-}\right] a(w) a^{*}(w)_{+}+2 a^{*}(w)_{-}\left[a^{*}(z)_{-}, a(w)\right] a(z) a^{*}(w)_{+}$ $+2 a^{*}(w)_{-} a(w) a^{*}(z)_{-}\left[a(z), a^{*}(w)_{+}\right]+2\left[a^{*}(z)_{-}, a(w)\right] a(z) a^{*}(w)_{+} a^{*}(w)_{+}$ $+2 a(w) a^{*}(z)_{-}\left[a(z), a^{*}(w)_{+}\right] a^{*}(w)_{+}+2 a(w) a^{*}(w)_{+} a^{*}(z)_{-}\left[a(z), a^{*}(w)_{+}\right]$ $+2\left[a(z), a^{*}(w)_{-}\right] a^{*}(z)_{+} a^{*}(w)_{-} a(w)+2 a^{*}(w)_{-}\left[a(z), a^{*}(w)_{-}\right] a^{*}(z)_{+} a(w)$ $+2 a^{*}(w)_{-} a^{*}(w)_{-} a(z)\left[a^{*}(z)_{+}, a(w)\right]+2\left[a(z), a^{*}(w)_{-}\right] a^{*}(z)_{+} a(w) a^{*}(w)_{+}$ $+2 a^{*}(w)_{-} a(z)\left[a^{*}(z)_{+}, a(w)\right] a^{*}(w)_{+}+2 a^{*}(w)_{-} a(w)\left[a(z), a^{*}(w)_{+}\right] a^{*}(z)_{+}$ $+2\left[a(z), a^{*}(w)_{-}\right] a^{*}(z)_{+} a(w) a^{*}(w)_{+}+2 a^{*}(w)_{-} a(z)\left[a^{*}(z)_{+}, a(w)\right] a^{*}(w)_{+}$ $+2 a^{*}(w)_{-} a(w)\left[a(z), a^{*}(w)_{+}\right] a^{*}(z)_{+}+2 a(z)\left[a^{*}(z)_{+}, a(w)\right] a^{*}(w)_{+} a^{*}(w)_{+}$ $+2 a(w)\left[a(z), a^{*}(w)_{+}\right] a^{*}(z)_{+} a^{*}(w)_{+}+2 a(w) a^{*}(w)_{+}\left[a(z), a^{*}(w)_{+}\right] a^{*}(z)_{+}$ $={ }^{3.4}-2 \delta(w, z) a^{*}(w)_{-} a^{*}(w)_{-} a(z)-4 \delta(w, z) a^{*}(w)_{-} a(z) a^{*}(w)_{+}$

$-2 \delta(w, z) a(z) a^{*}(w)_{+} a^{*}(w)_{+}$

$+4 a^{*}(w)_{-} a^{*}(z)_{-} a(w)\left(\left[a(z), a^{*}(w)_{-}\right]+\left[a(z), a^{*}(w)_{+}\right]\right)$

$+4 a^{*}(w)_{-}\left[a(w), a^{*}(z)_{-}\right]\left[a(z), a^{*}(w)_{+}\right]-4 a^{*}(w)_{+}\left[a(w), a^{*}(z)_{+}\right]\left[a(z), a^{*}(w)_{-}\right]$

$+4 a(w) a^{*}(z)_{+} a^{*}(w)_{+}\left(\left[a(z), a^{*}(w)_{+}\right]+\left[a(z), a^{*}(w)_{-}\right]\right)$

$+4 a^{*}(z)_{-} a(w) a^{*}(w)_{+}\left(\left[a(z), a^{*}(w)_{-}\right]+\left[a(z), a^{*}(w)_{+}\right]\right)$

$+4 a^{*}(w)_{+}\left[a(w), a^{*}(z)_{-}\right]\left[a(z), a^{*}(w)_{+}\right]-4 a^{*}(w)_{-}\left[a(w), a^{*}(z)_{+}\right]\left[a(z), a^{*}(w)_{-}\right]$

$+4 a^{*}(w)_{-} a(w) a^{*}(z)_{+}\left(\left[a(z), a^{*}(w)_{+}\right]+\left[a(z), a^{*}(w)_{-}\right]\right)$

$={ }^{3.4,(\star \star)}-2 \delta(w, z) a^{*}(w)_{-} a^{*}(w)_{-} a(z)-4 \delta(w, z) a^{*}(w)_{-} a(z) a^{*}(w)_{+}$

$-2 \delta(w, z) a(z) a^{*}(w)_{+} a^{*}(w)_{+}$

$+4 \delta(z, w) a^{*}(w)_{-} a^{*}(z)_{-} a(w)+4 \delta(z, w) a(w) a^{*}(z)_{+} a^{*}(w)_{+}$

$+4 a^{*}(z)_{-} a(w) a^{*}(w)_{+} \delta(z, w)+4 a^{*}(w)_{-} a(w) a^{*}(z)_{+} \delta(z, w)$. 
Portanto,

$$
\begin{aligned}
& 2\left[: a^{*}(z) a(z):,: a^{*}(w) a^{*}(w) a(w):\right]=2 \delta(z, w) a^{*}(w)_{-} a^{*}(w)_{-} a(w)+2 \delta(z, w) a(w) a^{*}(w)_{+} a^{*}(w)_{+} \\
& 4 \delta(z, w) a^{*}(w)_{-} a(w) a^{*}(w)_{+}=-2 \delta(z, w)\left(-: a^{*}(w)^{2} a(w):\right) \\
& (\star \star):-4 a^{*}(w)\left[a(w), a^{*}(z)_{+}\right]\left[a(z), a^{*}(w)_{-}\right]+4 a^{*}(w)\left[a(w), a^{*}(z)_{-}\right]\left[a(z), a^{*}(w)_{+}\right]=0
\end{aligned}
$$

Esses módulos são conhecidos como módulos de Wakimoto. 


\section{Capítulo 4}

\section{Localização de Álgebras não Comutativas}

Dados um anel $A$ arbitrário e $S \subseteq A$, nem sempre os elementos de $S$ são inversíveis em $A$. Algumas vezes, porém, precisamos desses inversos com alguma finalidade específica, de onde surge naturalmente o questionamento: existe um outro anel, contendo $A$ como subanel, no qual os elementos de $S$ sejam inversíveis?

Neste capítulo veremos que, sob algumas condições impostas a $S$, é possível construir um anel satisfazendo as condições desejadas. Observamos que estamos particularmente interessados em estudar anéis que não sejam necessariamente comutativos.

Como motivação, dedicaremos uma seção ao caso comutativo. Em seguida, teremos uma seção para o caso não comutativo, que será dividida em duas partes: em primeiro lugar, faremos a construção explícita quando $S$ satisfaz todas as condições para que haja a imersão de $A$ no novo anel; na sequência, retirando uma das condições impostas a $S$, enunciaremos um resultado que mostra que ainda é possível a construção de um novo anel, com algumas propriedades interessantes, mas que não contém o anel original como subanel.

\subsection{Anéis Comutativos}

Seja $A$ um anel comutativo, associativo, com elemento identidade $1 \neq 0$. Dedicaremos esta seção à construção do anel de frações de $A$, como uma generalização da construção do corpo de frações de um domínio de integridade. Em seguida, como uma extensão natural dessa construção, estudaremos também módulos de frações sobre o anel de frações de $A$. Os resultados aqui enunciados encontram-se demonstrados em [20].

A proposição abaixo tem a finalidade de recordar o conceito de corpo de frações.

Proposição 4.1.1. Seja A um dominio de integridade. Então, existem um corpo $\mathbb{K}$ e um homomorfismo injetivo de anéis $f: A \longrightarrow \mathbb{K}$ tais que cada elemento de $\mathbb{K}$ pode ser escrito na forma $f(a) f(s)^{-1}$, para alguns $a, s \in A$, com $s \neq 0$.

Demonstração. Faremos apenas um esboço da demonstração. Seja $S=A \backslash\{0\}$. Estabelecemos uma relação de equivalência $\sim$ em $A \times S$ como segue: para $(a, b),(c, d) \in A \times S$, escrevemos

$$
(a, b) \sim(c, d) \Longleftrightarrow a d=b c
$$


Para cada $(a, b) \in A \times S$, denotaremos a classe de equivalência que contém $(a, b)$ por $a / b$ ou $\frac{a}{b}$.

$\mathrm{O}$ conjunto $\mathbb{K}$ de todas as classes de equivalência de $\sim$ possui uma estrutura de corpo sob as operações de adição e multiplicação abaixo:

$$
\begin{aligned}
\frac{a}{b}+\frac{c}{d} & :=\frac{a d+b c}{b d}, \\
\frac{a c}{b} \frac{c}{d} & :=\frac{a c}{b d},
\end{aligned}
$$

para todo $a / b, c / d \in \mathbb{K}$; o elemento neutro aditivo desse corpo é $0 / 1$, e o elemento identidade é $1 / 1$, (que é igual a $a / a$ para todo $a \in S$ ).

A aplicação $f: A \longrightarrow \mathbb{K}$ definida por $f(a)=a / 1$, para todo $a \in A$, é um homomorfismo injetivo de anéis com as propriedades desejadas.

Devido ao homomorfismo injetivo na Proposição 4.1.1, A pode ser imerso em $\mathbb{K}$ como um subanel, e $\mathbb{K}$ é chamado de corpo de frações do domínio de integridade $A$. Destacamos que qualquer outro corpo de frações de $A$ é isomorfo a $\mathbb{K}$.

Consideremos agora um anel $A$ qualquer, e $S \subseteq A$ um conjunto multiplicativo. Iremos novamente considerar uma relação de equivalência no conjunto $A \times S$. Neste caso, porém, a relação de equivalência a ser definida deverá ser capaz de superar os problemas que podem ocorrer com a eventual presença de divisores de zero em $A$.

Uma vez definida a relação de equivalência conveniente, a construção será muito próxima à que foi efetuada na Proposição 4.1.1, embora na maioria dos casos o resultado final seja um anel, que denotaremos por $S^{-1} A$ e diremos que é o anel de frações de $A$ com respeito a $S$. Esse novo anel pode ter divisores de zero, e embora exista um homomorfismo natural de anéis $f: A \longrightarrow S^{-1} A$, ele não será necessariamente injetivo.

Lema 4.1.2. Sejam $A$ um anel e $S$ um subconjunto multiplicativo de $A$. Definimos a relação $\sim$ em $A \times S$ como segue: para $(a, s),(b, t) \in A \times S$, escrevemos

$$
(a, s) \sim(b, t) \Longleftrightarrow \exists u \in S \operatorname{com} u(t a-s b)=0 .
$$

Então, $\sim$ é uma relação de equivalência em $A \times S$.

(vide $[20, \mathrm{p} .81]$ ).

Para cada $(a, s) \in A \times S$, denotaremos a classe de equivalência que contém $(a, s)$ por $a / s$ ou $\frac{a}{s}$, e o conjunto de todas as classes de equivalência de $\sim$ por $S^{-1} A$.

Proposição 4.1.3. Sejam $A$ um anel e $S$ um subconjunto multiplicativo de $A$. Então, $S^{-1} A$ possui estrutura de anel comutativo, associativo, com elemento identidade, sob as operações de adição e multiplicação abaixo:

$$
\begin{aligned}
\frac{a}{s}+\frac{b}{t} & :=\frac{t a+s b}{s t}, \\
\frac{a}{s} \frac{b}{t} & :=\frac{a b}{s t},
\end{aligned}
$$

para todo $a, b \in A$ e $s, t \in S$. 
Observemos que se $s=t$, então $a / s+b / s=(a+b) / s . S^{-1} A$ é chamado de anel de frações de $A$ com respeito a $S$; seu elemento neutro aditivo é $0 / 1$ e seu elemento identidade é $1 / 1$.

Existe um homomorfismo de anéis $f: A \longrightarrow S^{-1} A$ dado por $f(a)=a / 1$, para todo a $\in A$; $f$ é denominado homomorfismo natural de anéis.

(vide $[20, \mathrm{p} .81-82])$.

Observação 4.1.4. Sejam $a \in A$ e $s \in S$. Então, $a / s=0 / 1$ se e somente se existe $t \in S$ tal que $t(1 a-s 0)=0$, isto é, se e somente se existe $t \in S$ tal que $t a=0$. Assim, o anel $S^{-1} A$ é trivial $(1 / 1=0 / 1)$ se e somente se existe $t \in S$ tal que $t 1=0$, isto é, se e somente se $0 \in S$. Em geral, como não queremos construir anéis triviais, podemos impor a condição adicional $0 \notin S$.

Observação 4.1.5. Sejam $A$ um domínio de integridade e $S=A \backslash\{0\}$ (que é um conjunto multiplicativo). Se construirmos o anel de frações $S^{-1} A$ como no Lema 4.1.2 e Proposição 4.1.3, iremos obter precisamente o corpo de frações de $A$. De fato, como $S$ é o conjunto dos elementos que não são divisores de zero em $A$, para $a, b \in A$ e $s, t \in S$, existe $u \in S \operatorname{com} u(t a-s b)=0$ se e somente se $(t a-s b)=0$. Então, a relação de equivalência usada na Proposição 4.1.3 (para este caso especial) é a mesma usada na Proposição 4.1.1; além disso, as operações de anel no conjunto das classes de equivalência são as mesmas nas duas situações.

Notemos que, neste caso, em que $A$ é um domínio de integridade, o homomorfismo natural de anéis $f: A \longrightarrow S^{-1} A$ é injetivo e $A$ é imerso como um subanel em seu corpo de frações.

Exemplo 4.1.6. Sejam $A$ um anel, $P$ um ideal primo de $A$, e $S=A \backslash P$ : neste caso, $S$ será um subconjunto multiplicativo de $A$ e o anel de frações $S^{-1} A$ será denotado por $A_{P}$.

As propriedades abaixo são importantes para mostrar que, a menos de isomorfismo, o anel de frações de $A$ é único:

Observação 4.1.7. Sejam $A$ um anel, $S \subseteq A$ um conjunto multiplicativo, e $f: A \longrightarrow S^{-1} A$ o homomorfismo natural de anéis. Notemos que $f$ tem as seguintes propriedades:

(a) Para cada $s \in S$, o elemento $f(s)=s / 1$ é uma unidade em $S^{-1} A$, com inverso $1 / s$;

(b) Se $a \in K e r f$, então existe $s \in S$ tal que $s a=0$;

(c) Cada elemento $a / s$ de $S^{-1} A$ (em que $a \in A, s \in S$ ) pode ser escrito como $a / s=f(a) f(s)^{-1}$, uma vez que

$$
\frac{a}{s}=\frac{a}{1} \frac{1}{s}=\frac{a}{1}\left(\frac{s}{1}\right)^{-1}=f(a) f(s)^{-1}
$$

Finalmente, a proposição abaixo nos dá a unicidade:

Proposição 4.1.8. Sejam $A$ um anel $e S \subseteq A$ um conjunto multiplicativo. Suponhamos que $B$ seja um anel comutativo e que exista um homomorfismo de anéis $g: A \longrightarrow B$ satisfazendo as mesmas propriedades satisfeitas por $f$ na Observação 4.1.7. Então, existe um único isomorfismo de anéis $h: S^{-1} A \longrightarrow B$ tal que $h \circ f=g$.

(vide [20, p.87]). 


\subsubsection{Módulos sobre Anéis Comutativos}

Sejam $A$ um anel, e $M$ um $A$-módulo. Esta subseção dedica-se à construção de um módulo de frações para $M$.

Aqui, tanto o elemento neutro aditivo de um anel, como o elemento neutro aditivo de um módulo sobre esse anel, serão denotados apenas por 0.

A próxima proposição será apenas enunciada. Na bibliografia utilizada, a demonstração é deixada como exercício, tendo em vista que os cálculos são análogos aos efetuados no caso do anel de frações de $A$.

Proposição 4.1.9. Sejam $A$ um anel, $S \subseteq A$ um conjunto multiplicativo, e $M$ um A-módulo. Para $(m, s),(n, t) \in M \times S$, definimos a relação $\sim$ como segue:

$$
(m, s) \sim(n, t) \Longleftrightarrow \exists u \in S \operatorname{com} u(t m-s n)=0 .
$$

$A$ relação $\sim$ é uma relação de equivalência em $M \times S$.

Para $(m, s) \in M \times S$, a classe de equivalência de $\sim$ que contém $(m, s)$ será denotada por $m / s$ ou $\frac{m}{s}$, e o conjunto de todas as classes de equivalência de $\sim$ será denotado por $S^{-1} M$. Assim, $S^{-1} M$ tem estrutura de $S^{-1} A$-módulo sob a adição e a lei de composição externa abaixo:

$$
\begin{aligned}
\frac{m}{s}+\frac{n}{t} & :=\frac{t m+s n}{s t}, \\
\frac{a}{s} \frac{n}{t} & :=\frac{a n}{s t},
\end{aligned}
$$

para todo $m, n \in M, s, t \in S$ e a $\in$ A. Se $s=t$, então $m / s+n / s=(m+n) / s . S^{-1} M$ é chamado de módulo de frações de $M$ com respeito a $S$; seu elemento neutro da adição é $0 / 1$, e é igual a $0 / s$, para todo $s \in S$.

Observação 4.1.10. Sejam $A$ um anel, $S \subseteq A$ um conjunto multiplicativo, e $M$ um $A$-módulo. A aplicação $g: M \longrightarrow S^{-1} M$ definida por $g(m)=m / 1$, para todo $m \in M$, é um homomorfismo de $A$-módulos quando $S^{-1} M$ é considerado como um $A$-módulo, usando o homomorfismo natural de anéis $f: A \longrightarrow S^{-1} A$ (isto é, pela restrição de escalares).

A seguir, enunciaremos alguns resultados que ilustram a relação entre os homomorfismos de $A$-módulos e os homomorfismos de $S^{-1} A$-módulos.

Lema 4.1.11. Sejam $A$ um anel, $S \subseteq A$ um conjunto multiplicativo, e $L$ e $M$ módulos sobre $A$. Se $f: L \longrightarrow M$ é um homomorfismo de A-módulos, então $f$ induz um homomorfismo de módulos sobre $S^{-1} A$ :

$$
S^{-1} f: S^{-1} L \longrightarrow S^{-1} M
$$

tal que $S^{-1} f(a / s)=f(a) / s$ para todo $a \in L, s \in S$.

(vide [20, p.170-171]

Observação 4.1.12. Sejam $A$ um anel, $L, M, N$ módulos sobre $A$, e $S$ um subconjunto multiplicativo de $A$. Sejam $f, f^{\prime}: L \longrightarrow M$ e $g: M \longrightarrow N$ homomorfismos de $A$-módulos. Então: 
(a) $S^{-1}\left(f+f^{\prime}\right)=S^{-1} f+S^{-1} f^{\prime}$;

(b) $S^{-1} z$, em que $z$ denota o homomorfismo nulo de $L$ em $M$, é o homomorfismo nulo de $S^{-1} L$ em $S^{-1} M$

(c) $S^{-1}(g \circ f)=S^{-1} g \circ S^{-1} f$;

(d) $S^{-1}\left(I d_{M}\right)=I d_{S^{-1} M}$; e

(e) Se $f$ é um isomorfismo de $A$-módulos, então $S^{-1} f$ é um isomorfismo de $S^{-1} A$-módulos.

Com essa observação, mais precisamente com os itens $(a),(c)$ e $(d)$, temos que " $S^{-1}$ "pode ser visto como um funtor covariante aditivo da categoria dos $A$-módulos e homomorfismos de $A$-módulos na categoria dos $S^{-1} A$-módulos e homomorfismos de $S^{-1} A$-módulos.

Lema 4.1.13. Sejam $A$ um anel, $L, M, N$ módulos sobre $A e$

$$
L \stackrel{f}{\longrightarrow} M \stackrel{g}{\longrightarrow} N
$$

uma sequência exata de módulos e homomorfismos sobre A. Seja $S$ um subconjunto multiplicativo de A. Então

$$
S^{-1} L \stackrel{S^{-1} f}{\longrightarrow} S^{-1} M \stackrel{S^{-1} g}{\longrightarrow} S^{-1} N
$$

é exata também.

(vide [20, p.171-172]).

Proposição 4.1.14. Sejam $A$ um anel e $M$ um A-módulo. Então, os $S^{-1} A$-módulos $S^{-1} M e$ $S^{-1} A \otimes_{A} M$ são isomorfos; mais precisamente, existe um único isomorfismo

$$
f: S^{-1} A \otimes_{A} M \longrightarrow S^{-1} M
$$

para o qual $f((a / s) \otimes m)=a m / s ; \forall a \in A, m \in M, s \in S$.

Notemos que $S^{-1} A \otimes_{A} M$ é um $S^{-1} A$-módulo devido à Definição A.1.6.

(vide [1, p.39-40]).

Observação 4.1.15. Para anéis comutativos e módulos sobre anéis comutativos o processo de construção de anéis e módulos de frações é sempre possível, pois todo anel comutativo possui pelo menos um ideal maximal, que é primo.

\subsection{Anéis não Comutativos}

Seja $A$ um anel associativo, não necessariamente comutativo, com elemento identidade $1 \neq 0$. Nesta seção, como na anterior, vamos considerar um conjunto multiplicativo $S \subseteq A$, e construir o anel de frações de $A$. Mas aqui, diferente do caso comutativo, há duas possibilidades: a construção 
do anel de frações à direita, ou do anel de frações à esquerda, dependendo das condições que sejam satisfeitas por $S$.

Tendo em vista a aplicação prática que os anéis de frações têm neste trabalho, estamos particularmente interessados no caso em que $S$ é um conjunto de Ore formado apenas por elementos regulares de $A$ (0 não é um elemento regular). Estudaremos a construção explícita para este caso, tanto para o anel de frações à esquerda (respectivamente à direita) de $A$, como para módulos de frações sobre ele.

Em seguida, enunciaremos os resultados para o caso geral, em que $S$ é um conjunto multiplicativo que satisfaz apenas uma das condições de Ore (à direita ou à esquerda), e pode possuir elementos não regulares de $A$. Veremos que, neste caso, a definição de anel de frações é ligeiramente diferente.

Os resultados aqui enunciados podem ser encontrados em [10].

Definição 4.2.1. Sejam $A$ um anel e $S \subseteq A$ um subconjunto multiplicativo formado apenas por elementos regulares de A. Um anel de frações à esquerda (ou anel quociente de Ore à esquerda) para $A$ com respeito a $S$ é um anel $B$, contendo $A$ como subanel, tal que:

(a) cada elemento de $S$ é inversivel em $B, e$

(b) cada elemento de $B$ pode ser expresso na forma $s^{-1}$ a, para alguns a $\in A$ e $s \in S$.

Anéis de frações à direita são definidos de modo análogo, usando a forma $a^{-1}$.

Sejam $A$ um anel e $S \subseteq A$ um conjunto de Ore formado apenas por elementos regulares de $A$. Explicitaremos a construção de um anel de frações à esquerda de $A$, com respeito a $S$. Em seguida, mostraremos que o novo anel será também um anel de frações à direita de $A$.

(1) Definimos em $A \times S$ uma relação $\sim$ como segue:

$$
\left(a_{1}, s_{1}\right) \sim\left(a_{2}, s_{2}\right) \Longleftrightarrow \text { existem } c, d \in A \text { tais que } c a_{1}=d a_{2} \text { e } c s_{1}=d s_{2} \in S
$$

para todo $a_{1}, a_{2} \in A$ e $s_{1}, s_{2} \in S$. Então, $\sim$ é uma relação de equivalência.

Observação 4.2.2. Sejam $(a, s),(b, t) \in A \times S$ tais que $(a, s) \sim(b, t)$. Então, se existirem $c^{\prime}, d^{\prime} \in A$ tais que $c^{\prime} s=d^{\prime} t \in S$, teremos $c^{\prime} a=d^{\prime} b$.

Demonstração. Existem $c, d \in A$ tais que $c a=d b$ e $c s=d t \in S$; existem $c^{\prime}, d^{\prime} \in A$ tais que $c^{\prime} s=d^{\prime} t \in S$; pelas propriedades de $S$, existem $e \in A$ e $e^{\prime} \in S$ tais que $e(c s)=e^{\prime}\left(c^{\prime} s\right)$, de onde $e(d t)=e^{\prime}\left(d^{\prime} t\right)$; como $S$ possui apenas elementos regulares de $A$, então $e c=e^{\prime} c^{\prime}$ e $e d=e^{\prime} d^{\prime}$. Assim,

$$
\left(e^{\prime} c^{\prime}\right) a=(e c) a=e(c a)=e(d b)=(e d) b=\left(e^{\prime} d^{\prime}\right) b \Longrightarrow c^{\prime} a=d^{\prime} b .
$$

Demonstração. ( é uma relação de equivalência) 
(a) $\sim$ é reflexiva, pois para todo $t \in S$, ta $=t a$ e $t s=t s \in S$, para todo $a \in A$ e $s \in S$, já que $\mathrm{S}$ é um conjunto multiplicativo.

(b) Claramente, $\sim$ é simétrica.

(c) Sejam $a, b, c \in A$, e $s, t, u \in S$, $\operatorname{com}(a, s) \sim(b, t) \sim(c, u)$.

$$
\begin{aligned}
& (a, s) \sim(b, t) \Longrightarrow \exists u, u^{\prime} \in A \text { tais que } u a=u^{\prime} b \text { e } u s=u^{\prime} t \in S \\
& (b, t) \sim(c, u) \Longrightarrow \exists v, v^{\prime} \in A \text { tais que } v b=v^{\prime} \text { c e } v t=v^{\prime} u \in S
\end{aligned}
$$

Existem $z \in S$ e $z^{\prime} \in A$ tais que $z\left(u^{\prime} t\right)=z^{\prime}(v t) \Longrightarrow\left(z u^{\prime}\right) t=\left(z^{\prime} v\right) t \Longrightarrow z u^{\prime}=z^{\prime} v$, pois $t$ é um elemento regular. Assim,

$$
z(u s)=z\left(u^{\prime} t\right)=\left(z u^{\prime}\right) t=\left(z^{\prime} v\right) t=z^{\prime}(v t)=z^{\prime}\left(v^{\prime} u\right) \in S,
$$

pois $z(u s) \in S$. Concluímos que $\exists d, e, f \in A$ tais que $d s=e t=f u \in S$. Usando a Observação $4.2 .2, d a=e b=f c \Longrightarrow(a, s) \sim(c, u) ; \sim$ é transitiva.

Para cada $(a, s) \in A \times S$, denotaremos a classe de equivalência de $\sim$ que contém $(a, s)$ por $s^{-1} a$ e denotaremos por $B$ o conjunto de todas essas classes de equivalência.

(2) Definimos uma adição em $B$.

Como $S$ é um conjunto de Ore à esquerda, existem $c, d \in A$ tais que $c s=d t=s^{\prime} \in S$, para todo $s, t \in S$. Definimos:

$$
s^{-1} a+t^{-1} b:=s^{-1}(c a+d b)
$$

para todo $a, b \in A, s, t \in S$.

Notemos que, se $s=t$, então $s^{-1} a+t^{-1} b=s^{-1}(a+b)$.

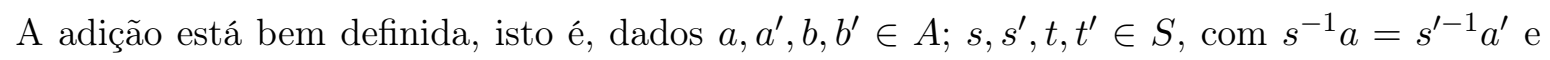
$t^{-1} b=t^{\prime-1} b^{\prime}$, então $s^{-1} a+t^{-1} b=s^{\prime-1} a^{\prime}+t^{\prime-1} b^{\prime}$.

Demonstração. Existem $c, d \in A$ tais que $c s=d t \in S$ e $c^{\prime}, d^{\prime} \in A$ tais que $c^{\prime} s^{\prime}=d^{\prime} t^{\prime} \in S$;e existem também $x, y \in A$ tais que $x(c s)=y\left(c^{\prime} s^{\prime}\right) \in S$, e $x(d t)=y\left(d^{\prime} t^{\prime}\right) \in S$.

$$
\begin{aligned}
& x(c s)=y\left(c^{\prime} s^{\prime}\right) \in S \Longrightarrow(x c) a=\left(y c^{\prime}\right) a^{\prime} \mathrm{e} \\
& x(d t)=y\left(d^{\prime} t^{\prime}\right) \in S \Longrightarrow(x d) b=\left(y d^{\prime}\right) b^{\prime}
\end{aligned}
$$

pela Observação 4.2.2. Assim, $x(c a+d b)=y\left(c^{\prime} a^{\prime}+d^{\prime} b^{\prime}\right)$, e segue o resultado. 
Se existirem $u, v \in A$ tais que $u s=v t \in S$, como

$$
(c s)^{-1} c a=s^{-1} a=(u s)^{-1} u a \text { e }(d t)^{-1} d b=t^{-1} b=(v t)^{-1} v b
$$

a escolha do par não afetará a adição, já que a mesma está bem definida.

(3) Definimos uma multiplicação em $B$.

Como $S$ satisfaz a condição de Ore à esquerda, existem $c \in S$ e $d \in A$ tais que $c a=d t$, para todo $a \in A$ e $t \in S$. Definimos:

$$
\left(s^{-1} a\right)\left(t^{-1} b\right):=(c s)^{-1} d b,
$$

para todo $a, b \in A ; s, t \in S$.

A multiplicação está bem definida, isto é, dados $a, a^{\prime}, b, b^{\prime} \in A ; s, s^{\prime}, t, t^{\prime} \in S$, com $s^{-1} a=s^{\prime-1} a^{\prime}$ e $t^{-1} b=t^{\prime-1} b^{\prime}$, então $\left(s^{-1} a\right)\left(t^{-1} b\right)=\left(s^{\prime-1} a^{\prime}\right)\left(t^{\prime-1} b^{\prime}\right)$.

Demonstração. Existem $u \in S$ e $u^{\prime} \in A$ tais que $u t=u^{\prime} t^{\prime} \in S \Longrightarrow u b=u^{\prime} b^{\prime}$, pela Observação 4.2.2; existem também $v \in S$ e $c \in A$ tais que $v a=c(u t)=(c u) t$ e $v^{\prime} \in S, c^{\prime} \in A$ tais que $v^{\prime} a^{\prime}=c^{\prime}\left(u^{\prime} t^{\prime}\right)=\left(c^{\prime} u^{\prime}\right) t^{\prime}$.

Com as igualdades que temos, aplicando a definição da multiplicação em $B$, segue que

$$
\left(s^{-1} a\right)\left(t^{-1} b\right)=(v s)^{-1} c u b \text { e }\left(s^{\prime-1} a^{\prime}\right)\left(t^{-1} b^{\prime}\right)=\left(v^{\prime} s^{\prime}\right)^{-1} c^{\prime} u^{\prime} b^{\prime} .
$$

Precisamos então mostrar que $(v s)^{-1} c u b=\left(v^{\prime} s^{\prime}\right)^{-1} c^{\prime} u^{\prime} b^{\prime}$; existem $x \in S$ e $x^{\prime} \in A$ tais que $x(v s)=x^{\prime}\left(v^{\prime} s^{\prime}\right) \in S$. Pela Observação 4.2.2, temos $(x v) a=\left(x^{\prime} v^{\prime}\right) a^{\prime}$, já que $s^{-1} a=s^{\prime-1} a^{\prime}$.

$$
x(v a)=x^{\prime}\left(v^{\prime} a^{\prime}\right) \Longrightarrow x(c u t)=x^{\prime}\left(c^{\prime} u^{\prime} t^{\prime}\right) \Longrightarrow x c=x^{\prime} c^{\prime},
$$

pois $S$ possui apenas elementos regulares de $A$.

Então, $(x c)(u b)=\left(x^{\prime} c^{\prime}\right)\left(u^{\prime} b^{\prime}\right) \Longrightarrow x(c u b)=x^{\prime}\left(c^{\prime} u^{\prime} b^{\prime}\right) \Longrightarrow(c u b, v s) \sim\left(c^{\prime} u^{\prime} b^{\prime}, v^{\prime} s^{\prime}\right)$, o que nos dá a igualdade desejada.

Se existirem $c, c^{\prime} \in S$ e $d, d^{\prime} \in A \operatorname{com} c a=d t$ e $c^{\prime} a=d^{\prime} t$, então

$$
\left(s^{-1} a\right)\left(t^{-1} b\right)=(c s)^{-1} d b=\left(c^{\prime} s\right)^{-1} d^{\prime} b,
$$

para todo $a, b \in A ; s, t \in S$.

Demonstração. Existem $x \in S$ e $x^{\prime} \in A$ tais que $x c=x^{\prime} c^{\prime} \in S$, de onde $(x c) s=\left(x^{\prime} c^{\prime}\right) s \in S$; $(x c) a=\left(x^{\prime} c^{\prime}\right) a \Longrightarrow x(d t)=x^{\prime}\left(d^{\prime} t\right)$, de onde $x d=x^{\prime} d^{\prime}$, pois $t$ é um elemento regular. Assim, $(x d) b=\left(x^{\prime} d^{\prime}\right) b$ e, portanto, $(d b, c s) \sim\left(d^{\prime} b, c^{\prime} s\right)$, como queremos. 
(4) O conjunto $B$ munido das operações de adição e multiplicação definidas nos passos anteriores é um anel associativo (não necessariamente comutativo), com elemento identidade, e no qual os elementos da forma $1^{-1} s$ são inversíveis, para todo $s \in S$.

(a) Notemos que a adição em $B$ é comutativa, pois $s^{-1} a+s^{-1} b=s^{-1} b+s^{-1} a$, para todo $a, b \in A, s \in S$.

(b) Para todo $a, b, c \in A ; s \in S$,

$$
\begin{aligned}
\left(s^{-1} a+s^{-1} b\right)+s^{-1} c & =s^{-1}(a+b)+s^{-1} c=s^{-1}((a+b)+c) \\
& =s^{-1}(a+(b+c))=s^{-1} a+s^{-1}(b+c) \\
& =s^{-1} a+\left(s^{-1} b+s^{-1} c\right),
\end{aligned}
$$

e pelo Lema A.1.3 temos a associatividade da adição.

(c) Para todo $a \in A$ e $s \in S$, existem $c, d \in A$ tais que $c s=d 1=d \in S$, devido às propriedades de $S$.

$$
s^{-1} a+1^{-1} 0=d^{-1}(c a+d 0)=d^{-1}(c a+0)=d^{-1}(c a)=(c s)^{-1} c a=s^{-1} a .
$$

(d) Claramente, para todo $a \in A, s \in S, s^{-1}(-a)$ é o oposto aditivo de $s^{-1} a$ em $B$, em que $(-a)$ é o oposto aditivo de $a$ em $A$.

(e) Para todo $s^{-1} a, t^{-1} b, u^{-1} c \in B,\left(\left(s^{-1} a\right)\left(t^{-1} b\right)\right) u^{-1} c=s^{-1} a\left(\left(t^{-1} b\right)\left(u^{-1} c\right)\right)$. De fato, pelas propriedades de $S$, existem $d \in A, v \in S$ tais que $d u=v b ; e \in A, w \in S$ tais que $w a=e(v t)=(e v) t$

$$
s^{-1} a\left(\left(t^{-1} b\right)\left(u^{-1} c\right)\right)=s^{-1} a\left((v t)^{-1} d c\right)=(w s)^{-1} e d c
$$

$$
\begin{aligned}
w a & =(e v) t \Longrightarrow\left(s^{-1} a\right)\left(t^{-1} b\right)=(w s)^{-1} e v b \Longrightarrow\left(\left(s^{-1} a\right)\left(t^{-1} b\right)\right) u^{-1} c=\left((w s)^{-1} e v b\right) u^{-1} c \\
d u & =v b \Longrightarrow e(d u)=(e d) u=e(v b) \Longrightarrow\left((w s)^{-1} e v b\right) u^{-1} c=(w s)^{-1} e d c,
\end{aligned}
$$

e temos a igualdade desejada.

(f) Para todo $a \in A$ e $s \in S$, pelas propriedades de $S$, existem $c \in A, d \in S$ tais que $d a=c 1$; assim, $\left(s^{-1} a\right)\left(1^{-1} 1\right)=(d s)^{-1} c 1=(d s)^{-1} d a=s^{-1} a$. Analogamente, existem $c^{\prime} \in A$, $d^{\prime} \in S$ tais que $d^{\prime}=d^{\prime} 1=c^{\prime} s$, e temos $\left(1^{-1} 1\right)\left(s^{-1} a\right)=\left(d^{\prime} 1\right)^{-1} c^{\prime} a=\left(c^{\prime} s\right)^{-1} c^{\prime} a=s^{-1} a$. 
Logo, $1^{-1} 1$ é o elemento identidade de $B$.

(g) Para todo $s \in S,\left(1^{-1} s\right)\left(s^{-1} 1\right)=t^{-1} t=1^{-1} 1$; e $\left(s^{-1} 1\right)\left(1^{-1} s\right)=(t s)^{-1} t s=1^{-1} 1$, para todo $t \in S$.

Notemos que para todo $a \in A$ e todo $s, t \in S,\left(s^{-1} 1\right)\left(1^{-1} a\right)=(t s)^{-1} t a=s^{-1} a$.

(h) Para mostrar as leis distributivas em $B$, usaremos que $\left(s^{-1} 1\right)\left(1^{-1} a\right)=s^{-1} a$, para todo $a \in A$ e $s \in S$.

Para mostrar a lei à esquerda, é suficiente mostrar que

$$
1^{-1} a\left(t^{-1} b+t^{-1} c\right)=\left(1^{-1} a\right)\left(t^{-1} b\right)+\left(1^{-1} a\right)\left(t^{-1} c\right)
$$

para todo $a, b, c \in A$ e $t \in S$.

Demonstração. Existem $u \in A$ e $v \in S$ tais que $v a=u t$ e então

$$
\begin{aligned}
1^{-1} a\left(t^{-1}(b+c)\right) & =v^{-1}(u(b+c))=v^{-1}(u b+u c)=v^{-1}(u b)+v^{-1}(u c) \\
& =\left(1^{-1} a\right)\left(t^{-1} b\right)+\left(1^{-1} a\right)\left(t^{-1} c\right) .
\end{aligned}
$$

Para mostrar a lei à direita, mostraremos que

$$
\left(t^{\prime-1}\left(b^{\prime}+c^{\prime}\right)\right) 1^{-1} f=\left(t^{\prime-1} b^{\prime}\right)\left(1^{-1} f\right)+\left(t^{\prime-1} c^{\prime}\right)\left(1^{-1} f\right),
$$

para todo $b^{\prime}, c^{\prime}, f \in A$ e $t^{\prime} \in S$.

Demonstração. Existem $u^{\prime} \in A$ e $v^{\prime} \in S$ tais que $u^{\prime} 1=v^{\prime}\left(b^{\prime}+c^{\prime}\right)$ e

$$
\begin{aligned}
\left(t^{\prime-1}\left(b^{\prime}+c^{\prime}\right)\right) 1^{-1} f & =\left(v^{\prime} t^{\prime}\right)^{-1} u^{\prime} f=\left(v^{\prime} t^{\prime}\right)^{-1}\left(v^{\prime}\left(b^{\prime}+c^{\prime}\right)\right) f=\left(v^{\prime} t^{\prime}\right)^{-1} v^{\prime}\left(\left(b^{\prime}+c^{\prime}\right) f\right) \\
& =t^{\prime-1}\left(\left(b^{\prime}+c^{\prime}\right) f\right)=\left(t^{\prime-1} b^{\prime}\right)\left(1^{-1} f\right)+\left(t^{\prime-1} c^{\prime}\right)\left(1^{-1} f\right) .
\end{aligned}
$$

Proposição 4.2.3. A aplicação $f: A \longrightarrow B$, dada por $f(a)=1^{-1}$ a define um isomorfismo de $A$ em um subanel de B; e quando $A$ é identificado com esse subanel, $B$ torna-se o anel de frações à esquerda de $A$ com respeito a $S$.

Demonstração. (a) $f$ é um homomorfismo de anéis. De fato, para todo $a, b \in A$,

$$
\begin{aligned}
f(a+b) & =1^{-1}(a+b)=1^{-1} a+1^{-1} b=f(a)+f(b) \mathrm{e} \\
f(a b) & =1^{-1}(a b)=\left(1^{-1} a\right)\left(1^{-1} b\right)=f(a) f(b) ;
\end{aligned}
$$

(b) Seja $a \in A$ tal que $f(a)=1^{-1} a=1^{-1} 0$; pela Observação $4.2 .2, a=1 a=0$, de onde segue 
que $f$ é injetiva. $A$ será então isomorfo a $f(A) \subseteq B$, que é um subanel de $B$.

A partir de agora, denotaremos $B$ por $S^{-1} A$. Após a identificação de $A$ com um subanel de $S^{-1} A$, podemos afirmar que cada elemento de $S$ é inversível em $S^{-1} A$; e denotaremos esse inverso por $s^{-1}$, para todo $s \in S$;

De forma análoga, podemos construir o anel de frações à direita de $A$, denotado por $A S^{-1}$, a partir do mesmo conjunto $S \subseteq A$, que é também um conjunto de Ore à direita.

Proposição 4.2.4. Sejam $A$ um anel $e S \subseteq A$ um conjunto de Ore, formado apenas por elementos regulares de $A$. Então, $A S^{-1}=S^{-1} A$, isto é, um anel de frações à direita de $A$ com respeito a $S$ é também um anel de frações à esquerda de $A$ com respeito a $S$ e vice-versa.

Demonstração. Como $S^{-1} A$ é um anel de frações à esquerda de $A$ com respeito a $S$, temos que $A \subseteq S^{-1} A$ como subanel e todos os elementos de $S$ são inversíveis em $S^{-1} A$, e ainda que todo elemento de $S^{-1} A$ pode ser escrito da forma $s^{-1} a$, para algum $a \in A$ e $s \in S$. Como $S$ é um conjunto de Ore à direita, existem $b \in A$ e $t \in S$ tais que

$$
\begin{aligned}
a t=s b & \Longrightarrow s^{-1} a=b t^{-1} \\
& \Longrightarrow s^{-1} a \in A S^{-1} \Longrightarrow S^{-1} A \subseteq A S^{-1} .
\end{aligned}
$$

De modo análogo, mostra-se que $A S^{-1} \subseteq S^{-1} A$.

Podemos então concluir que $S^{-1} A=A S^{-1}$ satisfaz as condições da Definição 4.2.1.

Nossa abordagem para mostrar a existência de um anel de frações à esquerda (respectivamente à direita) de $A$ foi totalmente construtiva. Esta, porém, não é a única forma possível, conforme o teorema que enunciaremos a seguir.

Teorema 4.2.5 (Ore,Asano). Sejam $A$ um anel e $S \subseteq A$ um conjunto multiplicativo formado apenas por elementos regulares. Então, existe um anel de frações à esquerda (respectivamente à direita) de A com respeito a $S$ se e somente se $S$ é um conjunto de Ore à esquerda (respectivamente à direita).

(vide [10, p.108-109]).

Neste ponto, surge naturalmente o questionamento sobre a unicidade do anel de frações à esquerda (respectivamente à direita) de $A$. A proposição e o corolário abaixo fornecem a unicidade utilizando a abordagem não construtiva, e o resultado é válido quando $S$ é um conjunto de Ore à esquerda formado apenas por elementos regulares. Como sempre, resultado análogo pode ser enunciado para conjuntos de Ore à direita e anéis de frações à direita.

Proposição 4.2.6. Sejam $A$ um anel e $S \subseteq A$ um conjunto de Ore à esquerda de A, formado apenas por elementos regulares. Seja $S^{-1} A$ o anel de frações à esquerda de $A$ com respeito a $S$. Suponha que $\phi: A \longrightarrow T$ seja um homomorfismo de anéis tal que $\phi(s)$ é inversivel em $T$, para todo $s \in S$. Então, $\phi$ estende-se unicamente a um homomorfismo de anéis $\psi: S^{-1} A \longrightarrow T$.

(vide [10, p.110]). 
Corolário 4.2.7. Sejam $A$ um anel $e S \subseteq A$ um conjunto de Ore à esquerda formado apenas por elementos regulares. Sejam $\left(S^{-1} A\right)_{1} e\left(S^{-1} A\right)_{2}$ anéis de fraçôes à esquerda de $A$ com respeito a $S$. Então, a aplicação identidade de $A$ estende-se unicamente a um isomorfismo de $\left(S^{-1} A\right)_{1}$ em $\left(S^{-1} A\right)_{2}$.

Demonstração. Suponha que $\left(S^{-1} A\right)_{1}$ e $\left(S^{-1} A\right)_{2}$ sejam anéis de frações à esquerda de $A$. Então, como $A$ pode ser identificado com um subanel de $\left(S^{-1} A\right)_{2}$, podemos aplicar a Proposição 4.2 .6 com $\phi: A \longrightarrow\left(S^{-1} A\right)_{2}$ sendo a aplicação inclusão de $A$ em $\left(S^{-1} A\right)_{2}$. Assim, existe um único homomorfismo de anéis $\psi_{1}:\left(S^{-1} A\right)_{1} \longrightarrow\left(S^{-1} A\right)_{2}$ tal que $\psi_{1}\left\lceil_{A}=\phi\right.$, ou seja, podemos dizer que $\psi_{1}$ restrito a $A$ é a aplicação identidade de $A$. De modo análogo, existe um único homomorfismo de anéis $\psi_{2}:\left(S^{-1} A\right)_{2} \longrightarrow\left(S^{-1} A\right)_{1}$ tal que $\psi_{2}$ restrito a $A$ é a aplicação identidade de $A$. Logo, $\psi_{2} \circ \psi_{1}:\left(S^{-1} A\right)_{1} \longrightarrow\left(S^{-1} A\right)_{1}$ é um homomorfismo de anéis tal que $\left(\psi_{2} \circ \psi_{1}\right) \uparrow_{A}$ é a aplicação identidade de $A$. Mas a aplicação identidade de $\left(S^{-1} A\right)_{1}$ possui a mesma propriedade. Na verdade, aplicando novamente a Proposição $4.2 .6 \mathrm{com} T=\left(S^{-1} A\right)_{1}$, temos que é a única aplicação de $\left(S^{-1} A\right)_{1}$ em $\left(S^{-1} A\right)_{1}$ com essa propriedade. De modo análogo, $\psi_{1} \circ \psi_{2}$ é a aplicação identidade de $\left(S^{-1} A\right)_{2}$. Assim, $\psi_{1}$ e $\psi_{2}$ são isomorfismos inversos um do outro, e temos o resultado desejado.

Para o caso geral, em que $S$ não é necessariamente um conjunto formado apenas por elementos regulares, temos uma definição ligeiramente diferente para anéis de frações à esquerda (respectivamente à direita) de $A$ :

Definição 4.2.8. Sejam $A$ um anel $e S \subseteq A$ um conjunto multiplicativo. Um anel de frações à esquerda (ou anel quociente de Ore à esquerda, ou localização de Ore à esquerda) de A com respeito a $S$ é um homomorfismo de anéis $\phi: A \longrightarrow B$ tal que:

(a) $\phi(s)$ é uma unidade de $B$, para todo $s \in S$;

(b) Cada elemento de $B$ tem a forma $\phi(s)^{-1} \phi(a)$, para alguns a $\in A$ e $s \in S$;

(c) $\operatorname{Ker} \phi=\{a \in A \mid s a=0$, para algum $s \in S\}$.

Notemos que aqui, como há a possibilidade de encontrarmos divisores de zero em $S, \operatorname{Ker} \phi$ não precisa ser trivial, e por isso não temos necessariamente a imersão de $A$ em $B$ como subanel.

De modo análogo, definimos localização de Ore à direita de $A$ com respeito a $S$, quando $S$ é um conjunto de Ore à direita, utilizando $\phi(a) \phi(s)^{-1}$, para $a \in A$ e $s \in S$.

Para que seja enunciado o teorema que trata do caso geral, necessitamos de algumas definições adicionais:

Definição 4.2.9. Seja $S$ um conjunto multiplicativo em um anel A. Então, $S$ é anulável à esquerda se e somente se para $a \in A$ e $s \in S$ tais que as $=0$, existe $s^{\prime} \in S$ tal que $s^{\prime} a=0$. Um conjunto denominador à esquerda é um conjunto de Ore à esquerda, que é anulável à esquerda.

De modo análogo, definimos conjunto anulável à direita e conjunto denominador à direita. Um conjunto denominador é um conjunto que é simultaneamente denominador à direita e à esquerda.

Teorema 4.2.10 (Gabriel). Sejam $A$ um anel e $S \subseteq A$ um conjunto multiplicativo. Então, existe um anel de frações à esquerda (respectivamente à direita) para $A$ com respeito a $S$ se e somente se $S$ é um conjunto denominador à esquerda (respectivamente à direita). 
(vide [10, p.169-170]).

A proposição e o Corolário abaixo fornecem a unicidade no caso de um anel de frações à esquerda. Mas o análogo para um anel de frações à direita também é válido.

Proposição 4.2.11. Sejam $A$ um anel $e S \subseteq A$ um conjunto denominador à esquerda. Considere $\phi: A \longrightarrow B$ um anel de frações à esquerda de $A$ com respeito a $S$. Se $\psi: A \longrightarrow T$ é um homomorfismo de anéis tal que $\psi(s)$ é inversivel em $T$ para todo $s \in S$, então existe um único homomorfismo de anéis $\eta: B \longrightarrow T$ tal que $\psi=\eta \circ \phi$.

(vide [10, p.170]).

Usando os argumentos clássicos para objetos com propriedades universais, mostra-se então a unicidade:

Corolário 4.2.12. Sejam $A$ um anel $e S \subseteq A$ um conjunto denominador à esquerda, e suponha que $\phi_{1}: A \longrightarrow B_{1}$ e $\phi_{2}: A \longrightarrow B_{2}$ são anéis de frações à esquerda de $A$ com respeito a $S$. Então, existe um único isomorfismo $\eta: B_{1} \longrightarrow B_{2}$ tal que $\eta \circ \phi_{1}=\phi_{2}$.

\subsubsection{Módulos sobre Anéis não Comutativos}

Seja $M$ um $A$-módulo à esquerda. Nesta subseção, construiremos um $S^{-1} A$-módulo à esquerda, denominado módulo de frações de $M$ com respeito a $S$. Simetricamente, podemos repetir a construção para módulos à direita, a partir de conjuntos de Ore à direita.

Denotaremos tanto o elemento neutro da adição do anel, como o de um módulo sobre esse anel apenas por 0 .

Definição 4.2.13. Sejam $A$ um anel $e S \subseteq A$ um conjunto de Ore à esquerda formado apenas por elementos regulares de $A$. Considere $M$ um A-módulo à esquerda. Um módulo de frações para $M$ com respeito a $S$ consiste de um $S^{-1} A$-módulo $M^{\prime}$ à esquerda, junto com um homomorfismo de A-módulos $\psi: M \longrightarrow M^{\prime}$ tal que:

(a) Cada elemento de $M^{\prime}$ tem a forma $s^{-1} \psi(m)$, para algum $m \in M$ e $s \in S$;

(b) $\operatorname{Ker} \psi=\{m \in M \mid s m=0$, para algum $s \in S\}$.

Muitas vezes, por um abuso de notação, nos referimos a $M^{\prime}$ como módulo de frações de $M$, ao invés de citarmos o par $M^{\prime}, \psi$.

De modo análogo, quando $S$ for um conjunto de Ore à direita, definimos módulo de frações para um $A$-módulo $N$ à direita.

No caso geral, em que $S$ pode eventualmente possuir divisores de zero em $A$, exigimos que $S$ seja um conjunto denominador à esquerda (respectivamente à direita), ao invés de apenas um conjunto de Ore à esquerda (respectivamente à direita) na Definição 4.2.13.

A seguir, construiremos o módulo de frações para $M$, em que $M$ é um módulo à esquerda de $A$.

(1) Definimos uma relação $\sim$ em $M \times S$, como segue:

$$
\left(m_{1}, s_{1}\right) \sim\left(m_{2}, s_{2}\right) \Longleftrightarrow \exists c, d \in A \text { tais que } c m_{1}=d m_{2} \text { e } c s_{1}=d s_{2} \in S,
$$

para todo $m_{1}, m_{2} \in M$ e $s_{1}, s_{2} \in S$. Então, $\sim$ é uma relação de equivalência. 
Demonstração. (a) Notemos que $\sim$ é reflexiva, pois para todo $t \in S, t m=t m$ e $t s=t s \in S$, para todo $m \in M, s \in S$, já que $S$ é um conjunto multiplicativo.

(b) Claramente, $\sim$ é simétrica.

(c) Sejam $m_{1}, m_{2}, m_{3} \in M, s_{1}, s_{2}, s_{3} \in S \operatorname{com}\left(m_{1}, s_{1}\right) \sim\left(m_{2}, s_{2}\right) \sim\left(m_{3}, s_{3}\right)$

$$
\begin{aligned}
& \left(m_{1}, s_{1}\right) \sim\left(m_{2}, s_{2}\right) \Longrightarrow \exists u, u^{\prime} \in A \text { tais que } u m_{1}=u^{\prime} m_{2} \text { e } u s_{1}=u^{\prime} s_{2} \in S \\
& \left(m_{2}, s_{2}\right) \sim\left(m_{3}, s_{3}\right) \Longrightarrow \exists v, v^{\prime} \in A \text { tais que } v m_{2}=v^{\prime} m_{3} \text { e } v s_{2}=v^{\prime} s_{3} \in S
\end{aligned}
$$

$\exists z \in S$ e $z^{\prime} \in A$ tais que $z\left(u^{\prime} s_{2}\right)=z^{\prime}\left(v s_{2}\right) \Longrightarrow\left(z u^{\prime}\right) s_{2}=\left(z^{\prime} v\right) s_{2} \Longrightarrow z u^{\prime}=z^{\prime} v$

pois $S$ possui apenas elementos regulares de $A$. Assim,

$$
z\left(u s_{1}\right)=z\left(u^{\prime} s_{2}\right)=\left(z u^{\prime}\right) s_{2}=\left(z^{\prime} v\right) s_{2}=z^{\prime}\left(v s_{2}\right)=z^{\prime}\left(v^{\prime} s_{3}\right) \in S
$$

pois $z\left(u s_{1}\right) \in S$, já que $S$ é um conjunto multiplicativo.

De modo análogo,

$$
\begin{aligned}
& z\left(u m_{1}\right)=z\left(u^{\prime} m_{2}\right)=\left(z u^{\prime}\right) m_{2}=\left(z^{\prime} v\right) m_{2}=z^{\prime}\left(v m_{2}\right)=z^{\prime}\left(v^{\prime} m_{3}\right) \Longrightarrow(z u) m_{1}=\left(z^{\prime} v^{\prime}\right) m_{3}, \\
& \text { e } \sim \text { é transitiva. }
\end{aligned}
$$

Para cada $(m, s) \in M \times S$, denotaremos a classe de equivalência de $\sim$ que contém $(m, s)$ por $s^{-1} m$ e o conjunto de todas essas classes por $S^{-1} M$.

(2) Definimos uma adição em $S^{-1} M$.

Sejam $m_{1}, m_{2} \in M$ e $s_{1}, s_{2} \in S$. Como $S$ é um conjunto de Ore à esquerda, existem $c, d \in A$ tais que $c s_{1}=d s_{2}=t \in S$. Definimos:

$$
s_{1}^{-1} m_{1}+s_{2}^{-1} m_{2}:=t^{-1}\left(c m_{1}+d m_{2}\right)
$$

No caso particular em que $s_{1}=s_{2}, s_{1}^{-1} m_{1}+s_{1}^{-1} m_{2}=s_{1}^{-1}\left(m_{1}+m_{2}\right)$.

É necessário mostrar que a adição está bem definida, isto é, dados $m_{1}, m_{2}, m_{3}, m_{4} \in M$ e 
$s_{1}, s_{2}, s_{3}, s_{4} \in S$, com $s_{1}^{-1} m_{1}=s_{3}^{-1} m_{3}$ e $s_{2}^{-1} m_{2}=s_{4}^{-1} m_{4}$, então

$$
s_{1}^{-1} m_{1}+s_{2}^{-1} m_{2}=s_{3}^{-1} m_{3}+s_{4}^{-1} m_{4}
$$

Mostraremos que a adição não depende da escolha do representante da classe de $s_{1}^{-1} m_{1}$.

De modo análogo, é possível mostrar que também não depende da escolha do representante da classe de $s_{2}^{-1} m_{2}$.

Demonstração. Em primeiro lugar, pela definição de adição,

$$
s_{1}^{-1} m_{1}+s_{2}^{-1} m_{2}=\left(\tilde{r}_{1} s_{1}\right)^{-1}\left(\tilde{r}_{1} m_{1}+\tilde{r}_{2} m_{2}\right)
$$

para $\tilde{r}_{1}, \tilde{r}_{2} \in A$, tais que $\tilde{r}_{1} s_{1}=\tilde{r}_{2} s_{2} \in S$.

Por outro lado, como $s_{1}^{-1} m_{1}=s_{3}^{-1} m_{3}$, existem $r, s \in A$ tais que $r s_{1}=s s_{3} \in S$ e $r m_{1}=s m_{3}$. Assim, $s_{1}^{-1} m_{1}=\left(r s_{1}\right)^{-1} r m_{1}=\left(s s_{3}\right)^{-1} s m_{3}=s_{3}^{-1} m_{3}$.

Como $S$ é um conjunto de Ore à esquerda, existem $\tilde{r}_{3} \in S$ e $\tilde{r}_{4} \in A$ tais que $\tilde{r}_{3} r s_{1}=\tilde{r}_{4} \tilde{r}_{1} s_{1} \in S$. Então, $\tilde{r}_{3} r s_{1}=\tilde{r}_{4} \tilde{r}_{1} s_{1}=\tilde{r}_{4} \tilde{r}_{2} s_{2} \in S$. Como $S$ é formado apenas por elementos regulares, temos ainda $\tilde{r}_{3} r=\tilde{r}_{4} \tilde{r}_{1}$. Portanto,

$$
\begin{aligned}
\left(r s_{1}\right)^{-1} r m_{1}+s_{2}^{-1} m_{2} & =\left(\tilde{r}_{3} r s_{1}\right)^{-1}\left(\tilde{r}_{3} r m_{1}+\tilde{r}_{4} \tilde{r}_{2} m_{2}\right) \\
& =\left(\tilde{r}_{4} \tilde{r}_{1} s_{1}\right)^{-1}\left(\tilde{r}_{4} \tilde{r}_{1} m_{1}+\tilde{r}_{4} \tilde{r}_{2} m_{2}\right)=\left(\tilde{r}_{1} s_{1}\right)^{-1}\left(\tilde{r}_{1} m_{1}+\tilde{r}_{2} m_{2}\right),
\end{aligned}
$$

o que fornece a igualdade desejada.

Se existirem $u, v \in A$ tais que $u s_{1}=v s_{2} \in S$, veremos que a adição não depende da escolha do par:

Demonstração. Existem $r_{3} \in A, r_{4} \in S$ tais que $r_{3} \tilde{r}_{1} s_{1}=r_{4} u s_{1} \in S$, pois $S$ é um conjunto de Ore à esquerda. Então, $r_{3} \tilde{r}_{1} s_{1}=r_{4} u s_{1}=r_{4} v s_{2} \in S$. Como $S$ possui apenas elementos regulares, temos ainda que $r_{3} \tilde{r}_{1}=r_{4} u$. Assim,

$$
\left(\tilde{r}_{1} s_{1}\right)^{-1}\left(\tilde{r}_{1} m_{1}+\tilde{r}_{2} m_{2}\right)=\left(r_{3} \tilde{r}_{1} s_{1}\right)^{-1}\left(r_{3} \tilde{r}_{1} m_{1}+r_{3} \tilde{r}_{2} m_{2}\right)=\left(r_{4} u s_{1}\right)^{-1}\left(r_{4} u m_{1}+r_{3} \tilde{r}_{2} m_{2}\right)
$$

Por outro lado, $r_{3} \tilde{r}_{2} s_{2}=r_{3} \tilde{r}_{1} s_{1}=r_{4} u s_{1}=r_{4} v s_{2}$, o que nos dá $r_{3} \tilde{r}_{2}=r_{4} v$, pois $S$ possui apenas elementos regulares. Assim, 


$$
\left(r_{4} u s_{1}\right)^{-1}\left(r_{4} u m_{1}+r_{3} \tilde{r}_{2} m_{2}\right)=\left(r_{4} u s_{1}\right)^{-1}\left(r_{4} u m_{1}+r_{4} v m_{2}\right)=\left(u s_{1}\right)^{-1}\left(u m_{1}+v m_{2}\right),
$$

como queríamos.

(3) $S^{-1} M$ é um grupo abeliano com relação à adição.

(a) A adição é comutativa;

(b) A adição é associativa;

(c) $s^{-1} 0$ é o elemento neutro aditivo em $S^{-1} M$, com $0 \in M$ e $1 \in S$; e

(d) Para todo $m \in M, s \in S, s^{-1}(-m)$ é o oposto aditivo de $s^{-1} m$, onde $(-m)$ é o oposto aditivo de $m$ em $M$.

A demonstração dos itens acima é análoga a que fizemos para demonstrar que $S^{-1} A$ é um grupo abeliano em relação à adição.

(4) Definimos uma lei de composição externa em $S^{-1} A \times S^{-1} M$ : para todo $m \in M, a \in A$, $s, t \in S$, existem $c \in A, d \in S$ tais que $d a=c t$ e

$$
s^{-1} a \cdot t^{-1} m:=(d s)^{-1} c m
$$

A lei de composição está bem definida:

Demonstração. Sejam $m_{1}, m_{2} \in M, s_{1}, s_{2}, t_{1}, t_{2} \in S, a_{1}, a_{2} \in A$, com $s_{1}^{-1} a_{1}=s_{2}{ }^{-1} a_{2}$ e $t_{1}^{-1} m_{1}=t_{2}^{-1} m_{2}$; existem $u_{1}, u_{2} \in A$ tais que $u_{1} m_{1}=u_{2} m_{2}$ e $u_{1} t_{1}=u_{2} t_{2} \in S$, e $v_{1}, v_{2} \in S$, $c_{1}, c_{2} \in A$ tais que $v_{1} a_{1}=c_{1}\left(u_{1} t_{1}\right)$ e $v_{2} a_{2}=c_{2}\left(u_{2} t_{2}\right)$.

$$
\begin{aligned}
& s_{1}{ }^{-1} a_{1} \cdot t_{1}{ }^{-1} m_{1}=\left(v_{1} s_{1}\right)^{-1}\left(c_{1} u_{1} m_{1}\right) \\
& s_{2}{ }^{-1} a_{2} \cdot t_{2}{ }^{-1} m_{2}=\left(v_{2} s_{2}\right)^{-1}\left(c_{2} u_{2} m_{2}\right)
\end{aligned}
$$

Existem $x_{1}, x_{2} \in A$ tais que $x_{1}\left(v_{1} s_{1}\right)=x_{2}\left(v_{2} s_{2}\right) \in S$. Pela Observação 4.2 .2 , temos

$$
\left(x_{1} v_{1}\right) a_{1}=\left(x_{2} v_{2}\right) a_{2} \Longrightarrow x_{1} c_{1} u_{1} t_{1}=x_{2} c_{2} u_{2} t_{2} \Longrightarrow x_{1} c_{1}=x_{2} c_{2} \Longrightarrow x_{1} c_{1} u_{1} m_{1}=x_{2} c_{2} u_{2} m_{2}
$$
de onde $s_{1}^{-1} a_{1} \cdot t_{1}^{-1} m_{1}=s_{2}^{-1} a_{2} \cdot t_{2}{ }^{-1} m_{2}$

Se existirem $c^{\prime} \in A, d^{\prime} \in S$ tais que $d^{\prime} a=c^{\prime} t$, então $(d s)^{-1} c m=\left(d^{\prime} s\right)^{-1} c^{\prime} m$. 
Demonstração. Existem $x_{1}, x_{2} \in A$ tais que $x_{1} d=x_{2} d^{\prime} \in S$, de onde $x_{1}(d s)=x_{2}\left(d^{\prime} s\right) \in S$. Assim,

$$
\begin{aligned}
\left(x_{1} d\right) a=\left(x_{2} d^{\prime}\right) a \Longrightarrow x_{1}(d a)=x_{2}\left(d^{\prime} a\right) & \Longrightarrow x_{1}(c t)=x_{2}\left(c^{\prime} t\right) \Longrightarrow x_{1} c=x_{2} c^{\prime} \\
& \Longrightarrow\left(x_{1} c\right) m=\left(x_{2} c^{\prime}\right) m \Longrightarrow x_{1}(c m)=x_{2}\left(c^{\prime} m\right) \\
& \Longrightarrow(d s)^{-1} c m=\left(d^{\prime} s\right)^{-1} c^{\prime} m .
\end{aligned}
$$

(5) $S^{-1} M$ é um $S^{-1} A$-módulo à esquerda, com respeito à lei de composição externa que acabamos de definir.

(a) Para todo $t \in S, m \in M, 1^{-1} 1 \cdot t^{-1} m=(t 1)^{-1} 1 m=t^{-1} m$.

(b) Para todo $a_{1}, a_{2} \in A, m \in M, s_{1}, s_{2}, t \in S$,

$$
\left(\left(s_{1}^{-1} a_{1}\right)\left(s_{2}^{-1} a_{2}\right)\right) \cdot t^{-1} m=s_{1}^{-1} a_{1} \cdot\left(s_{2}^{-1} a_{2} \cdot t^{-1} m\right)
$$

Demonstração. Existem $c \in A, d \in S$ tais que

$$
d a_{2}=c t \Longrightarrow s_{2}^{-1} a_{2} \cdot t^{-1} m=\left(d s_{2}\right)^{-1} c m
$$

existem $c^{\prime} \in A, d^{\prime} \in S$ tais que

$$
d^{\prime} a_{1}=c^{\prime}\left(d s_{2}\right) \Longrightarrow s_{1}{ }^{-1} a_{1} \cdot\left(d s_{2}\right)^{-1} c m=\left(d^{\prime} s_{1}\right)^{-1} c^{\prime} c m
$$

Claramente, $\left(s_{1}^{-1} a_{1}\right)\left(s_{2}{ }^{-1} a_{2}\right)=\left(d^{\prime} s_{1}\right)^{-1}\left(c^{\prime} d a_{2}\right)$. Notemos que $c^{\prime}\left(d a_{2}\right)=c^{\prime}(c t)$, o que nos dá $\left(d^{\prime} s_{1}\right)^{-1}\left(c^{\prime} d a_{2}\right) \cdot t^{-1} m=\left(d^{\prime} s_{1}\right)^{-1}\left(c^{\prime} c m\right)$, e temos a igualdade desejada.

(c) Leis distributivas

Já vimos que $\left(s^{-1} 1\right)\left(1^{-1} a\right)=s^{-1} a$, para todo $a \in A$ e $s \in S$. Assim, para mostrar a lei distributiva à esquerda, é suficiente mostrar que

$$
1^{-1} a \cdot\left(t^{-1} m_{1}+t^{-1} m_{2}\right)=1^{-1} a \cdot t^{-1} m_{1}+1^{-1} a \cdot t^{-1} m_{2},
$$

para todo $m_{1}, m_{2} \in M, a \in A, t \in S$.

Demonstração. Existem $v_{1} \in S, v_{2} \in A$ tais que $v_{1} a=v_{2} t$. Assim,

$$
\begin{aligned}
1^{-1} a \cdot t^{-1}\left(m_{1}+m_{2}\right) & =v_{1}^{-1}\left(v_{2}\left(m_{1}+m_{2}\right)\right)=v_{1}^{-1}\left(v_{2} m_{1}\right)+v_{1}^{-1}\left(v_{2} m_{2}\right) \\
& =1^{-1} a \cdot t^{-1} m_{1}+1^{-1} a \cdot t^{-1} m_{2} .
\end{aligned}
$$


Para a lei distributiva à direita, notemos que $t^{-1} 1 \cdot 1^{-1} m=t^{-1} m$, para todo $m \in M$, $t \in S$. Assim, utilizando a validade das leis distributivas em $S^{-1} A$, basta mostrar que

$$
\left(s^{-1} a_{1}+s^{-1} a_{2}\right) \cdot 1^{-1} m=s^{-1} a_{1} \cdot 1^{-1} m+s^{-1} a_{2} \cdot 1^{-1} m,
$$

para todo $m \in M, a_{1}, a_{2} \in A$ e $s \in S$.

Demonstração. Existem $c \in A, d \in S$ tais que $d\left(a_{1}+a_{2}\right)=c 1$. Assim,

$$
\begin{aligned}
s^{-1}\left(a_{1}+a_{2}\right) \cdot 1^{-1} m & =(d s)^{-1} c m=(d s)^{-1}\left(d\left(a_{1}+a_{2}\right)\right) m \\
& =(d s)^{-1} d\left(a_{1} m+a_{2} m\right)=s^{-1}\left(a_{1} m\right)+s^{-1}\left(a_{2} m\right) \\
& =s^{-1} a_{1} \cdot 1^{-1} m+s^{-1} a_{2} \cdot 1^{-1} m .
\end{aligned}
$$

Observação 4.2.14. Sejam $A$ um anel, $M$ um $A$-módulo à esquerda, e $S \subseteq A$ um conjunto de Ore à esquerda, formado apenas por elementos regulares de $A$. A aplicação $g: M \longrightarrow S^{-1} M$ definida por $g(m)=1^{-1} m$, para todo $m \in M$, é um homomorfismo de $A$-módulos quando $S^{-1} M$ é considerado como um $A$-módulo, usando o homomorfismo injetor de anéis $f: A \longrightarrow S^{-1} A$ (isto é, pela restrição de escalares). Notemos que $\operatorname{Ker} g=\{m \in M \mid s m=0$, para algum $s \in S\}$.

Podemos então concluir que $S^{-1} M$ satisfaz as condições da Definição 4.2.13.

Para o caso geral, em que $S$ pode eventualmente possuir divisores de zero em $A$, temos o seguinte teorema:

Teorema 4.2.15. Sejam $A$ um anel, $S \subseteq A$ um conjunto denominador à esquerda (respectivamente à direita), e $M$ um A-módulo à esquerda (respectivamente à direita). Então, existe um módulo de frações de $M$ com respeito a $S$.

(vide [10, p.173]).

Ainda de acordo com ([10, p.174]), o módulo de frações de $M$ é único, a menos de isomorfismo.

A partir daqui, estudaremos a caracterização dos módulos de frações, que são também chamamos de localização de módulos, como um funtor da categoria dos $A$-módulos na categoria $\operatorname{dos} S^{-1} A$ módulos. Faremos as construções apenas para o caso em que $S$ é um conjunto formado por elementos regulares de $A$, e assumiremos que $S$ é um conjunto de Ore à esquerda. Como usual, todas as construções e definições também podem ser efetuadas quando $S$ for um conjunto de Ore à direita.

Proposição 4.2.16. Sejam $A$ um anel, $S \subseteq A$ um conjunto de Ore à esquerda, formado apenas por elementos regulares de $A$, e $S^{-1} A$ o anel de frações à esquerda de $A$ com respeito a $S$. Para quaisquer A-módulos $M, N$ à esquerda e um homomorfismo de A-módulos à esquerda $f: M \longrightarrow N$, definimos:

$$
\begin{aligned}
S^{-1} f: S^{-1} M & \longrightarrow S^{-1} N \\
S^{-1} f\left(s^{-1} m\right) & \longmapsto s^{-1}(f(m)),
\end{aligned}
$$

para todo $m \in M$ e todo $s \in S$. $S^{-1} f$ é um homomorfismo de $S^{-1} A$-módulos. 
Demonstração. (1) Para todo $m, n \in M$ e todo $s, t \in S$, $\operatorname{com} s^{-1} m=t^{-1} n$, existem $c, d \in A$ tais que $c s=d t \in S$ e $c m=d n$. Assim,

$$
f(c m)=f(d n) \Longrightarrow c f(m)=d f(n) \Longrightarrow s^{-1}(f(m))=t^{-1}(f(n)) .
$$

Portanto, $S^{-1} f$ está bem definida.

(2) Para todo $m_{1}, m_{2} \in M$ e todo $s_{1}, s_{2} \in S$, existem $c, d \in A$ tais que $c s_{1}=d s_{2}=s \in S$. Assim,

$$
\begin{aligned}
S^{-1} f\left(s_{1}^{-1} m_{1}+s_{2}{ }^{-1} m_{2}\right) & =S^{-1} f\left(s^{-1}\left(c m_{1}+d m_{2}\right)\right)=s^{-1} f\left(c m_{1}+d m_{2}\right)=s^{-1}\left(f\left(c m_{1}\right)+f\left(d m_{2}\right)\right) \\
& =s^{-1} f\left(c m_{1}\right)+s^{-1} f\left(d m_{2}\right)=S^{-1} f\left(s^{-1}\left(c m_{1}\right)\right)+S^{-1} f\left(s^{-1}\left(d m_{2}\right)\right) \\
& =S^{-1} f\left(s_{1}^{-1} m_{1}\right)+S^{-1} f\left(s_{2}{ }^{-1} m_{2}\right),
\end{aligned}
$$

pois $S^{-1} f$ está bem definida.

Para todo $a \in A, m \in M$ e $s, t \in S$, existem $b \in A$ e $v \in S$ tais que $v a=b t$. Assim,

$$
\begin{aligned}
S^{-1} f\left(s^{-1} a \cdot t^{-1} m\right) & =S^{-1} f\left((v s)^{-1} b m\right)=(v s)^{-1} f(b m)=(v s)^{-1}(b f(m)) \\
& =s^{-1} a \cdot S^{-1} f\left(t^{-1} m\right) .
\end{aligned}
$$

Portanto, $S^{-1} f$ é um homomorfismo de $S^{-1} A$-módulos.

Proposição 4.2.17. Sejam $A$ um anel, $S \subseteq A$ um conjunto de Ore à esquerda, formado apenas por elementos regulares de $A$, e $M$ um A-módulo à esquerda. Então, a localização de $M$ com respeito a $S$ é um funtor (covariante) da categoria dos $A$-módulos à esquerda, na categoria dos $S^{-1} A$-módulos à esquerda. Denotaremos este funtor por $S^{-1}$.

Demonstração. (1) Pelo processo de construção do módulo de frações, observamos que $S^{-1}$ "leva"um objeto da categoria dos $A$-módulos à esquerda em um objeto da categoria dos $S^{-1} A$-módulos à esquerda.

(2) Pela Proposição 4.2.16, $S^{-1}$ "leva"um homomorfismo $f$ de $A$-módulos à esquerda em um homomorfismo $S^{-1} f$ de $S^{-1} A$ - módulos à esquerda.

(3) Para quaisquer $A$-módulos à esquerda $L, M, N$ e homomorfismos de $A$-módulos $f: L \longrightarrow M$ e $g: M \longrightarrow N, S^{-1}(g \circ f)=S^{-1} g \circ S^{-1} f$, o que não é difícil de notar, pela própria definição.

(4) Sejam $M, N A$-módulos à esquerda; $1_{M}: M \longrightarrow M$ o homomorfismo identidade de $M$; $f: M \longrightarrow N, g: N \longrightarrow M$ homomorfismos de $A$-módulos. Então, $S^{-1}\left(1_{M}\right)=1_{S^{-1} M}$, em que $1_{S^{-1} M}: S^{-1} M \longrightarrow S^{-1} M$ é o homomorfismo identidade de $S^{-1} M$. Notemos que, na categoria dos $S^{-1} A$-módulos, para cada um dos objetos, o homomorfismo identidade é único. Assim, basta observar que $S^{-1} f \circ S^{-1}\left(1_{M}\right)=S^{-1}\left(f \circ 1_{M}\right)=S^{-1} f$ e $S^{-1}\left(1_{M}\right) \circ S^{-1} g=$ $S^{-1}\left(1_{M} \circ g\right)=S^{-1} g$, pelo item anterior, e temos a igualdade desejada. 
Observação 4.2.18. $S^{-1}$ é um funtor aditivo, isto é, para todo $f, f^{\prime}: M \longrightarrow N$, com $M, N$ $A$-módulos à esquerda, e $f, f^{\prime}$ homomorfismos de $A$-módulos, $S^{-1}\left(f+f^{\prime}\right)=S^{-1} f+S^{-1} f^{\prime}$.

Demonstração.

$$
\begin{aligned}
S^{-1}\left(f+f^{\prime}\right)\left(s^{-1} m\right) & =s^{-1}\left(\left(f+f^{\prime}\right)(m)\right) \\
& =s^{-1}\left(f(m)+f^{\prime}(m)\right)=s^{-1} f(m)+s^{-1} f^{\prime}(m)=S^{-1} f\left(s^{-1} m\right)+S^{-1} f^{\prime}\left(s^{-1} m\right),
\end{aligned}
$$

para todo $m \in M$ e todo $s \in S$.

Proposição 4.2.19. $S^{-1}$ é um funtor exato, isto é, dada a sequência exata de A-módulos à esquerda e homomorfismos de A-módulos

$$
0 \longrightarrow L \stackrel{f}{\longrightarrow} M \stackrel{g}{\longrightarrow} N \longrightarrow 0
$$

a sequência

$$
0 \longrightarrow S^{-1} L \stackrel{S^{-1} f}{\longrightarrow} S^{-1} M \stackrel{S^{-1} g}{\longrightarrow} S^{-1} N \longrightarrow 0
$$

também é exata.

Demonstração. Como a sequência de $A$-módulos e homomorfismos de $A$-módulos é exata,

$$
\operatorname{Ker} g=\operatorname{Im} f \Longrightarrow g \circ f=0 \Longrightarrow S^{-1}(g \circ f)=0=S^{-1} g \circ S^{-1} f \Longrightarrow \operatorname{Im} S^{-1} f \subseteq \operatorname{Ker} S^{-1} g,
$$

em que 0 denota o homomorfismo nulo. Seja agora $s^{-1} m \in \operatorname{Ker} S^{-1} g$, em que $s \in S$ e $m \in M$. Então,

$$
S^{-1} g\left(s^{-1} m\right)=s^{-1} g(m)=1^{-1} 0 \Longrightarrow \text { existem } c, d \in A \text { tais que } c s=d 1 \in S \text { e } c g(m)=d 0=0 \text {, }
$$

ou seja, existe $c \in A$ tal que $g(\mathrm{~cm})=0$, e $\mathrm{cm} \in \operatorname{Ker} g$. Como a sequência original é exata, existe $l \in L$ tal que $f(l)=c m$. Assim,

$$
s^{-1} m=(c s)^{-1} c m=(c s)^{-1} f(l)=S^{-1} f\left((c s)^{-1} l\right) \in \operatorname{Im} S^{-1} f .
$$

$S^{-1} f$ é injetora. De fato, sejam $l_{1}, l_{2} \in L, s_{1}, s_{2} \in S$ tais que $S^{-1} f\left(s_{1}{ }^{-1} l_{1}\right)=S^{-1} f\left(s_{2}{ }^{-1} l_{2}\right)$; existem $c, d \in A$ tais que $c s_{1}=d s_{2} \in S$ e $c f\left(l_{1}\right)=d f\left(l_{2}\right)$; como $f$ é injetora,

$$
f\left(c l_{1}\right)=f\left(d l_{2}\right) \Longrightarrow c l_{1}=d l_{2} \Longrightarrow\left(c s_{1}\right)^{-1}\left(c l_{1}\right)=\left(d s_{2}\right)^{-1}\left(d l_{2}\right) \Longrightarrow s_{1}{ }^{-1} l_{1}=s_{2}{ }^{-1} l_{2} .
$$

$S^{-1} g$ é sobrejetora, pois para todo $n \in N$, existe $m \in M$ tal que $n=g(m)$, já que $g$ é sobrejetora. Assim, para todo $s \in S, s^{-1} n=s^{-1} g(m)=S^{-1} g\left(s^{-1} m\right)$. 
Finalizando esta subseção, estudaremos os módulos de frações sob o ponto de vista de produto tensorial.

Lema 4.2.20. Sejam $A$ um anel, $S \subseteq A$ um conjunto de Ore à esquerda, formado apenas por elementos regulares, $M$ um $A$-módulo à esquerda e $S^{-1} A$ e $S^{-1} M$ como construídos anteriormente. Definimos:

$$
\begin{aligned}
f: S^{-1} A \times M & \longrightarrow S^{-1} M \\
\left(s^{-1} a, m\right) & \longmapsto\left(s^{-1} a\right) \cdot\left(1^{-1} m\right),
\end{aligned}
$$

para todo $s \in S, a \in A$ e $m \in M$. Então, $f$ é uma função A-biaditiva.

Demonstração. (1) $f$ está bem definida:

Sejam $a_{1}, a_{2} \in A, s_{1}, s_{2} \in S$ tais que $s_{1}{ }^{-1} a_{1}=s_{2}{ }^{-1} a_{2}$. Então, existem $c, d \in A$ tais que $c s_{1}=d s_{2} \in S$ e $c a_{1}=d a_{2}$. Temos

$$
\begin{aligned}
& f\left(s_{1}{ }^{-1} a_{1}, m\right)=\left(s_{1}{ }^{-1} a_{1}\right) \cdot\left(1^{-1} m\right)=s_{1}^{-1}\left(a_{1} m\right) \mathrm{e} \\
& f\left(s_{2}{ }^{-1} a_{2}, m\right)=\left(s_{2}{ }^{-1} a_{2}\right) \cdot\left(1^{-1} m\right)=s_{2}{ }^{-1}\left(a_{2} m\right)
\end{aligned}
$$

Como $\left(c a_{1}\right) m=\left(d a_{2}\right) m$, temos o resultado desejado.

(2) $f$ é de fato $A$-biaditiva:

Para $m_{1}, m_{2} \in M, a \in A, s \in S$,

$$
\begin{aligned}
f\left(s^{-1} a, m_{1}+m_{2}\right) & =s^{-1} a \cdot\left(1^{-1}\left(m_{1}+m_{2}\right)\right)=s^{-1}\left(a\left(m_{1}+m_{2}\right)\right)=s^{-1}\left(a m_{1}\right)+s^{-1}\left(a m_{2}\right) \\
& =f\left(s^{-1} a, m_{1}\right)+f\left(s^{-1} a, m_{2}\right) .
\end{aligned}
$$

Para $a_{1}, a_{2} \in A, s_{1}, s_{2} \in S, m \in M$,

$$
\begin{aligned}
f\left(s_{1}^{-1} a_{1}+s_{2}{ }^{-1} a_{2}, m\right) & =s^{-1}\left(c a_{1}+d a_{2}\right) \cdot 1^{-1} m=s^{-1}\left(\left(c a_{1}+d a_{2}\right) m\right)=s^{-1}\left(\left(c a_{1}\right) m+\left(d a_{2}\right) m\right) \\
& =s^{-1}\left(\left(c a_{1}\right) m\right)+s^{-1}\left(\left(d a_{2}\right) m\right)=f\left(s_{1}{ }^{-1} a_{1}, m\right)+f\left(s_{2}{ }^{-1} a_{2}, m\right)
\end{aligned}
$$

com $c, d \in A$, tais que $c s_{1}=d s_{2}=s \in S$.

Para $m \in M, a, b \in A, s \in S$, podemos identificar $a \in A$ com sua imagem isomórfica $1^{-1} a \in S^{-1} A$, e temos:

$$
\begin{aligned}
f\left(\left(s^{-1} b\right) a, m\right) & =f\left(\left(s^{-1} b\right)\left(1^{-1} a\right), m\right)=f\left(s^{-1}(b a), m\right)=s^{-1}(b a) \cdot 1^{-1} m=s^{-1}((b a) m) \\
& =s^{-1}(b(a m))=f\left(s^{-1} b, a m\right) .
\end{aligned}
$$

Proposição 4.2.21. Sejam $A$ um anel, $S \subseteq A$ um conjunto de Ore à esquerda, formado apenas por elementos regulares de $A$, e $S^{-1} A, S^{-1} M$ como construídos anteriormente. Então, 


$$
S^{-1} M \cong S^{-1} A \otimes_{A} M\left(\text { como } S^{-1} A-\text { módulos }\right) .
$$

Demonstração. Pelo Lema 4.2.20, $f$ é uma função $A$-biaditiva. Logo, pela propriedade universal do produto tensorial, existe um único homomorfismo de grupos abelianos $g: S^{-1} A \otimes_{A} M \longrightarrow S^{-1} M$, tal que $g \circ \otimes=f$, isto é, $g\left(\left(s^{-1} a\right) \otimes m\right)=f\left(s^{-1} a, m\right)=s^{-1} a \cdot 1^{-1} m=s^{-1}(a m)$, para todo $a \in A$, $m \in M, s \in S$.

(a) $g$ é homomorfismo de $S^{-1} A$-módulos à esquerda:

De fato, para todo $m_{i} \in M, s, s_{i} \in S, a, a_{i} \in A, i=1, \ldots, k, k \in \mathbb{N}$ temos:

$$
\begin{aligned}
g\left(s^{-1} a\left(\sum_{i=1}^{k}\left(\left(s_{i}{ }^{-1} a_{i}\right) \otimes m_{i}\right)\right)\right) & =g\left(\sum_{i=1}^{k}\left(\left(s^{-1} a\right)\left(s_{i}^{-1} a_{i}\right) \otimes m_{i}\right)\right)=g\left(\sum_{i=1}^{k}\left(\left(s_{i}^{\prime} s\right)^{-1}\left(a_{i}^{\prime} a_{i}\right) \otimes m_{i}\right)\right) \\
& =\sum_{i=1}^{k}\left(g\left(\left(s_{i}^{\prime} s\right)^{-1}\left(a_{i}^{\prime} a_{i}\right) \otimes m_{i}\right)\right)=\sum_{i=1}^{k}\left(s_{i}^{\prime} s\right)^{-1}\left(\left(a_{i}^{\prime} a_{i}\right) m_{i}\right) \\
& =\sum_{i=1}^{k}\left(s_{i}^{\prime} s\right)^{-1}\left(\left(a_{i}^{\prime}\left(a_{i} m_{i}\right)\right)=s^{-1} a\left(g\left(\sum_{i=1}^{k}\left(\left(s_{i}^{-1} a_{i}\right) \otimes m_{i}\right)\right)\right),\right.
\end{aligned}
$$

$\operatorname{com} a_{i}^{\prime} \in A$ e $s_{i}^{\prime} \in S$ tais que $s_{i}^{\prime} a=a_{i}^{\prime} s_{i}$.

(b) $g$ é sobrejetora, pois todo elemento em $S^{-1} M$ é da forma $s^{-1} m$, para algum $m \in M$ e $s \in S$, por construção; assim, $s^{-1} m=f\left(\left(s^{-1} 1\right), m\right)=g\left(s^{-1} 1 \otimes m\right)$.

(c) $g$ é injetora

De fato, um elemento de $S^{-1} A \otimes_{A} M$ é da forma

$$
\sum_{i=1}^{k}\left(s_{i}^{-1} a_{i} \otimes m_{i}\right)
$$

com $m_{i} \in M, s_{i} \in S, a_{i} \in A, i=1, \ldots, k, k \in \mathbb{N}$. Pelo Lema A.1.4, existem $x_{1}, \ldots, x_{k} \in A$ e $y \in S$ tais que $s_{i}^{-1} a_{i}=y^{-1} x_{i}$, para todo $i=1, \ldots, k$, ou seja,

$$
\sum_{i=1}^{k}\left(s_{i}{ }^{-1} a_{i} \otimes m_{i}\right)=\sum_{i=1}^{k}\left(y^{-1} x_{i} \otimes m_{i}\right)=\sum_{i=1}^{k}\left(y^{-1} \otimes\left(x_{i} m_{i}\right)\right)=y^{-1} \otimes\left(\sum_{i=1}^{k}\left(x_{i} m_{i}\right)\right) .
$$

Assim, todo elemento em $S^{-1} A \otimes_{A} M$ é da forma $y^{-1} \otimes m$, para alguns $m \in M, y \in S$ e

$$
g\left(\sum_{i=1}^{k}\left(s_{i}^{-1} a_{i} \otimes m_{i}\right)\right)=g\left(y^{-1} \otimes m\right) .
$$

Se $g\left(y^{-1} \otimes m\right)=1^{-1} 0$, então $1^{-1} 0=y^{-1} m \Longrightarrow$ existe $c \in S$ tal que $\mathrm{cm}=0$. Assim, $y^{-1} \otimes m=y^{-1}\left(c^{-1} c\right) \otimes m=y^{-1} c^{-1} \otimes(c m)=(c y)^{-1} \otimes(c m)=0$ 
Observação 4.2.22. Se $A$ for uma álgebra sobre um corpo $\mathbb{K}$, então $S^{-1} A$ é uma álgebra sobre o mesmo corpo $\mathbb{K}$, basta definir a multiplicação por escalar da seguinte forma:

$$
\begin{aligned}
\mathbb{K} \times S^{-1} A & \longrightarrow S^{-1} A \\
\left(\alpha, s^{-1} a\right) & \longmapsto s^{-1}(\alpha a),
\end{aligned}
$$

para todo $\alpha \in \mathbb{K}, a \in A, s \in S$.

(a) $1\left(s^{-1} a\right)=s^{-1}(1 a)=s^{-1} a$, para todo $a \in A, s \in S$, com $1 \in \mathbb{K}$ o elemento identidade de $\mathbb{K}$;

(b) para todo $a_{1}, a_{2} \in A, s \in S$ e $\alpha \in \mathbb{K}$,

$$
\begin{aligned}
\alpha\left(s^{-1} a_{1}+s^{-1} a_{2}\right) & =\alpha\left(s^{-1}\left(a_{1}+a_{2}\right)\right)=s^{-1}\left(\alpha\left(a_{1}+a_{2}\right)\right)=s^{-1}\left(\alpha a_{1}+\alpha a_{2}\right) \\
& =s^{-1}\left(\alpha a_{1}\right)+s^{-1}\left(\alpha a_{2}\right)=\alpha\left(s^{-1} a_{1}\right)+\alpha\left(s^{-1} a_{2}\right) .
\end{aligned}
$$

(c) para todo $\alpha, \beta \in \mathbb{K}, a \in A, s \in S$,

$$
(\alpha+\beta) s^{-1} a=s^{-1}((\alpha+\beta) a)=s^{-1}((\alpha a)+(\beta a))=s^{-1}(\alpha a)+s^{-1}(\beta a)=\alpha\left(s^{-1} a\right)+\beta\left(s^{-1} a\right)
$$

(d) para todo $\alpha, \beta \in \mathbb{K}, a \in A, s \in S$,

$$
\alpha\left(\beta\left(s^{-1} a\right)\right)=\alpha\left(s^{-1}(\beta a)\right)=s^{-1}(\alpha(\beta a))=s^{-1}((\alpha \beta) a)=(\alpha \beta)\left(s^{-1} a\right)
$$

(e) para todo $\alpha \in \mathbb{K}, a_{1}, a_{2} \in A, s_{1}, s_{2} \in S$, existem $c \in A$ e $d \in S$ tais que $d a_{1}=c s_{2} \Longrightarrow \alpha\left(d a_{1}\right)=\alpha\left(c s_{2}\right) \Longrightarrow d\left(\alpha a_{1}\right)=(\alpha c) s_{2}$, o que nos dá

$$
\begin{aligned}
\alpha\left(\left(s_{1}{ }^{-1} a_{1}\right)\left(s_{2}{ }^{-1} a_{2}\right)\right) & =\alpha\left(\left(d s_{1}\right)^{-1}\left(c a_{2}\right)\right)=\left(d s_{1}\right)^{-1}\left(\alpha\left(c a_{2}\right)\right) \\
\left(\alpha\left(s_{1}{ }^{-1} a_{1}\right)\right) s_{2}{ }^{-1} a_{2} & =\left(s_{1}{ }^{-1}\left(\alpha a_{1}\right)\right) s_{2}{ }^{-1} a_{2}=\left(d s_{1}\right)^{-1}\left((\alpha c) a_{2}\right)=\left(d s_{1}\right)^{-1}\left(\alpha\left(c a_{2}\right)\right) \\
s_{1}{ }^{-1} a_{1}\left(\alpha\left(s_{2}{ }^{-1} a_{2}\right)\right) & =s_{1}{ }^{-1} a_{1}\left(s_{2}{ }^{-1}\left(\alpha a_{2}\right)\right)=\left(d s_{1}\right)^{-1}\left(c\left(\alpha a_{2}\right)\right)=\left(d s_{1}\right)^{-1}\left(\alpha\left(c a_{2}\right)\right) .
\end{aligned}
$$

Observação 4.2.23. Se $M$ é um $A$-módulo à esquerda sobre a álgebra $A$, então $S^{-1} M$ é um módulo à esquerda sobre a álgebra $S^{-1} A$. Basta definir a multiplicação por um escalar de $\mathbb{K}$ :

$$
\begin{aligned}
\mathbb{K} \times S^{-1} M & \longrightarrow S^{-1} M \\
\left(\alpha, s^{-1} m\right) & \longmapsto s^{-1}(\alpha m),
\end{aligned}
$$

para todo $m \in M, s \in S, \alpha \in \mathbb{K}$, e procedendo de modo análogo ao feito na Observação 4.2.22, mostra-se que $S^{-1} M$ é um espaço vetorial sobre $\mathbb{K}$ e que

$$
\alpha\left(s_{1}{ }^{-1} a \cdot s_{2}{ }^{-1} m\right)=\left(\alpha\left(s_{1}{ }^{-1} a\right)\right) \cdot s_{2}{ }^{-1} m=s_{1}{ }^{-1} a \cdot\left(\alpha\left(s_{2}{ }^{-1} m\right)\right),
$$

para todo $m \in M, s_{1}, s_{2} \in S, \alpha \in \mathbb{K}$ e $a \in A$. 


\subsection{Um Caso Particular}

Esta seção dedica-se à aplicação da teoria de localização de anéis não necessariamente comutativos (ou álgebras não necessariamente comutativas) ao caso particular estudado neste trabalho.

Em primeiro lugar, seja $\mathfrak{g}=\hat{\mathfrak{s} l}(2, \mathbb{C})$ e denotemos por $\mathfrak{U}$ a álgebra envolvente universal $\mathfrak{U}(\mathfrak{g})$. Notemos que o processo estudado na seção anterior pode ser aplicado a $\mathfrak{U}$, pois trata-se de uma álgebra associativa, com unidade, e que não possui divisores de zero (Apêndice A). Precisamos então definir o conjunto $S \subseteq \mathfrak{U}$ em relação ao qual será feita a localização.

Ainda pelo Apêndice A, recordemos que há uma "cópia"de $\mathfrak{g}$ em $\mathfrak{U}$, de onde, em particular, $e_{i}, f_{i}$ são elementos de $\mathfrak{U}$, para todo $i \in \mathbb{Z}$. Assim, para $g=e_{i}$ ou $g=f_{i}$, definimos nosso conjunto multiplicativo:

$$
S_{g}:=\left\{g^{n} \mid n \in \mathbb{Z}_{\geq 0}\right\} \subseteq \mathfrak{U},
$$

formado apenas por elementos regulares, já que $\mathfrak{U}$ não possui divisores de zero e $0 \notin S_{g}$. Notemos ainda que $1 \in S_{g}$.

Para verificar que $S_{g}$ é um conjunto de Ore, necessitamos do seguinte:

Definição 4.3.1. ([17]) Seja $\mathcal{A}$ uma álgebra associativa e seja $S$ um subconjunto multiplicativo (por convenção: $0 \notin S$ ). Um elemento $s \in S$ satisfaz as condições de localizabilidade de Ore se para qualquer $a \in \mathcal{A}$, existem $s^{\prime}, s^{\prime \prime} \in S, a^{\prime}, a^{\prime \prime} \in \mathcal{A}$ tais que $s a^{\prime}=a s^{\prime}$ e $a^{\prime \prime} s=s^{\prime \prime} a$. O conjunto $S$ satisfaz a condição de localizabilidade de Ore se todo $s \in S$ satisfaz as condições acima.

Lema 4.3.2. ([17]) Seja $\mathcal{A}$ uma álgebra associativa e seja $S$ um subconjunto multiplicativo gerado por elementos localmente ad-nilpotentes, isto é, para todo $s \in S$ tal que $s$ esteja no conjunto de geradores, $a d^{N_{a}}(s)(a)=0$, para todo $a \in \mathcal{A}$, e algum $N_{a} \in \mathbb{N}$. Então $S$ satisfaz a condição de localizabilidade de Ore.

Conforme Capítulo 1 , tanto $e_{i}$ como $f_{i}$ são elementos ad-localmente nilpotentes em $\mathfrak{g}$, para todo $i \in \mathbb{Z}$. Assim, como a representação adjunta de $\mathfrak{g}$ estende-se unicamente a uma representação de $\mathfrak{g}$ em $\mathfrak{U}$, conforme o Apêndice A, são também ad-localmente nilpotentes em $\mathfrak{U}$, e podemos usar o Lema 4.3.2 para concluir que $S_{g}$ é um conjunto de Ore.

Portanto, existe a localização de $\mathfrak{U}$ com relação a $S_{g}$, e será denotada por $\mathcal{D}_{g} \mathfrak{U}$. Temos ainda que há a imersão de $\mathfrak{U}$ em $\mathcal{D}_{g} \mathfrak{U}$, como uma subálgebra.

Para cada $\mathfrak{g}$-módulo $M$, denotaremos a localização de $M$ com relação a $S_{g}$ por $\mathcal{D}_{g} M$ e temos $\mathcal{D}_{g} M \cong \mathcal{D}_{g} \mathfrak{U} \otimes_{\mathfrak{U}} M$

Para $z \in \mathbb{C}$ e $u \in \mathcal{D}_{g} \mathfrak{U}$, definimos:

$$
\Theta_{z}(u):=\sum_{j \geq 0}\left(\begin{array}{l}
z \\
j
\end{array}\right)(a d g)^{j}(u) g^{-j}
$$

em que $\left(\begin{array}{c}z \\ j\end{array}\right)=\frac{z(z-1) \ldots(z-j+1)}{j !}$, que é uma soma finita, uma vez que $a d(g)$ é localmente nilpotente em $\mathfrak{U}$, e um elemento em $\mathcal{D}_{g} \mathfrak{U}$ escreve-se da forma $u=u^{\prime} g^{-n}$, para algum $u^{\prime} \in \mathfrak{U}$ e $n \in \mathbb{Z}_{\geq 0}$.

Em geral, para álgebras associativas, vale a seguinte fórmula de comutação à esquerda: 


$$
x^{n} y=\sum_{i=0}^{n}\left(\begin{array}{l}
n \\
i
\end{array}\right)\left(\operatorname{ad}(x)^{i} y\right) x^{n-i}
$$

para todo $x, y \in \mathcal{A}$ e $n \in \mathbb{N}$.

No nosso caso, o anel localização de $\mathcal{A}$ é uma álgebra associativa que contém uma cópia de $\mathcal{A}$. Assim, em particular, a igualdade (4.3) é válida.

Podemos então obter a seguinte relação:

$$
s^{n} y s^{-n}=\sum_{i=1}^{n}\left(\begin{array}{l}
n \\
i
\end{array}\right) a d(s)^{i} y s^{-i},
$$

para todo $s$ e $y$ no anel localização de $\mathcal{A}$, e $n \in \mathbb{N}$, concluindo que quando $z \in \mathbb{N}$,

$$
\Theta_{z}(u)=g^{z} u g^{-z} .
$$




\section{Capítulo 5}

\section{Localização da Primeira Realização de Campos Livres}

Este capítulo dedica-se ao estudo de um dos resultados desenvolvidos em [7]. Em primeiro lugar, partindo da primeira realização de campos livres de $\hat{\mathfrak{s} l}(2, \mathbb{C})$, e fixando um número inteiro, novos módulos são obtidos. Em seguida, estudamos a demonstração do teorema que fornece critérios de irredutibilidade para os mesmos.

Ao longo do texto a notação será a que já foi fixada nos capítulos anteriores. Porém, com o objetivo de simplificar a leitura, exibiremos novamente alguns conceitos e resultados importantes, além de introduzir alguma linguagem específica para o que será aqui estudado.

\subsection{Notação e Convenções}

Seja $\dot{\mathfrak{g}}=\mathfrak{s l}(2, \mathbb{C})$, e (.| .) sua forma de Killing. Consideremos a álgebra de Lie afim

$$
\mathfrak{g}=\hat{\mathfrak{s} l}(2, \mathbb{C})=\left(\mathfrak{s l}(2, \mathbb{C}) \otimes \mathbb{C}\left[t, t^{-1}\right]\right) \oplus \mathbb{C} c \oplus \mathbb{C} d,
$$

em que $c$ é o elemento central, e $d$ uma derivação. As relações de comutação em $\mathfrak{g}$ são

$$
\left[a \otimes t^{m}, b \otimes t^{n}\right]=[a, b] \otimes t^{m+n}+\delta_{m,-n} m(a \mid b) c,\left[d, a \otimes t^{m}\right]=m a \otimes t^{m},[c, \mathfrak{g}]=\{0\}
$$

em que $a, b \in \dot{\mathfrak{g}}, m, n \in \mathbb{Z}$.

Fixamos uma raiz positiva $\beta$ e uma base padrão $e, f, h$ de $\mathfrak{s l}(2, \mathbb{C})$. Então,

$$
e_{n}=e \otimes t^{n}, h_{n}=h \otimes t^{n}, f_{n}=f \otimes t^{n}, n \in \mathbb{Z},
$$

junto com $c$ e $d$, formam uma base de $\mathfrak{g}$. Além disso, $\mathfrak{h}=\mathbb{C} h_{0} \oplus \mathbb{C} c \oplus \mathbb{C} d$ é uma subálgebra de Cartan de $\mathfrak{g}$. Como usual, $\Delta$ denota o sistema de raízes de $\mathfrak{g}$ correspondente a $\mathfrak{h}$. Seja $\delta \in \mathfrak{h}^{*}$ definido por $\delta\left\lceil\mathbb{C} h_{0} \oplus \mathbb{C} c=0\right.$ e $\delta(d)=1$. Então, $\Delta=\Delta^{r e} \cup \Delta^{i m}$, em que $\Delta^{i m}=\{n \delta \mid n \in \mathbb{Z}, n \neq 0\}$ e $\Delta^{r e}=\{ \pm \beta+n \delta \mid n \in \mathbb{Z}\}$. 
Utilizaremos $\mathbf{x}$ para denotar o conjunto $\left\{x_{n} \mid n \in \mathbb{Z}\right\}$ e $\mathbf{y}$ para $\left\{y_{m} \mid m \in \mathbb{N}\right\}$. De forma mais geral, para um subconjunto $I$ de $\mathbb{Z}, \mathbf{x}_{I}:=\left\{x_{n} \mid n \in I\right\}$. Similarmente, para $S \subset \mathbb{N}$, definimos $\mathbf{y}_{S}$. Além disso, para $I \subset \mathbb{Z}$ e $S \subset \mathbb{N}$, definimos $\hat{\mathbf{x}}_{I}:=\left\{x_{n} \mid n \in \mathbb{Z}, n \notin I\right\}$ e $\hat{\mathbf{y}}_{S}:=\left\{y_{m} \mid m \in \mathbb{N}, m \notin S\right\}$. Escrevemos $\hat{\mathbf{x}}_{i}$ para $\hat{\mathbf{x}}_{\{i\}}$ e $\hat{\mathbf{y}}_{j}$ para $\hat{\mathbf{y}}_{\{j\}}$.

Sejam

$$
\mathbb{C}[\mathbf{x}, \mathbf{y}]=\mathbb{C}\left[x_{n}, y_{m} \mid n \in \mathbb{Z}, m \in \mathbb{N}\right]
$$

(polinômios em $\mathbf{x}$ e $\mathbf{y}$ ) e

$$
\mathbb{C}\left[\mathbf{x}^{ \pm 1}, \mathbf{y}^{ \pm 1}\right]=\mathbb{C}\left[x_{n}^{ \pm 1}, y_{m}^{ \pm 1} \mid n \in \mathbb{Z}, m \in \mathbb{N}\right]
$$

(polinômios de Laurent em $\mathbf{x}$ e $\mathbf{y}$ ).

Mais geralmente, escrevemos $\mathbb{C}\left[\mathbf{x}_{I}, \mathbf{x}_{I^{\prime}}^{ \pm 1}, \mathbf{y}_{S}, \mathbf{y}_{S^{\prime}}^{ \pm 1}\right]$ para o anel de polinômios em $\mathbf{x}_{I}, \mathbf{y}_{S}$ e polinômios de Laurent em $\mathbf{x}_{I^{\prime}}, \mathbf{y}_{S^{\prime}}$. Por conveniência, utilizaremos também as expressões $\hat{\mathbf{x}}_{I}^{ \pm 1}$ e $\hat{\mathbf{y}}_{S}^{ \pm 1}$ quando necessário. Por exemplo, $\mathbb{C}\left[x_{i}, \hat{\mathbf{x}}_{i}^{ \pm 1}, \mathbf{y}^{ \pm 1}\right]=\mathbb{C}\left[x_{i}, \mathbf{x}_{\mathbb{Z} \backslash\{i\}}^{ \pm 1}, \mathbf{y}^{ \pm 1}\right]$.

Denotaremos por $\mathcal{A}(\mathbf{x}, \mathbf{y})$ a álgebra dos operadores diferenciais polinomiais sobre $\mathbb{C}[\mathbf{x}, \mathbf{y}]$. Como usual, $\mathcal{A}(\mathbf{x}, \mathbf{y})$ é denominada álgebra de Weyl de $\mathbf{x}$ e $\mathbf{y}$. Escrevemos $\partial_{x_{i}}$ e $\partial_{y_{j}}$ para $\frac{\partial}{\partial_{x_{i}}}$ e $\frac{\partial}{\partial_{y_{j}}}$, respectivamente.

\subsection{Preliminares}

Um g-módulo $V$ é chamado de módulo de peso se $V=\bigoplus_{\lambda \in \mathfrak{h}^{*}} V_{\lambda}$, em que

$$
V_{\lambda}=\{v \in V \mid h v=\lambda(h) v, \forall h \in \mathfrak{h}\}
$$

O conjunto de todos os $\lambda \in \mathfrak{h}^{*}$ para os quais $V_{\lambda} \neq\{0\}$ é chamado de suporte de $V$.

\subsubsection{Módulos de Verma Imaginários}

Fixamos uma decomposição triangular $\mathfrak{g}=\mathfrak{g}_{-} \oplus \mathfrak{h} \oplus \mathfrak{g}_{+}$de $\mathfrak{g}$, em que

$$
\mathfrak{g}_{ \pm}=\left(\bigoplus_{n \in \mathbb{Z}} \mathfrak{g}_{\mp \beta+n \delta}\right) \oplus\left(\bigoplus_{m \in \mathbb{N}} \mathfrak{g}_{ \pm m \delta}\right)
$$

e dado $\lambda \in \mathfrak{h}^{*}$, construimos o módulo de Verma Imaginário $\tilde{M}(\lambda)$, de peso máximo $\lambda$, como no Capítulo 2. Naquele capítulo são também elencadas suas principais propriedades, dentre as quais destacamos um critério de irredutibilidade: $\tilde{M}(\lambda)$ é irredutível se e somente se $\lambda(c) \neq 0$.

\subsubsection{Primeira Realização de Campos Livres}

Consideremos a seguinte aplicação $\rho: \mathfrak{g} \longrightarrow \mathcal{A}(\mathbf{x}, \mathbf{y}):$ 


$$
\begin{aligned}
& f_{n} \longmapsto x_{n}, \\
& h_{n} \longmapsto-2 \sum_{m \in \mathbb{Z}} x_{m+n} \partial_{x_{m}}+\delta_{n<0} y_{-n}+\delta_{n>0} 2 n K \partial_{y_{n}}+\delta_{n, 0} J, \\
& e_{n} \longmapsto-\sum_{m, k \in \mathbb{Z}} x_{k+m+n} \partial_{x_{k}} \partial_{x_{m}}+\sum_{k>0} y_{k} \partial_{x_{-k-n}}+2 K \sum_{m>0} m \partial_{y_{m}} \partial_{x_{m-n}}+(K n+J) \partial_{x_{-n}}, \\
& d \longmapsto \sum_{i \in \mathbb{Z}} i x_{i} \partial_{x_{i}}-\sum_{j>0} j y_{j} \partial_{y_{j}}, c \longmapsto K .
\end{aligned}
$$

Recordando o Capítulo 3, existe uma realização de $\mathfrak{g}$ no espaço $\mathbb{C}\left[x_{m}, m \in \mathbb{Z}\right] \otimes \mathbb{C}\left[y_{n}, n>0\right]$, em que a ação de $f_{n}, h_{n}, e_{n}, n \in \mathbb{Z}$ é a mesma de (5.1). Assim, para mostrar que $\rho$ fornece uma representação de $\mathfrak{g}$ em $\mathbb{C}[\mathbf{x}, \mathbf{y}]$, precisamos apenas mostrar que, para todo $n \in \mathbb{Z}$,

$$
\rho\left(\left[d, g_{n}\right]\right)=\left[\rho(d), \rho\left(g_{n}\right)\right] \text {, em que } g_{n}=e_{n}, f_{n}, h_{n}, n \in \mathbb{Z},
$$

o que faremos em seguida. 
Demonstração.

$$
\begin{aligned}
& {\left[\rho(d), \rho\left(f_{n}\right)\right]=\left[\sum_{i \in \mathbb{Z}} i x_{i} \partial_{x_{i}}-\sum_{j>0} j y_{j} \partial_{y_{j}}, x_{n}\right]=\left[\sum_{i \in \mathbb{Z}} i x_{i} \partial_{x_{i}}, x_{n}\right]=n x_{n}=\rho\left(n f_{n}\right)=\rho\left(\left[d, f_{n}\right]\right) ;} \\
& {\left[\rho(d), \rho\left(h_{n}\right)\right]=\left[\sum_{i \in \mathbb{Z}} i x_{i} \partial_{x_{i}}-\sum_{j>0} j y_{j} \partial_{y_{j}},-2 \sum_{m \in \mathbb{Z}} x_{m+n} \partial_{x_{m}}+\delta_{n<0} y_{-n}+\delta_{n>0} 2 n K \partial_{y_{n}}+\delta_{n, 0} J\right]} \\
& =-2\left[\sum_{i \in \mathbb{Z}} i x_{i} \partial_{x_{i}}, \sum_{m \in \mathbb{Z}} x_{m+n} \partial_{x_{m}}\right]-\left[\sum_{j>0} j y_{j} \partial_{y_{j}}, \delta_{n<0} y_{-n}\right]-\left[\sum_{j>0} j y_{j} \partial_{y_{j}}, \delta_{n>0} 2 n K \partial_{y_{n}}\right] \\
& =-2 \sum_{m \in \mathbb{Z}}(m+n) x_{m+n} \partial_{x_{m}}+2 \sum_{m \in \mathbb{Z}} x_{m+n} m \partial_{x_{m}}-(-n) y_{-n} \delta_{n<0}+\delta_{n>0} 2 n K n \partial_{y_{n}} \\
& =-2 n \sum_{m \in \mathbb{Z}} x_{m+n} \partial_{x_{m}}+n \delta_{n<0} y_{-n}+n \delta_{n>0} 2 n K \partial_{y_{n}}={ }^{(\star)} \rho\left(n h_{n}\right)=\rho\left(\left[d, h_{n}\right]\right) ; \\
& {\left[\rho(d), \rho\left(e_{n}\right)\right]=\left[\sum_{i \in \mathbb{Z}} i x_{i} \partial_{x_{i}}-\sum_{j>0} j y_{j} \partial_{y_{j}},\right.} \\
& \left.-\sum_{m, k \in \mathbb{Z}} x_{k+m+n} \partial_{x_{k}} \partial_{x_{m}}+\sum_{k>0} y_{k} \partial_{x_{-k-n}}+2 K \sum_{m>0} m \partial_{y_{m}} \partial_{x_{m-n}}+(K n+J) \partial_{x_{-n}}\right] \\
& =-\left[\sum_{i \in \mathbb{Z}} i x_{i} \partial_{x_{i}}, \sum_{m, k \in \mathbb{Z}} x_{k+m+n} \partial_{x_{k}} \partial_{x_{m}}\right]+\left[\sum_{i \in \mathbb{Z}} i x_{i} \partial_{x_{i}}, \sum_{k>0} y_{k} \partial_{x_{-k-n}}\right] \\
& +2 K\left[\sum_{i \in \mathbb{Z}} i x_{i} \partial_{x_{i}}, \sum_{m>0} m \partial_{y_{m}} \partial_{x_{m-n}}\right]+\left[\sum_{i \in \mathbb{Z}} i x_{i} \partial_{x_{i}},(K n+J) \partial_{x_{-n}}\right] \\
& -\left[\sum_{j>0} j y_{j} \partial_{y_{j}}, \sum_{k>0} y_{k} \partial_{x_{-k-n}}\right]-2 K\left[\sum_{j>0} j y_{j} \partial_{y_{j}}, \sum_{m>0} m \partial_{y_{m}} \partial_{x_{m-n}}\right] \\
& =-\sum_{m, k \in \mathbb{Z}}(k+m+n) x_{k+m+n} \partial_{x_{k}} \partial_{x_{m}}+\sum_{m, k \in \mathbb{Z}} x_{k+m+n} \partial_{x_{k}} m \partial_{x_{m}}+\sum_{m, k \in \mathbb{Z}} x_{k+m+n} \partial_{x_{m}} k \partial_{x_{k}} \\
& +\sum_{k>0} y_{k}(k+n) \partial_{x_{-k-n}}-\sum_{k>0} k y_{k} \partial_{x_{-k-n}}-2 K \sum_{m>0} m \partial_{y_{m}}(m-n) \partial_{x_{m-n}} \\
& +2 K \sum_{m>0} m \partial_{x_{m-n}} m \partial_{y_{m}}+(K n+J) n \partial_{x_{-n}} \\
& =-n \sum_{m, k \in \mathbb{Z}} x_{k+m+n} \partial_{x_{k}} \partial_{x_{m}}+n \sum_{k>0} y_{k} \partial_{x_{-k-n}}+n 2 K \sum_{m>0} m \partial_{y_{m}} \partial_{x_{m-n}}+n(K n+J) \partial_{x_{-n}} \\
& =\rho\left(n e_{n}\right)=\rho\left(\left[d, e_{n}\right]\right) .
\end{aligned}
$$

( $\star$ :a parcela $\delta_{n, 0} J$ só é não nula se $n=0$; porém, neste caso, $n \delta_{n, 0} J=0 \delta_{n, 0} J=0$.

Portanto, $\rho$ é um homomorfismo de álgebras de Lie. Sendo assim, dá origem a um homomorfismo $\Phi: \mathfrak{U}(\mathfrak{g}) \longrightarrow \mathcal{A}(\mathbf{x}, \mathbf{y})$ de álgebras associativas (Apêndice A), que será chamado de primeira realização de campos livres de $\mathfrak{g}$ ou, abreviadamente, FFFR. Com o auxílio desse homomorfismo, dotamos qualquer $\mathcal{A}(\mathbf{x}, \mathbf{y})$-módulo $M$ com uma estrutura de $\mathfrak{g}$-módulo.

Para o $\mathfrak{g}$-módulo $\mathbb{C}[\mathbf{x}, \mathbf{y}]$, em particular, obtemos uma realização dos módulos de Verma imaginários. Tal resultado foi obtido, quando $K=0$, por Jakobsen e Kac em [12]; para um $K$ arbitrário, por Bernard e Felder em [3].

Teorema 5.2.1. ([12],[3]) O $\mathfrak{g}$-módulo $\mathbb{C}[\mathbf{x}, \mathbf{y}]$ é isomorfo ao módulo de Verma Imaginário $\tilde{M}(\lambda)$, 
em que $\lambda \in \mathfrak{h}^{*}$ é definido por $\lambda\left(h_{0}\right)=J, \lambda(c)=K$ e $\lambda(d)=0$.

Observação 5.2.2. (a) Quando $\lambda(d)=D \neq 0$, ainda podemos obter uma realização de $\tilde{M}(\lambda)$, deslocando $\rho(d)$ :

$$
d \longmapsto D+\sum_{i \in \mathbb{Z}} i x_{i} \partial_{x_{i}}-\sum_{j>0} j y_{j} \partial_{y_{j}}
$$

(b) Em [12], para $K=0$, a realização é isomorfa ao quociente de $\tilde{M}(\lambda)$ pelo submódulo gerado por $h_{n} \otimes 1, n<0$. Além disso, a realização em [3] é equivalente à FFFR após a aplicação de duas anti-involuções.

De acordo com [8] e [9], se $K=0$, o quociente de $\mathbb{C}[\mathbf{x}, \mathbf{y}]$ pelo módulo gerado por $y_{m}, m>0$, é irredutível se e somente se $J \neq 0$.

Esse quociente é isomorfo a $\mathbb{C}[\mathbf{x}]$ e tem uma estrutura de $\mathfrak{g}$-módulo através do homomorfismo $\rho^{\prime}: \mathfrak{g} \longrightarrow \mathcal{A}(\mathbf{x})$ definido por

$$
\begin{aligned}
f_{n} \longmapsto x_{n} ; \\
h_{n} \longmapsto-2 \sum_{m \in \mathbb{Z}} x_{m+n} \partial_{x_{m}}+\delta_{n, 0} J ; \\
e_{n} \longmapsto-\sum_{m, k \in \mathbb{Z}} x_{k+m+n} \partial_{x_{k}} \partial_{x_{m}}+J \partial_{x_{-n}} ; \\
d \longmapsto \sum_{i \in \mathbb{Z}} i x_{i} \partial_{x_{i}}, c \longmapsto 0,
\end{aligned}
$$

que fornece também um homomorfismo de álgebras associativas $\Phi^{\prime}: \mathfrak{U}(\mathfrak{g}) \longrightarrow \mathcal{A}(\mathbf{x})$.

Para esse novo módulo, temos um resultado análogo ao Teorema 5.2.1, como segue:

Teorema 5.2.3. O $\mathfrak{g}$-módulo $\mathbb{C}[\mathbf{x}]$ é isomorfo a $\hat{M}(\lambda)$, em que $\lambda \in \mathfrak{h}^{*}$ é definido por $\lambda\left(h_{0}\right)=J$, $\lambda(c)=0$ e $\lambda(d)=0$.

$O \mathfrak{g}$-módulo $\mathbb{C}[\mathbf{x}]$ é irredutivel se e somente se $J \neq 0$. Se $J=0$, então o módulo $\mathbb{C}[\mathbf{x}]$ possui um unico quociente irredutivel isomorfo a $\mathbb{C}$.

\subsection{Irredutibilidade de Módulos Localizados}

A partir de agora, fixamos $i \in \mathbb{Z}$.

Salvo menção em contrário, cada $\mathcal{A}(\mathbf{x}, \mathbf{y})$-módulo $M$ será também considerado como um módulo sobre $\mathfrak{g}$ via $\Phi$. Por simplicidade, muitas vezes denotaremos tanto o $\mathcal{A}(\mathbf{x}, \mathbf{y})$-módulo como o correspondente $\mathfrak{g}$-módulo por $M$.

Fixando também um subconjunto finito $S=\left\{k_{1}, \ldots, k_{n}\right\}$ de $\mathbb{N}$, em que $S=\emptyset$ é admitido, definimos quatro espaços vetoriais de polinômios de Laurent:

$N_{S}:=\mathbb{C}\left[\mathbf{x}, \mathbf{y}_{S}^{ \pm 1}, \hat{\mathbf{y}}_{S}\right]$

$N_{i, S}:=\mathbb{C}\left[x_{i}^{ \pm 1}, \mathbf{y}_{S}^{ \pm 1}, \hat{\mathbf{x}}_{i}, \hat{\mathbf{y}}_{S}\right]$,

$I_{S}:=\mathbb{C}\left[\mathbf{x}, \mathbf{y}_{S \backslash\left\{k_{1}\right\}}^{ \pm 1}, \hat{\mathbf{y}}_{S \backslash\left\{k_{1}\right\}}\right]+\ldots+\mathbb{C}\left[\mathbf{x}, \mathbf{y}_{S \backslash\left\{k_{n}\right\}}^{ \pm 1}, \hat{\mathbf{y}}_{S \backslash\left\{k_{n}\right\}}\right]$, 
$I_{i, S}:=\mathbb{C}\left[\mathbf{x}, \mathbf{y}_{S}^{ \pm 1}, \hat{\mathbf{y}}_{S}\right]+\mathbb{C}\left[x_{i}^{ \pm 1}, \mathbf{y}_{S \backslash\left\{k_{1}\right\}}^{ \pm 1}, \hat{\mathbf{x}}_{i}, \hat{\mathbf{y}}_{S \backslash\left\{k_{1}\right\}}\right]+\ldots+\mathbb{C}\left[x_{i}^{ \pm 1}, \mathbf{y}_{S \backslash\left\{k_{n}\right\}}^{ \pm 1}, \hat{\mathbf{x}}_{i}, \hat{\mathbf{y}}_{S \backslash\left\{k_{n}\right\}}\right]$

Além disso, definimos:

$$
M_{S}:=N_{S} / I_{S}, M_{i, S}:=N_{i, S} / I_{i, S}
$$

Observemos que os espaços que acabamos de definir são $\mathcal{A}(\mathbf{x}, \mathbf{y})$-módulos. Assim, usando o homomorfismo de álgebras associativas $\Phi: \mathfrak{U}(\mathfrak{g}) \longrightarrow \mathcal{A}(\mathbf{x}, \mathbf{y})$, temos o seguinte resultado:

Proposição 5.3.1. O homomorfismo $\Phi$ define uma representação de $\mathfrak{g}$ nos espaços $N_{S}, N_{i, S}, M_{S}$ e $M_{i, S}$. Temos ainda os seguintes isomorfismos de espaços vetoriais:

$$
M_{S} \cong y_{k_{1}}^{-1} \ldots y_{k_{n}}^{-1} \mathbb{C}\left[\mathbf{x}, \mathbf{y}_{S}^{-1}, \hat{\mathbf{y}}_{S}\right], M_{i, S} \cong x_{i}^{-1} y_{k_{1}}^{-1} \ldots y_{k_{n}}^{-1} \mathbb{C}\left[x_{i}^{-1}, \mathbf{y}_{S}^{-1}, \hat{\mathbf{x}}_{i}, \hat{\mathbf{y}}_{S}\right]
$$

Com o auxílio dos isomorfismos dados pela Proposição 5.3.1, apresentaremos cada elemento de $M_{S}$ (respectivamente, $M_{i, S}$ ) unicamente como um polinômio com potências negativas de $y_{k_{1}}, \ldots, y_{k_{n}}$ (respectivamente, $y_{k_{1}}, \ldots, y_{k_{n}}$ e $x_{i}$ ).

Recordemos que se $K=0$ e $J \neq 0$, então o módulo $\mathbb{C}[\mathbf{x}, \mathbf{y}]$ é redutível e possui um único quociente irredutível isomorfo a $\mathbb{C}[\mathbf{x}]$.

No caso em que $K=0$, definimos:

$N_{i}:=\mathbb{C}\left[x_{i}^{ \pm 1}, \hat{\mathbf{x}}_{i}\right]$,

$I_{i}:=\mathbb{C}[\mathbf{x}]$,

$M_{i}:=N_{i} / I_{i}$.

Como os espaços definidos acima podem ser vistos como $\mathcal{A}(\mathbf{x})$-módulos, temos o seguinte:

Proposição 5.3.2. O homomorfismo $\Phi^{\prime}$ define uma representação de $\mathfrak{g}$ nos espaços vetoriais $N_{i}$ e $M_{i}$. Temos também o seguinte isomorfismo de espaços vetoriais:

$$
M_{i} \cong x_{i}^{-1} \mathbb{C}\left[x_{i}^{-1}, \hat{\mathbf{x}}_{i}\right]
$$

Com o auxílio do isomorfismo na Proposição 5.3.2, apresentaremos cada elemento de $M_{i}$ como um polinômio com potências negativas de $x_{i}$.

Com as definições e a notação estabelecidas até aqui, estudaremos os critérios de irredutibilidade dos g-módulos $M_{S}, M_{i, S}$ e $M_{i}$, que serão também denotados por $\Phi(S), \Phi(i, S)$ e $\Phi^{\prime}(i)$, respectivamente. O objetivo é a demonstração do seguinte teorema:

Teorema 5.3.3. Sejam i e $S$ como acima.

(a) Seja $K \neq 0$. Então, a representação $\Phi(S)$ é irredutivel, enquanto $\Phi(i, S)$ é irredutível se e somente se $J-i K \notin \mathbb{Z}$;

(b) Seja $K=0$. Então a representação $\Phi^{\prime}(i)$ é irredutivel se e somente se $J \notin \mathbb{Z}$.

O Teorema 5.3.3 permite-nos construir explicitamente novas representações irredutíveis de $\mathfrak{g}$, a partir de módulos de Verma imaginários. 


\subsection{Demonstração do Teorema 5.3.3}

Seja $\mathfrak{U}=\mathfrak{U}(\mathfrak{g})$ a álgebra envolvente universal de $\mathfrak{g}$. Para todo $u \in \mathfrak{U}$, e $v \in M_{S}$ ou $v \in M_{i, S}$, denotaremos $\Phi(u)(v)$ apenas por $u(v)$. De modo análogo, faremos $u^{\prime}\left(v^{\prime}\right)$ ao invés de $\Phi^{\prime}\left(u^{\prime}\right)\left(v^{\prime}\right)$, para $u^{\prime} \in \mathfrak{U}$ e $v^{\prime} \in M_{i}$. Denotar a ação de $\mathfrak{U}$ dessa forma é um abuso de notação, mas tem o objetivo de facilitar a leitura.

Em primeiro lugar, será introduzida uma notação padrão necessária para o desenvolvimento da demonstração:

Definição 5.4.1. Seja

$$
v=x_{i}^{-p} y_{k_{1}}^{-\gamma_{1}} \ldots y_{k_{n}}^{-\gamma_{n}} y_{j_{1}}^{\beta_{1}} \ldots y_{j_{l}}^{\beta_{l}} x_{i_{1}}^{\alpha_{1}} \ldots x_{i_{k}}^{\alpha_{k}} \in M_{i, S}
$$

em que $p, \gamma_{1}, \ldots, \gamma_{n}, \beta_{1}, \ldots, \beta_{l}, \alpha_{1}, \ldots, \alpha_{k} \in \mathbb{N}$. Definimos

$$
\begin{aligned}
\operatorname{deg}_{x}(v) & =-p+\alpha_{1}+\ldots+\alpha_{k}, \\
\operatorname{deg}_{x^{+}}(v) & =\alpha_{1}+\ldots+\alpha_{k}, \\
\operatorname{deg}_{y}(v) & =-\gamma_{1}-\ldots-\gamma_{n}+\beta_{1}+\ldots+\beta_{l}, \\
\operatorname{deg}_{y^{+}}(v) & =\beta_{1}+\ldots+\beta_{l} .
\end{aligned}
$$

As definições acima estendem-se naturalmente ao caso de um elemento arbitrário $v \in M_{i, S}$. Por exemplo, se $v=v_{1}+\ldots+v_{t}$, em que $v_{1}, \ldots, v_{t}$ são monômios, então definimos:

$$
\operatorname{deg}_{y}(v)=\max \left\{\operatorname{deg}_{y}\left(v_{j}\right) \mid j=1, \ldots, t\right\}
$$

e diremos que $\operatorname{deg}_{y}(v)$ é o $y$-grau de $v$. Diremos que $v=v_{1}+\ldots+v_{t}$ é $d e g_{y}$-homogêneo se os monômios $v_{1}, \ldots, v_{t}$ têm o mesmo $y$-grau. Similarmente, introduzimos $\operatorname{deg}_{x}(v)$ e $\operatorname{deg}_{x^{+}}(v)$ para um elemento arbitrário $v \in M_{i, S}$, bem como as noções de $x$-grau, $x^{+}$-grau, $d e g_{x}$-homogêneo e $d e g_{x^{+}}$-homogêneo. Finalmente, de modo análogo, introduzimos as mesmas notação e noções para os módulos $M_{S}$ e $M_{i}$, respectivamente.

\subsubsection{Vetores Primitivos no Caso $J-i K \in \mathbb{Z}$.}

Nesta subseção, focaremos no caso em que $J-i K$ é um número inteiro. O principal objetivo é provar que os módulos $M_{i, S}$ e $M_{i}$ possuem vetores $e_{-i}$-primitivos, ou seja, vetores $v$ não nulos tais que $e_{-i}(v)=0$. Como um corolário, será mostrado que $M_{i, S}$ e $M_{i}$ são redutíveis.

Após a demonstração, teremos condições de concluir o seguinte: para $K \neq 0$, se $M_{i, S}$ é irredutível, então $J-i K$ não pode ser um número inteiro; para $K=0$, se $M_{i}$ é irredutível, então $J$ não pode ser um número inteiro.

Destacamos ainda que, nesta subseção, será usada a seguinte notação:

$$
\varepsilon_{i}=\sum_{s_{1}>i, s_{2}>i} x_{s_{1}+s_{2}-i} \partial_{x_{s_{1}}} \partial_{x_{s_{2}}}
$$


Como $s_{1}>i$ e $s_{2}>i$, existem $j, k \in \mathbb{Z}$ tais que $s_{1}-i=j>0$ e $s_{2}-i=k>0$. Assim, $s_{1}+s_{2}-i=j+i+k+i-i=j+k+i$, o que nos fornece a igualdade

$$
\varepsilon_{i}=\sum_{s_{1}>i, s_{2}>i} x_{s_{1}+s_{2}-i} \partial_{x_{s_{1}}} \partial_{x_{s_{2}}}=\sum_{s_{1}, s_{2}>0} x_{s_{1}+s_{2}+i} \partial_{x_{s_{1}+i}} \partial_{x_{s_{2}+i}} .
$$

Lema 5.4.2. Seja $N>0$. Então, existe um elemento $g \in \mathbb{C}[\mathbf{x}]$, deg $g_{x}$-homogêneo, que possui grau $\operatorname{deg}(g)=\operatorname{deg}_{x}(g)=N+1$, tal que $\partial_{x_{j}}(g)=0$, para $j \leq i$ e $\varepsilon_{i}^{N}(g)=0$.

Demonstração. Sejam $i_{1}, \ldots, i_{N+1} \in \mathbb{Z}$ tais que $i_{m}>i$, para $m \in\{1, \ldots, N+1\}$. Usando indução em $N$, é possível mostrar que

$$
\varepsilon_{i}^{N}\left(x_{i_{1}} \ldots x_{i_{N+1}}\right)=c_{N} x_{M},
$$

em que $c_{N}=2^{N} \prod_{s=2}^{N+1}\left(\begin{array}{c}s \\ 2\end{array}\right)$ e $M=i_{1}+\ldots+i_{N+1}-N i$. Consequentemente, $g=x_{i_{1}} \ldots x_{i_{N+1}}-x_{i_{1}^{\prime}} \ldots x_{i_{N+1}^{\prime}}$, em que $i_{m}^{\prime}>i$, para $m \in\{1, \ldots, N+1\}$, e $\sum_{m=1}^{N+1} i_{s}=\sum_{m=1}^{N+1} i_{s}^{\prime}$, irá satisfazer as condições do lema.

Proposição 5.4.3. Seja $J-i K \in \mathbb{Z}$.

(a) Se $K \neq 0$, então existe um elemento não nulo $v \in M_{i, S}$ tal que $e_{-i}(v)=0$;

(b) Se $K=0$, então existe um elemento não nulo $v \in M_{i}$ tal que $e_{-i}(v)=0$.

Demonstração. Em primeiro lugar, para cada $K$, encontraremos um vetor $e_{-i}$-primitivo $v \in M_{i, \emptyset}$ (isto é, $S=\emptyset$ ) com a propriedade $\partial_{y_{j}}(v)=0$, para cada $j>0$. Isso, em particular, implica $(b)$.

Para esta primeira etapa, fixamos um número inteiro positivo $N$ tal que $N \geq J-i K-2$ (notemos que, como $J-i K \in \mathbb{Z}$, isso pode ser feito). A estratégia da demonstração consiste em encontrar dois vetores $e_{-i}$-primitivos no módulo $N_{i, \emptyset}$, o segundo deles não nulo em $M_{i, \emptyset}$.

Seja então $A_{t}=t(t-2 N+J-i K-3)$. Usando o Lema 5.4.2, escolhemos um polinômio homogêneo $g_{0}$ em $\mathbb{C}[\mathbf{x}]$, de grau $N+2$, com a propriedade $\partial_{x_{j^{\prime}}}=0$, para cada $j^{\prime} \leq i$, tal que $\varepsilon_{i}^{N+1}\left(g_{0}\right)=0$. Então, para $t=1, \ldots, N$, definimos $g_{t}=\frac{1}{A_{1} \ldots A_{t}} \varepsilon_{i}^{t}\left(g_{0}\right)$.

Observação 5.4.4. Notemos que, devido à escolha de $N, A_{t} \neq 0$, para $t=1, \ldots, N$. De fato, se $A_{t}=0$, então $t=0$ ou $t-2 N+J-i K-3=0$. Como $1 \leq t \leq N$, deveríamos necessariamente ter $t-2 N+J-i K-3=0$, o que forneceria $2 N-t=J-i K-3$. Mostraremos que $2 N-t>J-i K-3$.

Como $N \geq J-i K-2$, então $N>J-i K-3$. Por outro lado, como $1 \leq t \leq N$, então $2 N-t \geq N$. Assim, $2 N-t \geq N>J-i K-3$, e $A_{t} \neq 0$.

Assim, usando que $\varepsilon_{i}\left(g_{t}\right)=A_{t+1} g_{t+1}, t \geq 0$, e $\varepsilon_{i}^{N+1}\left(g_{0}\right)=0$, verificaremos que

$$
e_{-i}\left(\sum_{t=0}^{N} x_{i}^{t} g_{t}\right)=\varepsilon_{i}\left(g_{0}\right)+\sum_{t=1}^{N}\left(-A_{t} x_{i}^{t-1} g_{t}+x_{i}^{t} \varepsilon_{i}\left(g_{t}\right)\right)=0 .
$$

Demonstração. Como $\sum_{t=0}^{N} x_{i}^{t} g_{t}=g_{0}+\sum_{t=1}^{N} x_{i}^{t} g_{t}$, vamos avaliar $e_{-i}\left(g_{0}\right)+e_{-i}\left(\sum_{t=1}^{N} x_{i}^{t} g_{t}\right)$. 
Tendo em vista que $\partial_{x_{j^{\prime}}}\left(g_{0}\right)=0$, para todo $j^{\prime} \leq i$, então $e_{-i}\left(g_{0}\right)=-\varepsilon_{i}\left(g_{0}\right)$. Na sequência, vamos analisar

$$
e_{-i}\left(\sum_{t=1}^{N} x_{i}^{t} g_{t}\right)=-\sum_{m, k \in \mathbb{Z}} x_{k+m-i} \partial_{x_{k}} \partial_{x_{m}}\left(\sum_{t=1}^{N} x_{i}^{t} g_{t}\right)+(J-i K) \partial_{x_{i}}\left(\sum_{t=1}^{N} x_{i}^{t} g_{t}\right)
$$

Por um lado,

$$
(J-i K) \partial_{x_{i}}\left(\sum_{t=1}^{N} x_{i}^{t} g_{t}\right)=\sum_{t=1}^{N}(J-i K) t x_{i}^{t-1} g_{t}
$$

A outra parcela desmembra-se da seguinte forma:

$$
\begin{array}{r}
-x_{i} \partial_{x_{i}} \partial_{x_{i}}\left(\sum_{t=1}^{N} x_{i}^{t} g_{t}\right)-2 \sum_{s \in \mathbb{Z} \backslash\{i\}} x_{s} \partial_{x_{s}} \partial_{x_{i}}\left(\sum_{t=1}^{N} x_{i}^{t} g_{t}\right)-\varepsilon_{i}\left(\sum_{t=1}^{N} x_{i}^{t} g_{t}\right) \\
=-\sum_{t=1}^{N} t(t-1) x_{i} x_{i}^{t-2} g_{t}-2 \sum_{t=1}^{N}\left(\sum_{s \in \mathbb{Z} \backslash\{i\}} x_{s} \partial_{x_{s}}\left(t x_{i}^{t-1} g_{t}\right)\right)-\sum_{t=1}^{N} x_{i}^{t} \varepsilon_{i}\left(g_{t}\right) \\
=-\sum_{t=1}^{N} t(t-1) x_{i}^{t-1} g_{t}-\sum_{t=1}^{N} x_{i}^{t} \varepsilon_{i}\left(g_{t}\right)-\sum_{t=1}^{N}(2 N+4-2 t) t x_{i}^{t-1} g_{t} \\
=-\sum_{t=1}^{N} t(t-1+2 N+4-2 t) x_{i}^{t-1} g_{t}-\sum_{t=1}^{N} x_{i}^{t} \varepsilon_{i}\left(g_{t}\right) .
\end{array}
$$

Assim, $e_{-i}\left(\sum_{t=1}^{N} x_{i}^{t} g_{t}\right)=\sum_{t=1}^{N}(\underbrace{(\underbrace{(J-i K-2 N+t-3)}}_{A_{t}} x_{i}^{t-1} g_{t}-x_{i}^{t} \varepsilon_{i}\left(g_{t}\right))$, por (5.2) e (5.3), o que implica

$$
e_{-i}\left(\sum_{t=0}^{N} x_{i}^{t} g_{t}\right)=-\varepsilon_{i}\left(g_{0}\right)+\sum_{t=1}^{N}\left(A_{t} x_{i}^{t-1} g_{t}-x_{i}^{t} \varepsilon_{i}\left(g_{t}\right)\right)
$$

Usando que

$$
\begin{aligned}
& \varepsilon_{i}\left(g_{t}\right)=A_{t+1} g_{t+1}, t \geq 0, \text { temos: } \\
& e_{-i}\left(\sum_{t=0}^{N} x_{i}^{t} g_{t}\right)=-A_{1} g_{1}+\sum_{t=1}^{N}\left(A_{t} x_{i}^{t-1} g_{t}-x_{i}^{t} A_{t+1} g_{t+1}\right) .
\end{aligned}
$$

Notemos agora que as parcelas do lado direito de (5.4) cancelam-se duas a duas, "sobrando"apenas $-x_{i}^{N} A_{N+1} g_{N+1}$. Mas 


$$
A_{N+1} g_{N+1}=\varepsilon_{i}\left(g_{N}\right)=\varepsilon_{i}\left(\frac{1}{A_{1} \ldots A_{N}} \varepsilon_{i}^{N}\left(g_{0}\right)\right)=\frac{1}{A_{1} \ldots A_{N}} \underbrace{\varepsilon_{i}^{N+1}\left(g_{0}\right)}_{0}=0
$$

e temos a igualdade.

Ainda não temos o resultado desejado, pois $\sum_{t=0}^{N} x_{i}^{t} g_{t}$ é zero tanto em $M_{i, \emptyset}$, como em $M_{i}$, e ainda precisamos encontrar um vetor $e_{-i}$-primitivo. Para tanto, faz-se necessário multiplicar $\sum_{t=0}^{N} x_{i}^{t} g_{t}$ por uma potência negativa de $x_{i}$, em que o expoente seja suficientemente grande. Para atingir esse objetivo, utilizaremos a localização de $\mathfrak{U}$ e de $N_{\emptyset}$ (com relação ao conjunto $S_{f_{i}}$ ) como ferramentas, aplicando a fórmula (4.2) em $w=\sum_{t=0}^{N} x_{i}^{t} g_{t}$, para $u=e_{-i}$ e $g=f_{i}$, e o número inteiro positivo $y=2 N+3-(J-i K) \geq 1$. Explicitamente,

$$
f_{i}^{y} e_{-i} f_{i}^{-y}(w)=\sum_{j \geq 0}\left(\begin{array}{c}
y \\
j
\end{array}\right)\left(a d\left(f_{i}\right)\right)^{j}\left(e_{-i}\right) f_{i}^{-j}(w) .
$$

Cabe aqui observarmos que aplicar a fórmula (4.2) a $w \in N_{i, \emptyset}$ faz sentido apenas porque a localização do módulo $N_{\emptyset}$ com respeito a $S_{f_{i}}$ é o módulo $N_{i, \emptyset}$. Para tanto, basta recordarmos a construção explícita dos módulos de frações efetuada no Capítulo 4.

Assim, temos:

$$
\begin{aligned}
f_{i}^{y} e_{-i} f_{i}^{-y}(w) & =\left(e_{-i}+\left(\begin{array}{c}
y \\
1
\end{array}\right)\left[f_{i}, e_{-i}\right] f_{i}^{-1}+\left(\begin{array}{c}
y \\
2
\end{array}\right)\left[f_{i},\left[f_{i}, e_{-i}\right]\right] f_{i}^{-2}\right)(w) \\
& =\left(e_{-i}-\left(\begin{array}{c}
y \\
1
\end{array}\right)\left(h_{0}-i K\right) f_{i}^{-1}-2\left(\begin{array}{c}
y \\
2
\end{array}\right) f_{i}^{-1}\right)(w) .
\end{aligned}
$$

Já mostramos que $e_{-i}(w)=0$. Vamos agora avaliar $\left(-\left(\begin{array}{l}y \\ 1\end{array}\right)\left(h_{0}-i K\right) f_{i}^{-1}-2\left(\begin{array}{c}y \\ 2\end{array}\right) f_{i}^{-1}\right)(w)$ :

$\left(-\left(\begin{array}{c}y \\ 1\end{array}\right)\left(h_{0}-i K\right) f_{i}^{-1}-2\left(\begin{array}{c}y \\ 2\end{array}\right) f_{i}^{-1}\right)(w)=-y\left(h_{0}-i K+y-1\right) f_{i}^{-1}(w)$

$=-y\left(h_{0}-i K+y-1\right)\left(x_{i}^{-1} w\right)=-y\left(h_{0}\left(x_{i}^{-1} w\right)+(-i K+y-1) x_{i}^{-1} w\right)$

$=-y\left(\left(-2 \sum_{m \in \mathbb{Z}} x_{m} \partial_{x_{m}}+J\right)\left(x_{i}^{-1} w\right)+(-i K+2 N+3-(J-i K)-1) x_{i}^{-1} w\right)$

$=-y\left(-2 \sum_{t=0}^{N}\left(\sum_{m \in \mathbb{Z}} x_{m} \partial_{x_{m}}\left(x_{i}^{t-1} g_{t}\right)\right)+J x_{i}^{-1} w+(2 N+2-J) x_{i}^{-1} w\right)$

$=-y\left(-2 \sum_{t=0}^{N}(t-1) x_{i}^{t-1} g_{t}-2 \sum_{t=0}^{N}(N+2-t) x_{i}^{t-1} g_{t}+J x_{i}^{-1} w+(2 N+2-J) x_{i}^{-1} w\right)$

$=-y\left(\sum_{t=0}^{N}(-2 N-2) x_{i}^{t-1} g_{t}+J x_{i}^{-1} w+(2 N+2-J) x_{i}^{-1} w\right)=0$.

Concluímos então que $f_{i}^{y} e_{-i} f_{i}^{-y}(w)=0$, de onde $e_{-i}\left(\sum_{t=0}^{N} x_{i}^{t-y} g_{t}\right)=0$. Logo, $v=\sum_{t=0}^{N} x_{i}^{t-y} g_{t}$ é um vetor $e_{-i^{-}}$primitivo tanto em $M_{i, \emptyset}$ como em $M_{i}$.

Como $N>J-i K-3$, então $2 N>J-i K-3+N$, o que implica $y>N$. Consequentemente, para $1 \leq t \leq N, y>t$, e temos um número inteiro positivo suficientemente grande, como desejado. 
Precisamos agora encontrar um vetor $e_{-i}$-primitivo em $M_{i, S}$, em que $S=\left\{j_{1}, \ldots, j_{l}\right\} \subset \mathbb{N}$ é um conjunto não vazio arbitrário. Notemos que, quando $S=\emptyset$, não nos preocupamos com a parcela $2 K \sum_{m>0} m \partial_{y_{m}} \partial_{x_{m+i}}$ em $e_{-i}$. Para o caso atual, devemos fazer escolhas convenientes para os índices dos monômios em $g_{0}$, de modo que essa parcela continue não interferindo, e possamos repetir as mesmas ideias utilizadas no caso anterior. Procedendo de modo análogo ao que fizemos no Lema 5.4.2, escolhemos $g_{0} \in \mathbb{C}[\mathbf{x}], g_{0}=x_{i_{1}} \ldots x_{i_{N+2}}-x_{i_{1}^{\prime}} \ldots x_{i_{N+2}^{\prime}}$, em que $i_{1}, \ldots, i_{N+2}, i_{1}^{\prime}, \ldots, i_{N+2}^{\prime}$ são todos maiores do que $i+\max \left\{j_{1}, \ldots, j_{l}\right\}$, tal que $\sum_{s=1}^{N+2} i_{s}=\sum_{s=1}^{N+2} i_{s}^{\prime}$ e $\varepsilon_{i}^{N+1}\left(g_{0}\right)=0$. Assim, substituindo $\sum_{t=0}^{N} x_{i}^{t} g_{t}$ por $y_{j_{1}}^{-1} \ldots y_{j_{l}}^{-1} \sum_{t=0}^{N} x_{i}^{t} g_{t}$ e repetindo os passos que foram feitos no caso anterior, teremos que $v=y_{j_{1}}^{-1} \ldots y_{j_{l}}^{-1} \sum_{t=0}^{N} x_{i}^{t-y} g_{t}$ é um vetor $e_{-i^{-}}$primitivo em $M_{i, S}$.

Corolário 5.4.5. Seja $J-i K$ um número inteiro. Então, ambos os módulos, $M_{i, S}(K \neq 0) e$ $M_{i}(K=0)$ são redutiveis.

Demonstração. Seja $K \neq 0$. Suponha, por absurdo, que $M_{i, S}$ seja irredutível. Consideremos então o espaço vetorial $T_{i, S}$ de todos os elementos de $M_{i, S}$ nos quais $e_{-i}$ age de forma localmente nilpotente.

Por um lado, de acordo com [14], $e_{-i}$ age de forma ad-localmente nilpotente em $\mathfrak{g}$, ou seja, para cada $x \in \mathfrak{g}$, existe $m \in \mathbb{N}$ tal que $\operatorname{ad}\left(e_{-i}\right)^{m}(x)=0$; por outro, para cada $v \in T_{i, S}$, existe $k \in \mathbb{N}$ tal que $e_{-i}^{k}(v)=0$.

Assim, como $\mathfrak{U}$ é uma álgebra associativa, utilizando a fórmula

$$
\left(e_{-i}^{n} x\right)(v)=\sum_{p=0}^{n}\left(\begin{array}{l}
n \\
p
\end{array}\right)\left(a d\left(e_{-i}\right)^{n-p}(x)\right) e_{-i}^{p}(v),
$$

com $n=m+k+1$, teremos que $T_{i, S}$ é um submódulo de $M_{i, S}$.

Pela Proposição 5.4.3 (a), $T_{i, S}$ é não trivial. Consequentemente, a ação de $e_{-i}$ em todos os vetores de $M_{i, S}=T_{i, S}$ é localmente nilpotente.

Porém, para $t \in \mathbb{N}$ suficientemente grande, $e_{-i}^{n}\left(x_{i}^{-t} y_{j_{1}}^{-1} \ldots y_{j_{l}}^{-1}\right) \neq 0$, para cada $n \geq 1$.

De fato,

$$
\begin{aligned}
e_{-i}\left(x_{i}^{-t} y_{j_{1}}^{-1} \ldots y_{j_{l}}^{-1}\right) & =-t(t+1+J-i K) x_{i}^{-t-1} y_{j_{1}}^{-1} \ldots y_{j_{l}}^{-1} \\
e_{-i}^{2}\left(x_{i}^{-t} y_{j_{1}}^{-1} \ldots y_{j_{l}}^{-1}\right) & =-t(t+1+J-i K)(-t-1)(J-i K+t+2) x_{i}^{-t-2} y_{j_{1}}^{-1} \ldots y_{j_{l}}^{-1} ; \\
\vdots & \\
e_{-i}^{n}\left(x_{i}^{-t} y_{j_{1}}^{-1} \ldots y_{j_{l}}^{-1}\right) & =(-t)(J-i K+t+1) \ldots(-t-n)(J-i K+t+n) x_{i}^{-t-n} y_{j_{1}}^{-1} \ldots y_{j_{l}}^{-1},
\end{aligned}
$$

para qualquer $n \geq 1$. Assim, se escolhermos, por exemplo, $t>-(J-i K)$, teremos o resultado desejado. Portanto, temos uma contradição, e $M_{i, S}$ é redutível.

De modo análogo, aplicando a Proposição 5.4.3 (b), mostra-se que $M_{i}$ é redutível. 


\subsubsection{O Caso $K \neq 0$}

Nesta subseção, provaremos as condições necessárias e suficientes para que os módulos $M_{S}$ e $M_{i, S}$ sejam irredutíveis, que é o item $(a)$ do Teorema 5.3.3.

Em primeiro lugar, notemos que se $K=0$, então ambos os módulos são redutíveis. Isso segue do fato de que o submódulo gerado por $y_{k_{1}}^{-1} \ldots y_{k_{n}}^{-1} y_{j}$ (respectivamente $x_{i}^{-1} y_{k_{1}}^{-1} \ldots y_{k_{n}}^{-1} y_{j}$ ), para algum $j \notin S$, é um submódulo próprio não trivial de $M_{S}$ (respectivamente $M_{i, S}$ ). Perceber que é um submódulo não trivial, é imediato.

Para mostrar que é próprio, observamos que como $K=0$, não é possível gerar potências de $y_{k_{t}}$ com expoentes negativos diferentes de -1 , para nenhum $t=1, \ldots, n$, já que a única parcela que poderia contribuir para isso é $\delta_{n>0} 2 n K \partial_{y_{n}}$.

Em seguida, recordamos que, pelo Corolário 5.4.5, $M_{i, S}$ é redutível se $J-i K \in \mathbb{Z}$. Assim, para mostrar o item (a) do teorema, precisamos analisar o que ocorre com $M_{S}$ no caso em que $K \neq 0$, e o que ocorre com $M_{i, S}$ no caso em que $K \neq 0$ e $J-i K \notin \mathbb{Z}$. Consequentemente, o Teorema 5.3.3 (a) segue imediatamente da seguinte proposição:

Proposição 5.4.6. Seja $K \neq 0$. Então, temos o seguinte:

(a) (i) $\mathfrak{U}\left(y_{k_{1}}^{-1} \ldots y_{k_{n}}^{-1}\right)=M_{S}$;

(ii) Seja $v \in M_{S}$ não nulo. Então, existe $u \in \mathfrak{U}$ tal que $u(v)=y_{k_{1}}^{-1} \ldots y_{k_{n}}^{-1}$.

(b) Seja $K \neq 0$ e $J-i K \notin \mathbb{Z}$. Então,

(i) $\mathfrak{U}\left(x_{i}^{-1} y_{k_{1}}^{-1} \ldots y_{k_{n}}^{-1}\right)=M_{i, S}$;

(ii) Seja $v \in M_{i, S}$ não nulo. Então existe $u \in \mathfrak{U}$ tal que $u(v)=x_{i}^{-1} y_{k_{1}}^{-1} \ldots y_{k_{n}}^{-1}$.

\subsubsection{Demonstração da Proposição 5.4.6(a)(i)}

Lema 5.4.7. Seja $v=y_{k_{1}}^{-\gamma_{1}} \ldots y_{k_{n}}^{-\gamma_{n}} y_{j_{1}}^{\beta_{1}} \ldots y_{j_{l}}^{\beta_{l}} \in M_{S}$ e seja $s>0$. Então, existe $u_{1} \in \mathfrak{U}$ tal que $u_{1}(v)=y_{s} v$. Além disso, se $s \in\left\{k_{1}, \ldots, k_{n}, j_{1}, \ldots, j_{l}\right\}$, então existe $u_{2} \in \mathfrak{U}$ tal que $u_{2}(v)=y_{s}^{-1} v$.

Demonstração. Como $-s<0$, e $v$ é um monômio apenas nas indeterminadas $y_{n}, n \in \mathbb{N}$, então $h_{-s}(v)=y_{-(-s)} v=y_{s} v$. Por outro lado, usando ainda o fato de que $v$ é um monômio onde não aparece nenhuma das indeterminadas $x_{m}, m \in \mathbb{Z}$, se $s \in\left\{k_{1}, \ldots, k_{n}, j_{1}, \ldots, j_{l}\right\}$, então

$$
h_{s}(v)=2 s K \partial_{y_{s}}(v)=c_{s} y_{s}^{-1} v
$$

em que

$$
c_{s}:=\left\{\begin{array}{cc}
-2 k_{t} \gamma_{t} K, & \text { se } s=k_{t}, \\
2 j_{t} \beta_{t} K, & \text { se } s=j_{t}, \\
0, & \text { caso contrário. }
\end{array}\right.
$$


Definimos então $u_{1}=h_{-s}$; e se $s \in\left\{k_{1}, \ldots, k_{n}, j_{1}, \ldots, j_{l}\right\}, u_{2}=\frac{1}{c_{s}} h_{s}$.

Demonstraremos agora a Proposição 5.4.6(a)(i): para cumprir nosso objetivo, mostraremos que cada monômio em $M_{S}$ pode ser obtido de $y_{k_{1}}^{-1} \ldots y_{k_{n}}^{-1}$ através da ação de algum elemento $u \in \mathfrak{U}$. Consideremos então

$$
u^{\prime}=f_{i_{k}}^{\alpha_{k}} \ldots f_{i_{1}}^{\alpha_{1}} h_{k_{1}}^{\gamma_{1}-1} \ldots h_{k_{n}}^{\gamma_{n}-1} h_{-j_{1}}^{\beta_{1}} \ldots h_{-j_{l}}^{\beta_{l}} \in \mathfrak{U}
$$

em que $\gamma_{i} \geq 1$, para todo $i$. Vamos analisar $u^{\prime}\left(y_{k_{1}}^{-1} \ldots y_{k_{n}}^{-1}\right)$ :

$$
h_{-j_{1}}^{\beta_{1}} \ldots h_{-j_{l}}^{\beta_{l}}\left(y_{k_{1}}^{-1} \ldots y_{k_{n}}^{-1}\right)=y_{j_{1}}^{\beta_{1}} \ldots y_{j_{l}}^{\beta_{l}} y_{k_{1}}^{-1} \ldots y_{k_{n}}^{-1},
$$

pois a ação de $h_{-j_{t}}$ em $y_{k_{1}}^{-1} \ldots y_{k_{n}}^{-1}$ é a multiplicação por $y_{j_{t}}$, para $t=1, \ldots, l$. Em seguida, utilizando a demonstração do Lema 5.4.7, a ação de $h_{k_{t}}$, para $t=1, \ldots, n$ é a multiplicação por $c_{k_{t}} y_{k_{t}}^{-1}$, o que fornece

$$
h_{k_{1}}^{\gamma_{1}-1} \ldots h_{k_{n}}^{\gamma_{n}-1}\left(y_{j_{1}}^{\beta_{1}} \ldots y_{j_{l}}^{\beta_{l}} y_{k_{1}}^{-1} \ldots y_{k_{n}}^{-1}\right)=A y_{k_{1}}^{-\gamma_{1}} \ldots y_{k_{n}}^{-\gamma_{n}} y_{j_{1}}^{\beta_{1}} \ldots y_{j_{l}}^{\beta_{l}}
$$

em que $A \in \mathbb{C}$ e $A \neq 0$. Por fim, como $f_{i_{t}}$ fornece apenas a multiplicação por $x_{i_{t}}$, para $t=1, \ldots, k$,

$$
f_{i_{k}}^{\alpha_{k}} \ldots f_{i_{1}}^{\alpha_{1}}\left(A y_{k_{1}}^{-\gamma_{1}} \ldots y_{k_{n}}^{-\gamma_{n}} y_{j_{1}}^{\beta_{1}} \ldots y_{j_{l}}^{\beta_{l}}\right)=A y_{k_{1}}^{-\gamma_{1}} \ldots y_{k_{n}}^{-\gamma_{n}} y_{j_{1}}^{\beta_{1}} \ldots y_{j_{l}}^{\beta_{l}} x_{i_{1}}^{\alpha_{1}} \ldots x_{i_{k}}^{\alpha_{k}}
$$

e fazendo $u=\frac{1}{A} u^{\prime}$, temos a igualdade

$$
u\left(y_{k_{1}}^{-1} \ldots y_{k_{n}}^{-1}\right)=y_{k_{1}}^{-\gamma_{1}} \ldots y_{k_{n}}^{-\gamma_{n}} y_{j_{1}}^{\beta_{1}} \ldots y_{j_{l}}^{\beta_{l}} x_{i_{1}}^{\alpha_{1}} \ldots x_{i_{k}}^{\alpha_{k}}
$$

de onde segue que qualquer monômio pode ser obtido escolhendo expoentes convenientes no elemento $u^{\prime} \in \mathfrak{U}$. Portanto, como cada elemento em $M_{S}$ pode ser escrito como uma soma finita de monômios, $\mathfrak{U}\left(y_{k_{1}}^{-1} \ldots y_{k_{n}}^{-1}\right)=M_{S}$.

\subsubsection{Demonstração da Proposição 5.4.6(a)(ii)}

Lema 5.4.8. Seja $v$ um vetor não nulo em $M_{S}$ tal que $\operatorname{deg}_{x}(v)=0$. Então, existe $u \in \mathfrak{U}$ tal que $u(v)=y_{k_{1}}^{-1} \ldots y_{k_{n}}^{-1}$.

Demonstração. Seja $v \in M_{S}$ um vetor não nulo. Como, por hipótese, $\operatorname{deg}_{x}(v)=0$, então $v$ é um polinômio cujos monômios são da forma

$$
w=y_{k_{1}}^{-\gamma_{1}} \ldots y_{k_{n}}^{-\gamma_{n}} y_{j_{1}}^{\beta_{1}} \ldots y_{j_{l}}^{\beta_{l}}
$$


Como $v$ é uma soma finita de monômios da forma (5.6), digamos $v=w_{1}+\ldots+w_{m}$, temos condições de aplicar o Lema 5.4.7 a cada um deles.

Em primeiro lugar, para $y_{k_{1}}$, comparando os expoentes $\gamma_{1}$ dentre todos os monômios, consideremos o maior deles em módulo e denotemos por $\gamma_{1_{\max }}$. Aplicando o Lema 5.4.7 sucessivamente, temos

$$
h_{-k_{1}}^{\gamma_{1 \max }-1}(v)=h_{-k_{1}}^{\gamma_{1 \max }-1}\left(w_{1}+\ldots+w_{m}\right)=h_{-k_{1}}^{\gamma_{1 \max }-1}\left(w_{1}\right)+\ldots+h_{-k_{1}}^{\gamma_{1 \max }-1}\left(w_{m}\right),
$$

e obtemos um polinômio não nulo no qual cada monômio possui a potência $y_{k_{1}}^{-1}$.

Podemos repetir o processo sucessivamente, até fixarmos $y_{k_{n}}$ e obtermos um elemento não nulo $v_{1}=y_{k_{1}}^{-1} \ldots y_{k_{n}}^{-1} g(\mathbf{y}) \in M_{S}$, em que $g$ é um polinômio satisfazendo $\partial_{y_{k_{t}}}(g)=0$, para todo $t=1, \ldots, n$.

Se $\operatorname{deg}_{y}(g)=0$, então $v_{1}$ não possui nenhuma das indeterminadas $y_{j}, j \notin S$, e temos o elemento $u \in \mathfrak{U}$ que satisfaz o desejado,

$$
u=h_{-k_{1}}^{\gamma_{1} \max ^{-1}} \ldots h_{-k_{n}}^{\gamma_{n_{\max }}-1}
$$

Se $\operatorname{deg}_{y}(g)>0$, existe $s \in \mathbb{N}$ tal que $\partial_{y_{s}}(g) \neq 0$, e notemos que $s \neq k_{t}$, para todo $t=1, \ldots, n$. Com $s$ fixado, vamos avaliar $h_{s}\left(v_{1}\right)$ :

$$
h_{s}\left(v_{1}\right)=2 s K \partial_{y_{s}}\left(v_{1}\right)=2 s K y_{k_{1}}^{-1} \ldots y_{k_{n}}^{-1} \partial_{y_{s}}(g)=A y_{k_{1}}^{-1} \ldots y_{k_{n}}^{-1} y_{s}^{-1} g
$$

em que $A \in \mathbb{C}, A \neq 0$.

Com essas informações, e tomando $\operatorname{deg}_{y}(g)=0$ como base, a demonstração é feita por indução em $\operatorname{deg}_{y}(g)$.

Assim, se $\operatorname{deg}_{y}(g)=k>0$, vamos assumir como hipótese de indução que sempre que existir $u^{\prime} \in \mathfrak{U} \operatorname{com} u^{\prime}(v)=y_{k_{1}}^{-1} \ldots y_{k_{n}}^{-1} g^{\prime}(\mathbf{y})$, para algum polinômio $g^{\prime}$ tal que $\partial_{y_{k_{t}}}\left(g^{\prime}\right)=0$, para cada $t=1, \ldots, n$, e $\operatorname{deg}_{y}\left(g^{\prime}\right)<k$, podemos encontrar $u \in \mathfrak{U}$ tal que $u u^{\prime}(v)=y_{k_{1}}^{-1} \ldots y_{k_{n}}^{-1}$.

Portanto, utilizando (5.7), e tomando $u^{\prime}=\frac{1}{A} h_{s} h_{-k_{1}}^{\gamma_{1 \text { max }}-1} \ldots h_{-k_{n}}^{\gamma_{n_{\max }}-1}$, temos o resultado desejado.

Lema 5.4.9. Seja $v \in N_{S}$. Então, para cada $j \in \mathbb{Z}$, existe $n>0$ suficientemente grande tal que

$$
e_{-n}(v)=y_{n-j} \partial_{x_{j}}(v)+v^{\prime},
$$

para algum $v^{\prime} \in N_{S}$ tal que $\partial_{y_{n-j}}\left(v^{\prime}\right)=0$.

Demonstração. Em primeiro lugar, notemos que $v$ é um polinômio formado por monômios da forma

$$
w=x_{i_{1}}^{\alpha_{1}} \ldots x_{i_{k}}^{\alpha_{k}} y_{k_{1}}^{\gamma_{1}} \ldots y_{k_{n}}^{\gamma_{n}} y_{j_{1}}^{\beta_{1}} \ldots y_{j_{l}}^{\beta_{l}} .
$$

Fixado $j \in \mathbb{Z}$, consideremos $n>0$, com $n>j$, e teremos: 


$$
\begin{aligned}
e_{-n}(v) & =-\sum_{m, k \in \mathbb{Z}} x_{k+m-n} \partial_{x_{k}} \partial_{x_{m}}(v)+\sum_{k>0} y_{k} \partial_{x_{-k+n}}(v)+2 K \sum_{m>0} m \partial_{y_{m}} \partial_{x_{m+n}}(v) \\
& +(J-n K) \partial_{x_{n}}(v) \\
& =-\sum_{m, k \in \mathbb{Z}} x_{k+m-n} \partial_{x_{k}} \partial_{x_{m}}(v)+y_{n-j} \partial_{x_{j}}(v)+\sum_{\substack{k>0 \\
k \neq n-j}} y_{k} \partial_{x_{-k+n}}(v) \\
& +2 K \sum_{m>0} m \partial_{y_{m}} \partial_{x_{m+n}}(v)+(J-n K) \partial_{x_{n}}(v) .
\end{aligned}
$$

Tomando

$$
v^{\prime \prime}=-\sum_{m, k \in \mathbb{Z}} x_{k+m-n} \partial_{x_{k}} \partial_{x_{m}}(v)+\sum_{\substack{k>0 \\ k \neq n-j}} y_{k} \partial_{x_{-k+n}}(v)+2 K \sum_{m>0} m \partial_{y_{m}} \partial_{x_{m+n}}(v)+(J-n K) \partial_{x_{n}}(v),
$$

temos $e_{-n}(v)=y_{n-j} \partial_{x_{j}}(v)+v^{\prime \prime}, \operatorname{com} v^{\prime \prime} \in N_{S}$.

Se $\partial_{y_{n-j}}\left(v^{\prime \prime}\right)=0$, já temos $v^{\prime}=v^{\prime \prime} \in N_{S}$ com as propriedades desejadas.

Veremos agora o que ocorre quando $\partial_{y_{n-j}}\left(v^{\prime \prime}\right) \neq 0$. Nesse caso, notemos que $\partial_{y_{n-j}}(v) \neq 0$, pois a única parcela de $e_{-n}(v)$ que poderia produzir $y_{n-j}$ não aparece em $v^{\prime \prime}$. Assim, a única ferramenta que temos é o controle da escolha de $n>0$.

Como $v$ é uma soma finita de monômios da forma (5.8), digamos $v=w_{1}+\ldots+w_{q}$, podemos escolher $n>0$ da seguinte forma: em primeiro lugar, como $k_{1}, \ldots, k_{n}$ são números naturais fixos, tomamos $m=\max \left\{j+k_{t}, t=1, \ldots, n\right\}$; em seguida, para cada monômio $w_{r}$, toma$\operatorname{mos} m_{r}=\max \left\{j+j_{p}, p=1, \ldots, l\right\}$; por fim, tomando $m^{\prime}=\max \left\{m_{r}, r=1, \ldots, q\right\}$, escolhemos $n>\max \left\{m, m^{\prime}\right\}$, e teremos o resultado desejado.

Demonstraremos agora a Proposição 5.4.6(a)(ii) por indução em $\operatorname{deg}_{x}(v)$, em que a base será $\operatorname{deg}_{x}(v)=0$. A validade deste caso é uma consequência imediata do Lema 5.4.8.

Suponhamos agora que $\operatorname{deg}_{x}(v)=k \geq 1$, e fixemos $j \in \mathbb{Z}$ tal que $\partial_{x_{j}}(v) \neq 0$.

Pelo Lema 5.4.9, existe $n>0$ suficientemente grande tal que $e_{-n}(v) \neq 0$ em $M_{S}$. Como hipótese de indução, vamos supor que para todo $v \in M_{S}$, não nulo, $\operatorname{com} \operatorname{deg}_{x}(v)<k$, exista $u \in \mathfrak{U}$ tal que $u(v)=y_{k_{1}}^{-1} \ldots y_{k_{n}}^{-1}$. Queremos mostrar que o mesmo é válido para $\operatorname{deg}_{x}(v)=k$. Notemos que, em particular, $\operatorname{deg}_{x}\left(e_{-n}(v)\right)<\operatorname{deg}_{x}(v)=k$. Assim, pela hipótese de indução, existe $u \in \mathfrak{U}$ tal que $u\left(e_{-n}(v)\right)=y_{k_{1}}^{-1} \ldots y_{k_{n}}^{-1}$, e $u e_{-n} \in \mathfrak{U}$ é o elemento desejado.

\subsubsection{Demonstração da Proposição 5.4.6(b) $(i)$}

Lema 5.4.10. Seja $J-i K \notin \mathbb{Z}$ e $v=x_{i}^{-p} y_{k_{1}}^{-\gamma_{1}} \ldots y_{k_{n}}^{-\gamma_{n}} y_{j_{1}}^{\beta_{1}} \ldots y_{j_{l}}^{\beta_{l}} \in M_{i, S}$. Seja $s>0$. Então, existem $u_{1}, u_{2} \in \mathfrak{U}$ tais que $u_{1}(v)=x_{i}^{-1} v$ e $u_{2}(v)=y_{s} v$. Além disso, se $s \in\left\{k_{1}, \ldots, k_{n}, j_{1}, \ldots, j_{l}\right\}$, então existe $u_{3} \in \mathfrak{U}$ tal que $u_{3}(v)=y_{s}^{-1} v$.

Demonstração. Sejam 


$$
u_{1}=\frac{-1}{p(p+1+J-i K)} e_{-i}, u_{2}=h_{-s}+\frac{2}{p+1+J-i K} f_{i-s} e_{-i},
$$

e para $s \in\left\{k_{1}, \ldots, k_{n}, j_{1}, \ldots, j_{l}\right\}$, consideremos

$$
u_{3}=\frac{1}{c_{s}}\left(h_{s}+\frac{2}{p+1+J-i K} f_{i+s} e_{-i}\right)
$$

em que $c_{s} \neq 0$ foi definido em (5.5).

Notemos que $p \neq 0$, pois $v \in M_{i, S}$; e também $p+1+J-i K \neq 0$, pois $j-i K \notin \mathbb{Z} \mathrm{e}-(p+1) \in \mathbb{Z}$. Mostraremos que $u_{1}, u_{2}, u_{3}$ satisfazem as propriedades desejadas.

Como

$$
e_{-i}(v)=-x_{2 i-i} \partial_{x_{i}}^{2}(v)+(J-i K) \partial_{x_{i}}(v)=-x_{i}(-p)(-p-1) x_{i}^{-2} v+(J-i K)(-p) x_{i}^{-1} v,
$$

então

$$
\begin{aligned}
u_{1}(v) & =\frac{-1}{p(p+1+J-i K)}\left(p(-p-1) x_{i}^{-1} v-p(J-i K) x_{i}^{-1} v\right) \\
& =\frac{1}{p(p+1+J-i K)}(p(p+1+J-i K)) x_{i}^{-1} v=x_{i}^{-1} v
\end{aligned}
$$

Agora, para $u_{2}(v)$, vamos utilizar $e_{-i}(v)=-p(p+1+J-i K) x_{i}^{-1} v$. Assim,

$$
f_{i-s} e_{-i}(v)=-p(p+1+J-i K) x_{i-s} x_{i}^{-1} v \text { e } \frac{2}{p+1+J-i K} f_{i-s} e_{-i}(v)=-2 p x_{i-s} x_{i}^{-1} v .
$$

Como $h_{-s}(v)=-2 x_{i-s} \partial_{x_{i}}(v)+y_{s} v$, então

$$
u_{2}(v)=-2 x_{i-s}(-p) x_{i}^{-1} v+y_{s} v+2(-p) x_{i-s} x_{i}^{-1} v=y_{s} v
$$

Finalmente, avaliamos $u_{3}(v)$ : novamente, já temos $e_{-i}(v)=-p(p+1+J-i K) x_{i}^{-1} v$. Assim, $f_{i+s} e_{-i}(v)=-p(p+1+J-i K) x_{i+s} x_{i}^{-1} v$. Consequentemente,

$$
\frac{2}{p+1+J-i K} f_{i+s} e_{-i}(v)=-2 p x_{i+s} x_{i}^{-1} v
$$

Por outro lado, se $s \in\left\{k_{1}, \ldots, k_{n}, j_{1}, \ldots, j_{l}\right\}$,

$$
h_{s}(v)=-2 x_{i+s} \partial_{x_{i}}(v)+2 s K \partial_{y_{s}}(v)=-2 x_{i+s}(-p) x_{i}^{-1} v+c_{s} y_{s}^{-1} v
$$

Portanto, 


$$
\begin{aligned}
u_{3}(v) & =\frac{1}{c_{s}}\left(h_{s}+\frac{2}{p+1+J-i K} f_{i+s} e_{-i}\right)(v) \\
& =\frac{1}{c_{s}}\left(-2 x_{i+s}(-p) x_{i}^{-1} v+c_{s} y_{s}^{-1} v-2 p x_{i+s} x_{i}^{-1} v\right)=\frac{1}{c_{s}} c_{s} y_{s}^{-1} v=y_{s}^{-1} v
\end{aligned}
$$

Demonstraremos agora a Proposição 5.4.6(b)(i): De modo análogo ao que fizemos na demonstração do item $(a)(i)$, mostraremos que cada monômio não nulo em $M_{i, S}$ pode ser obtido através da ação de algum elemento $u \in \mathfrak{U}$ em $x_{i}^{-1} y_{k_{1}}^{-1} \ldots y_{k_{n}}^{-1}$, e usaremos o fato de que cada polinômio em $M_{i, S}$ é uma soma finita de monômios. Consideremos o monômio

$$
w=x_{i}^{-p} y_{k_{1}}^{-\gamma_{1}} \ldots y_{k_{n}}^{-\gamma_{n}} y_{j_{1}}^{\beta_{1}} \ldots y_{j_{l}}^{\beta_{l}} x_{i_{1}}^{\alpha_{1}} \ldots x_{i_{k}}^{\alpha_{k}} \in M_{i, S}
$$

Encontraremos $u \in \mathfrak{U}$ tal que $u\left(x_{i}^{-1} y_{k_{1}}^{-1} \ldots y_{k_{n}}^{-1}\right)=w$.

Claramente, podemos aplicar o Lema 5.4 .10 para $v=x_{i}^{-1} y_{k_{1}}^{-1} \ldots y_{k_{n}}^{-1}$. Assim, fixando $t \in\{1, \ldots, l\}$, e fazendo $s=j_{t}$,

$$
u_{2}\left(x_{i}^{-1} y_{k_{1}}^{-1} \ldots y_{k_{n}}^{-1}\right)=y_{j_{t}} x_{i}^{-1} y_{k_{1}}^{-1} \ldots y_{k_{n}}^{-1} \in M_{i, S}
$$

O monômio obtido ainda satisfaz as condições para a utilização do Lema 5.4.10, e assim sucessivamente, e teremos

$$
u_{2}^{\beta_{t}}\left(x_{i}^{-1} y_{k_{1}}^{-1} \ldots y_{k_{n}}^{-1}\right)=x_{i}^{-1} y_{k_{1}}^{-1} \ldots y_{k_{n}}^{-1} y_{j_{t}}^{\beta_{t}} \in M_{i, S}
$$

Para cada $t=1, \ldots, l$ fixado, denotando $u_{2}$ por $u_{t_{2}}$, teremos

$$
u_{1_{2}}^{\beta_{1}} \ldots u_{l_{2}}^{\beta_{l}}\left(x_{i}^{-1} y_{k_{1}}^{-1} \ldots y_{k_{n}}^{-1}\right)=x_{i}^{-1} y_{k_{1}}^{-1} \ldots y_{k_{n}}^{-1} y_{j_{1}}^{\beta_{1}} \ldots y_{j_{l}}^{\beta_{l}} \in M_{i, S}
$$

Aplicaremos agora o Lema 5.4 .10 para $x_{i}^{-1} y_{k_{1}}^{-1} \ldots y_{k_{n}}^{-1} y_{j_{1}}^{\beta_{1}} \ldots y_{j_{l}}^{\beta_{l}} \in M_{i, S}$. De modo análogo ao que acabamos de fazer, fixando $t \in\{1, \ldots, k\}$ e fazendo $s=k_{t}$,

$$
u_{3}\left(x_{i}^{-1} y_{k_{1}}^{-1} \ldots y_{k_{n}}^{-1} y_{j_{1}}^{\beta_{1}} \ldots y_{j_{l}}^{\beta_{l}}\right)=y_{k_{t}}^{-1} x_{i}^{-1} y_{k_{1}}^{-1} \ldots y_{k_{n}}^{-1} y_{j_{1}}^{\beta_{1}} \ldots y_{j_{l}}^{\beta_{l}} \in M_{i, S}
$$

O monômio obtido ainda satisfaz as condições para a utilização do Lema 5.4.10, e assim sucessivamente, e teremos

$$
u_{3}^{\gamma_{t}-1}\left(x_{i}^{-1} y_{k_{1}}^{-1} \ldots y_{k_{n}}^{-1} y_{j_{1}}^{\beta_{1}} \ldots y_{j_{l}}^{\beta_{l}}\right)=y_{k_{t}}^{-\gamma_{t}} x_{i}^{-1} y_{k_{1}}^{-1} \ldots y_{k_{n}}^{-1} y_{j_{1}}^{\beta_{1}} \ldots y_{j_{l}}^{\beta_{l}} \in M_{i, S},
$$

com $\gamma_{t} \geq 1$. Para cada $t=1, \ldots, n$ fixado, denotando $u_{3}$ por $u_{t_{3}}$, teremos 


$$
u_{1_{3}}^{\gamma_{1}-1} \ldots u_{n_{3}}^{\gamma_{n}-1}\left(x_{i}^{-1} y_{k_{1}}^{-1} \ldots y_{k_{n}}^{-1} y_{j_{1}}^{\beta_{1}} \ldots y_{j_{l}}^{\beta_{l}}\right)=x_{i}^{-1} y_{k_{1}}^{-\gamma_{1}} \ldots y_{k_{n}}^{-\gamma_{n}} y_{j_{1}}^{\beta_{1}} \ldots y_{j_{l}}^{\beta_{l}} \in M_{i, S}
$$

Aplicando agora o Lema ao elemento obtido acima,

$$
u_{1}\left(x_{i}^{-1} y_{k_{1}}^{-\gamma_{1}} \ldots y_{k_{n}}^{-\gamma_{n}} y_{j_{1}}^{\beta_{1}} \ldots y_{j_{l}}^{\beta_{l}}\right)=x_{i}^{-1} x_{i}^{-1} y_{k_{1}}^{-\gamma_{1}} \ldots y_{k_{n}}^{-\gamma_{n}} y_{j_{1}}^{\beta_{1}} \ldots y_{j_{l}}^{\beta_{l}} \in M_{i, S}
$$

Portanto, como as condições para aplicações sucessivas são satisfeitas,

$$
u_{1}^{p-1}\left(x_{i}^{-1} y_{k_{1}}^{-\gamma_{1}} \ldots y_{k_{n}}^{-\gamma_{n}} y_{j_{1}}^{\beta_{1}} \ldots y_{j_{l}}^{\beta_{l}}\right)=x_{i}^{-p} y_{k_{1}}^{-\gamma_{1}} \ldots y_{k_{n}}^{-\gamma_{n}} y_{j_{1}}^{\beta_{1}} \ldots y_{j_{l}}^{\beta_{l}} \in M_{i, S}
$$

$p \geq 1$.

Observando que para cada $t=1, \ldots, k$, a ação de $f_{i_{t}}$ em qualquer elemento de $M_{i, S}$ é a multiplicação por $x_{i_{t}}$, aplicações sucessivas fornecem

$$
f_{i_{t}}^{\alpha_{t}}\left(x_{i}^{-p} y_{k_{1}}^{-\gamma_{1}} \ldots y_{k_{n}}^{-\gamma_{n}} y_{j_{1}}^{\beta_{1}} \ldots y_{j_{l}}^{\beta_{l}}\right)=x_{i}^{-p} y_{k_{1}}^{-\gamma_{1}} \ldots y_{k_{n}}^{-\gamma_{n}} y_{j_{1}}^{\beta_{1}} \ldots y_{j_{l}}^{\beta_{l}} x_{i_{t}}^{\alpha_{t}} \in M_{i, S}
$$

Consequentemente,

$$
f_{i_{1}}^{\alpha_{1}} \ldots f_{i_{k}}^{\alpha_{k}}\left(x_{i}^{-p} y_{k_{1}}^{-\gamma_{1}} \ldots y_{k_{n}}^{-\gamma_{n}} y_{j_{1}}^{\beta_{1}} \ldots y_{j_{l}}^{\beta_{l}}\right)=x_{i}^{-p} y_{k_{1}}^{-\gamma_{1}} \ldots y_{k_{n}}^{-\gamma_{n}} y_{j_{1}}^{\beta_{1}} \ldots y_{j_{l}}^{\beta_{l}} x_{i_{1}}^{\alpha_{1}} \ldots x_{i_{k}}^{\alpha_{k}} \in M_{i, S}
$$

Concluímos então que $u=f_{i_{1}}^{\alpha_{1}} \ldots f_{i_{k}}^{\alpha_{k}} u_{1}^{p-1} u_{1_{3}}^{\gamma_{1}-1} \ldots u_{n_{3}}^{\gamma_{n}-1} u_{1_{2}}^{\beta_{1}} \ldots u_{l_{2}}^{\beta_{l}} \in \mathfrak{U}$ é o elemento desejado e $\mathfrak{U}\left(x_{i}^{-1} y_{k_{1}}^{-1} \ldots y_{k_{n}}^{-1}\right)=M_{i, S}$.

\subsubsection{Demonstração da Proposição 5.4.6(b) $(i i)$}

Lema 5.4.11. Seja $J-i K \notin \mathbb{Z}$ e v um vetor não nulo em $M_{i, S}$ tal que $\operatorname{deg}_{x^{+}}(v)=0$. Então, existe $u \in \mathfrak{U}$ tal que $u(v)=x_{i}^{-1} y_{k_{1}}^{-1} \ldots y_{k_{n}}^{-1}$.

Demonstração. Seja $v \in M_{i, S}$ um vetor não nulo tal que $\operatorname{deg}_{x^{+}}(v)=0$. Então, $v$ pode ser escrito como uma soma finita de monômios $v=w_{1}+\ldots+w_{r}$, em que cada monômio é da forma

$$
w_{t}=x_{i}^{-p_{t}} y_{k_{1}}^{-\gamma_{1 t}} \ldots y_{k_{n}}^{-\gamma_{n t}} y_{j_{1 t}}^{\beta_{1 t}} \ldots y_{j_{l t}}^{\beta_{l t}} \in M_{i, S},
$$

com $t=1, \ldots, r$.

Seja agora $\gamma_{1_{\max }}=\max \left\{\left|\gamma_{1 t}\right|, t=1, \ldots, r\right\}$. De acordo com o Lema 5.4.10, existe $u_{1_{2}} \in \mathfrak{U}$ tal que $u_{1_{2}}\left(w_{t}\right)=y_{k_{1}} w_{t}$, para todo $t=1, \ldots, r$. Assim, podemos considerar $u_{1_{2}}(v)$, de onde obtemos um novo polinômio em $M_{i, S}$, cujos monômios ainda satisfazem as condições para a aplicação do Lema 5.4.10. Com aplicações sucessivas, obtemos 


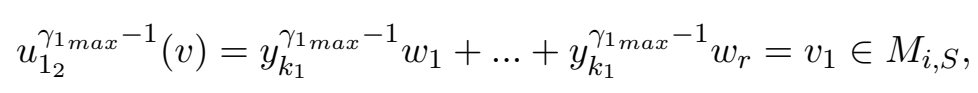

que pode ser reescrito como uma nova soma finita de monômios $v_{1}=w_{1}^{\prime}+\ldots+w_{r^{\prime}}^{\prime}$, em que $r^{\prime} \leq r$, e cada monômio é da forma

$$
w_{t^{\prime}}^{\prime}=x_{i}^{-p_{t^{\prime}}} y_{k_{1}}^{-1} \ldots y_{k_{n}}^{-\gamma_{n}} y_{j_{1^{\prime}}}^{\beta_{1^{\prime}}} \ldots y_{j_{t^{\prime}}}^{\beta_{t^{\prime}}} \in M_{i, S}
$$

com $t^{\prime}=1, \ldots, r^{\prime}$. Cada um desses novos monômios ainda satisfaz as condições para a aplicação do Lema 5.4.10, e podemos repetir o processo sucessivamente, até fixarmos $n$ e encontrar $u_{n_{2}} \in \mathfrak{U}$ tal que $u_{n_{2}}^{\gamma_{\text {max }}-1}\left(v_{n-1}\right)=v_{n}=q_{1}+\ldots+q_{s}$, em que cada monômio é da forma

$$
q_{t}=x_{i}^{-p_{t}} y_{k_{1}}^{-1} \ldots y_{k_{n}}^{-1} y_{j_{1 t}}^{\beta_{1 t}} \ldots y_{j_{l t}}^{\beta_{l t}} \in M_{i, S},
$$

$\operatorname{com} t=1, \ldots, s$.

Por fim, seja $p_{\max }=\max \left\{\left|p_{t}\right|, t=1, \ldots, s\right\}$, e teremos

$$
f_{i}^{p_{\max }-1}\left(v_{n}\right)=x_{i}^{p-1} v_{n}=x_{i}^{-1} y_{k_{1}}^{-1} \ldots y_{k_{n}}^{-1} g(\mathbf{y})
$$

em que $g(\mathbf{y})$ é um polinômio não nulo em $M_{i, S}$ satisfazendo $\partial_{y_{k_{t}}}(g)=0$, para todo $t=1, \ldots, n$, isto é, existe $u_{1} \in \mathfrak{U}$ tal que $u_{1}(v)=x_{i}^{-1} y_{k_{1}}^{-1} \ldots y_{k_{n}}^{-1} g(\mathbf{y})$.

Para concluir a demonstração, utilizaremos indução em $\operatorname{deg}_{y} g$.

Se $\operatorname{deg}_{y} g=0$, então $u_{1} \in \mathfrak{U}$ é o elemento desejado, e esta é a nossa base de indução. Seja agora $\operatorname{deg}_{y} g \geq 1$. Então, existe $j \in \mathbb{N}$ tal que $\partial_{y_{j}}(g) \neq 0$. Assim, considerando

$$
u_{j_{3}}=h_{j}+\frac{2}{2+J-i K} f_{i+j} e_{-i} \in \mathfrak{U}
$$

temos

$$
u_{j_{3}}\left(x_{i}^{-1} y_{k 1}^{-1} \ldots y_{k_{n}}^{-1} g\right)=2 j K \partial_{y_{j}}\left(x_{i}^{-1} y_{k 1}^{-1} \ldots y_{k_{n}}^{-1} g\right)=2 j K x_{i}^{-1} y_{k 1}^{-1} \ldots y_{k_{n}}^{-1} \partial_{y_{j}}(g)
$$

Por hipótese de indução, assumiremos que se existe $u_{4} \in \mathfrak{U}$, com $u_{4}(v)=x_{i}^{-1} y_{k_{1}}^{-1} \ldots y_{k_{n}}^{-1} g(\mathbf{y})$, em que $g(\mathbf{y})$ é um polinômio em $M_{i, S}$ satisfazendo $\partial_{y_{k_{t}}}(g)=0$, para todo $t=1, \ldots, n$ e $\operatorname{deg}_{y} g=k$, existe $u^{\prime} \in \mathfrak{U}$ tal que

$$
u^{\prime}\left(x_{i}^{-1} y_{k_{1}}^{-1} \ldots y_{k_{n}}^{-1} g\right)=x_{i}^{-1} y_{k_{1}}^{-1} \ldots y_{k_{n}}^{-1}
$$

Portanto, se $d e g_{y} g=k+1$, então 


$$
u_{j_{3}}\left(x_{i}^{-1} y_{k_{1}}^{-1} \ldots y_{k_{n}}^{-1} g\right)
$$

é um polinômio com $d e g_{y} g=k$, que satisfaz a hipótese de indução (basta fazer $u_{4}=u_{j_{3}} u_{1}$ ). Assim, podemos encontrar $u^{\prime} \in \mathfrak{U}$ que nos forneça o resultado desejado.

Definição 5.4.12. Para $p \in \mathbb{C}$ e $\alpha \in \mathbb{N}$, definimos:

$$
A_{\alpha, p}=-p(p+1-2 \alpha+J-i K)
$$

A definição acima é motivada pelo seguinte:

Lema 5.4.13. Seja $Z=y_{k_{1}}^{-\gamma_{1}} \ldots y_{k_{n}}^{-\gamma_{n}} y_{j_{1}}^{\beta_{1}} \ldots y_{j_{l}}^{\beta_{l}} x_{i_{1}}^{\alpha_{1}} \ldots x_{i_{k}}^{\alpha_{k}}$, com $p, \gamma_{1}, \ldots, \gamma_{n}, \beta_{1}, \ldots, \beta_{l}, \alpha_{1}, \ldots, \alpha_{k} \in \mathbb{N}$. Então,

$$
e_{-i}\left(x_{i}^{-p} Z\right)=A_{\alpha, p} x_{i}^{-p-1} Z+x_{i}^{-p} Z^{\prime}+x_{i}^{-p+1} Z^{\prime \prime}
$$

em $M_{i, S}$, em que $\alpha=\sum_{s=1}^{k} \alpha_{s}=\operatorname{deg}_{x^{+}}\left(x_{i}^{-p} Z\right)$, e $Z^{\prime}$ e $Z^{\prime \prime}$ são polinômios deg $x_{x^{+}}$-homogêneos, que são ou nulos em $M_{i, S}$ ou $\operatorname{deg}_{x^{+}}\left(x_{i}^{-p+1} Z^{\prime \prime}\right)=\operatorname{deg}_{x^{+}}\left(x_{i}^{-p} Z^{\prime}\right)=\alpha-1$. Em particular,

$$
f_{i} e_{-i}\left(x_{i}^{-1} Z\right)=A_{\alpha, 1} x_{i}^{-1} Z
$$

Demonstração. Inicialmente, recordemos $e_{-i}$ :

$$
e_{-i}=-\sum_{m, k \in \mathbb{Z}} x_{m+k-i} \partial_{x_{m}} \partial_{x_{k}}+\sum_{k>0} y_{k} \partial_{x_{-k+i}}+2 K \sum_{m>0} m \partial_{y_{m}} \partial_{x_{m+i}}+(J-i K) \partial_{x_{i}}
$$

e notemos que é possível reescrevê-lo como uma nova soma, em que possamos visualizar separadamente os operadores que dependem de $x_{i}$ e os que não dependem:

$$
\begin{aligned}
e_{-i} & =-x_{i} \partial_{x_{i}} \partial_{x_{i}}-\sum_{\substack{s_{1}, s_{2} \in \mathbb{Z} \backslash\{i\} \\
s_{1}+s_{2} \neq 2 i}} x_{s_{1}+s_{2}-i} \partial_{x_{s_{1}}} \partial_{x_{s_{2}}}-\sum_{\substack{s_{1}, s_{2} \in \mathbb{Z} \backslash\{i\} \\
s_{1}+s_{2}=2 i}} x_{s_{1}+s_{2}-i} \partial_{x_{s_{1}}} \partial_{x_{s_{2}}} \\
& -2\left(\sum_{s \in \mathbb{Z} \backslash\{i\}} x_{s+i-i} \partial_{x_{s}}\right) \partial_{x_{i}}+\sum_{s>0} y_{s} \partial_{x_{i-s}}+2 K \sum_{t>0} t \partial_{y_{t}} \partial_{x_{t+i}}+(J-i K) \partial_{x_{i}} .
\end{aligned}
$$

Assim, podemos apresentar $e_{-i}$ como uma soma de três operadores $d e g_{x^{+}}$-homogêneos

$$
e_{-i}=e_{-i, 0}+e_{-i, 1}+x_{i} e_{-i, 2}
$$


em que

$$
\begin{aligned}
& e_{-i, 0}=-x_{i} \partial_{x_{i}} \partial_{x_{i}}-2\left(\sum_{s \in \mathbb{Z} \backslash\{i\}} x_{s} \partial_{x_{s}}\right) \partial_{x_{i}}+(J-i K) \partial_{x_{i}}, \\
& e_{-i, 1}=\sum_{\substack{s_{1}, s_{2} \in \mathbb{Z} \backslash\{i\} \\
s_{1}+s_{2} \neq 2 i}} x_{s_{1}+s_{2}-i} \partial_{x_{s_{1}}} \partial_{x_{s_{2}}}+\sum_{s>0} y_{s} \partial_{x_{i-s}}+2 K \sum_{t>0} t \partial_{y_{t}} \partial_{x_{t+i}}, \\
& e_{-i, 2}=-\sum_{\substack{s_{1}, s_{2} \in \mathbb{Z} \backslash\{i\} \\
s_{1}+s_{2}=2 i}} \partial_{x_{s_{1}}} \partial_{x_{s_{2}}}={ }^{(\star)}-\sum_{s \in \mathbb{Z} \backslash\{i\}} \partial_{x_{2 i-s}} \partial_{x_{s}}
\end{aligned}
$$

$(\star): s_{1}+s_{2}=2 i \Longrightarrow s_{2}=2 i-s_{1}$.

Observemos que $e_{-i, 1}\left(x_{i}^{-p} Z\right)=x_{i}^{-p} e_{-i, 1} Z$, pois $e_{-i, 1}$ não depende de $x_{i}$. Pelo mesmo motivo, $x_{i} e_{-i, 2}\left(x_{i}^{-p} Z\right)=x_{i} x_{i}^{-p} e_{-i, 2} Z=x_{i}^{-p+1} e_{-i, 2} Z$.

Portanto, fazendo $Z^{\prime}=e_{-i, 1} Z, Z^{\prime \prime}=e_{-i, 2} Z$, para mostrar o que desejamos basta verificar que $e_{-i, 0}\left(x_{i}^{-p} Z\right)=A_{\alpha, p} x_{i}^{-p-1} Z$, já que

$$
e_{-i}\left(x_{i}^{-p} Z\right)=e_{-i, 0}\left(x_{i}^{-p} Z\right)+e_{-i, 1}\left(x_{i}^{-p} Z\right)+x_{i} e_{-i, 2}\left(x_{i}^{-p} Z\right)
$$

De fato,

$$
\begin{aligned}
e_{-i, 0}\left(x_{i}^{-p} Z\right) & =-x_{i} \partial_{x_{i}} \partial_{x_{i}}\left(x_{i}^{-p} Z\right)-2\left(\sum_{s \in \mathbb{Z} \backslash\{i\}} x_{s} \partial_{x_{s}}\right) \partial_{x_{i}}\left(x_{i}^{-p} Z\right)+(J-i K) \partial_{x_{i}}\left(x_{i}^{-p} Z\right) \\
& =-x_{i}(-p)(-p-1) x_{i}^{-p-2} Z+(J-i K)(-p) x_{i}^{-p-1} Z \\
& \left.-2\left(\sum_{s \in \mathbb{Z} \backslash\{i\}} x_{s} \partial_{x_{s}}\right)(-p) x_{i}^{-p-1} Z\right) \\
& =(-p)(p+1) x_{i}^{-p-1} Z+(J-i K)(-p) x_{i}^{-p-1} Z \\
& -2\left(\alpha_{1}(-p) x_{i}^{-p-1} Z+\ldots+\alpha_{k}(-p) x_{i}^{-p-1} Z\right) \\
& =\left(\left(-2 \sum_{j=1}^{k} \alpha_{j}\right)+p+1+J-i K\right)(-p) x_{i}^{-p-1} Z \\
& =(-p)(-2 \alpha+p+1+J-i K) x_{i}^{-p-1} Z=A_{\alpha, p} x_{i}^{-p-1} Z \in M_{i, S} .
\end{aligned}
$$

Para completar a prova, temos as seguintes observações:

(a) Se em $Z$ não houver um par $x_{i_{j}}, x_{i_{j^{\prime}}}, \operatorname{com} i_{j} \neq i, i_{j^{\prime}} \neq i$, e $i_{j}+i_{j^{\prime}}=2 i$, para $j, j^{\prime} \in\{1, \ldots, k\}$, então $Z^{\prime \prime}=e_{-i, 2} Z$ será o polinômio identicamente nulo;

(b) Se em $Z$ as três situações seguintes acontecem simultaneamente:

(i) Não há um par $x_{i_{j}}, x_{i_{j^{\prime}}}$, com $i_{j} \neq i, i_{j^{\prime}} \neq i$ e $i_{j}+i_{j^{\prime}} \neq 2 i$, para $j, j^{\prime} \in\{1, \ldots, k\}$; 
(ii) Não há $x_{i_{t}} \operatorname{com} i>i_{t}$, para $t \in\{1, \ldots, k\}$, e

(iii) Para $l \in\left\{k_{1}, \ldots, k_{n}, j_{1}, \ldots, j_{l}\right\}$ não há $x_{i_{t}}, t \in\{1, \ldots, k\}$ tal que $l+i=i_{t}$,

então $Z^{\prime}=e_{-i, 1} Z$ é o polinômio identicamente nulo.

Pelas observações acima, percebemos que quando $Z^{\prime}$ é o polinômio identicamente nulo, o mesmo não ocorre com $Z^{\prime \prime}$, e vice-versa. Porém, podem ser ambos não nulos, e é o caso que analisaremos em seguida.

Se ambos são diferentes do polinômio identicamente nulo, então são polinômios $d e g_{x^{+}}$-homogêneos, em que

$$
\operatorname{deg}_{x^{+}}\left(x_{i}^{-p+1} Z^{\prime \prime}\right)=1+(\alpha-2)=\alpha-1=\operatorname{deg}_{x^{+}}\left(x_{i}^{-p} Z^{\prime}\right)
$$

Em particular,

$$
\begin{aligned}
f_{i} e_{-i}\left(x_{i}^{-1} Z\right) & =f_{i}\left(A_{\alpha, 1} x_{i}^{-2} Z+x_{i}^{-1} Z^{\prime}+Z^{\prime \prime}\right)=x_{i}\left(A_{\alpha, 1} x_{i}^{-2} Z+x_{i}^{-1} Z^{\prime}+Z^{\prime \prime}\right) \\
& =A_{\alpha, 1} x_{i}^{-1} Z+Z^{\prime}+x_{i} Z^{\prime \prime}=A_{\alpha, 1} x_{i}^{-1} Z
\end{aligned}
$$

pois $Z^{\prime}$ e $x_{i} Z^{\prime \prime}$ são elementos de $I_{i, S}$.

Lema 5.4.14. Seja $J-i K \notin \mathbb{Z}$. Seja $v=x_{i}^{-1} h$, em que $h=h(\mathbf{x}, \mathbf{y})$ é um polinômio deg $x_{x^{+}}$ homogêneo tal que $\operatorname{deg}_{x^{+}} h=\alpha$ e $\partial_{x_{i}}(h)=0$. Então, existe $n>i$ tal que

$$
w=\left(e_{-i} f_{i}-A_{\alpha, 1}\right)\left(e_{-i} f_{i}-A_{\alpha+1,2}\right) e_{-n}(v)
$$

é não nulo em $M_{i, S}$ e $\operatorname{deg}_{x^{+}} w<\operatorname{deg}_{x^{+}} v$.

Demonstração. Em primeiro lugar, consideremos o caso em que $h(\mathbf{x}, \mathbf{y})=x_{i_{1}}^{\alpha_{1}} \ldots x_{i_{k}}^{\alpha_{k}} g(\mathbf{y})$, ou seja, $h$ é um monômio em $\mathbf{x}$ e um polinômio em $\mathbf{y}$. Seja $n>\max \left\{i, i_{1}, \ldots, i_{k}\right\}$. Vamos avaliar $e_{-n}(v)$ :

$$
\begin{aligned}
e_{-n}(v) & =e_{-n}\left(x_{i}^{-1} h\right)=-\sum_{m, k \in \mathbb{Z}} x_{k+m-n} \partial_{x_{k}} \partial_{x_{m}}\left(x_{i}^{-1} h\right)+\sum_{k>0} y_{k} \partial_{x_{-k+n}}\left(x_{i}^{-1} h\right) \\
& +2 K \sum_{m>0} m \partial_{y_{m}} \partial_{x_{m+n}}\left(x_{i}^{-1} h\right)+(J-n K) \partial_{x_{n}}\left(x_{i}^{-1} h\right) \\
& =-x_{2 i-n} \partial_{x_{i}} \partial_{x_{i}}\left(x_{i}^{-1} h\right)-2\left(\sum_{j=1}^{k} x_{i_{j}+i-n} \partial_{x_{i_{j}}}\right) \partial_{x_{i}}\left(x_{i}^{-1} h\right)-\sum_{j, t=1}^{k} x_{i_{j}+i_{t}-n} \partial_{x_{i_{j}}} \partial_{x_{i_{t}}}\left(x_{i}^{-1} h\right) \\
& +y_{n-i} \partial_{x_{i}}\left(x_{i}^{-1} h\right)+\sum_{j=1}^{k} y_{n-i_{j}} \partial_{x_{i_{j}}}\left(x_{i}^{-1} h\right),
\end{aligned}
$$

pois como $n>\max \left\{i, i_{1}, \ldots, i_{k}\right\}$, então as parcelas $\sum_{m>0} m \partial_{y_{m}} \partial_{x_{m+n}}\left(x_{i}^{-1} h\right)$ e $(J-n K) \partial_{x_{n}}\left(x_{i}^{-1} h\right)$ são nulas. Não é difícil perceber que as parcelas restantes são não nulas. Assim, 


$$
\begin{aligned}
e_{-n}\left(x_{i}^{-1} h\right) & =-2 x_{i}^{-3} x_{2 i-n} h-2\left(\sum_{j=1}^{k} x_{i_{j}+i-n} \partial_{x_{i_{j}}}\right)\left((-1) x_{i}^{-2} h\right)-\sum_{j, t=1}^{k} x_{i_{j}+i_{t}-n} x_{i}^{-1} \alpha_{t} \alpha_{j} x_{i_{j}}^{-1} x_{i_{t}}^{-1} h \\
& -y_{n-i} x_{i}^{-2} h+\sum_{j=1}^{k} y_{n-i_{j}} x_{i}^{-1} \alpha_{j} x_{i_{j}}^{-1} h \\
& =-2 x_{i}^{-3} x_{2 i-n} h+2 x_{i}^{-2}\left(\sum_{j=1}^{k} x_{i_{j}+i-n} \alpha_{j} x_{i_{j}}^{-1} h\right)-\sum_{j, t=1}^{k} x_{i_{j}+i_{t}-n} x_{i}^{-1} \alpha_{t} \alpha_{j} x_{i_{j}}^{-1} x_{i_{t}}^{-1} h \\
& -y_{n-i} x_{i}^{-2} h+\sum_{j=1}^{k} y_{n-i_{j}} x_{i}^{-1} \alpha_{j} x_{i_{j}}^{-1} h \\
& =-2 x_{i}^{-3} x_{2 i-n} h+x_{i}^{-2}\left(2 \sum_{j=1}^{k} \alpha_{j} x_{i+i_{j}-n} x_{i_{j}}^{-1} h-y_{n-i} h\right) \\
& +x_{i}^{-1}\left(\sum_{j=1}^{k} \alpha_{j} x_{i_{j}}^{-1} y_{n-i_{j}} h-\sum_{j, t=1}^{k} \alpha_{i_{j}, i_{t}} x_{i_{j}}^{-1} x_{i_{t}}^{-1} x_{i_{j}+i_{t}-n} h\right)
\end{aligned}
$$

em que $\alpha_{i_{j}, i_{t}}=\alpha_{i_{j}}\left(\alpha_{i_{t}}-\delta_{j, t}\right)$. Por simplicidade, seja

$$
\tilde{e}_{n}=\left(e_{-i} f_{i}-A_{\alpha, 1}\right)\left(e_{-i} f_{i}-A_{\alpha+1,2}\right) e_{-n}
$$

Vamos agora avaliar $\tilde{e}_{n}(v)$. Por facilidade, faremos os cálculos separadamente:

$$
\begin{aligned}
f_{i} e_{-n}(v) & =-2 x_{i}^{-2} x_{2 i-n} h+x_{i}^{-1}\left(2 \sum_{j=1}^{k} \alpha_{j} x_{i+i_{j}-n} x_{i_{j}}^{-1} h-y_{n-i} h\right) \\
& +\left(\sum_{j=1}^{k} \alpha_{j} x_{i_{j}}^{-1} y_{n-i_{j}} h-\sum_{j, t=1}^{k} \alpha_{i_{j}, i_{t}} x_{i_{j}}^{-1} x_{i_{t}}^{-1} x_{i_{j}+i_{t}-n} h\right) \\
& =-2 x_{i}^{-2} x_{2 i-n} h+x_{i}^{-1}\left(2 \sum_{j=1}^{k} \alpha_{j} x_{i+i_{j}-n} x_{i_{j}}^{-1} h-y_{n-i} h\right),
\end{aligned}
$$

pois $\left(\sum_{j=1}^{k} \alpha_{j} x_{i_{j}}^{-1} y_{n-i_{j}} h-\sum_{j, t=1}^{k} \alpha_{i_{j}, i_{t}} x_{i_{j}}^{-1} x_{i_{t}}^{-1} x_{i_{j}+i_{t}-n} h\right) \in I_{i, S}$.

Para calcular $e_{-i} f_{i} e_{-n}(v)$, utilizaremos que $e_{-i}=e_{-i, 0}+e_{-i, 1}+x_{i} e_{-i, 2}$, como definidos em (5.10). Assim, 


$$
\begin{aligned}
e_{-i} f_{i} e_{-n}(v) & =-2 A_{\alpha+1,2} x_{i}^{-3} x_{2 i-n} h-2 x_{i}^{-2} e_{-i, 1}\left(x_{2 i-n} h\right)-2 x_{i}^{-1} e_{-i, 2}\left(x_{2 i-n} h\right) \\
& +A_{\alpha, 1} x_{i}^{-2}\left(2 \sum_{j=1}^{k} \alpha_{j} x_{i+i_{j}-n} x_{i_{j}}^{-1} h-y_{n-i} h\right)+x_{i}^{-1} e_{-i, 1}\left(2 \sum_{j=1}^{k} \alpha_{j} x_{i+i_{j}-n} x_{i_{j}}^{-1} h-y_{n-i} h\right) \\
& +x_{i} x_{i}^{-1} e_{-i, 2}\left(2 \sum_{j=1}^{k} \alpha_{j} x_{i+i_{j}-n} x_{i_{j}}^{-1} h-y_{n-i} h\right) \\
& =-2 A_{\alpha+1,2} x_{i}^{-3} x_{2 i-n} h-2 x_{i}^{-2} e_{-i, 1}\left(x_{2 i-n} h\right)-2 x_{i}^{-1} e_{-i, 2}\left(x_{2 i-n} h\right) \\
& +A_{\alpha, 1} x_{i}^{-2}\left(2 \sum_{j=1}^{k} \alpha_{j} x_{i+i_{j}-n} x_{i_{j}}^{-1} h-y_{n-i} h\right)+x_{i}^{-1} e_{-i, 1}\left(2 \sum_{j=1}^{k} \alpha_{j} x_{i+i_{j}-n} x_{i_{j}}^{-1} h-y_{n-i} h\right),
\end{aligned}
$$

pois $x_{i} x_{i}^{-1} e_{-i, 2}\left(2 \sum_{j=1}^{k} \alpha_{j} x_{i+i_{j}-n} x_{i_{j}}^{-1} h-y_{n-i} h\right) \in I_{i, S}$.

Portanto,

$$
\begin{aligned}
\left(e_{-i} f_{i}-A_{\alpha+1,2}\right) e_{-n}(v) & =-2 x_{i}^{-2} e_{-i, 1}\left(x_{2 i-n} h\right)+A_{\alpha, 1} x_{i}^{-2}\left(2 \sum_{j=1}^{k} \alpha_{j} x_{i+i_{j}-n} x_{i_{j}}^{-1} h-y_{n-i} h\right) \\
& -A_{\alpha+1,2} x_{i}^{-2}\left(2 \sum_{j=1}^{k} \alpha_{j} x_{i+i_{j}-n} x_{i_{j}}^{-1} h-y_{n-i} h\right)-2 x_{i}^{-1} e_{-i, 2}\left(x_{2 i-n} h\right) \\
& +x_{i}^{-1} e_{-i, 1}\left(2 \sum_{j=1}^{k} \alpha_{j} x_{i+i_{j}-n} x_{i_{j}}^{-1} h-y_{n-i} h\right) \\
& -A_{\alpha+1,2} x_{i}^{-1}\left(\sum_{j=1}^{k} \alpha_{j} x_{i_{j}}^{-1} y_{n-i_{j}} h-\sum_{j, t=1}^{k} \alpha_{i_{j}, i_{t}} x_{i_{j}}^{-1} x_{i_{t}}^{-1} x_{i_{j}+i_{t}-n} h\right)
\end{aligned}
$$

Completaremos nos passos seguintes a análise de $\tilde{e}_{n}(v)$ :

$$
\begin{aligned}
f_{i}\left(e_{-i} f_{i}-A_{\alpha+1,2}\right) e_{-n}(v) & =-2 x_{i}^{-1} e_{-i, 1}\left(x_{2 i-n} h\right)+A_{\alpha, 1} x_{i}^{-1}\left(2 \sum_{j=1}^{k} \alpha_{j} x_{i+i_{j}-n} x_{i_{j}}^{-1} h-y_{n-i} h\right) \\
& -A_{\alpha+1,2} x_{i}^{-1}\left(2 \sum_{j=1}^{k} \alpha_{j} x_{i+i_{j}-n} x_{i_{j}}^{-1} h-y_{n-i} h\right),
\end{aligned}
$$

pois após a aplicação de $f_{i}$ (que age como multiplicação por $x_{i}$ ) o restante das parcelas está em $I_{i, S}$.

Novamente utilizaremos que $e_{-i}=e_{-i, 0}+e_{-i, 1}+x_{i} e_{-i, 2}$, como definidos em (5.10): 


$$
\begin{aligned}
e_{-i} f_{i}\left(e_{-i} f_{i}-A_{\alpha+1,2}\right) e_{-n}(v) & =-A_{\alpha, 1} 2 x_{i}^{-2} e_{-i, 1}\left(x_{2 i-n} h\right)-2 x_{i}^{-1} e_{-i, 1} e_{-i, 1}\left(x_{2 i-n} h\right) \\
& +A_{\alpha, 1} A_{\alpha, 1} x_{i}^{-2}\left(2 \sum_{j=1}^{k} \alpha_{j} x_{i+i_{j}-n} x_{i_{j}}^{-1} h-y_{n-i} h\right) \\
& +A_{\alpha, 1} x_{i}^{-1} e_{-i, 1}\left(2 \sum_{j=1}^{k} \alpha_{j} x_{i+i_{j}-n} x_{i_{j}}^{-1} h-y_{n-i} h\right) \\
& -A_{\alpha+1,2} A_{\alpha, 1} x_{i}^{-2}\left(2 \sum_{j=1}^{k} \alpha_{j} x_{i+i_{j}-n} x_{i_{j}}^{-1} h-y_{n-i} h\right) \\
& -A_{\alpha+1,2} x_{i}^{-1} e_{-i, 1}\left(2 \sum_{j=1}^{k} \alpha_{j} x_{i+i_{j}-n} x_{i_{j}}^{-1} h-y_{n-i} h\right),
\end{aligned}
$$

pois após aplicação de $x_{i} e_{-i, 2}$ o restante das parcelas está em $I_{i, S}$.

Portanto,

$$
\begin{aligned}
& w=\left(e_{-i} f_{i}-A_{\alpha, 1}\right)\left(e_{-i} f_{i}-A_{\alpha+1,2}\right) e_{-n}(v)=-2 x_{i}^{-1} e_{-i, 1} e_{-i, 1}\left(x_{2 i-n} h\right) \\
& -A_{\alpha+1,2} x_{i}^{-1} e_{-i, 1}\left(2 \sum_{j=1}^{k} \alpha_{j} x_{i+i_{j}-n} x_{i_{j}}^{-1} h-y_{n-i} h\right)+2 A_{\alpha, 1} x_{i}^{-1} e_{-i, 2}\left(x_{2 i-n} h\right) \\
& +A_{\alpha, 1} A_{\alpha+1,2} x_{i}^{-1}\left(\sum_{j=1}^{k} \alpha_{j} x_{i_{j}}^{-1} y_{n-i_{j}} h-\sum_{j, t=1}^{k} \alpha_{i_{j}, i_{t}} x_{i_{j}}^{-1} x_{i_{t}}^{-1} x_{i_{j}+i_{t}-n} h\right)
\end{aligned}
$$

e podemos perceber que $\operatorname{deg}_{x^{+}} w<\operatorname{de} g_{x^{+}} v$.

É importante destacar que há meios para controlarmos a escolha de $n$, de modo que não exista $y_{n-i_{j}}$ em $g(\mathbf{y})$, para nenhum $j \in\{1, \ldots, k\}$. Uma possibilidade é escolher $n>t+i_{j}$, para todo $t \in \mathbb{N}$ tal que $y_{t}$ está em $g(\mathbf{y})$, e todo $j \in\{1, \ldots, k\}$. Simultaneamente, a condição $n>\max \left\{i, i_{1}, \ldots, i_{k}\right\}$ continuará sendo exigida.

Em seguida, reescreveremos $w$ de forma mais conveniente para os nossos objetivos: para cada $j \in\{1, \ldots, k\}$, queremos apresentar $w$ como uma soma de dois polinômios, em que um deles possui $y_{n-i_{j}}$ e o outro não. As únicas parcelas que possuem relevância para nossa análise são aquelas em que haverá a aplicação de $e_{-i, 1}$, pois controlando a escolha de $n$ para que não haja $y_{n-i_{j}}$ em $g(\mathbf{y})$, para nenhum $j$, a única forma de produzi-los é com $e_{-i, 1}$. Novamente, dividiremos em casos.

Em primeiro lugar, analisaremos

$$
-A_{\alpha+1,2} x_{i}^{-1} e_{-i, 1}\left(2 \sum_{j=1}^{k} \alpha_{j} x_{i+i_{j}-n} x_{i_{j}}^{-1} h-y_{n-i} h\right),
$$

e o que de fato queremos avaliar é

$$
\sum_{s>0} y_{s} \partial_{x_{i-s}}\left(2 \sum_{j=1}^{k} \alpha_{j} x_{i+i_{j}-n} x_{i_{j}}^{-1} h-y_{n-i} h\right),
$$

pois é a única parcela que pode fornecer $y_{n-i_{j}}$, para $j \in\{1, \ldots, k\}$. Assim, para cada $j \in\{1, \ldots, k\}$, 
se $i-s=i+i_{j}-n$, então $s=n-i_{j}>0$. Logo,

$$
\begin{aligned}
& \sum_{s>0} y_{s} \partial_{x_{i-s}}\left(2 \sum_{j=1}^{k} \alpha_{j} x_{i+i_{j}-n} x_{i_{j}}^{-1} h-y_{n-i} h\right) \\
& =2 \sum_{j=1}^{k} \alpha_{j} x_{i_{j}}^{-1} y_{n-i_{j}} h+\sum_{\substack{s>0 \\
s \neq n-i_{j}}} y_{s} \partial_{x_{i-s}}\left(2 \sum_{j=1}^{k} \alpha_{j} x_{i+i_{j}-n} x_{i_{j}}^{-1} h-y_{n-i} h\right),
\end{aligned}
$$

o que nos permite separar $-A_{\alpha+1,2} x_{i}^{-1} e_{-i, 1}\left(2 \sum_{j=1}^{k} \alpha_{j} x_{i+i_{j}-n} x_{i_{j}}^{-1} h-y_{n-i} h\right)$ em uma soma do seguinte modo:para cada $j$,

$$
-2 A_{\alpha+1,2} x_{i}^{-1} \alpha_{j} x_{i_{j}}^{-1} y_{n-i_{j}} h+p
$$

em que $p$ é um polinômio com $\partial_{y_{n-i_{j}}}(p)=0$.

Avaliaremos em seguida $-2 x_{i}^{-1} e_{-i, 1} e_{-i, 1}\left(x_{2 i-n} h\right)$ :

$$
\begin{aligned}
& e_{-i, 1}\left(x_{2 i-n} h\right)=-2\left(\sum_{j=1}^{k} x_{2 i-n+i_{j}-i} \partial_{x_{i_{j}}}\right) \partial_{x_{2 i-n}}\left(x_{2 i-n} h\right) \\
& -\sum_{\substack{s_{1}, s_{2} \in \mathbb{Z} \backslash\{i\} \\
s_{1}+s_{2} \neq 2 i}} x_{2 i-n} x_{s_{1}+s_{2}-i} \partial_{x_{s_{1}}} \partial_{x_{s_{2}}}(h)+\sum_{s>0} y_{s} \partial_{x_{i-s}}\left(x_{2 i-n} h\right)+2 K \sum_{t>0} t \partial_{y_{t}} \partial_{x_{t+i}}\left(x_{2 i-n} h\right) \\
& =-2 \sum_{j=1}^{k} x_{2 i-n+i_{j}-i} \partial_{x_{i_{j}}}(h)-\sum_{\substack{s_{1}, s_{2} \in \mathbb{Z} \backslash\{i\} \\
s_{1}+s_{2} \neq 2 i}} x_{2 i-n} x_{s_{1}+s_{2}-i} \partial_{x_{s_{1}}} \partial_{x_{s_{2}}}(h)+y_{n-i} h+\sum_{\substack{s>0 \\
s \neq n-i}} y_{s} x_{2 i-n} \partial_{x_{i-s}}(h) \\
& +2 K \sum_{t>0} t \partial_{y_{t}} \partial_{x_{t+i}}\left(x_{2 i-n} h\right) .
\end{aligned}
$$

Observemos que foi usado o fato de que $2 i-n+i_{j} \neq 2 i$, já que $n>i_{j}$, para todo $j \in\{1, \ldots, k\}$, e a parcela $-2\left(\sum_{j=1}^{k} x_{2 i-n+i_{j}-i} \partial_{x_{i_{j}}}\right) \partial_{x_{2 i-n}}\left(x_{2 i-n} h\right)$ é não nula. A única parcela que poderia produzir $y_{n-i_{j}}$, para algum $j$, é $\sum_{s>0} y_{s} \partial_{x_{i-s}}\left(x_{2 i-n} h\right)$, o que não ocorre, pois como $n>i, h$ fornece apenas elementos da forma $y_{i-i_{j}}$ (quando fornece), e $x_{2 i-n}$ fornece $y_{n-i}$. Na verdade, para $h$, nunca serão produzidos novos $y_{n-i_{j}}$. Como aplicaremos $e_{-i, 1}$ novamente, estamos interessados nas parcelas em que foram produzidos novos $x_{m}, m \in \mathbb{Z}$, pois é apenas a partir deles que ainda podemos produzir $y_{n-i_{j}}$, para algum $j$. Verificaremos também o que ocorre com $x_{2 i-n}$. Para avaliar $e_{-i, 1} e_{-i,}\left(x_{2 i-n} h\right)$, estamos interessados apenas em $\sum_{s>0} y_{s} \partial_{x_{i-s}}\left(e_{-i, 1}\left(x_{2 i-n} h\right)\right)$, e assim, impondo mais algumas condições na escolha de $n$, a única parcela de fato interessante é

$$
\begin{aligned}
& \sum_{s>0} y_{s} \partial_{x_{i-s}}\left(-2 \sum_{j=1}^{k} x_{i+i_{j}-n} \partial_{x_{i_{j}}}(h)\right)=\sum_{s>0} y_{s} \partial_{x_{i-s}}\left(-2 \sum_{j=1}^{k} \alpha_{j} x_{i_{j}}^{-1} x_{i+j-n} h\right) \\
& =-2 \sum_{j=1}^{k} y_{n-i_{j}} \alpha_{j} x_{i_{j}}^{-1} h+\sum_{\substack{s>0 \\
s=i-i_{j}}} y_{s} \partial_{x_{i-s}}\left(-2 \sum_{j=1}^{k} \alpha_{j} x_{i_{j}}^{-1} x_{i+i_{j}-n} h\right) .
\end{aligned}
$$

Como $h$ não fornece $y_{n-i_{j}}$, para nenhum $j$, então a parcela importante é

$-2 \sum_{j=1}^{k} y_{n-i_{j}} \alpha_{j} x_{i_{j}}^{-1} h$ 
Portanto, $-2 x_{i}^{-1} e_{-i, 1} e_{-i, 1}\left(x_{2 i-n} h\right)$ é composto pela parcela $4 x_{i}^{-1} \sum_{j=1}^{k} y_{n-i_{j}} \alpha_{j} x_{i_{j}}^{-1} h$ somada a um polinômio $q$ no qual não temos $y_{n-i_{j}}$, para nenhum $j \in\{1, \ldots, k\}$.

Resumindo os cálculos que fizemos, podemos concluir que para cada $j=1, \ldots, k$ e $n$ suficientemente grande, temos o seguinte:

$$
w=\tilde{e}_{n} v=\alpha_{j} C_{\alpha} x_{i}^{-1} x_{i_{j}}^{-1} y_{n-i_{j}} h+g_{i_{j}},
$$

em que $C_{\alpha}=A_{\alpha, 1} A_{\alpha+1,2}-2 A_{\alpha+1,2}+4=2(2-2 \alpha+J-i K)(3-2 \alpha+J-i K)$ e $\partial_{y_{n-i_{j}}}\left(g_{i_{j}}\right)=0$.

É pertinente observar que, tendo em vista a possibilidade de controle na escolha de $n \in \mathbb{N}$, ele pode ser escolhido suficientemente grande de acordo com as necessidades. Se além das condições que já foram impostas, nós também impusermos $n>t+r$, para todo $r \neq i, r \neq i_{j}$, e $t \in \mathbb{N}$ tal que $y_{t}$ está em $g(\mathbf{y})$, então podemos garantir que $\partial_{y_{n-r}}\left(\tilde{e}_{n}\left(x_{i}^{-1} h\right)\right)=0$ se $r \neq i_{j}, j=1, \ldots k$, e $r \neq i$. Portanto, para todo $i^{\prime} \neq i$, temos as seguintes relações:

Se $i^{\prime} \neq i_{j}$, para todo $j=1, \ldots k$, então

$$
\partial_{y_{n-i^{\prime}}}\left(\tilde{e}_{n}\left(x_{i}^{-1} h\right)\right)=0 \text { e } \partial_{y_{n-i^{\prime}}}\left(C_{\alpha} x_{i}^{-1} y_{n-i^{\prime}} \partial_{x_{i^{\prime}}}(h)\right)=0
$$

Se $i^{\prime}=i_{j}$, para algum $j=1, \ldots, k$, então

$$
\begin{aligned}
\partial_{y_{n-i^{\prime}}}\left(\tilde{e}_{n}\left(x_{i}^{-1} h\right)\right) & =\partial_{y_{n-i_{j}}}\left(\tilde{e}_{n}\left(x_{i}^{-1} h\right)\right) \\
& =\partial_{y_{n-i_{j}}}\left(\alpha_{j} C_{\alpha} x_{i}^{-1} x_{i_{j}}^{-1} y_{n-i_{j}} h+g_{i_{j}}\right)=\partial_{y_{n-i_{j}}}\left(\alpha_{j} C_{\alpha} x_{i}^{-1} x_{i_{j}}^{-1} y_{n-i_{j}} h\right)+\partial_{y_{n-i_{j}}}\left(g_{i_{j}}\right) \\
& =\partial_{y_{n-i_{j}}}\left(C_{\alpha} x_{i}^{-1} y_{n-i_{j}} \partial_{x_{i_{j}}}(h)\right) .
\end{aligned}
$$

Concluímos então que para $i \neq i^{\prime}$,

$$
\partial_{y_{n-i^{\prime}}}\left(\tilde{e}_{n}\left(x_{i}^{-1} h\right)-C_{\alpha} x_{i}^{-1} y_{n-i^{\prime}} \partial_{x_{i^{\prime}}}(h)\right)=0 .
$$

Como $J-i K \notin \mathbb{Z}$, então $C_{\alpha} \neq 0$. Também $\partial_{x_{i_{j}}} h \neq 0$, para todo $j=1, \ldots, k$. Concluímos então que $w \neq 0$.

Consideremos agora um polinômio arbitrário $h(\mathbf{x}, \mathbf{y})$ e fixemos $i^{\prime}$ tal que $\partial_{x_{i^{\prime}}} h \neq 0$. Seja $h=$ $h_{1}+\ldots+h_{t}$, em que cada $h_{i}$ é um monômio em $\mathbf{x}$.

Aplicando (5.11) a cada $h_{s}$, e então tomando a soma sobre $s=1, \ldots, t$, temos

$$
w=\beta\left(C_{\alpha} x_{i}^{-1} y_{n-i^{\prime}} \partial_{x_{i^{\prime}}}(h)\right)+h^{\prime}
$$

$\operatorname{com} \beta \in \mathbb{N}$ a quantidade de monômios em $\mathbf{x}$ nos quais $x_{i^{\prime}}$ "aparece", e para algum $h^{\prime}$ tal que $\partial_{y_{n-i^{\prime}}}\left(h^{\prime}\right)=0$. Consequentemente, $w \neq 0$. A desigualdade $\operatorname{deg}_{x^{+}} w<\operatorname{deg}_{x^{+}} v$ segue do Lema 5.4.13.

Notemos que a hipótese de $d e g_{x^{+}}$-homogêneo para $h$ garante que $C_{\alpha}$ seja sempre o mesmo. 
Lema 5.4.15. Seja $v \in M_{i, S}$. Então, existe $u \in \mathfrak{U}$ tal que $u(v)$ é um elemento não nulo deg $x_{x^{-}}$ homogêneo em $M_{i, S}$.

Demonstração. Em primeiro lugar, notemos que $w \in M_{i, S}$ é $\operatorname{deg}_{x}$-homogêneo se e somente se $h_{0}(w)=\lambda w$, para algum $\lambda \in \mathbb{C}$, em que $h_{0}=-2 \sum_{m \in \mathbb{Z}} x_{m} \partial_{x_{m}}+J$. Escrevemos $v$ como uma soma $v=w_{1}+\ldots+w_{t}$ de polinômios homogêneos, e seja $h_{0}\left(w_{i}\right)=\lambda_{i} w_{i}$, em que $\lambda_{1}, \ldots, \lambda_{t}$ são dois a dois distintos. Então, para cada $k=0, \ldots, t-1$, temos

$$
h_{0}^{k}(v)=\lambda_{1}^{k} w_{1}+\ldots+\lambda_{t}^{k} w_{t}
$$

Teremos então um sistema da forma

$$
\begin{aligned}
h_{0}^{0}(v) & =h_{0}^{0}\left(w_{1}\right)+\ldots+h_{0}^{0}\left(w_{t}\right)=w_{1}+\ldots+w_{t} \\
h_{0}^{1}(v) & =h_{0}\left(w_{1}\right)+\ldots+h_{0}\left(w_{t}\right)=\lambda_{1} w_{1}+\ldots+\lambda_{t} w_{t} \\
\vdots & \\
h_{0}^{t-1}(v) & =h_{0}^{t-1}\left(w_{1}\right)+\ldots+h_{0}^{t-1}\left(w_{t}\right)=\lambda_{1}^{t-1} w_{1}+\ldots+\lambda_{t}^{t-1} w_{t} .
\end{aligned}
$$

Notemos que a matriz transposta da matriz dos coeficientes deste sistema é exatamente a matriz de Vandermonde, cujo determinante é dado por

$$
\prod_{1 \leq i<j \leq t}\left(\lambda_{j}-\lambda_{i}\right)
$$

e é não nulo, pois $\lambda_{j} \neq \lambda_{i}$, para todo $i \neq j$.

Portanto, o sistema possui única solução. Podemos assim encontrar $u_{i} \in \mathfrak{U}$ tal que $u_{i}(v)=w_{i}$, para todo $i$, e temos o resultado desejado.

Demonstraremos agora a Proposição 5.4.6(b)(ii):

Seja $v \in M_{i, S}$ não nulo qualquer. Se $v$ for um elemento da forma $x_{i}^{-1} g(\mathbf{x}, \mathbf{y})$, para algum polinômio $g$ tal que $\partial_{x_{i}} g=0$, estamos prontos para a demonstração. Caso não seja, podemos aplicar $f_{i}^{N}$, para algum $N>0$ suficientemente grande, e substitui-lo por $f_{i}^{N}(v)$. Além disso, usando o Lema 5.4.15, podemos assumir que $g$ é $d e g_{x^{-}}$homogêneo, de onde segue que é $d e g_{x^{+}}$-homogêneo. Podemos claramente perceber que $\operatorname{deg}_{x^{+}}(v)=\operatorname{deg}_{x^{+}}(g)$, e faremos indução em $\operatorname{deg}_{x^{+}}(v)$. O Lema 5.4.11 fornece o resultado desejado quando $\operatorname{deg}_{x}(v)=0$, e será nossa base de indução.

Suponhamos agora que $\operatorname{deg}_{x}(v)=\alpha \geq 1$. Então, fixamos $j \in \mathbb{Z}$ tal que $\partial_{x_{j}}(v) \neq 0$. Assim, pelo Lema 5.4.14, podemos encontrar $n>0$ suficientemente grande tal que $\tilde{e}_{n}(v) \neq 0$ em $M_{i, S}$, em que

$$
\tilde{e}_{n}=\left(e_{-i} f_{i}-A_{\alpha, 1}\right)\left(e_{-i} f_{i}-A_{\alpha+1,2}\right) e_{-n}
$$

Assumindo como hipótese de indução que o resultado seja válido sempre que $\operatorname{deg}_{x}(v)<\alpha$, 
em que $v$ é um polinômio não nulo $d e g_{x}$-homogêneo em $M_{i, S}$, teremos que $\tilde{e}_{n}(v)$ a satisfaz, pois $\operatorname{deg}_{x}\left(\tilde{e}_{n}(v)\right)<\operatorname{deg}_{x}(v)$. Assim, podemos encontrar $u_{1} \in \mathfrak{U}$ tal que $u_{1}\left(\tilde{e}_{n}(v)\right)=x_{i}^{-1} y_{k_{1}}^{-1} \ldots y_{k_{n}}^{-1}$. Consequentemente, $u=u_{1} \tilde{e}_{n} \in \mathfrak{U}$ fornece o resultado desejado.

\subsubsection{O Caso $K=0$}

Nesta seção nós demonstraremos as condições necessárias e suficientes para que $M_{i}$ seja irredutível, que é o item (b) do Teorema 5.3.3. Pelo Corolário 5.4.5, já temos que se $J \in \mathbb{Z}$, então $M_{i}$ é redutível. Assim, precisamos apenas analisar o que ocorre quando $J \notin \mathbb{Z}$. A próxima Proposição que provaremos será suficiente para o que desejamos.

Proposição 5.4.16. Seja $K=0$ e $J \notin \mathbb{Z}$. Então, temos o seguinte:

(a) $\mathfrak{U}\left(x_{i}^{-1}\right)=M_{i}$;

(b) Seja $v \in M_{i}$ não nulo. Então, existe $u \in \mathfrak{U}$ tal que $u(v)=x_{i}^{-1}$.

Começamos com o próximo Lema, que implica o item $(a)$ :

Lema 5.4.17. Seja $v \in M_{i}$ um elemento não nulo tal que deg $g_{x^{+}}(v)=0$. Então, existe $u_{1} \in \mathfrak{U}$ tal que $u_{1}(v)=x_{i}^{-1}$. Além disso, $\mathfrak{U}\left(x_{i}^{-1}\right)=M_{i}$.

Demonstração. Em primeiro lugar, fixamos $p \in \mathbb{N}$. Para $v=x_{i}^{-p}$, definimos $u_{1}=f_{i}^{p-1}$. No caso em que $v=\sum_{j=1}^{t} x_{i}^{-p_{j}}, p_{j} \in \mathbb{N}$, para todo $j=1, \ldots, t$, seja $p_{\max }=\max \left\{p_{j}, j=1, \ldots, t\right\}$. Para este $p_{\max }$ fixado, pela parte anterior, encontramos $u_{1} \in \mathfrak{U}$ tal que $u_{1}(v)=x_{i}^{-1}$, tendo em vista que $u_{1}(v)$ é um polinômio cujas parcelas ou são iguais a $x_{i}^{-1}$ ou estão em $I_{i}$.

Temos também o seguinte:

$$
\frac{-1}{p(p+1+J)} e_{-i}\left(x_{i}^{-p}\right)=x_{i}^{-p-1}
$$

De fato,

$$
\begin{aligned}
e_{-i}\left(x_{i}^{-p}\right) & =-x_{i} \partial_{x_{i}} \partial_{x_{i}}\left(x_{i}^{-p}\right)+J \partial_{x_{i}}\left(x_{i}^{-p}\right)=-x_{i}(-p)(-p-1) x_{i}^{-p-2}+J(-p) x_{i}^{-p-1} \\
& =(-p)(p+1) x_{i}^{-p-1}+J(-p) x_{i}^{-p-1}=(-p)(p+1+J) x_{i}^{-p-1}
\end{aligned}
$$

e temos então a igualdade desejada. Observamos que como $J \notin \mathbb{Z}$, então $p(p+1+J) \neq 0$

Podemos perceber facilmente que também vale a identidade

$$
\prod_{s=1}^{k} f_{i_{s}}^{\alpha_{s}}\left(x_{i}^{-p}\right)=x_{i}^{-p} x_{i_{1}}^{\alpha_{1}} \ldots x_{i_{n}}^{\alpha_{n}}
$$

para todo $p \in \mathbb{N}$. Para mostrar que $\mathfrak{U}\left(x_{i}^{-1}\right)=M_{i}$, como um polinômio em $M_{i}$ pode ser escrito como uma soma finita de monômios, precisamos apenas mostrar que cada monômio pode ser obtido de $x_{i}^{-1}$ através da ação de algum elemento $u \in \mathfrak{U}$.

Um monômio em $M_{i}$ é da forma $w=x_{i}^{-p} x_{i_{1}}^{\alpha_{j}} \ldots x_{i_{k}}^{\alpha_{k}}$. Assim, basta tomar 


$$
u=\left(\prod_{s=1}^{k} f_{i_{s}}^{\alpha_{s}}\right)\left(\frac{-1}{p(p+1+J)} e_{-i}\right)^{p-1}\left(x_{i}^{-1}\right)
$$

e teremos o resultado desejado.

Pela primeira parte do lema acima, já vimos que para um elemento $v$ de $M_{i}$ não nulo satisfazendo $\operatorname{deg}_{x^{+}}(v)=0$, existe $u \in \mathfrak{U}$ tal que $u(v)=x_{i}^{-1}$. Precisamos ainda mostrar que o resultado é válido quando $\operatorname{deg}_{x^{+}}(v)>0$, para concluir a demonstração da Proposição 5.4.16.

O item $(b)$ segue do Lema 5.4.15 (para $S=\emptyset$ ) e do seguinte Lema (pois a partir do momento que podemos diminuir $\operatorname{deg}_{x^{+}}(v)$, podemos obter $\left.x_{i}^{-1}\right)$ :

Lema 5.4.18. Seja $v \in M_{i}$ tal que $\operatorname{deg}_{x^{+}}(v)>0$. Então, existe $u \in \mathfrak{U} \operatorname{com} u(v)=v^{\prime} \neq 0$ e $\operatorname{deg}_{x^{+}}\left(v^{\prime}\right)<\operatorname{deg}_{x^{+}}(v)$.

Demonstração. Em primeiro lugar, assumiremos que $v$ é um monômio da forma $v=x_{i}^{-1} x_{i_{1}}^{\alpha_{1}} \ldots x_{i_{k}}^{\alpha_{k}}$. Caso não seja, podemos aplicar uma potência conveniente de $f_{i}$ a $v$. Para cada $s \in \mathbb{Z}, s \neq i$, consideremos

$$
e_{-s}(v)=-\sum_{m, k \in \mathbb{Z}} x_{k+m-s} \partial_{x_{k}} \partial_{x_{m}}(v)+J \partial_{x_{s}}(v)
$$

Por simplicidade, faremos os cálculos separadamente:

$$
\begin{aligned}
& -\sum_{m, k \in \mathbb{Z}} x_{k+m-s} \partial_{x_{k}} \partial_{x_{m}}(v)=-x_{2 i-s} \partial_{x_{i}} \partial_{x_{i}}(v)-2\left(\sum_{m \in \mathbb{Z} \backslash\{i\}} x_{m+i-s} \partial_{x_{m}}\right) \partial_{x_{i}}(v) \\
& -\left(\sum_{m_{1}, m_{2} \in \mathbb{Z} \backslash\{i\}} x_{m_{1}+m_{2}-s} \partial_{x_{m_{1}}} \partial_{x_{m_{2}}}\right)(v) \\
& =-x_{2 i-s}(-1)(-2) x_{i}^{-3} x_{i_{1}}^{\alpha_{1}} \ldots x_{i_{k}}^{\alpha_{k}}-2\left(\sum_{m \in \mathbb{Z} \backslash\{i\}} x_{m+i-s} \partial_{x_{m}}\right)\left((-1) x_{i_{1}}^{\alpha_{1}} \ldots x_{i_{k}}^{\alpha_{k}} x_{i}^{-2}\right) \\
& -\left(\sum_{m_{1}, m_{2}=1}^{k} \alpha_{m_{1}} \alpha_{m_{2}} x_{-s+i_{m_{1}}+i_{m_{2}}} x_{i}^{-1} x_{i_{m_{1}}}^{-1} x_{i_{m_{2}}}^{-1} x_{i_{1}}^{\alpha_{1}} \ldots x_{i_{k}}^{\alpha_{k}}\right) \\
& =-2 x_{i}^{-3} x_{i_{1}}^{\alpha_{1}} \ldots x_{i_{k}}^{\alpha_{k}} x_{2 i-s}+2 x_{i}^{-2}\left(\sum_{m=1}^{k} x_{m+i-s} \alpha_{m} x_{i_{m}}^{-1} x_{i_{1}}^{\alpha_{1}} \ldots x_{i_{k}}^{\alpha_{k}}\right) \\
& -x_{i}^{-1}\left(\sum_{m_{1}, m_{2}=1}^{k} \alpha_{m_{1}}\left(\alpha_{m_{2}}-\delta_{m_{1}, m_{2}}\right) x_{-s+i_{m_{1}}+i_{m_{2}}} x_{i_{m_{1}}}^{-1} x_{i_{m_{2}}}^{-1} x_{i_{1}}^{\alpha_{1}} \ldots x_{i_{k}}^{\alpha_{k}}\right)=v_{s} . \\
& J \partial_{x_{s}}(v)=\sum_{j=1}^{k} \delta_{s, i_{j}} J \alpha_{j} x_{s}^{-1} v .
\end{aligned}
$$

Portanto, podemos escrever $e_{-s}(v)=v_{s}+\sum_{j=1}^{k} \delta_{s, i_{j}} \alpha_{j} J x_{s}^{-1} v$.

Seguindo o raciocínio da demonstração do Lema 5.4.14, definimos 


$$
\tilde{e}_{s}=\left(e_{-i} f_{i}-A_{\alpha, 1}\right)\left(e_{-i} f_{i}-A_{\alpha+1,2}\right) e_{-s}
$$

Seja também

$$
\tilde{v}_{s}=\left(e_{-i} f_{i}-A_{\alpha, 1}\right)\left(e_{-i} f_{i}-A_{\alpha+1,2}\right)\left(v_{s}\right)
$$

Suponhamos agora que $\tilde{e}_{s}(v)=0$, para todo número inteiro $s$ tal que $s \notin\left\{i_{1}, \ldots, i_{k}\right\}$. Já sabemos que, para $s$ nestas condições $\sum_{j=1}^{k} \delta_{s, i_{j}} J \alpha_{j} x_{s}^{-1} v=0$. Portanto, $\tilde{v}_{s}=0$, para todos esses $s$. Observemos porém, que isto só ocorre se $v_{s}=0$, para todos esses $s$. Como os coeficientes de $v_{s}$ não dependem de $s$, então $v_{s}=0$, para todo $s \in \mathbb{Z}$. Em particular, para $s=i_{1}, v_{i_{1}}=0$.

Assim,

$$
\begin{aligned}
\tilde{e}_{i_{1}}(v)=\left(e_{-i} f_{i}-A_{\alpha, 1}\right)\left(e_{-i} f_{i}-A_{\alpha+1,2}\right)\left(\alpha_{1} J x_{i_{1}}^{-1} v\right) & =e_{-i} f_{i} e_{-i} f_{i}\left(\alpha_{1} J x_{i_{1}}^{-1} v\right) \\
& -e_{-i} f_{i} A_{\alpha+1,2}\left(\alpha_{1} J x_{i_{1}}^{-1} v\right)-A_{\alpha, 1} e_{-i} f_{i}\left(\alpha_{1} J x_{i_{1}}^{-1} v\right) \\
& +A_{\alpha, 1} A_{\alpha+1,2} \alpha_{1} J x_{i_{1}}^{-1} v=A_{\alpha, 1} A_{\alpha+1,2} \alpha_{1} J x_{i_{1}}^{-1} v,
\end{aligned}
$$

pois ao aplicarmos $f_{i}$, as outras parcelas estarão em $I_{i}$, e $e_{-i}$ não produz potências negativas de $x_{i}$. Como $A_{\alpha, 1}$ e $A_{\alpha+1,2}$ são não nulos, já que $J \notin \mathbb{Z}$, então $\tilde{e}_{i_{1}}(v)$ é não nulo; e também

$$
\operatorname{deg}_{x^{+}}\left(\tilde{e}_{i_{1}}(v)\right)=\alpha-1<\alpha=\operatorname{deg}_{x^{+}}(v),
$$

o que nos dá o resultado desejado quando $v$ é um monômio.

Consideremos agora $v$ um polinômio arbitrário. Então, $v$ pode ser escrito como

$$
v=v_{1}+\ldots+v_{t}
$$

com $v_{1}, \ldots, v_{t}$ linearmente independentes. Tendo em vista que podemos aplicar uma potência conveniente de $f_{i}$ a $v$ se necessário, vamos assumir que todos os monômios têm $x_{i}$-grau igual a -1 . Observemos então, que cada monômio é da forma

$$
v_{m}=x_{i}^{-1} x_{i_{1 m}}^{\alpha_{1 m}} \ldots x_{i_{k_{m}}}^{\alpha_{k m}}
$$

Repetindo os passos que fizemos para um monômio, consideremos $s \in \mathbb{Z}$ tal que $s \neq i$. Suponhamos que $\tilde{e}_{s}(v)=0$, para todo $s \notin\left\{i_{1 m}, \ldots, i_{k m} \mid m=1, \ldots, t\right\}$. Como $v_{s}=\left(v_{1}\right)_{s}+\ldots+\left(v_{t}\right)_{s}$ tem coeficientes que não dependem de $s$, então podemos fixar $s \in\left\{i_{1 m}, \ldots, i_{k m} \mid m=1, \ldots, t\right\}$, que denotaremos por $i_{1}$, por exemplo. Assim,

$$
\tilde{e}_{i_{1}}(v)=A_{\alpha, 1} A_{\alpha+1,2} J x_{i_{1}}^{-1}\left(\alpha_{1}\left(v_{1}\right) v_{1}+\ldots+\alpha_{1}\left(v_{t}\right) v_{t}\right),
$$


em que $\alpha_{j}\left(v_{j}\right)$ é o grau de $x_{i_{1}}$ em $v_{j}$. Como os monômios são linearmente independentes, e $A_{\alpha, 1} A_{\alpha+1,2} J \neq 0$, e temos o resultado desejado. 


\section{Apêndice A}

\section{Notação, Definições e Resultados Básicos}

Este apêndice tem por objetivo apresentar os conceitos fundamentais para o estudo das álgebras de Lie e suas representações, bem como fixar notação e enunciar os principais resultados. Alguns deles serão apenas enunciados, com a indicação da bibliografia onde as demonstrações podem ser encontradas sem maior dificuldade. Apresentaremos também alguns resultados para anéis e módulos, que sejam importantes no desenvolvimento do trabalho, mas mantendo o foco principal nas álgebras de Lie.

Destacamos ainda que as álgebras de Kac-Moody, em particular as álgebras de Lie afim, são tratadas em capítulo específico.

\section{A.1 Anéis e Módulos}

Nesta seção, $A$ será um anel associativo, não necessariamente comutativo, com elemento identidade $1 \neq 0$. Quando não especificarmos se $A$ é comutativo ou não, o resultado enunciado será válido para ambos os casos. Denotaremos o produto de dois elementos $a_{1}, a_{2} \in A$ por $a_{1} a_{2}$; da mesma forma, para $a \in A$ e $m \in M$, em que $M$ é um $A$-módulo à esquerda, a ação de $A$ em $M$ será denotada por $a m$.

O que faremos aqui será especificamente utilizado no desenvolvimento do Capítulo 4.

As principais referências utilizadas foram [11], [10] e [1].

Definição A.1.1. Seja A um anel. Um conjunto multiplicativo (de forma mais completa, um conjunto multiplicativamente fechado unital) em $A$ é um subconjunto $S \subseteq A$ tal que:

(a) $1 \in S, e$

(b) Se $s_{1}, s_{2} \in S$, então $s_{1} s_{2} \in S$ (isto é, $S$ é fechado sob a multiplicação de A).

Definição A.1.2 (Condição de Ore). Sejam $A$ um anel e $S$ um conjunto multiplicativo em $A$. Diremos que $S$ satisfaz a condição de Ore à esquerda quando, para cada $s \in S$ e a $\in$ A, existem $t \in S$ e $b \in A$ tais que $t a=b s$, isto é, $S a \cap A s \neq \emptyset$. Um conjunto multiplicativo satisfazendo a condição de Ore à esquerda é chamado abreviadamente de conjunto de Ore à esquerda. Podemos definir simetricamente condição de Ore à direita e conjunto de Ore à direita.

Um conjunto de Ore é um conjunto multiplicativo que satisfaz as condições de Ore à esquerda e à direita simultaneamente. 
Notemos que, em anéis comutativos, qualquer conjunto multiplicativo é um conjunto de Ore.

Lema A.1.3. Sejam A um anel, e $S$ um conjunto de Ore à esquerda em A. Dados quaisquer elementos $s_{1}, \ldots, s_{n} \in S$, existem $a_{1}, \ldots, a_{n} \in A$ tais que $a_{1} s_{1}=\ldots=a_{n} s_{n}$ e $a_{1} s_{1} \in S$, isto é, $A s_{1} \cap \ldots \cap A s_{n} \cap S \neq \emptyset$.

Demonstração. A demonstração é feita por indução em $n$ :

- Base de Indução $(n=2)$ : sejam $s_{1}, s_{2} \in S$; existem $a_{1} \in S$ e $a_{2} \in A$ tais que $a_{1} s_{1}=a_{2} s_{2}$, e $a_{1} s_{1} \in S$, pois $S$ é um conjunto de Ore à esquerda.

- Hipótese de Indução: suponhamos agora que o resultado seja válido para $n=k>2$, isto é, dados $s_{1}, s_{2}, \ldots, s_{k} \in S$, existem $b_{1}, b_{2}, \ldots, b_{k} \in A$ tais que $b_{1} s_{1}=b_{2} s_{2}=\ldots=b_{k} s_{k}$ e $b_{1} s_{1} \in S$.

- Seja $s_{k+1} \in S$. Como $S$ é um conjunto de Ore à esquerda, existem $b_{k+1} \in A$ e $b_{1}^{\prime} \in S$ tais que $b_{1}^{\prime}\left(b_{1} s_{1}\right)=b_{k+1} s_{k+1}$. Temos também que $b_{1}^{\prime}\left(b_{1} s_{1}\right) \in S$, pois S é um conjunto multiplicativo, e pela hipótese de indução $b_{1} s_{1} \in S$.

Assim, $b_{1}^{\prime}\left(b_{1} s_{1}\right)=b_{1}^{\prime}\left(b_{2} s_{2}\right)=\ldots=b_{1}^{\prime}\left(b_{k} s_{k}\right)=b_{k+1} s_{k+1}$ e $b_{1}^{\prime}\left(b_{1} s_{1}\right) \in S$, o que conclui a demonstração.

Lema A.1.4. Sejam $A$ um anel, e $S$ um conjunto de Ore à esquerda em $A$. Suponha que $\phi: A \longrightarrow B$ seja um homomorfismo de anéis tal que $\phi(s)$ é uma unidade de $B$, para todo $s \in S$. Então, dados $a_{1}, \ldots, a_{n} \in A$ e $s_{1}, \ldots, s_{n} \in S$, existem $x_{1}, \ldots, x_{n} \in A$ e $y \in S$ tais que $\phi\left(s_{i}\right)^{-1} \phi\left(a_{i}\right)=\phi(y)^{-1} \phi\left(x_{i}\right)$, para todo $i=1, \ldots, n$.

Demonstração. Pelo Lema A.1.3, existem $b_{1}, \ldots, b_{n} \in A$ e $y \in S$ tais que $b_{i} s_{i}=y$, para todo $i=1, \ldots, n$. Então,

$$
\phi(y)=\phi\left(b_{i} s_{i}\right)=\phi\left(b_{i}\right) \phi\left(s_{i}\right) \Longrightarrow \phi(y) \phi\left(s_{i}\right)^{-1}=\phi\left(b_{i}\right),
$$

isto é, $\phi\left(b_{i}\right)$ é uma unidade em $B$. Então, se $x_{i}=b_{i} a_{i}$, para todo $i=1, \ldots, n$, temos

$$
\phi(y)^{-1} \phi\left(x_{i}\right)=\phi\left(b_{i} s_{i}\right)^{-1} \phi\left(b_{i} a_{i}\right)=\phi\left(s_{i}\right)^{-1} \phi\left(b_{i}\right)^{-1} \phi\left(b_{i}\right) \phi\left(a_{i}\right)=\phi\left(s_{i}\right)^{-1} \phi\left(a_{i}\right) .
$$

Notemos que, usando a simetria da definição, os dois lemas anteriores podem ser enunciados e demonstrados também para um conjunto de Ore à direita.

A partir do Lema A.1.4, ao estudarmos anéis de frações no Capítulo 4, teremos condições de afirmar que as "frações" $\phi\left(s_{i}\right)^{-1} \phi\left(a_{i}\right), i=1, \ldots, n$, possuem um "denominador comum" $\phi(y)$.

Definição A.1.5 (Restrição de Escalares). ([1, p.27]) Sejam A e $B$ anéis comutativos, $M$ um $B$-módulo, e $f: A \longrightarrow B$ um homomorfismo de anéis. Então, $M$ possui estrutura de A-módulo definida como segue: se $a \in A$ e $m \in M$, então am $:=f(a) m$. Dizemos que este A-módulo é obtido de $M$ por restrição de escalares. Em particular, $f$ define uma estrutura de A-módulo em $B$. 
De modo análogo, definimos restrição de escalares para anéis não comutativos, especificando se $M$ é um $A$-módulo à esquerda ou à direita.

Definição A.1.6 (Extensão de Escalares). ([1, p.28]) Sejam $A$ e $B$ anéis comutativos, $M$ um A-módulo, e $f: A \longrightarrow B$ um homomorfismo de anéis; pela Definição A.1.5, B tem estrutura de $A$-módulo, e podemos então obter o $A$-módulo $M_{B}=B \otimes_{A} M . M_{B}$ tem também uma estrutura de $B$-módulo tal que $b\left(b^{\prime} \otimes m\right)=\left(b b^{\prime}\right) \otimes m$, para todo $b, b^{\prime} \in B$ e $m \in M$, e dizemos que foi obtido de $M$ por extensão de escalares.

É fácil ver que a Definição A.1.5 faz sentido, pois $\operatorname{Im} f \subseteq B$.

A Definição A.1.6, porém, necessita de demonstração, o que optamos por não fazer aqui, apenas enunciando (como encontrado em [1]), tendo em vista que um resultado análogo para módulos sobre anéis não comutativos é demonstrado no Capítulo 2.

O conceito de funtor é também bastante importante para o Capítulo 4, mas para que possamos defini-lo, precisamos antes passar pelo conceito de categoria.

Definição A.1.7. ([11, p.9-10]) Uma categoria $\mathcal{C}$ consiste de:

(a) Uma classe ob $\mathcal{C}$ de objetos (geralmente denotados por $A, B, C$,etc.);

(b) Para cada par ordenado de objetos $(A, B)$, um conjunto hom $\cos _{\mathcal{C}}(A, B)$, cujos elementos são chamados morfismos de $A$ em $B$. Ao sabermos exatamente a qual categoria nos referimos, escrevemos simplesmente hom $(A, B)$;

(c) Para cada tripla ordenada de objetos $(A, B, C)$, uma aplicação $(f, g) \longmapsto g f$ de $\operatorname{hom}(A, B) \times \operatorname{hom}(B, C)$ em hom $(A, C)$.

É necessário que os objetos e morfismos satisfaçam as seguintes condições:

(i) $S e(A, B) \neq(C, D)$, então hom $(A, B)$ e hom $(C, D)$ são disjuntos;

(ii) (Associatividade) Se $f \in h o m(A, B), g \in h o m(B, C)$ e $h \in h o m(C, D)$, então

$$
(h g) f=h(g f) .
$$

De forma simplificada, escrevemos hgf;

(iii) (Unidade) Para cada objeto $A$, temos um elemento $1_{A} \in$ hom $(A, A)$ tal que $f 1_{A}=f$, para todo $f \in \operatorname{hom}(A, B)$ e $1_{A} g=g$ para cada $g \in \operatorname{hom}(B, A)\left(1_{A}\right.$ é único).

Exemplo A.1.8. $A$-mod, a categoria dos módulos à esquerda sobre um anel $A$ fixado; ob $A$-mod é a classe dos módulos à esquerda de $A$ e os morfismos são os homomorfismos de $A$-módulos à esquerda; o produto de homomorfismos é a composição deles.

Definição A.1.9. ([11, p.19]) Sejam $\mathcal{C}$ e $\mathcal{D}$ categorias. Um funtor (covariante) $F$ de $\mathcal{C}$ em $\mathcal{D}$ consiste de:

(a) Uma aplicação $A \longmapsto F A$ de ob $\mathcal{C}$ em ob $\mathcal{D}$; 
(b) Para cada par de objetos $(A, B)$ de $\mathcal{C}$, uma aplicação

$$
f \longmapsto F(f)
$$

$\operatorname{de~hom}_{\mathcal{C}}(A, B) e m \operatorname{hom}_{\mathcal{D}}(F A, F B)$.

É necessário que satisfaçam as seguintes condições:

(i) Se gf está definida em $\mathcal{C}$, então $F(g f)=F(g) F(f), e$

(ii) $F\left(1_{A}\right)=1_{F A}$.

\section{A.2 Álgebras de Lie}

Nesta seção apresentamos algumas definições e resultados básicos para as álgebras de Lie, que podem ser encontrados principalmente em [4] e [15]. Salvo menção em contrário, todos os espaços vetoriais serão sobre um corpo $\mathbb{K}$ arbitrário.

Os resultados mais específicos são apresentados nos capítulos onde se fazem necessários.

Definição A.2.1. Uma álgebra de Lie consiste de um espaço vetorial $\mathfrak{g}$ munido de um produto (colchete ou comutador)

$$
[,]: \mathfrak{g} \times \mathfrak{g} \longrightarrow \mathfrak{g}
$$

com as seguintes propriedades:

(a) É bilinear;

(b) Antissimétrico, isto é, $[X, X]=0$ para todo $X \in \mathfrak{g}$ (o que implica $[X, Y]=-[Y, X]$ para todo $X, Y \in \mathfrak{g}$ e é equivalente se o corpo de escalares não é de característica dois), $e$

(c) Satisfaz a identidade de Jacobi, isto é, para todo $X, Y, Z \in \mathfrak{g}$,

$$
[X,[Y, Z]]+[Z,[X, Y]]+[Y,[Z, X]]=0 .
$$

Esta igualdade por ser reescrita alternativamente de umas das duas formas:

(c1) $[X,[Y, Z]]=[[X, Y], Z]+[Y,[X, Z]]$,

(c2) $[[X, Y], Z]=[[X, Z], Y]+[X,[Y, Z]]$.

É conveniente observar que uma álgebra de Lie $\mathfrak{g}$ não é necessariamente associativa, pois $[[X, X], Y]=0$ para quaisquer $X, Y \in \mathfrak{g}$, mas $[X,[X, Y]]$ nem sempre se anula.

Definição A.2.2. Seja $\mathfrak{g}$ uma álgebra de Lie. Uma subálgebra de $\mathfrak{g}$ é um subespaço vetorial $\mathfrak{h}$ de $\mathfrak{g}$ que é fechado pelo colchete, isto é, $[X, Y] \in \mathfrak{h}$ se $X, Y \in \mathfrak{h}$.

Notemos que uma subálgebra de Lie é uma álgebra de Lie com a estrutura herdada de $\mathfrak{g}$. 
Exemplo A.2.3. $\mathfrak{g l}(n, \mathbb{K})$ : o espaço de todas as transformações lineares de um espaço vetorial de dimensão $n$ sobre o corpo $\mathbb{K}$, que é o mesmo que o espaço das matrizes $n \times n$ com coeficientes em $\mathbb{K}$. O colchete é dado por

$$
[X, Y]=X Y-Y X
$$

com $X$ e $Y$ matrizes. Algumas vezes, quando o corpo não for relevante, indicaremos essas álgebras apenas por $\mathfrak{g l}(n)$. Da mesma forma, indicaremos a álgebra das transformações lineares de um espaço vetorial $V$ qualquer (incluindo aqui espaços de dimensão infinita) por $\mathfrak{g l}(V)$. É importante observarmos que, na notação para espaços vetoriais, o espaço vetorial das transformações lineares de $V$ é denotado por $\operatorname{End}(V)$. Aqui, trata-se ainda do mesmo espaço vetorial, que ao ser dotado com a estrutura de álgebra de Lie recebe a denominação de $\mathfrak{g l}(V)$.

Notemos que o espaço de todas as transformações lineares de qualquer espaço vetorial $V$, de dimensão finita ou não, é uma álgebra associativa com o produto de duas transformações dado pela composição das mesmas. Assim, podemos generalizar o exemplo acima, como segue:

Exemplo A.2.4. (Álgebras de Lie Provenientes de Álgebras Associativas): Seja $\mathcal{A}$ uma álgebra associativa e em $\mathcal{A}$ defina o colchete pelo comutador

$$
[x, y]=x y-y x, \text { para todo } x, y \in \mathcal{A}
$$

Esse colchete define em $\mathcal{A}$ uma estrutura de álgebra de Lie, que alguns autores denotam por $[\mathcal{A}]$.

Exemplo A.2.5. (Subálgebras de $\mathfrak{g l}(n, \mathbb{K}))$ :

(a) $\mathfrak{s l}(n, \mathbb{K})=\{X \in \mathfrak{g l}(n, \mathbb{K}) \mid \operatorname{tr}(X)=0\}$. Muitas vezes essas álgebras são denotadas apenas por $\mathfrak{s l}(n)$;

(b) $\mathfrak{s o}(n, \mathbb{K})=\left\{X \in \mathfrak{g l}(n, \mathbb{K}) \mid X+X^{t}=0\right\}$, em que $X^{t}$ indica a matriz transposta da matriz $X$;

Notemos que o espaço das matrizes simétricas

$$
\left\{X \in \mathfrak{g l}(n, \mathbb{K}) \mid X=X^{t}\right\}
$$

não é subálgebra de $\mathfrak{g l}(n, \mathbb{K})$ se $n \geq 2$, pois se $X$ e $Y$ são simétricas, então $[X, Y]$ é antissimétrica. De fato, se $X, Y \in \mathfrak{g l}(n, \mathbb{K})$ são tais que $X=X^{t}$ e $Y=Y^{t}$, então

$$
\begin{aligned}
{[X, Y] } & =X Y-Y X \\
& =X^{t} Y^{t}-Y^{t} X^{t}=(Y X)^{t}-(X Y)^{t}=(Y X-X Y)^{t}=-(X Y-Y X)^{t}=-[X, Y]^{t}
\end{aligned}
$$

(c) O subespaço das matrizes triangulares superiores

$$
\left\{X \in \mathfrak{g l}(n, \mathbb{K}) \mid X=\left(\begin{array}{cccc}
a_{1} & * & \cdots & * \\
0 & a_{2} & \cdots & * \\
\vdots & \vdots & \ddots & \vdots \\
0 & 0 & \cdots & a_{n}
\end{array}\right)\right\}
$$


é uma subálgebra de $\mathfrak{g l}(n, \mathbb{K})$;

(d) $\mathfrak{s p}(n, \mathbb{K})=\left\{X \in \mathfrak{g l}(2 n, \mathbb{K}) \mid X J+J X^{t}=0\right\}$, em que $J$ é escrito em blocos $n \times n$ como

$$
J=\left(\begin{array}{cc}
0 & -1 \\
1 & 0
\end{array}\right),
$$

com 0 representando a matriz nula e 1 a matriz identidade $n \times n$. Para ver que esse subespaço é de fato uma subálgebra, notemos em primeiro lugar que $J^{2}=-1$ e, portanto, $X \in \mathfrak{s p}(n, \mathbb{K})$ se e somente se

$$
\begin{aligned}
J X^{t} & =-X J \\
J J X^{t} & =-J X J \\
X^{t} & =J X J .
\end{aligned}
$$

Se $X, Y \in \mathfrak{s p}(n, \mathbb{K})$, então

$$
\begin{aligned}
{[X, Y]^{t} } & =(X Y-Y X)^{t}=(X Y)^{t}-(Y X)^{t} \\
& =-X^{t} Y^{t}+Y^{t} X^{t} \\
& =-J X J^{2} Y J+J Y J^{2} X J \\
& =J(X Y-Y X) J \\
& =J[X, Y] J
\end{aligned}
$$

isto é, $[X, Y] \in \mathfrak{s p}(n, \mathbb{K})$.

Definição A.2.6. Diremos que uma álgebra de Lie $\mathfrak{g}$ é abeliana quando $[X, Y]=0$, para todo $X, Y \in \mathfrak{g}$. Neste caso, a estrutura de álgebra de Lie não acrescenta nada à estrutura de espaço vetorial.

Exemplo A.2.7. (a) Se $\operatorname{dim} \mathfrak{g}=1$, então $\mathfrak{g}$ é abeliana;

(b) Todo subespaço de dimensão 1 de uma álgebra de Lie qualquer é uma subálgebra abeliana;

(c) O espaço das matrizes diagonais é uma subálgebra abeliana de $\mathfrak{g l}(n, \mathbb{K})$.

Todo subespaço de uma álgebra abeliana é uma subálgebra.

\section{Observação A.2.8. (Álgebras de Dimensão $\leq 2$ )}

(a) $\operatorname{dim} \mathfrak{g}=1$, então $\mathfrak{g}$ é abeliana;

(b) $\operatorname{dim} \mathfrak{g}=2$, existem duas possibilidades:

(i) g é abeliana ou

(ii) Existe uma base $\{X, Y\}$ de $\mathfrak{g}$ tal que $[X, Y]=Y$ e a partir daí, o colchete de quaisquer 
dois elementos de $\mathfrak{g}$ é dado por

$$
[a X+b Y, c X+d Y]=(a d-b c)[X, Y]=(a d-b c) Y, \text { com } a, b, c, d \in \mathbb{K}
$$

De fato, suponhamos que $\mathfrak{g}$ não seja abeliana, e tomemos uma base $\left\{X^{\prime}, Y^{\prime}\right\}$ de $\mathfrak{g}$. Então, $\left[X^{\prime}, Y^{\prime}\right] \neq 0$, pois caso contrário $\mathfrak{g}$ seria abeliana. Seja $Y^{\prime \prime}=\left[X^{\prime}, Y^{\prime}\right]$ e escolha $X^{\prime \prime}$ tal que $\left\{X^{\prime \prime}, Y^{\prime \prime}\right\}$ seja base de $\mathfrak{g}$. Então, $X^{\prime \prime}=a X^{\prime}+b Y^{\prime}, Y^{\prime \prime}=c X^{\prime}+d Y^{\prime}$, com $a, b, c, d \in \mathbb{K}$, e $\left[X^{\prime \prime}, Y^{\prime \prime}\right]=\alpha Y^{\prime \prime}, \alpha \in \mathbb{K}$ com $\alpha \neq 0$ (pois $\left\{X^{\prime \prime}, Y^{\prime \prime}\right\}$ é base, e se $\alpha=0$, teríamos $\mathfrak{g}$ abeliana). Os elementos $X=\frac{1}{\alpha} X^{\prime \prime}$ e $Y=Y^{\prime \prime}$ formam a base desejada. Para tanto, basta notar que $\left\{\frac{1}{\alpha} X^{\prime \prime}, Y^{\prime \prime}\right\}$ é um conjunto linearmente independente.

Definição A.2.9. Uma transformação linear $\psi: \mathfrak{g} \longrightarrow \mathfrak{h}$ (com $\mathfrak{g}$ e h álgebras de Lie) é um

(a) Homomorfismo se $\psi([X, Y])=[\psi(X), \psi(Y)]$;

(b) Isomorfismo se for um homomorfismo inversivel;

(c) Automorfismo se é um isomorfismo e $\mathfrak{g}=\mathfrak{h}$.

As álgebras $\mathfrak{g}$ e $\mathfrak{h}$ são isomorfas se existe um isomorfismo $\psi: \mathfrak{g} \longrightarrow \mathfrak{h}$.

Exemplo A.2.10. (a) Os homomorfismos entre as álgebras abelianas são as transformações lineares. Logo, duas álgebras abelianas de dimensão finita são isomorfas se e só se elas têm a mesma dimensão;

(b) Se $\psi: \mathfrak{g} \longrightarrow \mathfrak{h}$ é um homomorfismo e $\mathfrak{h}$ é abeliana, então Ker $\psi$ contém todos os elementos da forma $[X, Y], X, Y \in \mathfrak{g}$, pois $\psi([X, Y])=[\psi(X), \psi(Y)]=0$;

(c) A aplicação traço

$$
\operatorname{tr}: \mathfrak{g l}(n, \mathbb{K}) \longrightarrow \mathbb{K}
$$

é um homomorfismo, pois $\operatorname{tr}(X Y-Y X)=0$ para quaisquer transformações lineares $X, Y$ e, portanto, $\operatorname{tr}([X, Y])=0=[\operatorname{tr}(X), \operatorname{tr}(Y)]$, já que $\mathbb{K}$, por ser de dimensão 1, é uma álgebra abeliana.

Definição A.2.11. Seja $\mathfrak{g}$ uma álgebra de Lie de dimensão finita com base $\left\{X_{1}, \ldots, X_{n}\right\}$. Tomando dois elementos $X_{i}, X_{j}$ desta base, o colchete entre eles $\left[X_{i}, X_{j}\right]$ pode ser escrito como uma combinação linear

$$
\left[X_{i}, X_{j}\right]=\sum_{k=1}^{n} c_{i j}^{k} X_{k}
$$

Os coeficientes $c_{i j}^{k} \in \mathbb{K}$ são denominados constantes de estrutura da álgebra em relação a uma base.

Conforme [15, p.22], é possível mostrar que duas álgebras de Lie de dimensão finita (ambas com a mesma dimensão) que possuem as mesmas constantes de estrutura são isomorfas. Assim, podemos 
afirmar que existem apenas duas álgebras de Lie de dimensão dois, a menos de isomorfismo: uma delas é abeliana, e a outra é a que admite uma base $\{X, Y\}$ tal que $[X, Y]=Y$.

Definição A.2.12. Um subespaço i de $\mathfrak{g}$ é um ideal de $\mathfrak{g} s e$

$$
\begin{array}{r}
\forall Y \in \mathfrak{i}, X \in \mathfrak{g},[X, Y] \in \mathfrak{i}, \text { isto é, } \\
{[\mathfrak{g}, \mathfrak{i}]=\operatorname{ger}\{[X, Y]: X \in \mathfrak{g}, Y \in \mathfrak{i}\} \subseteq \mathfrak{i} .}
\end{array}
$$

Notemos que, como o colchete de Lie é antissimétrico, todos os ideais são bilaterais.

Observação A.2.13. Todo ideal é claramente uma subálgebra. Notemos que, no entanto, nem toda subálgebra é um ideal. De fato, consideremos o subespaço de $\mathfrak{s l}(2, \mathbb{R})$ gerado por $\left(\begin{array}{cc}1 & 0 \\ 0 & -1\end{array}\right)$. Esse espaço é uma subálgebra de $\mathfrak{s l}(2, \mathbb{R})$, pois é unidimensional. Porém, não é um ideal, já que

$$
\left[\left(\begin{array}{cc}
1 & 0 \\
0 & -1
\end{array}\right),\left(\begin{array}{ll}
0 & 1 \\
0 & 0
\end{array}\right)\right]=\left(\begin{array}{ll}
0 & 2 \\
0 & 0
\end{array}\right) .
$$

Notemos também que todo subespaço de uma álgebra abeliana é um ideal.

Com as definições que temos até aqui, se $\psi: \mathfrak{g} \longrightarrow \mathfrak{h}$ for um homomorfismo de álgebras de Lie, então Ker $\psi$ é um ideal de $\mathfrak{g}$ e $\operatorname{Im} \psi$ é uma subálgebra de $\mathfrak{h}$.

Definição A.2.14. Seja $\mathfrak{g}$ uma álgebra de Lie e $\mathfrak{h}$ um ideal. No espaço vetorial quociente $\mathfrak{g} / \mathfrak{h}$, defina

$$
[\bar{X}, \bar{Y}]=\overline{[X, Y]}
$$

em que $\bar{X}$ denota a classe $X+\mathfrak{h}$, para todo $X \in \mathfrak{g}$. Esse colchete define em $\mathfrak{g} / \mathfrak{h}$ uma estrutura de álgebra de Lie.

(vide $[4$, p.3])

Definição A.2.15. De modo análogo ao que temos para anéis, a projeção canônica

$$
\begin{aligned}
\pi: \mathfrak{g} & \longrightarrow \mathfrak{g} / \mathfrak{h} \\
X & \longmapsto \bar{X}
\end{aligned}
$$

é um homomorfismo sobrejetor de álgebras de Lie.

Teorema A.2.16 (Teorema Fundamental do Isomorfismo). Seja $\psi: \mathfrak{g} \longrightarrow \mathfrak{h}$ um homomorfismo. Então,

$$
\mathfrak{g} / \operatorname{Ker} \psi \cong \operatorname{Im} \psi
$$

Para a demonstração, o procedimento é análogo ao utilizado para a demonstração do Teorema Fundamental do Isomorfismo para anéis. 
Exemplo A.2.17. Sejam

$$
\begin{aligned}
& \mathfrak{g}=\left\{X \in \mathfrak{g l}(3, \mathbb{K}) \mid X=\left(\begin{array}{ccc}
0 & * & * \\
0 & 0 & * \\
0 & 0 & 0
\end{array}\right)\right\} \mathrm{e} \\
& \mathfrak{h}=\left\{X \in \mathfrak{g l}(3, \mathbb{K}) \mid X=\left(\begin{array}{lll}
0 & 0 & * \\
0 & 0 & 0 \\
0 & 0 & 0
\end{array}\right)\right\} .
\end{aligned}
$$

$\mathfrak{h}$ é ideal de $\mathfrak{g}$, pois para todo $X \in \mathfrak{h}, Y \in \mathfrak{g},[X, Y]=0$. O quociente $\mathfrak{g} / \mathfrak{h}$ é uma álgebra abeliana bidimensional, pois dados $X, Y \in \mathfrak{g},[X, Y] \in \mathfrak{h}$, o que nos dá $[\bar{X}, \bar{Y}]=\overline{[X, Y]}=\overline{0}$.

\section{A.2.1 Representações de Álgebras de Lie}

A teoria de representações de álgebras de Lie tem um papel fundamental no estudo das mesmas, pois através de uma representação podemos descrever uma álgebra de Lie como uma álgebra de transformações lineares, o que algumas vezes pode facilitar bastante o trabalho.

Nesta subseção veremos apenas os resultados principais a respeito do assunto, e maiores detalhes podem ser encontrados em [4] e [15].

Definição A.2.18. Sejam $V$ um espaço vetorial, e $\mathfrak{g l}(V)$ a álgebra de Lie das transformações lineares de V. Seja também $\mathfrak{g}$ uma álgebra de Lie (sobre o mesmo corpo de escalares de $V$ ). Uma representação de $\mathfrak{g}$ em $V$ é um homomorfismo

$$
\rho: \mathfrak{g} \longrightarrow \mathfrak{g l}(V)
$$

Na terminologia usual, $V$ se denomina o espaço da representação, enquanto sua dimensão é a dimensão da representação. Uma representação $\rho$ é dita fiel se Ker $\rho=\{0\}$.

Notemos que, sempre que uma representação for fiel, pelo Teorema A.2.16, podemos olhar para $\mathfrak{g}$ como uma subálgebra da álgebra de Lie das transformações lineares de $V$.

Exemplo A.2.19. (a) Se $\mathfrak{g} \subseteq \mathfrak{g l}(V)$ é subálgebra, a inclusão define, de modo trivial, uma representação de $\mathfrak{g}$ em $V$, denominada representação canônica;

(b) A aplicação

$$
\left(\begin{array}{cc}
a & b \\
c & -a
\end{array}\right) \in \mathfrak{s l}(2, \mathbb{K}) \longmapsto\left(\begin{array}{ccc}
2 a & -2 b & 0 \\
-c & 0 & b \\
0 & 2 c & -2 a
\end{array}\right) \in \mathfrak{g l}(3, \mathbb{K})
$$

é uma representação de $\mathfrak{s l}(2, \mathbb{K})$. De fato, seja a base $\{X, H, Y\}$ de $\mathfrak{s l}(2, \mathbb{K})$, em que

$$
X=\left(\begin{array}{ll}
0 & 1 \\
0 & 0
\end{array}\right) \quad H=\left(\begin{array}{cc}
1 & 0 \\
0 & -1
\end{array}\right) \quad Y=\left(\begin{array}{ll}
0 & 0 \\
1 & 0
\end{array}\right) .
$$


Suas constantes de estrutura são dadas por

$$
[H, X]=2 X \quad[H, Y]=-2 Y \quad[X, Y]=H
$$

As imagens dos elementos dessa base formam uma base de $\operatorname{Im} \rho$, que tem as mesmas constantes de estrutura.

Definição A.2.20. Um módulo à esquerda sobre uma álgebra de Lie $\mathfrak{g}$ é um espaço vetorial $V$ sobre o mesmo corpo de escalares de $\mathfrak{g}$, juntamente com uma lei de composição externa $\mathfrak{g} \times V \longrightarrow V$, denotada por $(X, v) \longmapsto X v$, que satisfaz, para $X, Y \in \mathfrak{g}, u, v \in V$ e um escalar $x$, as seguintes propriedades:

(a) $(X+Y) v=X v+Y v$;

(b) $X(u+v)=X u+X v$;

(c) $(x X) v=X(x v)$;

(d) $[X, Y] v=X Y v-Y X v$.

Notemos que, simetricamente, podemos definir um módulo à direita. Porém, neste texto, sem perda de generalidade, ao nos referirmos aos módulos sobre uma álgebra de Lie, estaremos tratando de módulos à esquerda.

Observação A.2.21. Em um módulo $V$, cada $X \in \mathfrak{g}$ define uma aplicação linear de $V$ por multiplicação à esquerda:

$$
v \in V \longmapsto X v \in V .
$$

Em virtude das propriedades de módulo, essas aplicações lineares definem uma representação de $\mathfrak{g}$ em $V$. Vice versa, dada uma representação $\rho$ de $\mathfrak{g}$ em $V$, o produto $\mathfrak{g} \times V \longrightarrow V$ dado por

$$
(X, v) \longmapsto X v=\rho(X)(v)
$$

define um módulo sobre $\mathfrak{g}$. Em outras palavras, os conceitos de módulo e de representação são equivalentes.

Definição A.2.22. Para um elemento $X$ na álgebra de Lie $\mathfrak{g}$, considere a transformação linear

$$
\operatorname{ad}(X): \mathfrak{g} \longrightarrow \mathfrak{g}
$$

definida por ad $(X)(Y)=[X, Y]$. A aplicação

$$
a d: X \in \mathfrak{g} \longmapsto a d(X) \in \mathfrak{g l}(\mathfrak{g})
$$

define uma representação de $\mathfrak{g}$ em $\mathfrak{g}$, denominada representação adjunta. O fato de ad ser linear provém da bilinearidade do colchete. Já a propriedade de homomorfismo de ad é equivalente à 
identidade de Jacobi. De fato, a igualdade ad $([X, Y])=\operatorname{ad}(X) \operatorname{ad}(Y)-\operatorname{ad}(Y) \operatorname{ad}(X)$ é a mesma que

$$
[[X, Y], Z]=[[X, Z], Y]+[X,[Y, Z]], \text { para todo } Z \in \mathfrak{g}
$$

Definição A.2.23. O núcleo da representação adjunta é denominado centro de $\mathfrak{g}$ e é denotado $\operatorname{por} \mathfrak{z}(\mathfrak{g})$ :

$$
\mathfrak{z}(\mathfrak{g})=\{X \in \mathfrak{g} \mid \operatorname{ad}(X)(Y)=[X, Y]=0, \text { para todo } Y \in \mathfrak{g}\},
$$

isto é, o centro de uma álgebra de Lie é o conjunto de seus elementos que comutam com todos os seus elementos. Notemos que $\mathfrak{z}(\mathfrak{g})$ é um ideal abeliano de $\mathfrak{g}$.

Podemos generalizar a definição acima para qualquer subconjunto $A \subseteq \mathfrak{g}$, como segue:

Definição A.2.24. O centralizador de um subconjunto $A \subseteq \mathfrak{g}$ é definido como

$$
\mathfrak{z}(A)=\{Y \in \mathfrak{g} \mid \forall X \in A,[X, Y]=0\}
$$

Se $A=\mathfrak{g}$, as definições de centro e centralizador coincidem. O centralizador de qualquer $A \subseteq \mathfrak{g}$ é uma subálgebra de $\mathfrak{g}$, pois se $X, Y \in \mathfrak{z}(A)$ e $Z \in A$, então

$$
[[X, Y], Z]=[[X, Z], Y]+[X,[Y, Z]]=0
$$

já que $[X, Z]=[Y, Z]=0$.

Definição A.2.25. Uma representação $\rho$ de uma álgebra de Lie $\mathfrak{g}$ em um espaço vetorial $V$ é dita irredutivel se os únicos subespaços invariantes por $\rho$ são $V$ e $\{0\}$, o que é equivalente a dizer que

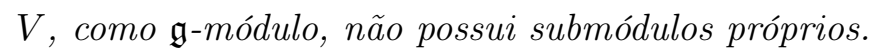

Definição A.2.26. Uma representação $\rho$ de uma álgebra de Lie $\mathfrak{g}$ em um espaço vetorial $V$ é dita completamente redutivel se $V$ se decompõe como uma soma direta de subespaços invariantes tais que a restrição de $\rho$ a cada um deles é irredutivel, isto é, como g-módulo, $V$ é uma soma direta de $\mathfrak{g}$-submódulos irredutiveis. As representações completamente redutíveis são também denominadas representações semissimples.

Notemos que uma representação irredutível é sempre completamente redutível.

Definição A.2.27. Uma aplicação linear $D: \mathfrak{g} \longrightarrow \mathfrak{g}$ é uma derivação da álgebra de Lie $\mathfrak{g}$ se satisfaz

$$
D([X, Y])=[D(X), Y]+[X, D(Y)], \text { para todo } X, Y \in \mathfrak{g} \text {. }
$$

Um exemplo de derivação que surge com frequência são as adjuntas dos elementos de $\mathfrak{g}$. Utilizando a identidade de Jacobi, para todo $Z \in \mathfrak{g}$, temos

$$
\begin{aligned}
& \operatorname{ad}(X)([Y, Z])=[X,[Y, Z]]=[[X, Y], Z]+[Y,[X, Z]] \text { isto é, } \\
& \operatorname{ad}(X)([Y, Z])=[\operatorname{ad}(X)(Y), Z]+[Y, \operatorname{ad}(X)(Z)]
\end{aligned}
$$

o que nos mostra que ad $(X)$ é de fato uma derivação. 
Definição A.2.28 (Série Derivada). Seja $\mathfrak{g}$ uma álgebra de Lie. Para dois subconjuntos $A$ e $B$ quaisquer de $\mathfrak{g}$, denotaremos por $[A, B]$ o subespaço de $\mathfrak{g}$

$$
[A, B]=<\{[X, Y] \mid X \in A, Y \in B\}>
$$

Definimos então, por indução, os seguintes subespaços de $\mathfrak{g}$ :

$$
\begin{aligned}
\mathfrak{g}^{(0)} & =\mathfrak{g} \\
\mathfrak{g}^{\prime} & =[\mathfrak{g}, \mathfrak{g}] \\
\vdots & \\
\mathfrak{g}^{(k)} & =\left[\mathfrak{g}^{(k-1)}, \mathfrak{g}^{(k-1)}\right] .
\end{aligned}
$$

Esses subespaços são ideais de $\mathfrak{g}$, pois para quaisquer ideais $\mathfrak{i}, \mathfrak{j}$ de $\mathfrak{g}$, a identidade de Jacobi implica que $[\mathfrak{i}, \mathbf{j}]$ é um ideal de $\mathfrak{g}$.

Essa sequência de ideais é conhecida como série derivada de $\mathfrak{g}$ e suas componentes são as álgebras derivadas de $\mathfrak{g}$.

Exemplo A.2.29. Seja $\mathfrak{g}=\mathfrak{s l}(2, \mathbb{R})$. Então, $\mathfrak{g}=\mathfrak{g}^{\prime}$ e, portanto, $\mathfrak{g}^{(k)}=\mathfrak{g}$, para todo $k \geq 0$. De fato, sejam

$$
X=\left(\begin{array}{ll}
0 & 1 \\
0 & 0
\end{array}\right) H=\left(\begin{array}{cc}
1 & 0 \\
0 & -1
\end{array}\right) Y=\left(\begin{array}{ll}
0 & 0 \\
1 & 0
\end{array}\right) .
$$

Então, $[H, X]=2 X,[H, Y]=-2 Y$ e $[X, Y]=H$ e, portanto, $X, Y$ e $H$ estão em $\mathfrak{g}^{\prime}$. Como $\{X, H, Y\}$ é uma base de $\mathfrak{g}$, então $\mathfrak{g}^{\prime}=\mathfrak{g}$. A mesma afirmação vale para $\mathfrak{s l}(2, \mathbb{K})$, desde que $\mathbb{K}$ seja um corpo de característica diferente de 2 .

Se a característica é $2, \mathfrak{g}^{\prime}$ é o subespaço de $\mathfrak{g}$ gerado por $H$ e, portanto, $\mathfrak{g}^{\prime \prime}=\{0\}$.

Definição A.2.30 (Álgebras Solúveis). Uma álgebra de Lie $\mathfrak{g}$ é solúvel se alguma de suas álgebras derivadas se anula, isto é,

$$
\mathfrak{g}^{\left(k_{0}\right)}=\{0\},
$$

para algum $k_{0} \geq 1$ (e, portanto, $\mathfrak{g}^{(k)}=\{0\}$ para todo $\left.k \geq k_{0}\right)$.

Exemplo A.2.31. Um exemplo clássico de álgebra solúvel são as álgebras de Lie das matrizes triangulares superiores sobre um corpo $\mathbb{K}$ :

$$
\mathfrak{g}=\left\{\left(\begin{array}{cccc}
* & * & \cdots & * \\
0 & * & \cdots & * \\
\vdots & \vdots & \ddots & \vdots \\
0 & 0 & \cdots & *
\end{array}\right)_{n \times n}\right\}
$$

em que $\mathfrak{g}^{(n)}=\{0\}$.

Proposição A.2.32. Seja $\mathfrak{g}$ uma álgebra de Lie. Então, 
(a) Se $\mathfrak{g}$ é solúvel, $e \mathfrak{h} \subseteq \mathfrak{g}$ é subálgebra, então $\mathfrak{h}$ também é solúvel (em particular, um ideal de uma álgebra solúvel é também solúvel);

(b) Se $\mathfrak{g}$ é solúvel $e \mathfrak{h} \subseteq \mathfrak{g}$ é um ideal, então $\mathfrak{g} / \mathfrak{h}$ também é solúvel.

(vide $[15, \mathrm{p} .46]$ )

A partir do resultado acima, podemos afirmar que imagens homomórficas de álgebras solúveis são também solúveis. De fato, se $\varphi: \mathfrak{g} \longrightarrow \mathfrak{h}$ é um homomorfismo sobrejetor de álgebras de Lie, pelo Teorema A.2.16, $\mathfrak{g} / \operatorname{Ker} \varphi \cong \mathfrak{h}$ e, pela proposição acima, $\mathfrak{g} / \operatorname{Ker} \varphi$ é solúvel, já que $\operatorname{Ker} \varphi$ é um ideal de $\mathfrak{g}$.

Proposição A.2.33. Sejam $\mathfrak{g}$ uma álgebra de Lie $e \mathfrak{h} \subseteq \mathfrak{g}$ um ideal. Suponha que tanto $\mathfrak{h}$ quanto $\mathfrak{g} / \mathfrak{h}$ sejam solúveis. Então, $\mathfrak{g}$ é solúvel.

(vide $[15, \mathrm{p} .47]$ )

Definição A.2.34 (Série Central Descendente). Seja $\mathfrak{g}$ uma álgebra de Lie. Definimos, por indução, a série central descendente como

$$
\begin{aligned}
\mathfrak{g}^{1} & =\mathfrak{g} \\
\mathfrak{g}^{2} & =[\mathfrak{g}, \mathfrak{g}]=\mathfrak{g}^{\prime} \\
& \vdots \\
\mathfrak{g}^{k} & =\left[\mathfrak{g}, \mathfrak{g}^{k-1}\right] .
\end{aligned}
$$

Como o produto de ideais é ideal, segue que $\mathfrak{g}^{k}$ é um ideal de $\mathfrak{g}$, para todo $k \geq 1$. Assim, temos $\mathfrak{g}^{k+1}=\left[\mathfrak{g}, \mathfrak{g}^{k}\right] \subseteq \mathfrak{g}^{k}$, o que nos dá uma cadeia descendente de ideais

$$
\mathfrak{g}^{1}=g \supseteq \mathfrak{g}^{2} \supseteq \ldots \supseteq \mathfrak{g}^{k} \supseteq \ldots
$$

Definição A.2.35. Seja $\mathfrak{g}$ uma álgebra de Lie. Diremos que $\mathfrak{g}$ é nilpotente se sua série central descendente se anula em algum momento, isto é, $\mathfrak{g}^{k_{0}}=\{0\}$, para algum $k_{0} \geq 1$ (e, portanto, $\mathfrak{g}^{k}=\{0\}$, para todo $\left.k \geq k_{0}\right)$.

Proposição A.2.36. Seja $\mathfrak{g}$ uma álgebra de Lie nilpotente.

(a) Se $\mathfrak{h} \subseteq \mathfrak{g}$ é subálgebra, então $\mathfrak{h}$ é nilpotente;

(b) Se $\mathfrak{h} \subseteq \mathfrak{g}$ é um ideal, então $\mathfrak{g} / \mathfrak{h}$ é nilpotente.

(vide $[15, \mathrm{p} .48]$ )

Proposição A.2.37. Seja $\mathfrak{g}$ uma álgebra de Lie. Então, sua série derivada tem decrescimento mais rápido do que sua série central descendente, isto é,

$$
\mathfrak{g}^{(k)} \subseteq \mathfrak{g}^{k+1},
$$

para todo $k \geq 0$. 
(vide [15, p.45])

Notemos que a proposição acima nos permite concluir que álgebras nilpotentes são solúveis.

A afirmação contrária porém, não é sempre verdadeira. Para obter um exemplo disso, basta observarmos que a álgebra de Lie das matrizes triangulares superiores é solúvel, mas não nilpotente.

Um outro exemplo de álgebra solúvel que não é nilpotente é a álgebra de dimensão dois não abeliana. Para essa álgebra, $\mathfrak{g}^{k} \neq\{0\}$, se $k \geq 2$, mas $\mathfrak{g}^{\prime \prime}=\{0\}$.

Exemplo A.2.38. O centro de uma álgebra de Lie nilpotente não é trivial. De fato, existe $k \in \mathbb{N}$ tal que $\mathfrak{g}^{k} \neq\{0\}$ e $\mathfrak{g}^{k+1}=\{0\}$. Assim, $\left[\mathfrak{g}, \mathfrak{g}^{k}\right]=\mathfrak{g}^{k+1}=\{0\}$ e, portanto, $\mathfrak{g}^{k} \subseteq \mathfrak{z}(\mathfrak{g})$.

No entanto, o centro de uma álgebra solúvel pode se anular; é o que ocorre, por exemplo, com a álgebra não abeliana de dimensão dois.

Proposição A.2.39. Seja $\mathfrak{g}$ uma álgebra de Lie de dimensão finita. Então, existe em $\mathfrak{g}$ um único ideal solúvel $\tau \subseteq \mathfrak{g}$ que contém todos os ideais solúveis de $\mathfrak{g}$. Esse ideal é chamado de radical solúvel de $\mathfrak{g}$, e usamos a notação $\tau(\mathfrak{g})$.

(vide [15, p.49])

$\mathrm{Na}$ verdade, o resultado é mais geral, pois é possível prová-lo também para álgebras de Lie de dimensão infinita.

Definição A.2.40. Uma álgebra de Lie $\mathfrak{g}$ é semissimples se não contém ideais solúveis além do ideal trivial $\{0\}$.

Definição A.2.41. Uma álgebra de Lie $\mathfrak{g}$ é simples se

(a) Os únicos ideais de $\mathfrak{g}$ são $\{0\}$ e $\mathfrak{g}, e$

(b) $\operatorname{dim} \mathfrak{g} \neq 1$.

Exemplo A.2.42. $\mathfrak{s l}(2, \mathbb{R})$ é simples. De fato, consideremos uma base formada pelos elementos

$$
X=\left(\begin{array}{ll}
0 & 1 \\
0 & 0
\end{array}\right) H=\left(\begin{array}{cc}
1 & 0 \\
0 & -1
\end{array}\right) Y=\left(\begin{array}{ll}
0 & 0 \\
1 & 0
\end{array}\right) .
$$

Os colchetes entre eles são dados por $[H, X]=2 X ;[H, Y]=-2 Y$ e $[X, Y]=H$. Suponha que exista um ideal $I$ de $\mathfrak{s l}(2, \mathbb{R})$ tal que $I \neq\{0\}$ e considere $Z=a X+b H+c Y \in I$, com $a, b, c \in \mathbb{R}$ e pelo menos um deles não nulo. Então,

$$
a d(X)(Z)=-2 b X+c H \text { e } a d^{2}(X)(Z)=-2 c X
$$

de onde temos que $Z, a d(X)(Z)$ ou $a d^{2}(X)(Z)$ é múltiplo não nulo de $X$, isto é, $X \in I$. Como $H=-[Y, X]$ e $I$ é ideal, então $H \in I$; e como $Y=1 / 2[Y, H]$, temos que $Y \in I$, pelo mesmo motivo. Assim, $I=\mathfrak{s l}(2, \mathbb{R})$, e $\mathfrak{s l}(2, \mathbb{R})$ é simples.

Observação A.2.43. Conforme Teorema 4.25 em [4], temos que $\mathfrak{s l}(n, \mathbb{C})$ é simples, para qualquer $n \in \mathbb{N}$. Na verdade, $\mathfrak{s l}(n, \mathbb{K})$ é simples sempre que $\mathbb{K}$ for um corpo de característica diferente de dois. 
Observação A.2.44. O centro de uma álgebra de Lie é um ideal abeliano e, portanto, solúvel como álgebra de Lie. Assim, o centro de uma álgebra semissimples é trivial.

Observação A.2.45. Como o centro de uma álgebra de Lie $\mathfrak{g}$ semissimples é trivial, então a representação adjunta dessa álgebra é uma representação fiel, pois o centro de uma álgebra de Lie é o núcleo de sua representação adjunta. Assim, podemos "olhar"para uma álgebra de Lie semissimples como uma subálgebra de $\mathfrak{g l}(\mathfrak{g})$.

Definição A.2.46. Sejam $\mathfrak{g}$ uma álgebra de Lie, e h uma subálgebra. O normalizador de $\mathfrak{h} e ́$ definido como

$$
\mathfrak{n}(\mathfrak{h})=\{X \in \mathfrak{g} \mid[X, \mathfrak{h}] \subseteq \mathfrak{h}\} .
$$

$\mathfrak{n}(\mathfrak{h})$ é uma subálgebra de $\mathfrak{g}$. De fato, sejam $X, Y \in \mathfrak{n}(\mathfrak{h}), H \in \mathfrak{h}$. Então,

$$
[[X, Y], H]=[[X, H], Y]+[X,[Y, H]],
$$

pela Identidade de Jacobi. Como $[X, H] \in \mathfrak{h} e[Y, H] \in \mathfrak{h}$, temos que $[[X, Y], H] \in \mathfrak{h}$, o que nos dá o resultado desejado.

Definição A.2.47. ([18, p.635]) Seja $\mathfrak{g}$ uma álgebra de Lie. Uma subálgebra $\mathfrak{h}$ de $\mathfrak{g}$ é chamada de subálgebra de Cartan de $\mathfrak{g}$ se:

(a) Todo elemento $h \in \mathfrak{h}$ é localmente nilpotente em $\mathfrak{h}$, isto é, fixando $h \in \mathfrak{h}$, para todo $h^{\prime} \in \mathfrak{h}$, existe $m^{\prime} \in \mathbb{N}$ tal que $a d^{m^{\prime}}(h)\left(h^{\prime}\right)=0$;

(b) h é seu próprio normalizador em $\mathfrak{g}$.

Definição A.2.48. ([18, p.640]) Seja $\mathfrak{g}$ uma álgebra de Lie de dimensão finita. Uma subálgebra h de $\mathfrak{g}$ é chamada de subálgebra de Cartan de $\mathfrak{g}$ se:

(a) h é nilpotente, $e$

(b) h é seu próprio normalizador em $\mathfrak{g}$.

Exemplo A.2.49. Ao considerarmos $\mathfrak{g}=\mathfrak{s l}(2)$ (sobre um corpo $\mathbb{K}$ de característica diferente de $2)$

$$
\mathfrak{h}=\left\{\left(\begin{array}{cc}
a & 0 \\
0 & -a
\end{array}\right), a \in \mathbb{K}\right\}
$$

é uma subálgebra de Cartan, pois $\mathfrak{h}$ é abeliana e, considerando

$$
\left\{X=\left(\begin{array}{ll}
0 & 1 \\
0 & 0
\end{array}\right), H=\left(\begin{array}{cc}
1 & 0 \\
0 & -1
\end{array}\right), Y=\left(\begin{array}{ll}
0 & 0 \\
1 & 0
\end{array}\right)\right\}
$$

temos $[H, \alpha X+\beta H+\gamma Y]=2 \alpha X-2 \gamma Y$, com $\alpha, \beta, \gamma \in \mathbb{K}$, que está em $\mathfrak{h}$ se e só se $\alpha=\gamma=0$, o que nos mostra que $\mathfrak{h}$ é igual ao seu normalizador.

Observação A.2.50. Conforme Proposição 4.26 em [4], o conjunto das matrizes diagonais em $\mathfrak{s l}(n, \mathbb{C})$ tem dimensão $n-1$ e é uma subálgebra de Cartan de $\mathfrak{s l}(n, \mathbb{C})$, para todo $n \in \mathbb{N}$. 


\section{A.3 Álgebras Envolventes Universais}

Ao estudarmos as representações da álgebra envolvente universal de uma álgebra de Lie $\mathfrak{g}$, obtemos também informações a respeito das representações de $\mathfrak{g}$.

Nesta seção, iremos enunciar os principais resultados para essas álgebras envolventes, baseandonos principalmente em [4] e [18]. Aqui, novamente, todos os espaços vetoriais serão sobre um corpo $\mathbb{K}$ arbitrário, salvo menção em contrário.

Destacamos ainda que, ao mencionarmos uma álgebra associativa, estaremos admitindo que ela possui elemento identidade.

Definição A.3.1. Seja $\mathfrak{g}$ uma álgebra de Lie. Por uma álgebra envolvente universal de $\mathfrak{g}$ iremos entender um par $(\mathfrak{U}, i)$ composto de uma álgebra associativa $\mathfrak{U}$ junto com uma aplicação $i: \mathfrak{g} \longrightarrow \mathfrak{U}$ satisfazendo as seguintes condições:

(a) A aplicação $i$ é um homomorfismo de álgebras de Lie de $\mathfrak{g}$ em $[\mathfrak{U}]$; isto é, i é linear, e para todo $x, y \in \mathfrak{g}, i([x, y])=i(x) i(y)-i(y) i(x)$;

(b) (Propriedade Universal)Dada qualquer álgebra associativa A e qualquer homomorfismo de álgebras de Lie $f: \mathfrak{g} \longrightarrow[A]$, existe um único homomorfismo de álgebras $f^{\prime}: \mathfrak{U} \longrightarrow A$ tal que o seguinte diagrama comuta:

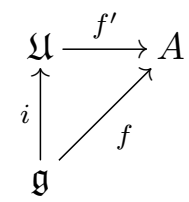

Em outras palavras, $f=f^{\prime} \circ i$.

Se uma álgebra de Lie $\mathfrak{g}$ possui álgebras envolventes universais $(\mathfrak{U}, i)$ e $\left(\mathfrak{U}^{\prime}, i^{\prime}\right)$, então $\mathfrak{U} \cong \mathfrak{U}^{\prime}$, no sentido de que existe um isomorfismo $f$ entre essas álgebras tal que $f \circ i=i^{\prime}$. Para a demonstração, que não faremos aqui, são utilizados os argumentos clássicos para objetos com propriedades universais; a existência é dada pela proposição seguinte.

Proposição A.3.2. Seja $\mathfrak{g}$ uma álgebra de Lie. Então, $\mathfrak{g}$ admite uma álgebra envolvente universal.

(vide [18, p.39-40])

Se estudarmos em detalhes a demonstração da Proposição A.3.2, veremos que a construção clássica da álgebra envolvente universal de $\mathfrak{g}$ é feita utilizando-se a álgebra tensorial $T$ do espaço vetorial $\mathfrak{g}$, e tomando-se o quociente dessa álgebra pelo ideal bilateral $\mathfrak{u}$ gerado por todos os elementos da forma $x \otimes y-y \otimes x-[x, y]$, com $x, y \in \mathfrak{g} ;$ considerando $\pi$ a aplicação canônica de $T$ em $T / \mathfrak{u}$, define-se $i$ como $\pi_{\Gamma_{T^{1}(\mathfrak{g})=\mathfrak{g}}}$. A partir disso, notemos que $\mathfrak{U}$ é gerada (como álgebra associativa) pelos elementos $i(x), x \in \mathfrak{g}$. A partir de agora, denotaremos a álgebra construída na Proposição A.3.2 por $\mathfrak{U}(\mathfrak{g})$.

Observação A.3.3. Se $\mathfrak{g}_{1}$ e $\mathfrak{g}_{2}$ são álgebras de Lie sobre um corpo $\mathbb{K}$ e $f: \mathfrak{g}_{1} \longrightarrow \mathfrak{g}_{2}$ é um 
homomorfismo, então existe um único homomorfismo $\mathfrak{U}(f): \mathfrak{U}\left(\mathfrak{g}_{1}\right) \longrightarrow \mathfrak{U}\left(\mathfrak{g}_{2}\right)$ tal que

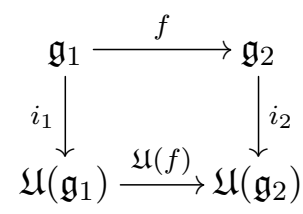

comuta.

De fato, como $f\left(\mathfrak{g}_{1}\right) \subseteq \mathfrak{g}_{2}$, então $i_{2} \circ f: g_{1} \longrightarrow \mathfrak{U}\left(\mathfrak{g}_{2}\right)$ é um homomorfismo de álgebras de Lie; pela propriedade universal, existe um único homomorfismo de álgebras $\mathfrak{U}(f): \mathfrak{U}\left(\mathfrak{g}_{1}\right) \longrightarrow \mathfrak{U}\left(\mathfrak{g}_{2}\right)$ tal que $\mathfrak{U}(f) \circ i_{1}=i_{2} \circ f$.

Observação A.3.4. Seja agora $\mathfrak{g}$ uma álgebra de Lie sobre um corpo $\mathbb{K}$, e $\pi: \mathfrak{g} \longrightarrow \mathfrak{g l}(V)$ uma representação de $\mathfrak{g}$ em um $\mathbb{K}$-espaço vetorial $V$. Segue diretamente da propriedade universal que $\pi$ estende-se a um homomorfismo de álgebras associativas (que também denotaremos por $\pi$ ) $\pi: \mathfrak{U}(\mathfrak{g}) \longrightarrow \mathfrak{g l}(V)$. Assim, dada uma representação de $\mathfrak{g}$ em $V$, temos também uma representação de $\mathfrak{U}(\mathfrak{g})$ em $V$.

Por outro lado, dada uma representação $\pi: \mathfrak{U}(\mathfrak{g}) \longrightarrow \mathfrak{g l}(V)$, de $\mathfrak{U}(\mathfrak{g})$ em um $\mathbb{K}$-espaço $V$, podemos "recuperar"uma representação de $\mathfrak{g}$ no mesmo espaço vetorial. Para isso, basta tomar $\pi \circ i: \mathfrak{g} \longrightarrow \mathfrak{g l}(V)$, e teremos um homomorfismo de álgebras de Lie, como desejado.

Teorema A.3.5 (Poincaré-Birkhoff-Witt(PBW)). Seja $\mathfrak{g}$ uma álgebra de Lie sobre um corpo $\mathbb{K}$, com uma base $\left\{x_{j} \mid j \in J\right\}$ indexada por algum conjunto $J$ totalmente ordenado. Seja (U, $i$ ) a álgebra envolvente universal de $\mathfrak{g}$. Então, a família de elementos

$$
i\left(x_{j_{1}}\right) i\left(x_{j_{2}}\right) \ldots i\left(x_{j_{n}}\right),
$$

com $j_{1} \leq j_{2} \leq \ldots \leq j_{n}, n \in \mathbb{Z}_{+}$, junto com $1 \in \mathbb{K}$ formam uma base do espaço $\mathfrak{U}$.

(vide [18, p.41-45])

Corolário A.3.6. As imagens $\left\{i\left(x_{j}\right)\right\}_{j \in J}$ em $\mathfrak{U}(\mathfrak{g})$ dos elementos da base $\left\{x_{j}\right\}_{j \in J}$ de $\mathfrak{g}$ formam um conjunto linearmente independente. Em particular,

$$
i: \mathfrak{g} \longrightarrow \mathfrak{U}(\mathfrak{g})
$$

é injetiva.

Assim, de agora em diante, podemos identificar $\mathfrak{g}$ como um subespaço de $\mathfrak{U}(\mathfrak{g})$. Se $x \in \mathfrak{g}$, escrevemos $x \in \mathfrak{U}(\mathfrak{g})$, ao invés de $i(x) \in \mathfrak{U}(\mathfrak{g})$.

Corolário A.3.7. Seja $\mathfrak{b}$ uma subálgebra da álgebra de Lie $\mathfrak{g}$. Suponha que $\left\{x_{j}\right\}_{j \in J} \cup\left\{y_{k}\right\}_{k \in K}$ seja uma base de $\mathfrak{g}$ tal que $\left\{x_{j}\right\}_{j \in J}$ é uma base de $\mathfrak{b}$ e tanto $J$ como $K$ são totalmente ordenados. Então,

(a) A injeção $\mathfrak{b} \longrightarrow \mathfrak{g} \longrightarrow \mathfrak{U}(\mathfrak{g})$ pode ser estendida a uma injeção de $\mathfrak{U}(\mathfrak{b})$ em $\mathfrak{U}(\mathfrak{g})$;

(b) $\mathfrak{U}(\mathfrak{g})$ é um $\mathfrak{U}(\mathfrak{b})$-módulo livre à esquerda (respectivamente à direita) admitindo a família $\left\{y_{k_{1}} \ldots y_{k_{n}} ; k_{1} \leq \ldots \leq k_{n}, n \in \mathbb{N}\right\}$ como uma base.

(vide $[18, \mathrm{p} .46]$ ) 
Corolário A.3.8. Sejam $\mathfrak{g}_{1}, \ldots, \mathfrak{g}_{n}$ álgebras de Lie, e defina $\mathfrak{g}=\mathfrak{g}_{1} \times \ldots \times \mathfrak{g}_{n}$ (produto direto de álgebras de Lie). Então,

$$
\mathfrak{U}(\mathfrak{g}) \cong \mathfrak{U}\left(\mathfrak{g}_{1}\right) \otimes \ldots \otimes \mathfrak{U}\left(\mathfrak{g}_{n}\right)
$$

como $\mathbb{K}$-espaços vetoriais.

Proposição A.3.9. Seja $\mathfrak{g}$ uma álgebra de Lie. A representação adjunta estende-se de forma única a uma representação (denotada por ad $d_{\mathfrak{u}}$ e também chamada de representação adjunta) de $\mathfrak{g}$ em $\mathfrak{U}(\mathfrak{g})$ pela qual $\mathfrak{g}$ age como uma derivação interna em $\mathfrak{U}(\mathfrak{g})$. Mais precisamente, para todo $x \in \mathfrak{g}$, $\mathfrak{u} \in \mathfrak{U}(\mathfrak{g})$

$$
a d_{\mathfrak{u}}(x)(u)=x u-u x
$$

(vide [18, p.47])

Proposição A.3.10. Seja $\mathfrak{g}$ uma álgebra de Lie. Então, $\mathfrak{U}(\mathfrak{g})$ não possui divisores de zero,isto é, para todo $a, b \in \mathfrak{U}(\mathfrak{g}), a b=0$ implica $a=0$ ou $b=0$.

(vide $[18$, p.48-49])

\section{A.4 Extensões Centrais}

Finalizando este apêndice, construiremos uma extensão central para uma álgebra de Lie que possui um 2-cociclo (extensão por um 2-cociclo); tal extensão será uma ferramenta importante no Capítulo 1, especificamente na construção de uma álgebra de Lie afim.

Definição A.4.1. Seja $\mathfrak{g}$ uma álgebra de Lie sobre um corpo $\mathbb{K}$. Um 2-cociclo a valores em $\mathbb{K}$ sobre $\mathfrak{g}$ é uma forma bilinear $\psi$ satisfazendo duas condições:

(a) $\psi(a, b)=-\psi(b, a)$;

(b) $\psi([a, b], c)+\psi([b, c], a)+\psi([c, a], b)=0$, para todo $a, b, c \in \mathfrak{g}$.

Definição A.4.2. Sejam $\mathfrak{g}$ e $\mathfrak{c}$ álgebras de Lie sobre um corpo $\mathbb{K}$. Por uma extensão central de $\mathfrak{g}$ por $\mathfrak{c}$ entenderemos uma sequência exata de álgebras de Lie

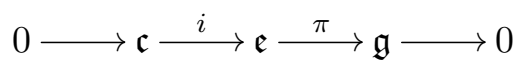

tal que $\mathfrak{c}$ está no centro de $\mathfrak{e}$. Em outras palavras, temos um homomorfismo sobrejetor de álgebras de Lie $\pi: \mathfrak{e} \longrightarrow \mathfrak{g}$ e um homomorfismo injetor $i: \mathfrak{c} \longrightarrow \mathfrak{e}$ tal que $\mathfrak{c} \cong$ Ker $\pi$ está no centro de $\mathfrak{e}$.

Com as definições acima, podemos efetuar a construção.

Sejam $\mathfrak{g}$ uma álgebra de Lie sobre um corpo $\mathbb{K}, \psi$ um 2-cociclo sobre $\mathfrak{g}$, e $\mathfrak{c}=\mathbb{K} v$ um espaço vetorial de dimensão 1 sobre $\mathbb{K}$. Considere $\mathfrak{e}=\mathfrak{c} \oplus \mathfrak{g}$ (soma direta de espaços vetoriais), e teremos uma estrutura de álgebra de Lie em $\mathfrak{e}$, com o colchete dado por 


$$
\left[\alpha v+g_{1}, \beta v+g_{2}\right]=\left[g_{1}, g_{2}\right]_{\mathfrak{g}}+\psi\left(g_{1}, g_{2}\right) v
$$

para todo $\alpha, \beta \in \mathbb{K}, g_{1}, g_{2} \in \mathfrak{g}$, e [, $]_{\mathfrak{g}}$ denotando o colchete de Lie em $\mathfrak{g}$. A estrutura de álgebra de Lie está garantida pelas propriedades de álgebra de Lie de $\mathfrak{g}$ e pelas propriedades de 2-cociclo de $\psi$. Notemos que, fazendo $\mathfrak{g}_{1}=0$, temos $(\alpha v, 0) \in \mathfrak{z}(\mathfrak{e})$, para todo $\alpha \in \mathbb{K}$.

Definindo então

$$
\begin{aligned}
i: \mathfrak{c} & \longrightarrow \mathfrak{e} \\
\alpha v & \longmapsto(\alpha v, 0) \mathrm{e} \\
\pi: \mathfrak{e} & \longrightarrow \mathfrak{g} \\
\left(\alpha v, g_{1}\right) & \longmapsto g_{1},
\end{aligned}
$$

temos $\operatorname{Im} i=\operatorname{Ker} \pi=\{(\alpha v, 0): \alpha \in \mathbb{K}\} \subseteq \mathfrak{z}(\mathfrak{e})$. Assim, temos uma extensão central de $\mathfrak{g}$ por $\mathfrak{c}$, chamada extensão por um 2-cociclo. 


\section{Referências Bibliográficas}

[1] M. F. Atiyah, I. G. Macdonald, Introduction to Commutative Algebra, Addison-Wesley Publishing Company, 1969.

[2] V. Bekkert, G. Benkart, V. Futorny, I. Kashuba, New irreducible modules for Heisenberg and affine Lie algebras, Journal of Algebra 373 (2013), 284-298.

[3] D. Bernard, G. Felder, Fock Representations and BRST Cohomology in SL(2) Current Algebra, Communications in Mathematical Physics 127 (1990), 145-168.

[4] R. Carter, Lie Algebras of Finite and Affine Type, Cambridge University Press, 2005.

[5] E. Frenkel, D. Ben-Zvi, Vertex Algebras and Algebraic Curves, Mathematical Surveys and Monographs, Volume 88, AMS, 2004.

[6] V. Futorny, Realizations of Affine Lie Algebras, Algebra and Discrete Mathematics 1 (2005), $30-46$.

[7] V. Futorny, D. Grantcharov, R. A. Martins, Localization of Free Field Realizations of Affine Lie Algebras, arXiv .

[8] V. M. Futorny, On imaginary Verma modules over the affine Lie algebra $A_{1}^{(1)}$, Oslo University 9 (1991), 213-218.

[9] V. M. Futorny, Imaginary Verma Modules for Affine Lie Algebras, Canadian Mathematical Bulletin 37 (1994), 213-218.

[10] K. R. Goodearl, J. R. B .Warfield, An Introduction to Noncommutative Noetherian Rings, Cambridge University Press, 2004.

[11] N. Jacobson, Basic Algebra II, W. H. Freeman and Company, 1989.

[12] H. P. Jakobsen, V. G. Kac, A New Class of Unitarizable Highest Weight Representations of Infinite Dimensional Lie Algebras, Lecture Notes in Physics 226 (1985), 1-20.

[13] V. Kac, Vertex Algebras for Beginners, University Lecture Series, Volume 10, AMS, 1998.

[14] V. G. Kac, Infinite Dimensional Lie Algebras, Cambridge University Press, 1990.

[15] L. A. B. S. Martin, Álgebras de Lie, Editora UNICAMP, 2010.

[16] R. A. Martins, Free field realizations of certain modules for affine Lie algebra $\hat{\mathfrak{s} l}(n, \mathbb{C})$, Algebra and Discrete Mathematics 12 (2011), 28-52.

[17] O. Mathieu, Classification of irreducible weight modules, Annales de l'institut Fourier 50 (2000), $537-592$.

[18] R. V. Moody, A. Pianzola, Lie Algebras With Triangular Decompositions, Wiley-Interscience, 1995. 
[19] J. J. Rotman, Advanced Modern Algebra, Prentice Hall, 2002.

[20] R. Y. Sharp, Steps in Commutative Algebra, Cambridge University Press, 2000.

[21] M. Wakimoto, Fock Representations of the Affine Lie Algebra $A_{1}^{(1)}$, Communications in Mathematical Physics 104 (1986), 605-609. 